\title{
Die aktienrechtliche Sanierung: 11. Tagung Sanierung und Insolvenz von Unternehmen Tagungsband 2020
}

Cavelti, Ladina ; Stössel, Jasmine ; Blanke, Ulf ; Zahnd, Bettina ; Wenk, Thomas ; Schimpel, Ulrich

DOI: https://doi.org/10.36862/eiz-364

Posted at the Zurich Open Repository and Archive, University of Zurich ZORA URL: https://doi.org/10.5167/uzh-203258

Edited Scientific Work

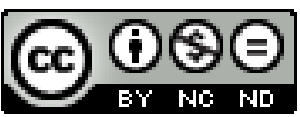

The following work is licensed under a Creative Commons: Attribution-NonCommercial-NoDerivatives 4.0 International (CC BY-NC-ND 4.0) License.

Originally published at:

Cavelti, Ladina; Stössel, Jasmine; Blanke, Ulf; Zahnd, Bettina; Wenk, Thomas; Schimpel, Ulrich Die aktienrechtliche Sanierung: 11. Tagung Sanierung und Insolvenz von Unternehmen Tagungsband 2020. Edited by: Sprecher, Thomas (2021). Zürich: EIZ Publishing.

DOI: https://doi.org/10.36862/eiz-364 


\section{+(4) \\ EuropaInstitut \\ AN DER UNIVERSITÄT ZÜRICH}

\section{Die aktienrechtliche Sanierung}

11. Tagung Sanierung und Insolvenz von Unternehmen Tagungsband 2020

EIZ $*_{*+}^{* *}$ (4) Publishing 



\section{(ii) \\ EuropaInstitut \\ AN DER UNIVERSITÄT ZÜRICH}

Herausgeber:

Thomas Sprecher

\section{Die aktienrechtliche Sanierung}

11. Fachtagung Sanierung und Insolvenz von Unternehmen Tagungsband 2020

EIZ $*_{* * *}^{* *}$ Publishing 


\section{(우 (1) $\circledast \odot$}

Die aktienrechtliche Sanierung von Thomas Sprecher wird unter Creative Commons

Namensnennung-Nicht kommerziell-Keine Bearbeitung 4.0 International lizenziert, sofern nichts anderes angegeben ist.

(C) 2021 - CC BY-NC-ND (Werk), CC BY-SA (Text)

Editors: Thomas Sprecher - Europa Institut an der Universität Zürich

Publishing \& production: buchundnetz.com

Cover: buch\&netz

ISBN:

978-3-03805-364-4 (Print - Softcover)

978-3-03805-424-5 (PDF)

978-3-03805-425-2 (ePub)

978-3-03805-426-9 (mobi/Kindle)

DOI: https://doi.org/10.36862/eiz-364

Version: 1.01-20210504

Dieses Werk ist als buch \& netz Online-Buch und als eBook in verschiedenen Formaten, sowie als gedrucktes Buch verfügbar. Weitere Informationen finden Sie unter der URL:

http://buchundnetz.com/werke/die-aktienrechtliche-sanierung/. 


\section{Vorwort}

Dieser Band versammelt, teilweise in erweiterter Form, die Referate der 11. Tagung „Sanierung und Insolvenz von Unternehmen“, die das Europa Institut an der Universität Zürich für den 9. Juni 2020 geplant hat. Pandemiebedingt konnte sie leider nicht physisch durchgeführt werden. Hingegen standen die Referate ab dem 9. Juni 2020 für eine gewisse Zeit online zur Verfügung.

Die Tagung schloss inhaltlich an jene des Vorjahrs an. Wiederum ging es um die aktienrechtliche Sanierung. Ein Schwergewicht liegt bei der Buchhaltung. Behandelt wird sodann mit dem Stillhalteabkommen eine weitere Form aktienrechtlicher Sanierungsmassnahmen. Die Tagung blickte sodann voraus: Beleuchtet werden auch die Sanierung nach neuem Aktienrecht sowie die Sanierung und Einlagensicherung nach neuem Bankenrecht.

Für ihre Beiträge und die Veröffentlichung in diesem Band danke ich den Referentinnen und Referenten sehr herzlich, wie auch Nathalie Kuhn für die Organisation der Videokonferenz und Noura Mourad, Sue Osterwalder und Petra Bitterli für die Gestaltung dieses Bandes.

Zürich, im März 2021

Thomas Sprecher 



\section{Inhaltsübersicht}

Sanierung von Unternehmen 9

Dr. iur. GIORGIO MEIER-MazzuCATO, dipl. Treuhandexperte, dipl.

Steuerexperte, Fachmann Finanz-und Rechnungswesen mit eidg. FA,

Revisionsexperte eidg. Revisionsaufsichtsbehörde, Partner bei ITERA

Corporate Finance, Bezirksrichter Zivil- und Strafgericht Aarau

Spurensuche - SchKG-Relevantes in der Buchhaltung

lic. iur. SIKANDER VON BHICKNAPAHARI, dipl. Experte in

Rechnungslegung und Controlling, zugelassener Revisionsexperte

Buchhalterische Darstellung von Sanierungsmassnahmen

\section{am praktischen Beispiel}

Dr. iur. OLIVER KÄLIN, Rechtsanwalt, LL.M., kaelin.legal AG, Zürich

Das Stillhalteabkommen - insolvenzrechtliche Aspekte

Dr. iur. MARC BERNHEIM, Rechtsanwalt, LL.M., Partner bei STAIGER

Rechtsanwälte AG, Zürich

lic. iur. GAUDEnZ GeIGeR, Rechtsanwalt, LL.M., Partner bei STAIGER

Rechtsanwälte AG, Zürich

Die Sanierung im neuen Aktienrecht

lic. iur. BRIGITTE KNECHT, Rechtsanwältin, LL.M., Counsel, Niederer

Kraft Frey AG, Zürich

MLaw LIVIA KELLER, Rechtsanwältin, LL.M., Niederer Kraft Frey AG,

Zürich

Sanierung und Einlagensicherung nach neuem Banken-

recht

Dr. iur. RETO SCHILTKNECHT, Rechtsanwalt, Leiter Internationales und Policy, Geschäftsbereich Recovery und Resolution, Eidgenössische Finanzmarktaufsicht FINMA, Bern, Lehrbeauftragter an der Universität St. Gallen 



\title{
Sanierung von Unternehmen
}

\author{
Giorgio Meier-Mazzucato*
}

\section{Inhalt}

I. Begriffe 10

1. Sanierung 10

2. Krise bzw. finanzielle Notlage 10

II. Begriff der Unterbilanz und der Überschuldung 11

1. Die buchhalterische Unterteilung der Unterbilanz 12

2. Die handelsrechtliche Unterteilung der Unterbilanz 14

III. Durchführung einer Sanierung 18

1. Sanierung als Problemlösungszyklus 18

2. Weitere Voraussetzungen einer erfolgreichen Sanierung 19

IV. Die Sanierungsarten und -formen bzw. -massnahmen 20

1. Die bilanzielle Sanierung 21

2. Die finanzielle Sanierung 27

3. Die organisatorische Sanierung 29

V. Überschuldungsprüfung und Sanierungsplan, Vorgehen der Revisionsstelle bei Überschuldung $\quad 30$

VI. Steuerfolgen der Sanierungsmassnahmen 31

1. Steuerfolgen aus Sicht des zu sanierenden Unternehmens 32

2. Steuerfolgen aus Sicht der Beteiligten 39

3. Sanierungsmassnahmen bei Tochter- und Schwestergesellschaften 43

4. Sanierungsfusion mit Tochter- und Schwestergesellschaften 45

Literaturverzeichnis $\quad 46$

\begin{tabular}{lr} 
Materialien & 49 \\
\hline
\end{tabular}

* Diese Arbeit gibt einen kurzen Überblick über Sanierungsgrundsätze hinsichtlich verschiedener Aspekte und zeigt einzelne Schritte und Möglichkeiten von Sanierungsmassnahmen.

Sie erhebt keineswegs Anspruch auf Vollständigkeit. Für weiterführende Darstellungen sei hiermit auf die entsprechende Literatur verwiesen.

Diese Arbeit ist, soweit sinnvoll, geschlechtsneutral formuliert. Um die Lesbarkeit nicht zu erschweren, werden Begriffe wie Übergeber, Übernehmer, Unternehmer usw. mehrheitlich in der männlichen Form geführt. Selbstverständlich und natürlich ist die jeweilige weibliche Form ebenfalls gemeint. 


\section{Begriffe}

\section{Sanierung}

\section{Sanierung}

Sanierung kommt aus dem Lateinischen und heisst zu Deutsch: Gesundung oder Heilung. Sanierung im Zusammenhang mit Unternehmungen bedeutet also die Gesundung oder Heilung von „kranken“ Unternehmungen.

Die Sanierung von Unternehmen kann in einem weiteren und engeren Sinn verstanden werden.

\section{Sanierung im weiteren und engeren Sinn}

- Sanierung im weiteren Sinn

Darunter werden alle bilanziellen, finanziellen und organisatorischen Massnahmen zur Wiederherstellung des durch Verluste angegriffenen Eigenkapitals einer Unternehmung verstanden.

- Sanierung im engeren Sinn

Darunter wird die Gesamtheit der finanziellen Massnahmen zur Wiederherstellung des finanziellen Gleichgewichts eines Unternehmens verstanden.

Unternehmenskrisen werden durch konjunkturelle, strukturelle oder führungsmässige Schwierigkeiten ausgelöst und bewirken folglich eine finanzielle Notlage.

\section{Krise bzw. finanzielle Notlage}

Wie äussert sich für ein Unternehmen eine Krise bzw. eine finanzielle Notlage? Sie äussert sich i.d.R. durch: 


\begin{tabular}{|l|l|}
\hline Ursachen führen zu & Wirkung der Äusserung der Ursachen \\
\hline Illiquidität & $\begin{array}{l}\text { Die zur Verfügung stehenden liquiden Mittel reichen nicht } \\
\text { mehr für eine fristgerechte Begleichung der Verbindlichke- } \\
\text { ten. } \\
\text { In der Finanz- und Betriebswirtschaft wird die Liquidität } \\
\text { als der „Atem“ eines Unternehmens bezeichnet. }\end{array}$ \\
\hline $\begin{array}{l}\text { Mangelnde } \\
\text { Rentabilität }\end{array}$ & $\begin{array}{l}\text { Die Rentabilität (Reingewinn im Verhältnis zum Kapital) ist } \\
\text { zu gering. Es wurden zu kleine Gewinne oder gar Verluste } \\
\text { erzielt. } \\
\text { In der Finanz- und Betriebswirtschaft wird die Rentabilität } \\
\text { als die „Nahrung“ eines Unternehmens bezeichnet. }\end{array}$ \\
\hline $\begin{array}{l}\text { Unterbilanz und } \\
\text { Überschuldung }\end{array}$ & $\begin{array}{l}\text { Bei der Unterbilanz ist das Eigenkapital z.T. verloren, d.h. } \\
\text { die Aktiven decken die Passiven nicht mehr voll. } \\
\text { Die Überschuldung zeigt sich dadurch, dass die Aktiven das } \\
\text { Fremdkapital nicht mehr voll decken. M.a.W. ist das Eigen- } \\
\text { kapital vollständig verloren. }\end{array}$ \\
\hline Falsche Finanzierung & $\begin{array}{l}\text { Das Fremd- und Eigenkapitalverhältnis entspricht nicht der } \\
\text { Aktivseite. Die goldene Bilanzregel und die goldene Finan- } \\
\text { zierungsregel sind nicht eingehalten. }\end{array}$ \\
\hline
\end{tabular}

\section{Begriff der Unterbilanz und der Überschuldung}

Unterbilanz kann als übergreifender Begriff verstanden werden, der sich weiter in eine Unterbilanz i.e.S., d.h. ohne Überschuldung, und eine Unterbilanz mit Überschuldung unterteilen lässt.

\section{Unterbilanz}

Umfassend (i.w.S.) kann die Unterbilanz umschrieben werden als die Situation, in der die Aktiven die Passiven nicht mehr decken.

Die Unterbilanz ist dann ohne Überschuldung, wenn

- der Bilanzverlust kleiner als das übrige Eigenkapital ist oder umgekehrt

- das Fremdkapital kleiner als die Aktiven, aber das Grundkapital nicht mehr voll gedeckt ist.

Die Unterbilanz ist eine Überschuldung, wenn

- der Bilanzverlust grösser als das Eigenkapital ist oder umgekehrt

- das Fremdkapital grösser als die Aktiven ist. 
Siehe in diesem Zusammenhang als aktienrechtliche Bestimmung zu Unterbilanz bzw. Kapitalverlust und Überschuldung Art. 725 Abs. 1 und 2 OR bzw. Art. 725a und 725b nOR, welche nebst für Aktiengesellschaften auch für $\mathrm{GmbH}$ und Genossenschaften gelten. Zu erwähnen ist, dass Art. 725 OR und Art. 725b ff. nOR auch unter dem neuen Rechnungslegungsrecht (Änderung vom 23. Dezember 2011 und in Kraft gesetzt auf 1. Januar 2013) bestehen bleiben. S. zur Bestimmung selbst und weiteren Ausführungen dazu unten Kapitel 3.2. Die handelsrechtliche Unterteilung der Unterbilanz.

\section{Die buchhalterische Unterteilung der Unterbilanz}

Die Unterteilung der Unterbilanz in

- offen

- verdeckt

- echt

- unecht

wird in der Folge anhand von einfachen Bilanzen bestimmt.

\section{Fall 1: Offener, echter Kapitalverlust}

\begin{tabular}{|c|c|c|c|}
\hline Aktiven & & & Passiven \\
\hline & Fr. & & Fr. \\
\hline Umlaufvermögen & $100 ’ 000$ & Fremdkapital & $1600^{\prime} 000$ \\
\hline \multirow[t]{3}{*}{ Anlagevermögen } & $70 ' 000$ & Grundkapital & $40 ' 000$ \\
\hline & & Bilanzverlust & $-30 \prime 000$ \\
\hline & $170 ’ 000$ & & $1700^{\prime} 000$ \\
\hline
\end{tabular}

Was für eine Unterbilanz zeigt sich hier und weshalb?

Es handelt sich um einen offenen, echten Kapitalverlust. Die Hälfte des Grundkapitals und der gesetzlichen Reserven sind nicht mehr gedeckt - nach Art. 725a Abs. 1 nOR sind es Aktienkapital, nicht an die Aktionäre zurückzahlbare gesetzliche Kapitalreserve und gesetzliche Gewinnreserve. Gemäss Art. 725 Abs. 1 OR bzw. Art. 725a Abs. 1 nOR müssen durch den Verwaltungsrat eine Generalversammlung einberufen und ihr Sanierungsmassnahmen beantragt werden.

Neu gemäss Art. 725a Abs. 2 nOR ist, dass in dem Fall, da die Gesellschaft keine Revisionsstelle hat, die letzte Jahresrechnung vor ihrer Genehmigung durch die Generalversammlung überdies einer eingeschränkten Revision durch einen zugelassenen Revisor unterzogen werden muss, wobei der Verwaltungsrat den zugelassenen Revisor ernennt. Diesbezüglich dürfte sich insofern eine 
gewisse Schwierigkeit zeigen, indem Revisoren tendenziell zu einer gewissen Vorsicht bei der Annahme eines Mandats zeigen, weshalb es u.U. nicht ganz einfach sein könnte, einen solchen zu finden und zu mandatieren. M.E. dürfte es im Hinblick auf die zunehmend statuierten Spezial- bzw. Auftragsrevisionen zu überlegen sein, ob eine Aktiengesellschaft ein allfälliges Opting out wieder aufgeben sollte.

Umgangen werden kann die soeben erwähnte Revisionspflicht, wenn der Verwaltungsrat ein Gesuch um Nachlassstundung einreicht (Art. 725a Abs. 3 nOR).

\section{Fall 2: Verdeckter, echter Kapitalverlust}

\begin{tabular}{lr|lr} 
Aktiven & \multicolumn{2}{c}{} & Passiven \\
\hline \multirow{3}{*}{ Umlaufvermögen } & Fr. & & Fr. \\
Anlagevermögen & 100 '000 & Fremdkapital & 150 '000 \\
& 90 '000 & Grundkapital & 40 '000 \\
& & Bilanzgewinn & 0 \\
\cline { 2 - 2 } & 190 '000 & & 190 '000 \\
\cline { 2 - 3 } & &
\end{tabular}

Das Anlagevermögen ist um Fr. 20 '000 über- und das Fremdkapital um Fr. 10'000 unterbewertet.

Was für eine Unterbilanz zeigt sich hier und weshalb?

Es handelt sich um einen verdeckten, aber echten Kapitalverlust von Fr. 30'000. Es kommt zur Anwendung von Art. 725 Abs. 1 OR bzw. Art. 725a nOR, da die Hälfte des Grundkapitals und der gesetzlichen Reserven nicht mehr gedeckt sind.

Fall 3: Kein handelsrechtlicher Kapitalverlust

\begin{tabular}{lr|lr} 
Aktiven & \multicolumn{2}{c}{ Passiven } \\
\hline & Fr. & & Fr. \\
Umlaufvermögen & 150 '000 & Fremdkapital & 150 '000 \\
Anlagevermögen & 110 '000 & Grundkapital & $100^{\prime} 000$ \\
& & Reserven & $50^{\prime} 000$ \\
& & Bilanzverlust & -40 '000 \\
& & & $260^{\prime} 000$ \\
\hline
\end{tabular}

Was für eine Unterbilanz zeigt sich hier und weshalb? 
Es handelt sich um keinen Kapitalverlust und der Bilanzverlust ist kleiner als die Hälfte des Grundkapitals und der gesetzlichen Reserven. Von Gesetzes wegen müssen daher keine Sanierungsmassnahmen vorgenommen werden.

\section{Fall 4: Offener, unechter Kapitalverlust}

\begin{tabular}{|c|c|c|c|}
\hline Aktiven & & & Passiven \\
\hline & Fr. & & Fr. \\
\hline Umlaufvermögen & $150 ’ 000$ & Fremdkapital & $1700^{\prime} 000$ \\
\hline \multirow[t]{4}{*}{ Anlagevermögen } & $90 \prime 000$ & Grundkapital & $100 ' 000$ \\
\hline & & Reserven & $50 \prime 000$ \\
\hline & & Bilanzverlust & $-80 ’ 000$ \\
\hline & $240 ' 000$ & & $240 ’ 000$ \\
\hline
\end{tabular}

Das Anlagevermögen ist um Fr. 20'000 unter- und das Fremdkapital um Fr. 20'000 überbewertet.

Was für eine Unterbilanz zeigt sich hier und weshalb?

Es handelt sich um einen offenen, unechten Kapitalverlust. Effektiv besteht ein Bilanzverlust von Fr. 40'000. Der effektive Bilanzverlust ist jedoch kleiner als die Hälfte des Aktienkapitals und der gesetzlichen Reserven. Von Gesetzes wegen müssen keine Sanierungsmassnahmen vorgenommen werden.

\section{Die handelsrechtliche Unterteilung der Unterbilanz}

Handelsrechtliche Bestimmungen zur Unterbilanz finden sich nur bei den Kapitalgesellschaften und der Genossenschaft. Die Personengesellschaften kennen keine entsprechenden Normen, da bei ihnen die Inhaber bzw. Gesellschafter, mit Ausnahme der Kommanditäre, für Schulden der Personenunternehmen persönlich und unbeschränkt haften. S. dazu Art. 544 Abs. 3 OR für die einfache Gesellschaft, Art. 569 OR für die Kollektivgesellschaft und Art. 604 und 612 OR für die Kommanditgesellschaft.

In der Folge werden stellvertretend für alle Kapitalgesellschaften und die Genossenschaft die Bestimmungen des Aktienrechts für die Unterbilanz betrachtet. Wesentlich sind demgemäss die Art. 670, 671 Abs. 3, 725 und 725a OR, ab 2013 960b OR bzw. nach neuem Aktienrecht Art. 725, 725a ff. nOR:

Gemäss Art. 670 OR bzw. Art. 725c nOR besteht die Möglichkeit der Aufwertung bestimmter Bilanzpositionen bei Kapitalverlust.

Art. 725 OR bzw. Art. 725a und 725b nOR geben dem Verwaltungsrat Handlungsanweisungen bei Kapitalverlust und Überschuldung. 
Im Zusammenhang mit der Überschuldung gemäss Art. 725b Abs. 2 nOR zeigt sich die analoge Problematik wie beim Kapitalverlust. S. dazu bereits oben die Hinweise zu Fall 1. Weiter verschärft sich die Situation, indem dem ernannten zugelassenen Revisor gemäss Art. 725b Abs. 5 nOR auch gleich die Anzeigepflichten der eingeschränkt prüfenden Revisionsstelle obliegen. Dass eine Mandatierung entsprechend schwierig sein dürfte ist evident und es verbleibt m.E. einmal mehr darauf hinzuweisen, Gesellschaften mit Opting out dieses aufzugeben und eine Revisionsstelle zu wählen.

Der bisherige Art. 725a OR zur Eröffnung oder dem Aufschub des Konkurses entfällt indessen. Der Vollständigkeit halber wird er aber aufgeführt, solange er in Kraft ist.

Von erheblicher Bedeutung im Zusammenhang mit Aufwertungen ausserhalb von Art. 670 OR bzw. Art. 725c nOR ist die Möglichkeit gemäss Art. 960b OR, Aktiven mit Börsenkursen oder beobachtbaren Marktpreisen zu diesen Werten am Bilanzstichtag zu erfassen.

In der Folge werden einige Beispiele von handelsrechtlichen Bilanzen gezeigt, um die vorstehenden Ausführungen zu veranschaulichen.

\section{Fall 1: Offener, echter Kapitalverlust}

\begin{tabular}{lr|lr} 
Aktiven & \multicolumn{2}{r}{} & Passiven \\
\hline \multirow{3}{*}{ Umlaufvermögen } & Fr. & & Fr. \\
Anlagevermögen & $150^{\prime} 000$ & Fremdkapital & $190^{\prime} 000$ \\
& $110^{\prime} 000$ & Aktienkapital & $100^{\prime} 000$ \\
& & Reserven & $50^{\prime} 000$ \\
& & Bilanzverlust & $-80^{\prime} 000$ \\
& & & 260 '000 \\
\hline
\end{tabular}

Hat diese Unterbilanz gesetzliche Folgen? Wie können die Reserven verwendet werden?

Es handelt sich um einen offenen, echten Kapitalverlust. Der Bilanzverlust ist grösser als die Hälfte des Aktienkapitals und der gesetzlichen Reserven und es müssten Massnahmen gemäss Art. 725 Abs. 1 OR ergriffen werden. S. Art. 725a Abs. 1 nOR, wonach die Hälfte der Summe aus Aktienkapital, nicht an die Aktionäre zurückzahlbarer gesetzlicher Kapitalreserve und gesetzlicher Gewinnreserve die Aktiven abzüglich der Verbindlichkeiten decken müssen. Gemäss Art. 671 Abs. 3 OR darf die allgemeine Reserve, soweit sie die Hälfte des Aktienkapitals nicht übersteigt, zur Deckung von Verlusten oder für Massnahmen verwendet werden, die geeignet sind, in Zeiten schlechten Geschäftsgan- 
ges das Unternehmen durchzuhalten, der Arbeitslosigkeit entgegenzuwirken oder ihre Folgen zu mildern. Die analoge Bestimmung nach neuem Aktienrecht ist Art. 672 Abs. 2 nOR, wonach die gesetzliche Gewinnreserve zu äufnen ist, bis sie zusammen mit der gesetzlichen Kapitalreserve die Hälfte des im Handelsregister eingetragenen Aktienkapitals erreicht.

Nach der Verrechnung der Reserven mit dem Bilanzverlust verbleibt ein solcher von Fr. 30'000, sodass der Kapitalverlust eliminiert ist und keine Sanierungsmassnahmen eingeleitet werden müssen.

\section{Fall 2: Offener, echter Kapitalverlust}

\begin{tabular}{lr|lr} 
Aktiven & \multicolumn{2}{c}{ Passiven } \\
\hline & Fr. & & Fr. \\
Umlaufvermögen & 90 '000 & Fremdkapital & 150 '000 \\
Anlagevermögen & 100 '000 & Aktienkapital & 100 '000 \\
& & Reserven & 50 '000 \\
& & Bilanzverlust & -110 '000 \\
\cline { 2 - 2 } & & & 190 '000 \\
\cline { 2 - 3 } & & & \\
\cline { 2 - 3 } & 190 '000 & &
\end{tabular}

Hat diese Unterbilanz gesetzliche Folgen? Wie können die Reserven verwendet werden?

Es handelt sich um einen offenen, echten Kapitalverlust. Der Bilanzverlust ist grösser als die Hälfte des Aktienkapitals und der gesetzlichen Reserven und es müssten Massnahmen gemäss Art. 725 Abs. 1 OR ergriffen werden. S. Art. 725a Abs. 1 nOR, wonach die Hälfte der Summe aus Aktienkapital, nicht an die Aktionäre zurückzahlbarer gesetzlicher Kapitalreserve und gesetzlicher Gewinnreserve die Aktiven abzüglich der Verbindlichkeiten decken müssen. Gemäss Art. 671 Abs. 3 OR darf die allgemeine Reserve, soweit sie die Hälfte des Aktienkapitals nicht übersteigt, zur Deckung von Verlusten oder für Massnahmen verwendet werden, die geeignet sind, in Zeiten schlechten Geschäftsganges das Unternehmen durchzuhalten, der Arbeitslosigkeit entgegenzuwirken oder ihre Folgen zu mildern. Die analoge Bestimmung nach neuem Aktienrecht ist Art. 672 Abs. 2 nOR, wonach die gesetzliche Gewinnreserve zu äufnen ist, bis sie zusammen mit der gesetzlichen Kapitalreserve die Hälfte des im Handelsregister eingetragenen Aktienkapitals erreicht. Die gesetzliche Kapitalreserve darf nach Art. 671 Abs. 2 nOR an die Aktionäre zurückbezahlt werden, wenn die gesetzlichen Kapital- und Gewinnreserven, abzüglich des Betrags allfälliger Verluste, die Hälfte des im Handelsregister eingetragenen Aktienkapitals übersteigen. 
Auch nach Verrechnung der Reserven mit dem Bilanzverlust verbleibt ein Kapitalverlust gemäss Art. 725 Abs. 1 OR von CHF 60'000 und es müssen entsprechende Sanierungsmassnahmen ergriffen werden.

\section{Fall 3: Offene Überschuldung}

\begin{tabular}{lr|lr} 
Aktiven & \multicolumn{2}{c}{ Passiven } \\
\hline \multirow{3}{*}{ Umlaufvermögen } & Fr. & & Fr. \\
Anlagevermögen & 90 '000 & Fremdkapital & $220^{\prime} 000$ \\
& 100 '000 & Aktienkapital & $100^{\prime} 000$ \\
& & Reserven & $50^{\prime} 000$ \\
& & Bilanzverlust & $-180^{\prime} 000$ \\
\cline { 2 - 2 } & & & $190^{\prime} 000$ \\
\hline
\end{tabular}

Hat diese Unterbilanz gesetzliche Folgen? Wie können die Reserven verwendet werden?

Es handelt sich um eine Überschuldung. Der Bilanzverlust ist grösser als das Aktienkapital und die gesetzlichen Reserven oder umgekehrt das Fremdkapital grösser als die Aktiven. Gemäss Art. 725 Abs. 2 OR bzw. Art. 725b nOR muss ein Zwischenabschluss zu Fortführungs- und Veräusserungswerten erstellt und diese einem zugelassenen Revisor zur Prüfung vorgelegt werden.

Die Verwendung der allgemeinen Reserve gemäss Art. 671 Abs. 3 OR bzw. Art. 671 Abs. 2 i.V.m. Art. 672 Abs. 3 nOR hat hier keine positiven Auswirkungen, da sie die Überschuldung nicht aufhebt.

Ist die Gesellschaft gemäss den beiden Zwischenabschlüssen überschuldet, so benachrichtigt der Verwaltungsrat gestützt auf Art. 725b Abs. 3 nOR das Gericht. Dieses eröffnet den Konkurs oder verfährt nach Art. 173a SchKG. Vorbehalten bleiben die Möglichkeiten gemäss Art. 725b Abs. 4 nOR.

Die nachfolgende Darstellung zeigt die Mathematik des Kapitalverlusts bzw. der Überschuldung, auch unter Berücksichtigung des Covid-19-Kredits gemäss Art. i.V.m. Art. 325 Covid-19-SBüG. 


\begin{tabular}{|c|c|c|c|c|}
\hline Kapitalverlust & $\begin{array}{r}\text { Ausgangslage } \\
\text { TFr. }\end{array}$ & $\%$ & $\begin{array}{r}\text { bilanziell } \\
\text { TFr. }\end{array}$ & $\begin{array}{r}\text { saniert } \\
\%\end{array}$ \\
\hline Grundkapital & 100 & 58.82 & 100 & 43.48 \\
\hline Kapitalreserven & 20 & 11.76 & 0 & 0.00 \\
\hline Gewinnreserven & 50 & 29.41 & 0 & 0.00 \\
\hline Covid-19-Kredit & 0 & 0.00 & 130 & 56.52 \\
\hline Zwischentotal & 170 & 100.00 & 230 & 100.00 \\
\hline Bilanzverlust & -100 & -58.82 & -30 & -13.04 \\
\hline Total Eigenkapital & 70 & 41.18 & 200 & 70.00 \\
\hline Überschuldung & $\begin{array}{r}\text { Ausgangslage } \\
\text { TFr. }\end{array}$ & $\%$ & $\begin{array}{r}\text { bilanziell } \\
\text { TFr. }\end{array}$ & $\begin{array}{r}\text { saniert } \\
\%\end{array}$ \\
\hline Grundkapital & 100 & 58.82 & 100 & 43.48 \\
\hline Kapitalreserven & 20 & 11.76 & 0 & 0.00 \\
\hline Gewinnreserven & 50 & 29.41 & 0 & 0.00 \\
\hline Covid-19-Kredit & 0 & 0.00 & 130 & 56.52 \\
\hline Zwischentotal & 170 & 100.00 & 230 & 100.00 \\
\hline Bilanzverlust & -190 & -111.76 & -120 & -52.17 \\
\hline Total Eigenkapital & -20 & -11.76 & 110 & 47.83 \\
\hline
\end{tabular}

\section{Durchführung einer Sanierung}

\section{Sanierung als Problemlösungszyklus}

Eine erfolgreiche Sanierung setzt voraus, dass die Vorgehensweise klar und strukturiert erfolgt. Aufgrund der Vielschichtigkeit und verschiedenen Abhängigkeiten empfiehlt es sich, die Sanierung als Problemlösungszyklus zu organisieren. S. dazu die folgende Darstellung. 


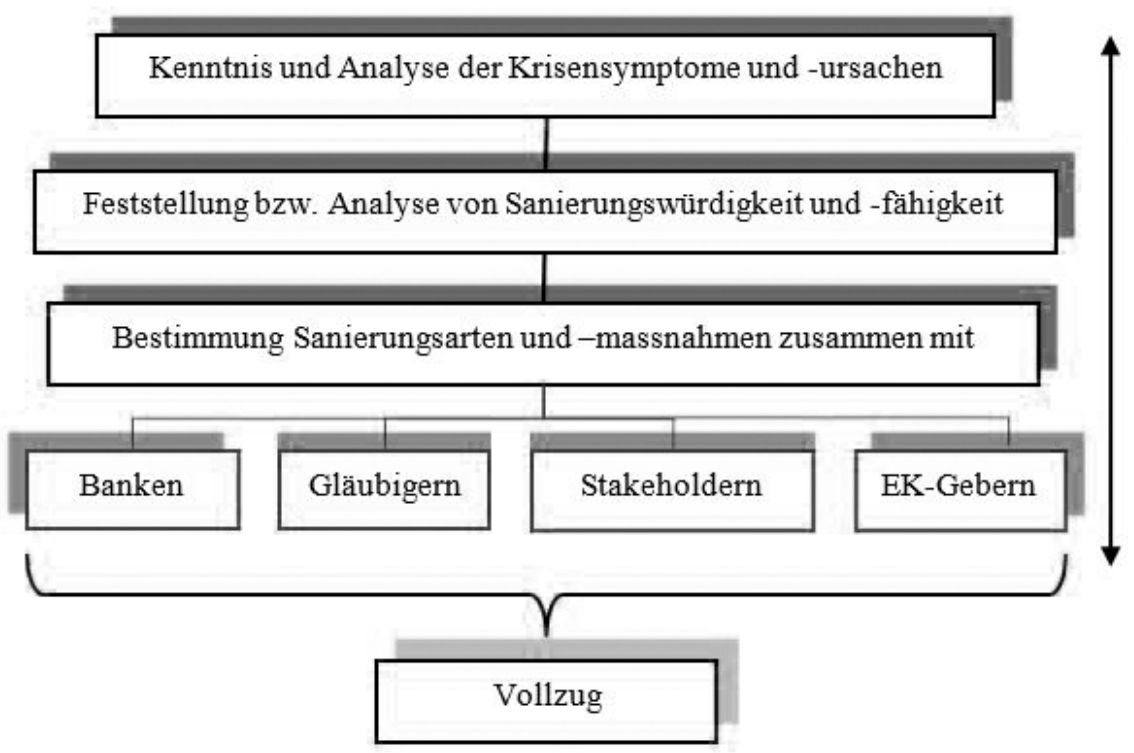

2. Weitere Voraussetzungen einer erfolgreichen Sanierung

Nebst Kenntnis der Krisenursachen ist eine grundlegende Voraussetzung für eine erfolgreiche Sanierung, dass Sanierungswürdigkeit und Sanierungsfähigkeit beim krisengebeutelten Unternehmen gegeben sind. S. dazu die folgende Darstellung.

\section{Voraussetzung erfolgreiche Sanierung}

\section{Sanierungswürdigkeit:}

Unternehmen noch zu retten

keine totale Illiquidität

keine totale Überschuldung

keine totale Betriebsunfähigkeit

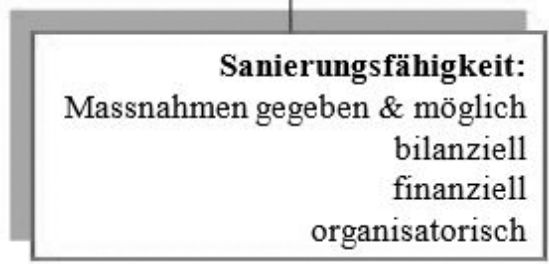




\section{Die Sanierungsarten und -formen bzw. -massnahmen}

Die Sanierungsmassnahmen i.w.S. lassen sich strukturell unterteilen in Sanierungsarten und Sanierungsformen mit folgenden Inhalten.

\section{Voraussetzung erfolgreiche Sanierung}

\section{Sanierungswürdigkeit:}

Unternehmen noch zu retten

keine totale Illiquidität

keine totale Überschuldung

keine totale Betriebsunfähigkeit

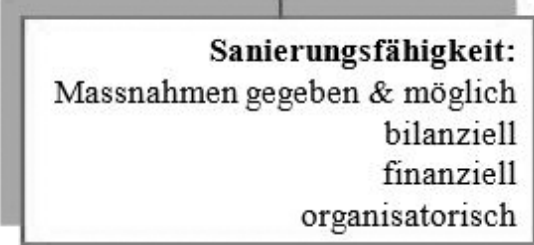

Die Sanierungsmassnahmen i.w.S. können in bilanzielle, finanzielle und organisatorische Sanierung unterteilt und mit Sanierungsformen bzw. -massnahmen kombiniert werden.

\begin{tabular}{|l|l|}
\hline Sanierungsarten & Sanierungsformen bzw. -massnahmen \\
\hline bilanzielle Sanierung & $\begin{array}{l}\text { - Kapitalherabsetzung } \\
- \text { Aufwertung von Aktiven } \\
- \text { Auflösung von stillen Reserven } \\
- \text { Rangrücktritt }\end{array}$ \\
\hline finanzielle Sanierung & $\begin{array}{l}- \text { Veränderung des Eigenkapitals } \\
- \text { Veränderung des Fremdkapitals }\end{array}$ \\
\hline $\begin{array}{l}\text { organisatorische San- } \\
\text { ierung }\end{array}$ & $\begin{array}{l}- \text { Personalstruktur } \\
- \text { Betriebsorganisation } \\
- \text { Finanz- und Rechnungswesen }\end{array}$ \\
\hline
\end{tabular}

Die einzelnen Sanierungsarten und -formen bzw. -massnahmen werden in der Folge im Detail kurz dargestellt und gleichzeitig die entsprechenden Buchungssätze gebildet. 


\section{Die bilanzielle Sanierung}

Die bilanzielle Sanierung als eine der drei Sanierungsarten wird vielfach wegen des damit verbundenen geringen Aufwands und ihrer schnellen Wirkung als erste Massnahme ergriffen.

\section{Bilanzielle Sanierung}

Die Charakteristik der bilanziellen Sanierung liegt darin, dass keine finanziellen Vorgänge notwendig sind.

Es sind nebst den für die Durchführung teilweise notwendigen organisatorischen Vorkehrungen lediglich entsprechende Buchungen zu treffen.

Nachfolgend werden die Formen und Massnahmen der bilanziellen Sanierung stichwortartig aufgeführt.

\section{Kapitalherabsetzung (Art. 732 - 735 OR bzw. Art. 653j - 653p nOR)}

a) Generalversammlungsbeschluss mit Änderung der Statuten (Art. 732 Abs. 1 OR bzw. Art. 653j und 653o nOR);

b) Revisionsbericht von zugelassenem Revisionsexperten (Art. 732 Abs. 2 OR bzw. Art. 653m OR);

c) Mindestaktienkapital Fr. 100’000 (Art. 732 Abs. 5 OR bzw. Art. 653j Abs. 3 nOR);

d) Nennwert pro Aktie bis auf 1 Rappen möglich (Art. 622 Abs. 4 OR) bzw. Nennwert grösser als null (Art. 622 Abs. 4 nOR);

e) Aufforderung, Befriedigung und Sicherstellung der Gläubiger (Art. 733 OR) nicht notwendig, wenn Kapitalherabsetzung kleiner als die Unterbilanz. Wird das Aktienkapital gemäss Art. 653p nOR zur teilweisen oder vollständigen Beseitigung einer durch Verluste entstandenen Unterbilanz herabgesetzt und bestätigt ein zugelassener Revisionsexperte zuhanden der Generalversammlung, dass der Betrag der Kapitalherabsetzung den Betrag dieser Unterbilanz nicht übersteigt, so finden die Bestimmungen der ordentlichen Kapitalherabsetzung zur Sicherstellung von Forderungen, zum Zwischenabschluss, zur Prüfungsbestätigung und zu den Feststellungen des Verwaltungsrats keine Anwendung.

Beispielbuchungen:

\begin{tabular}{|llll|l|}
\hline 2800 & Grundkapital & an 2970 & Bilanzverlust & 80000 \\
\hline 2800 & Grundkapital & an 2950 & Reserven & 20 '000 \\
\hline
\end{tabular}


$\mathrm{Zu}$ erwähnen ist, dass die Buchungen allgemein über Sanierungskonto vorgenommen werden und hier aus Platzgründen direkt erfolgen. S. zum Sanierungskonto unten Kapitel 6. Die Sanierungsbilanz und das Sanierungskonto.

\section{Aufwertung bestimmter Aktiven (Art. 670 OR bzw. Art. 725c nOR)}

Die bestehende Bestimmung zur besagten Aufwertung gemäss Art. $670 \mathrm{OR}$ spricht lediglich von einem Kapitalverlust. Die analoge Anordnung gemäss Art. 725c nOR erfasst jedoch einen Kapitalverlust nach Art. 725a nOR als auch eine Überschuldung laut Art. 725b nOR. Da der Kapitalverlust die kleinere Beschädigung des Eigenkapitals ist als die Überschuldung, ist auch bereits unter dem aktuellen Recht eine Überschuldung sinngemäss miteingeschlossen.

Folgende Kriterien sind zu beachten:

a) Kapitalverlust (Art. 725 Abs. 1 OR, Art. 725a nOR) bzw. Überschuldung (Art. 725 Abs. 2 OR, Art. 725b nOR);

b) Aufwertung von Grundstücken oder Beteiligungen (Art. 670 Abs. 1 OR, Art. 725c Abs. 1 nOR);

c) Bildung der Aufwertungsreserve (Art. 671b OR, Art. 725c Abs. $1 \mathrm{nOR}$ );

d) Revisionsbericht der Revisionsstelle bzw. eines zugelassenen Revisors (Art. 670 Abs. 2 OR, Art. 725 Abs. 2 nOR);

e) Auflösung Aufwertungsreserve durch Umwandlung in Aktien- oder Partizipationskapital bzw. durch Wertberichtigung oder Veräusserung der aufgewerteten Aktiven (Art. 725c Abs. 3 nOR).

Das neue Aktienrecht äussert sich hinsichtlich der Prüfung der Aufwertung durch einen zugelassenen Revisor zur Situation, da eine Aktiengesellschaft das Opting out vorgenommen hat, mithin über keine Revisionsstelle mehr verfügt. In diesem Fall bestimmt das neue Aktienrecht in Art. 725c Abs. 3 nOR, dass ein zugelassener Revisor die Prüfung und Bestätigung vornimmt. Die betroffene Aktiengesellschaft ohne Revisionsstelle muss folglich, falls sie eine Aufwertung vornehmen möchte, zuerst einen zugelassenen Revisor finden und beauftragen. Gerade für den Fall einer Überschuldung könnte dies eine Schwierigkeit darstellen.

Aufwertung Beteiligungen und Liegenschaften $\rightarrow$ Art. 670 OR bzw. Art. 725c nOR 
- Bewertungsgrundsätze nach Rechnungslegungsrecht $\rightarrow 960$ Abs. 1 OR

Aktiven und Verbindlichkeiten werden i.d.R. einzeln bewertet, sofern sie wesentlich sind und aufgrund ihrer Gleichartigkeit für Bewertung nicht üblicherweise als Gruppe zusammengefasst werden.

S. dazu HWP 2014, S. 60 ff., insbesondere hinsichtlich Zwangsreserven und bspw. für Beteiligungen, HWP 2014, S. 175 ff.

- Gruppenbewertung Beteiligungen $\rightarrow$ HWP 2014, S. 177

Von Grundsatz Einzelbewertung kann nur in sachlich begründeten Einzelfällen abgewichen werden. So kann es vertretbar sein, verschiedene Beteiligungen, die bereits unternehmensintern in Führung und Beurteilung als wirtschaftliche Einheit betrachtet werden, als Bewertungseinheit zusammenzufassen.

Beispiel: Es kann gerechtfertigt sein, die Abwertung einer Beteiligung an einer ertragsschwachen Vertriebsgesellschaft mit dem Hinweis zu unterlassen, dass sich die ungenügenden Erträge aus betrieblichen Gründen ergeben und die beliefernde Produktionsgesellschaft entsprechende Gewinne erzielt.

Grundsätze der Bewertung von Beteiligungen

- S. nebst der Fachmitteilung Unternehmensbewertung 2018 auch Kommentar von Hüttche/Meier-Mazzucato, Kommentierung der Fachmitteilung Unternehmensbewertung, 1. Auflage 2018, S. 28:

... Auch hier kann auf die obigen Konkretisierungen - Marktpreis, gutachterlich approximierter Marktwert oder Vergleichswert - zurückgegriffen werden. Eine gutachterliche Lösung muss auf einer „stand alone“-Basis erfolgen. Entsprechend ist nur auf die tatsächlich übertragbare Ertragskraft und unechte Synergien - also von jedem Erwerber realisierbare Kombinationseffekte - abzustellen. Die Ausstattung der Anteile und die Beteiligungshöhe sind zu berücksichtigen.

- Ergo sind folgende relevante Aspekte zu beachten

- Wirklicher Wert $=$ innerer Wert $=$ gutachterlich objektivierter Wert. S. auch Kommentar Hüttche/Meier-Mazzucato, S. 23.

- Stand Alone = Frage der Übertragbarkeit der Ertragskraft. S. dazu Fachmitteilung Unternehmensbewertung 2018, Kapitel 3.3.2.4. Dabei zeigen sich folgende Berechnungsvarianten und -modelle; s. dazu Kommentar von Hüttche/Meier-Mazzucato, Kommentierung der Fachmitteilung Unternehmensbewertung, 1. Auflage 2018, S. 86: 


\begin{tabular}{|c|c|c|c|}
\hline \multicolumn{4}{|c|}{ Übertragbarkeit der Ertragskraft } \\
\hline $\begin{array}{c}\text { voll } \\
\text { übertragbar }\end{array}$ & $\begin{array}{l}\text { eingeschränkt } \\
\text { übertragbar }\end{array}$ & $\begin{array}{l}\text { temporär } \\
\text { übertragbar }\end{array}$ & $\begin{array}{c}\text { nicht } \\
\text { übertragbar }\end{array}$ \\
\hline $\begin{array}{c}\text { DCF- } \\
\text { Standardmodell }\end{array}$ & $\begin{array}{l}\text { Anpassung sofort } \\
\text { wegfallende } \\
\text { Faktoren }\end{array}$ & $\begin{array}{l}\text { Abschmelzen } \\
\text { Übergewinne }\end{array}$ & $\begin{array}{c}\text { Make or Buy } \\
\text { als } \\
\text { Varianten }\end{array}$ \\
\hline
\end{tabular}

- Nur unechte Synergien

- Ausstattung Anteile und Beteiligungshöhe $\rightarrow$ Paketzu- bzw. -abschläge. S. dazu Meier-Mazzucato/Montandon, Wert- bzw. Preisperspektiven und Paketzu- und -abschläge bei der Unternehmensbewertung und bei Unternehmenstransaktionen, TREX 52011

- Bei KMU kommt Einfluss Eigentümer besondere Bedeutung zu und damit auch der Frage der Übertragbarkeit der Ertragskraft des Unternehmens, nachdem diese bei KMU häufig personenbezogen ist. Sie kann demnach

- voll $\rightarrow$ DCF-Standardmodell,

- nur teilweise $\rightarrow$ Elimination wegfallender Faktoren,

- nur temporär $\rightarrow$ Abschmelzen Übergewinne bzw. temporäre Übergewinnkapitalisierung,

- oder nicht $\rightarrow$ Substanzwert mit make or buy

übertragbar sein.

Für die beschränkte Übertragbarkeit der Ertragskraft zu ermitteln bzw. zu berechnen können bspw. die beiden folgenden Modelle eingesetzt werden:

- Abschmelzen der Übergewinne $\rightarrow$ dazu Fachmitteilung Unternehmensbewertung 2018, Ziff. 77 ff. und Hüttche/Meier-Mazzucato, S. 85 ff.;

- Economic Value Added-Methode (EVA) mit temporärer Übergewinnkapitalisierung.

Beispielbuchungen für Bildung der Aufwertungsreserve:

\begin{tabular}{|c|c|c|c|c|c|}
\hline 60 & Grundstücke & an & 2940 & Aufwertungsreserve & $50 \prime 000$ \\
\hline 1480 & Beteiligungen & an & 2940 & Aufwertungsreserve & $50{ }^{\prime} 0$ \\
\hline
\end{tabular}

Beispielbuchungen für Auflösung der Aufwertungsreserve:

\begin{tabular}{|llrll|r|}
\hline 2940 & Aufwertungsreserve & an 1600 & Grundstücke & $50^{\prime} 000$ \\
\hline 2940 & Aufwertungsreserve & an 1480 & Beteiligungen & 50 '000 \\
\hline \multicolumn{7}{r}{} & oder & & \\
\hline 2940 & Aufwertungsreserve & an 2800 & Grundkapital & $100^{\prime} 000$ \\
\hline
\end{tabular}




\section{Aufwertung Aktiven mit beobachtbaren Marktpreisen (Art. 960b OR)}

In der Folgebewertung dürfen Aktiven mit Börsenkurs oder einem anderen beobachtbaren Marktpreis in einem aktiven Markt zum Kurs oder Marktpreis am Bilanzstichtag bewertet werden, auch wenn dieser über dem Nennwert oder dem Anschaffungswert liegt. Wer von diesem Recht Gebrauch macht, muss alle Aktiven der entsprechenden Positionen der Bilanz, die einen beobachtbaren Marktpreis aufweisen, zum Kurs oder Marktpreis am Bilanzstichtag bewerten. Im Anhang muss auf diese Bewertung hingewiesen werden. Es handelt sich bspw. um:

a) Vorräte

b) Wertschriften in den Finanzanlagen

* $\quad$ Grundstücke und Beteiligungen grundsätzlich nicht, da sie nicht als Aktiven mit beobachtbaren Marktpreisen gelten. Dies wäre lediglich dann zu bejahen, wenn sie sich in einem intakten Markt mit vergleichbaren Objekten befinden, bspw. von kotierten Beteiligungen

In Übereinstimmung mit Art. 670 OR müssen für Aufwertungen, welche nicht Grundstücke oder Beteiligungen betreffen, keine Aufwertungsreserven gebildet werden. Die Verbuchung solcher Positionen kann mithin direkt erfolgen.

\section{Auflösung von stillen Reserven}

a) auf Aktiven bis zum Anschaffungs- oder Herstellungskostenwert, ggf. auch bis zum Marktpreis, falls Aktiven mit beobachtbaren Marktpreisen (Art. 960a Abs. 1 und 2 und 960b OR);

b) auf Fremdkapital, insbesondere Rückstellungen (Art. 960a Abs. 4 und Art. 960e Abs. 4 OR).

S. zu den stillen Reserven die beiden nachfolgenden Übersichten: 
Übersicht 1: Bewertungsdifferenzen zwischen Rechnungslegungs- und Bilanzsteuerrecht

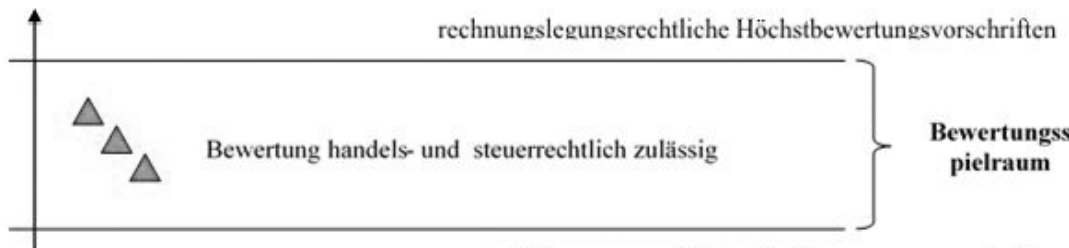

bilanzsteuerrechtliche Tiefstbewertungsvorschriften

steuerliche Aufrechnung/en

Übersicht 2: Arten und Entstehungsgrund bzw. Ursache stiller Reserven

\begin{tabular}{|l|l|l|}
\hline Wertansatz & Art der Reserven & Grundlage \\
\hline Tatsächlicher Wert & & \\
\hline Gesetzlicher Höchstwert & & $\begin{array}{l}\text { Kostenwertprinzip, Art. 960a } \\
\text { Abs. 1 und 2 OR }\end{array}$ \\
\hline Vorsichtig festgelegter Wert & Ermessensreserven & $\begin{array}{l}\text { Vorsichtsprinzip, Art. 958c } \\
\text { Abs. 1 Ziff. 5 OR }\end{array}$ \\
\hline Finanzieller Buchwert & & $\begin{array}{l}\text { Art. 960a Abs. 4 und 960e } \\
\text { Abs. 3 und 4 OR }\end{array}$ \\
\hline
\end{tabular}

Beispielbuchungen:

\begin{tabular}{|lllll|r|}
\hline 1500 & Sachanlagen & an & 8510 & A.o. Ertrag & 50 '000 \\
\hline 1480 & Vorräte & an & 4000 & Waren-, Materialaufwand & $50^{\prime} 000$ \\
\hline 2600 & Rückstellungen & an & $6^{\star \star \star}$ & Verschiedene ER-Konten & $50^{\prime} 000$ \\
\hline
\end{tabular}

Rangrücktritt (Art. 725 Abs. 2 OR bzw. Art. 725b Abs. 4 Ziff. 1 nOR)

a) Gesellschaftsgläubiger treten im Umfang der Überschuldung hinter alle anderen Gesellschaftsgläubiger zurück und stunden ihre Forderungen, sofern der Rangrücktritt den geschuldeten Betrag und die Zinsforderungen während der Dauer der Überschuldung umfasst;

b) $\mathrm{Zu}$ beachten ist, dass die Überschuldung bestehen bleibt und nur der Gang zum Richter vermieden wird. 
Beispielbuchungen:

$2400 \quad$ Fremdkapital

an 2490

Fremdkapital mit Rangrücktritt

$50 ’ 000$

Das Fremdkapital mit Rangrücktritt ist nicht zwingend in der Bilanz separat auszuweisen. Ohne Ausweis in der Bilanz ist der Rangrücktritt im Anhang offen zu legen.

S. dazu auch die neuere Praxis des Bundesgerichts in BGer 4A.277/2010 vom 2. September 2010 (dem Beschwerdeführer kann nicht gefolgt werden, wenn er vorträgt, durch einen Rangrücktritt werde die Substanz für den Insolvenzfall erhöht und dadurch der von den Gläubigern erlittene Schaden reduziert bzw. beseitigt; der Rangrücktritt hat keinen Einfluss auf die Aktiven; er kann gegebenenfalls Ausfälle anderer Gläubiger verhindern oder verringern, da der Rangrücktritt unter anderem die Erklärung des Rangrücktrittsgläubigers enthält, im Falle eines Konkurses im Rang hinter alle übrigen Gläubiger bis zu deren vollen Befriedigung zurückzutreten; die Rangrücktrittserklärung beinhaltet jedoch keinen Forderungsverzicht; der Beschwerdeführer verkennt mit seinen Vorbringen insbesondere, dass im zu beurteilenden Fall nicht der Schaden einzelner Gläubiger zur Diskussion steht, sondern der Reflexschaden der Gläubigergesamtheit, der deckungsgleich ist mit dem aus der Pflichtverletzung der Organe resultierenden Schaden der Gesellschaft [BGE 132 III 342 E. 2.3.3 S. 348; 117 II 432 E. 1b/gg S. 440; Urteil 4C.363/2006 vom 13. März 2007 E. 4.3]; da mit dem Rangrücktritt die betreffenden Schulden der Gesellschaft nicht wegfielen, blieb dieser ohne Einfluss auf das Gesellschaftsvermögen), 4A.391/2009 vom 12. Februar 2010, 4A.188/2008 vom 9. September 2008, 4A.478/2008 vom 16. Dezember 2008 und 4C.58/2007 vom 25. Mai 2007.

Deshalb hat EXPERTsuisse die Rangrücktrittsvereinbarung neu formuliert und die Gesellschaftsgläubiger verzichten bei Konkurseröffnung (Art. 175 und 192 SchKG) und für den Fall der Bestätigung eines Nachlassvertrags mit Vermögensabtretung (Art. 317 SchKG) auf die rangrücktrittsbelasteten Forderungen im Umfang der Unterdeckung. Bestehende Rangrücktritte sind folglich zu überprüfen und allenfalls anzupassen.

\section{Die finanzielle Sanierung}

Die finanzielle Sanierung beschränkt sich nicht auf Buchungsvorgänge, sondern behebt oder lindert zumindest einige der schwerwiegendsten Probleme einer Unternehmenskrise, nämlich einer Illiquidität, durch Verbesserung der finanziellen Strukturen, verbunden i.d.R. mit der Zuführung neuer flüssiger Mittel. 


\section{Veränderung des Eigenkapitals}

a) Vorbemerkung: Lediglich die Herabsetzung des Aktienkapitals ist für sich alleine noch keine finanzielle Sanierung, sondern eine bilanzielle. Steht die Herabsetzung i.V.m. einer gleichzeitigen Heraufsetzung des Aktienkapitals kann der gesamte Vorgang als finanzielle Sanierung eingestuft werden. S. zur Herabsetzung des Aktienkapitals Art. 732 ff. OR bzw. Art. 653j ff. nOR;

b) Freiwillige Zuzahlung der Aktionäre, sog. à fonds perdu-Zahlungen. Hinsichtlich Zuweisung solcher Einlagen und Zuschüsse in die gesetzliche Kapitalreserve s. Art. 671 Abs. 1 Ziff. 1 nOR;

c) Aktienkapitalerhöhungen mit Ausgabe von Vorzugsaktien (Art. 654 ff. OR bzw. Art. 650 Abs. 2 Ziff. 2 nOR), ev. auch von Genussscheinen (Art. 657 OR).

Beispielbuchungen Grundkapitalerhöhung:

\begin{tabular}{|c|c|c|c|c|}
\hline 0 & Flüssige Mittel & an 2800 & Grundkapital & $100 ' 000$ \\
\hline 1020 & Flüssige Mittel & an 2900 & Kapitalreserve - Agio & $20 ' 000$ \\
\hline
\end{tabular}

Beispielbuchungen à fonds perdu-Zahlung:

\begin{tabular}{|lrrll|}
\hline 1020 & Flüssige Mittel & an 2900 & Kapitalreserve & $100^{\prime} 000$ \\
\hline 1020 & Flüssige Mittel & an 2970 & Bilanzverlust & 100 '000 \\
\hline \multicolumn{7}{r}{} & oder & & \\
\hline 1020 & Flüssige Mittel & an 2900 & Kapitalreserve & $2000^{\prime} 000$ \\
\hline
\end{tabular}

Gestützt auf Art. 20 Abs. 3 DBG, Art. 7 Abs. 1bis StHG und den entsprechenden kantonalen Bestimmungen, bspw. § 29 Abs. 3 StG AG und $\S 20$ Abs. 3 StG ZH i.V.m. den gesellschaftsrechtlichen Normen, namentlich Art. 959a Abs. 2 Ziff. 3. OR, sind Kapitalreserven offen auszuweisen, damit diese zu einem späteren Zeitpunkt steuerfrei zurückbezahlt werden können. Entsprechend wird ein Bilanzverlust nicht verrechnet, sondern weiter ausgewiesen.

Buchungen à fonds perdu-Zahlung Dritte:



\section{Veränderung des Fremdkapitals}

a) Umwandlung von kurz- und langfristigem Fremdkapital in Eigenkapital;

b) Forderungsverzicht (Nachlassverträge Prozent- und Liquidationsvergleich, aussergerichtlich); 
c) Umwandlung von kurz- in langfristiges Fremdkapital (keine eigentliche Sanierungswirkung);

d) Erhalt von Aktionärsdarlehen (keine eigentliche Sanierungswirkung).

Beispielbuchungen Umwandlung FK in EK:

\begin{tabular}{|llll|l|}
\hline 2400 & $\begin{array}{l}\text { Kurz- oder langfristiges } \\
\text { Fremdkapital }\end{array}$ & an 1020 & Flüssige Mittel & 100 '000 \\
\hline 1020 & Flüssige Mittel & an 2800 & Grundkapital, Reserven & 100 '000 \\
\hline
\end{tabular}

Beispielbuchungen Forderungsverzicht:

\begin{tabular}{|llrll|l|}
\hline 2480 & Flüssige Mittel & an 8510 & A.o. Ertrag & 100 '000 \\
\hline & & oder & & \\
\hline 2480 & Aktionärsdarlehen & an 2900 & Kapitalreserve & $1000^{\prime} 000$ \\
\hline 2400 & Fremdkapital Dritte & an 8510 & A.o. Ertrag & $1000^{\prime} 000$ \\
\hline
\end{tabular}

Beispielbuchungen kFK und IFK und Erhalt:

\begin{tabular}{|lllll|l|}
\hline 2000 & Kurzfristiges Fremdkapital & an & 2400 & Langfristiges Fremdkapital & 100 '000 \\
\hline 1020 & Flüssige Mittel & an & 2480 & Aktionärsdarlehen & 100 '000 \\
\hline
\end{tabular}

\section{Die organisatorische Sanierung}

Die organisatorische Sanierung beschränkt sich, wie die finanzielle Sanierung nicht auf Buchungsvorgänge, sondern behebt oder verbessert organisatorische Mängel bezogen auf die Aufbau- und/oder Ablauforganisation. Sie bildet die fundamentalste Sanierungsart. S. dazu oben Kapitel Sanierungsarten und -formen bzw. -massnahmen.

\section{Bilanzielle Sanierung}

Die Charakteristik der bilanziellen Sanierung liegt darin, dass keine finanziellen Vorgänge notwendig sind.

Es sind nebst den für die Durchführung teilweise notwendigen organisatorischen Vorkehrungen lediglich entsprechende Buchungen zu treffen.

Die organisatorische Sanierung bedient sich gerne des Benchmarking und steht bspw. in Verbindung mit

a) neuen oder der Erweiterung von bestehenden Geschäftsfeldern;

b) Personalmassnahmen;

c) Investitionsaufschub

d) Reduktion bzw. Aufgabe Betriebs- und Verwaltungsräumlichkeiten sowie Aufgabe Standorte 
e) Rationalisierungen Produktion, Vertrieb, Materialwirtschaft

\section{V. Überschuldungsprüfung und Sanierungsplan, Vorgehen der Revisionsstelle bei Überschuldung}

Die Überschuldungsprüfung gilt für alle Kapitalgesellschaften, unabhängig davon ob ordentlich, eingeschränkt geprüft oder gar nicht zu prüfen.

Sie gilt auch mit Bezug auf subsidiären Handlungs- und Anzeigepflichten der Revisionsstelle in den Fällen eines Kapitalverlusts und einer offensichtlichen Überschuldung.

Bei Unternehmensgruppen wird immer Einzelabschluss geprüft. Der Konzernrechnung kommt keine Bedeutung zu. Vorsicht ist hinsichtlich Dominoeffekt angebracht, insbesondere bezüglich Prinzipalstrukturen und Konzernen mit zentralisiertem Liquiditätsmanagement, sog. Cash-Pooling.

Nebst Gesetz/en und Rechtsprechung sind durch Revisionsstelle zu beachten:

- Standard zur eingeschränkten Revision (SER): Ziff. 9 und Anhang H „Verhalten bei Überschuldung“ sowie Anhang $\mathrm{G}$ „Unternehmensfortführung“;

- PS 290 „Pflichten der gesetzlichen Revisionsstelle bei Kapitalverlust und Überschuldung";

- Schweizer Prüfungshinweis (PH) 10 „Berichterstattung zur Prüfung von besonderen Vorgängen“, insbesondere Berichtsvorlagen zur Prüfung der Zwischenbilanz zu Fortführungs- und zu Veräusserungswerten (Art. 725 Abs. 2 OR).

Die nachfolgenden Übersichten zeigen das Vorgehen der Revisionsstelle bei Überschuldung. 


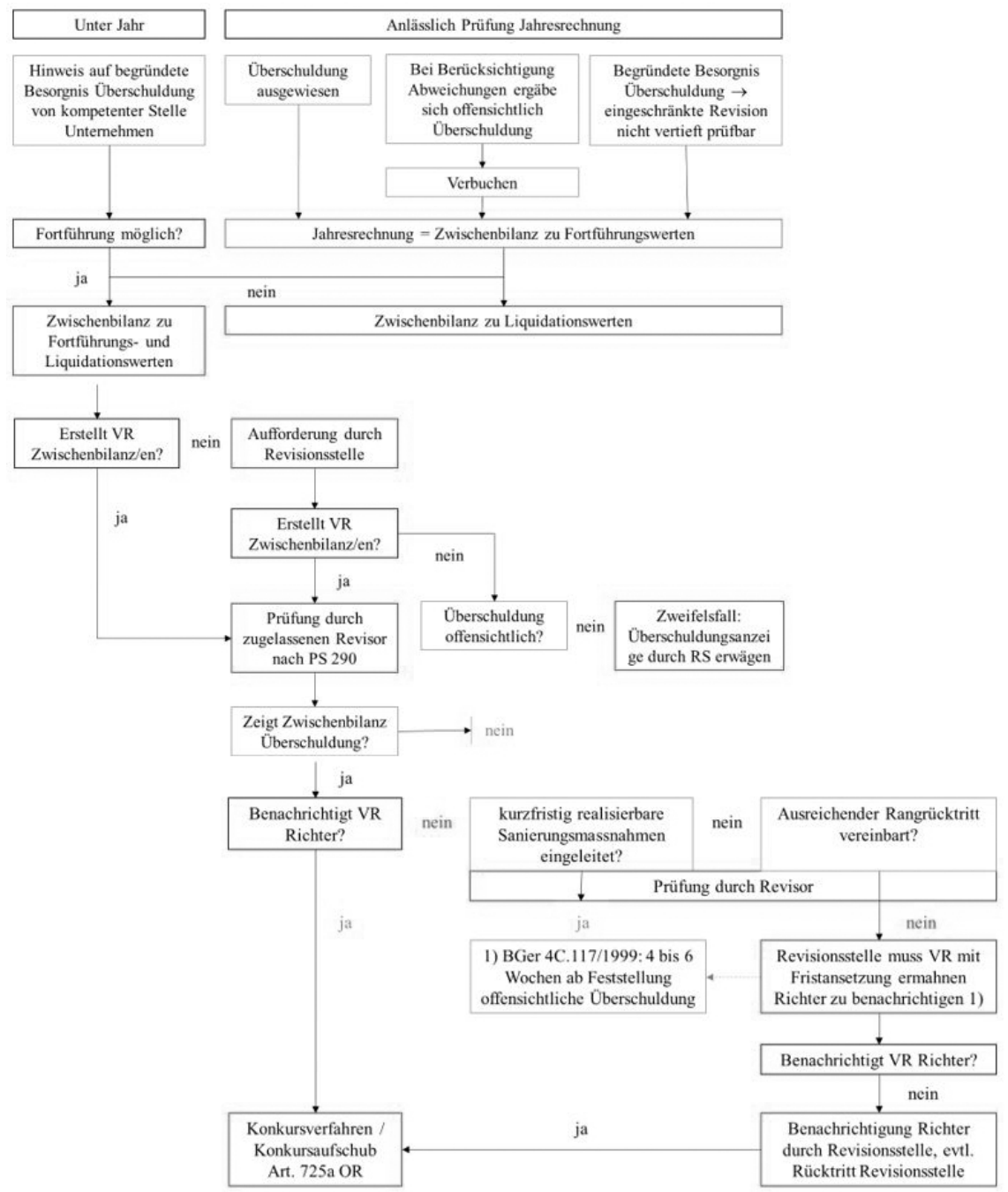

\section{Steuerfolgen der Sanierungsmassnahmen}

Die Sanierungsmassnahmen beschlagen verschiedene Steuerarten. Hier werden einige wesentliche steuerliche Aspekte aufgezeigt, indessen ohne Anspruch auf Vollständigkeit.

Die steuerliche Betrachtung erfolgt nachstehend aus der Sicht des zu sanierenden Unternehmens und aus jener der daran Beteiligten. 


\section{Steuerfolgen aus Sicht des zu sanierenden Unternehmens}

Die steuerlichen Aspekte bei der Sanierung im Rahmen der Abschlussgestaltung des zu sanierenden Unternehmens beziehen sich auf die Frage, ob die Sanierungsmassnahmen steuerlich erfolgswirksam oder erfolgsneutral sind bzw. echte oder unechte oder keine Sanierungsgewinne darstellen.

Voraussetzung für die nachfolgenden Ausführungen ist, dass ein Kapitalunternehmen steuerlich betrachtet sanierungsbedürftig ist, wobei sich die steuerliche Beurteilung an der betriebswirtschaftlichen Sichtweise orientiert. Dies ist folglich dann der Fall, wenn eine echte Unterbilanz vorliegt, das heisst wenn Bilanzverluste bestehen, welche das Kapitalunternehmen weder über offene noch allfällige stille Reserven ausgleichen kann.

Nachstehend werden die vorgängig besprochenen bilanziellen, finanziellen und organisatorischen Sanierungsmassnahmen entsprechend eingeteilt.

\begin{tabular}{|c|c|}
\hline \multicolumn{2}{|c|}{ Sanierungsgewinne } \\
\hline Echte & $\begin{array}{l}\text { - bilanzielle } \\
\text { - Aufwertung von Aktiven } \\
\text { - Auflösung von stillen Reserven } \\
\text { - finanzielle } \\
\text { - à fonds perdu-Zahlungen von Dritten (Nichtbetei- } \\
\quad \text { ligte) } \\
\text { - Forderungsverzichte von Dritten (Nichtaktionäre) } \\
\text { - Forderungsverzichte von Beteiligten }\end{array}$ \\
\hline Unechte & $\begin{array}{l}\text { - bilanzielle } \\
\text { - Kapitalherabsetzung } \\
\text { - finanzielle } \\
\text { - à fonds perdu-Zahlungen von Beteiligten } \\
\text { - Kapitalerhöhung } \\
\text { - Forderungsverzichte von Beteiligten }\end{array}$ \\
\hline Keine & $\begin{array}{l}\text { - bilanzielle } \\
\text { - Rangrücktritt } \\
\text { - finanzielle } \\
\text { - Besserungsscheine } \\
\text { - Sanierungsgenussscheine }\end{array}$ \\
\hline
\end{tabular}


Eine weitere Einteilung kann danach erfolgen, ob die Sanierungsmassnahmen zu einer Veränderung des Eigenkapitals und/oder des Fremdkapitals führen.

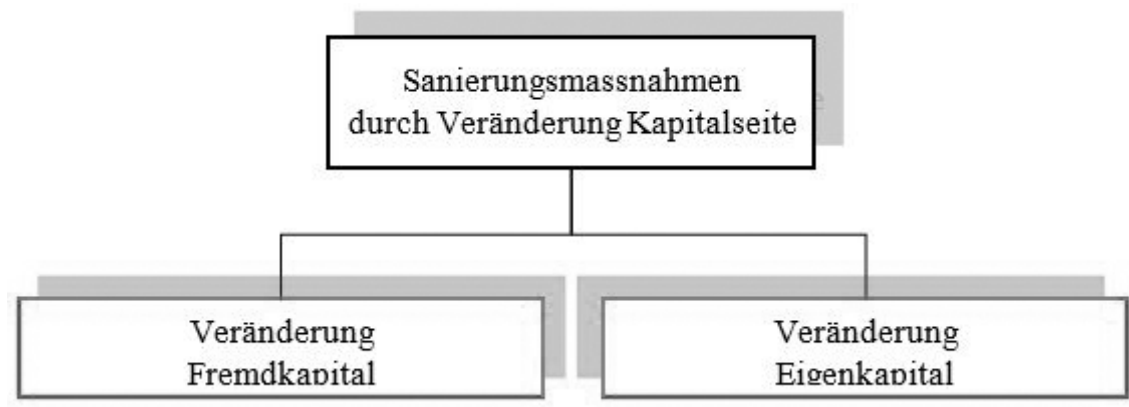

Im Einzelnen zeigt sich diesbezüglich folgendes:

\section{Veränderung Fremdkapital}

\begin{tabular}{|c|c|c|}
\hline Auflösung Reserven & $\rightarrow$ & $\begin{array}{l}\text { - offene Reserven: keine Gewinnsteuer } \\
\text { - } \text { stille Reserven: Gewinnsteuer }\end{array}$ \\
\hline Aufwertung Aktiven & $\rightarrow$ & - Gewinnsteuer \\
\hline Kapitalherabsetzung & $\rightarrow$ & $\begin{array}{ll}\text { - } & \text { keine Gewinnsteuer } \\
\text { - } & \text { bei anschliessender Wiedererhöhung } \\
& \text { allenfalls Emissionsabgabe, Ausnahme } \\
& \text { Härtefälle }\end{array}$ \\
\hline $\begin{array}{l}\text { Forderungsverzicht von } \\
\text { Beteiligten }\end{array}$ & $\rightarrow$ & $\begin{array}{l}\text { - } \text { allenfalls Gewinnsteuer, Ausnahme unter } \\
\text { bestimmten Voraussetzungen } \\
\text { - } \text { allenfalls Emissionsabgabe, Ausnahme } \\
\text { Härtefälle }\end{array}$ \\
\hline $\begin{array}{l}\text { A fonds perdu-Zahlungen von } \\
\text { Beteiligten }\end{array}$ & $\rightarrow$ & $\begin{array}{ll}\text { - } & \text { keine Gewinnsteuer } \\
\text { - } & \text { allenfalls Emissionsabgabe, Ausnahme } \\
& \text { Härtefälle }\end{array}$ \\
\hline
\end{tabular}




\section{Veränderung Eigenkapital}

\begin{tabular}{|c|c|c|}
\hline $\begin{array}{l}\text { Umwandlung kurz- in } \\
\text { langfristiges Fremdkapital }\end{array}$ & $\rightarrow$ & $\begin{array}{ll}\text { - } & \text { keine Gewinnsteuer } \\
\text { - } & \text { bei Ausgabe von bspw. Obligationen, } \\
& \text { Geldmarktpapieren Emissionsabgabe }\end{array}$ \\
\hline $\begin{array}{l}\text { Umwandlung Fremd- in } \\
\text { Eigenkapital }\end{array}$ & $\rightarrow$ & $\begin{array}{l}\text { - keine Gewinnsteuer } \\
\text { Emissionsabgabe }\end{array}$ \\
\hline $\begin{array}{l}\text { Forderungsverzicht von } \\
\text { unabhängigen Dritten }\end{array}$ & $\rightarrow$ & $\begin{array}{ll}\text { - } & \text { Gewinnsteuer } \\
\text { - } & \text { keine Emissionsabgabe }\end{array}$ \\
\hline $\begin{array}{l}\text { Forderungsverzicht von } \\
\text { Beteiligten }\end{array}$ & $\rightarrow$ & $\begin{array}{l}\text { - Gewinnsteuer } \\
\text { - } \quad \text { keine Emissionsabgabe }\end{array}$ \\
\hline
\end{tabular}

Der Forderungsverzicht von Beteiligten ist grundsätzlich gleich zu behandeln wie der Forderungsverzicht von Dritten. D.h. dem Kapitalunternehmen erwächst dadurch ein steuerlich erfolgswirksamer Vermögenszugang. Alle zulasten dieses echten Sanierungsertrags vorgenommenen Verlustausbuchungen, Abschreibungen und Rückstellungen gelten als steuerlich erfolgt.

Im Sinn einer Ausnahme gelten Forderungsverzichte von Beteiligten als steuerlich erfolgsneutral,

- wenn und soweit die Darlehen der Beteiligten vor der Sanierung steuerlich als verdecktes Eigenkapital behandelt worden sind;

- wenn die Darlehen der Beteiligten erstmalig oder zusätzlich wegen schlechten Geschäftsganges gewährt wurden und unter gleichen Umständen von unabhängigen Dritten nicht zugestanden worden wären.

Ab dem 1. Januar 2010 bzw. 2009 gilt das Kapitaleinlageprinzip beim Bund gemäss Art. 20 Abs. 3 DBG und in den Kantonen gemäss Art. 7b StHG. Damit gewinnen Kapitaleinlagen im Zusammenhang mit Sanierungen an Bedeutung und wirken wie folgt.

Kapitaleinlagen von Inhabern von Beteiligungsrechten, namentlich Einlagen, Aufgelder und Zuschüsse, welche im Rahmen einer Sanierung geleistet werden, stellen ebenfalls unechten Sanierungsgewinn dar. Werden sie mit bestehenden Verlusten verrechnet, gelangt der Freibetrag gemäss Art. 6 Abs. 1 Bst. k StG (s. sogleich unten) zur Anwendung. Für Kapitaleinlagen, welche diesen Freibetrag übersteigen, kann, falls die entsprechenden Voraussetzungen erfüllt sind, der Erlass von der Emissionsabgabe gemäss Art. $12 \mathrm{StG}$ geltend gemacht werden. Soweit derartige Kapitaleinlagen nicht durch die Ausbuchung von handelsrechtlichen Verlustvorträgen vernichtet werden, gelten 
diese steuerrechtlich als Reserven aus Kapitaleinlagen gemäss Art. 20 Abs. 3 DBG, Art. 7b StHG bzw. Art. 5 Abs. 1bis VStG und den entsprechenden kantonalen Vorschriften.

Gemäss Art. 6 Abs. 1 Bst. k StG sind von der Emissionsabgabe die bei offenen Sanierungen vorgenommene Begründung von Beteiligungsrechten oder die Erhöhung von deren Nennwert bis zur Höhe vor der Sanierung sowie Zuschüsse von Gesellschaftern oder Genossenschaftern bei stillen Sanierungen ausgenommen, soweit bestehende Verluste beseitigt werden und die Leistungen der Gesellschafter oder Genossenschafter gesamthaft Fr. 10 Millionen nicht übersteigen.

Wenn bei der offenen oder stillen Sanierung einer Aktiengesellschaft, Kommanditaktiengesellschaft, Gesellschaft mit beschränkter Haftung oder Genossenschaft die Erhebung der Emissionsabgabe eine offenbare Härte bedeuten würde, so soll gemäss Art. $12 \mathrm{StG}$ die Abgabe gestundet oder erlassen werden.

Für Stundung bzw. Erlass der Emissionsabgabeforderung bei der offenen oder stillen Sanierung sind gemäss Art. $17 \mathrm{StV}$ ein Gesuch zu stellen. Das Gesuch ist bei der ESTV einzureichen und hat die Ursachen der Verluste und die zu ihrer Beseitigung getroffenen und vorgesehenen Massnahmen darzustellen. Unterlagen über die Sanierung, wie Rundschreiben, Rechenschaftsberichte, Generalversammlungsprotokolle sowie die Geschäftsberichte oder Jahresrechnungen der letzten Jahre und eine Aufstellung der Sanierungsbuchungen sind beizulegen. Mit besonderem amtlichem Formular sind die von den Gesellschaftern oder Genossenschaftern bei der Sanierung erlittenen Einbussen und ihre in Beteiligungsrechte umgewandelten Forderungen zu melden. Die ESTV kann vom Gesuchsteller über alle Tatsachen, die für die Stundung oder den Erlass von Bedeutung sein können, die erforderlichen Auskünfte und Belege verlangen. Erfüllt der Gesuchsteller die Auflage nicht, so wird das Gesuch abgewiesen.

Es zeigen sich folgende steuerlich unterschiedliche Sanierungsgewinne:

\section{Sanierungsgewinne}

Je nachdem, ob ein echter, unechter oder kein Sanierungsgewinn vorliegt, zeigen sich folgende unterschiedliche steuerliche Folgen:

- Echter Sanierungsgewinn Der echte Sanierungsgewinn ist steuerlich erfolgswirksam. D.h., dass Abschreibungen, Bildungen von Rückstellungen und Verlustausbuchungen als steuerlich vorgenommen gelten. Die Handelsbilanz entspricht damit der Steuerbilanz. 
- Unechter Sanierungsgewinn

Der unechte Sanierungsgewinn ist steuerlich erfolgsneutral. D.h., dass Abschreibungen, Bildungen von Rückstellungen und Verlustausbuchungen als steuerlich nicht erfolgt gelten. Handelsrechtlich wurde saniert, steuerlich können diese Buchungen hingegen später steuerwirksam nachgeholt werden. Damit wird die Führung einer Steuerbilanz notwendig.

- Kein Sanierungsgewinn

Eine weitere Einteilung kann danach erfolgen, ob die Sanierungsmassnahmen zu einer Veränderung des Eigenkapitals und/oder des Fremdkapitals führen.

Im Zusammenhang mit dem Kapitaleinlageprinzip sind zwei weitere Bestimmungen von Bedeutung, nämlich Art. 125 Abs. 3 DBG und Art. 5 Abs. $1^{\text {bis }}$ VStG. Zentral ist dabei, dass Kapitalunternehmen sowohl für die direkte Bundessteuer als auch für die Verrechnungssteuer die aus Kapitaleinlagen, Aufgeldern und Zuschüssen resultierenden Reserven auf gesonderten Eigenkapitalkonten in ihrer Handelsbilanz ausweisen müssen, um vom Kapitaleinlageprinzip profitieren zu können.

Das Bilanzrecht hat indessen für Kapitalunternehmen bis 31. Dezember 2012 bzw. 2014 keine solche Unterteilung vorgesehen, sondern die Aufteilung des Eigenkapitals gemäss Art. $671 \mathrm{ff}$. OR in Grundkapital, allgemeine, Aufwertungsund Reserven für eigene Aktien und Bilanzgewinn bzw. -verlust sowie allfälligen weiterführenden statutarischen und freien Reserven verlangt.

Es empfiehlt sich daher, in Anlehnung an die Struktur in der Konzernrechnung, das Eigenkapital von Kapitalunternehmen bspw. wie nachstehend dargestellt zu unterteilen.

Die Gliederung des Eigenkapitals bis Ende 2012 gemäss Art. 663a aOR (aufgehoben ab 2013) zeigt sich wie folgt. 


\begin{tabular}{|l|}
\hline Grundkapital \\
\hline Kapitalreserven \\
Gesetzliche Reserven (Kapitaleinlageprinzip bei Art. 671 Abs. 2 Ziff. 1 OR = Aufgel- \\
der bzw. Agios) \\
Weitere Einlagen, Zuschüsse (Kapitaleinlageprinzip) \\
Gewinnreserven \\
\hline Gesetzliche Reserven (allgemeine, eigene Aktien, Aufwertung) \\
\hline Statutarische Reserven \\
\hline Freie Reserven \\
\hline Bilanzgewinn bzw. - verlust
\end{tabular}

Ab 1. Januar 2013 bzw. 2015 erfolgt die Gliederung des Eigenkapitals gemäss Art. 959a Abs. 2 Ziff. 3. OR:

\begin{tabular}{|c|}
\hline Grundkapital \\
Gesetzliche Reserven \\
\hline Kapitalreserven \\
\hline Gewinnreserven \\
\hline Freiwillige Reserven \\
\hline Gewinnreserven \\
\hline Statutarische Reserven \\
\hline Freie Reserven \\
\hline Bilanzgewinn bzw. -verlust \\
\hline Eigene Kapitalanteile als Minusposition
\end{tabular}


$\mathrm{S}$ u.a. die folgende Rechtsprechung zur Sanierung aus Sicht des zu sanierenden Kapitalunternehmens BGer vom 20. Oktober 2014, StE 2015 DBG/ZH B 72.19 Nr. 19 (steuerliche Beurteilung von Forderungsverzichten; bei einer Fusion zweier Kapitalgesellschaften kann die aufnehmende Gesellschaft die Verlustvorträge der absorbierten Gesellschaft an sich steuerwirksam geltend machen; handelt es sich bei der absorbierten Gesellschaft um ein Unternehmen, das vor der Fusion saniert worden ist, ist für die Zulassung der Verlustvorträge bei der aufnehmenden Gesellschaft allerdings zu prüfen, welche Sanierungsleistung erbracht und wie diese steuerlich zu behandeln ist; nach der in der Lehre vorherrschenden Auffassung sollen Sanierungsleistungen von Aktionären, insbesondere auch Forderungsverzichte, nicht Ertrag, sondern Eigenkapital- oder Fremdkapitaleinlagen sein, die - entgegen Forderungsverzichten seitens an der Gesellschaft nicht beteiligter Gläubiger - nicht in die Erfolgsrechnung gehörten; es sei jede Sanierungsleistung eines Beteiligten als erfolgsneutraler Wertzugang zu behandeln; das Bundesgericht teilt diese Auffassung nicht und geht in Bezug auf die beiden strittigen Forderungsverzichte von erfolgswirksamen Vorgängen aus, die eine Verrechnung im Sinn von Art. 67 DBG ausschliessen), BGer vom 2. September 1989, StE 1990 BdBSt B 72.16 Nr. 1 (Forderungsverzicht durch Aktionäre [Art. 49 Abs. 1 Buchst. a BdBSt]; Forderungsverzichte, gleichgültig, ob der Verzicht von einem Aktionär oder einem unbeteiligten Gläubiger ausgesprochen wurde, sind in der Regel echte Sanierungsgewinne und deshalb ertragswirksame Vermögenszugänge; sie werden nur dann dem Ertrag nicht zugerechnet, wenn und soweit es sich entweder um eine Darlehensforderung handelt, die vor der Sanierung steuerlich als verdecktes Eigenkapital behandelt wurde, oder um Aktionärsdarlehen, die erstmalig oder zusätzlich wegen schlechten Geschäftsganges gewährt wurden und die unter den gleichen Umständen von unabhängigen Dritten nicht zugestanden worden wären; die Auffassung, es sei jede Sanierungsleistung eines Beteiligten ohne Rücksicht auf die Form [Kapitaleinzahlung oder Forderungsverzicht] als erfolgsneutraler Wertzugang zu behandeln, kann nicht geteilt werden), BGer vom 13. August 2008, StE 2009 DBG/ZH B 21.1 Nr. 18 (Forderungsverzicht im Privatvermögen; der Erlass von Geschäftsschulden bildet Einkommen aus selbständiger Erwerbstätigkeit; der Forderungsverzicht gegenüber einer Privatperson stellt nach der Reinvermögenszugangstheorie eine Einkunft im Sinn der Einkommensgeneralklausel dar; ein steuerfreier privater Kapitalgewinn liegt nicht vor; in casu erfassten die Vorinstanzen lediglich ein Einkommen in der Höhe der Werthaltigkeit der Forderung im Zeitpunkt des Verzichts; ob allenfalls der volle Nennwert der erlassenen Schuld einkommenssteuerrechtlich zu erfassen gewesen wäre, hat das Bundesgericht nicht geprüft, da es nicht über die Begehren der Parteien hinausgehen darf; immerhin hat es festgestellt, dass die von den Steuerbehörden 
praktizierte Lösung mit Blick auf den Grundsatz der Realität des Einkommens durchaus als vertretbar erscheint; andernfalls müsste beispielsweise bei jedem Steuererlass konsequenterweise - im Ausmass des erlassenen Steuerbetrags - ein steuerbares Einkommen aufgerechnet werden), StGer SO vom 13. Januar 1992, StE 1992 SO B 72.13.1 Nr. 1 (der Forderungsverzicht einer Kapitalgesellschaft gegenüber einer Tochtergesellschaft ist als verdeckte Kapitaleinlage a] steuerlich zu berichtigen, wenn eine Steuerumgehung vorliegt, b] nicht zu berichtigen, wenn der Forderungsverzicht durch kaufmännische oder wirtschaftliche Erwägungen vollauf erklärbar ist; Abschreibungen sind a] für die in der Berechnungsperiode effektiv eingetretenen Wertverminderungen vorzunehmen, b] sie können nachgeholt werden, wenn andernfalls die Höchstwertvorschriften verletzt würden oder wenn das Nachholen dem Gedanken der Besteuerung nach der wirtschaftlichen Leistungsfähigkeit entspricht).

\section{Steuerfolgen aus Sicht der Beteiligten}

Aus der Sicht der das Unternehmen sanierenden Beteiligten ist für die Ermittlung der steuerlichen Folgen der Sanierungsmassnahmen wesentlich, ob die Beteiligungsrechte des sanierungsbedürftigen Unternehmens bei diesen

- Geschäftsvermögen oder

- Privatvermögen

darstellen. 


\begin{tabular}{|c|c|c|}
\hline \multirow[t]{2}{*}{ Sanierungsmassnahme } & \multicolumn{2}{|c|}{ Beteiligungsrechte stellen dar } \\
\hline & Geschäftsvermögen & Privatvermögen \\
\hline Kapitalherabsetzung & $\begin{array}{l}\text { Steuerlich erfolgswirk- } \\
\text { same Abschreibung der } \\
\text { Beteiligung im Umfang der } \\
\text { Herabsetzung }\end{array}$ & $\begin{array}{l}\text { Kapitalverlust (nicht reali- } \\
\text { siert) auf Privatvermögen } \\
\text { steuerlich nicht abzugsfä- } \\
\text { hig }\end{array}$ \\
\hline Kapitalerhöhung & $\begin{array}{l}\text { Steuerlich erfolgsunwirk- } \\
\text { same Aktivierung auf der } \\
\text { Beteiligung im Umfang der } \\
\text { Erhöhung, ggf. kombiniert } \\
\text { mit einer erfolgswirksa- } \\
\text { men Wertberichtigung }\end{array}$ & $\begin{array}{l}\text { Keine steuerliche Abzugs- } \\
\text { fähigkeit der à fonds } \\
\text { perdu-Zahlung; daher ein- } \\
\text { kommensunwirksam; } \\
\text { allenfalls höherer Vermö- } \\
\text { genssteuerwert }\end{array}$ \\
\hline A fonds perdu-Zahlung & Do. Kapitalerhöhung & Do. Kapitalerhöhung \\
\hline Aufwertung von Aktiven & $\begin{array}{l}\text { Keine Aktivierungspflicht } \\
\text { der Beteiligung im Umfang } \\
\text { der Aufwertung, da Wert } \\
\text { vorher schon vorhanden }\end{array}$ & $\begin{array}{l}\text { Kein realisierter Kapital- } \\
\text { gewinn, allenfalls höherer } \\
\text { Vermögenssteuerwert }\end{array}$ \\
\hline $\begin{array}{l}\text { Auflösung von stillen } \\
\text { Reserven }\end{array}$ & $\begin{array}{l}\text { Do. Aufwertung von Akti- } \\
\text { ven }\end{array}$ & $\begin{array}{l}\text { Do. Aufwertung von Akti- } \\
\text { ven }\end{array}$ \\
\hline Rangrücktritt & $\begin{array}{l}\text { Keine Änderung des Betei- } \\
\text { ligungswerts; steuerlich } \\
\text { erfolgswirksame oder } \\
\text {-unwirksame (verdecktes } \\
\text { Eigenkapital) Abschrei- } \\
\text { bung der Forderung im } \\
\text { Umfang der Verlustwahr- } \\
\text { scheinlichkeit oder Bil- } \\
\text { dung einer entsprechen- } \\
\text { den Rückstellung }\end{array}$ & $\begin{array}{l}\text { Einkommensunwirksam; } \\
\text { Vermögenssteuerwert } \\
\text { wird nicht tangiert }\end{array}$ \\
\hline Forderungsverzicht & $\begin{array}{l}\text { Abschreibung Forderung } \\
\text { und Aktivierung Beteili- } \\
\text { gung im Umfang des For- } \\
\text { derungsverzichts, ggf. } \\
\text { kombiniert mit einer } \\
\text { erfolgswirksamen Wertbe- } \\
\text { richtigung } \\
\text { Alternative: Direkte } \\
\text { Abschreibung der Forde- } \\
\text { rung }\end{array}$ & $\begin{array}{l}\text { Analog zur allfälligen } \\
\text { Besteuerung des entspre- } \\
\text { chenden Forderungsver- } \\
\text { zichts steuerlich abzugsfä- } \\
\text { hig (s. BGer vom 13. August } \\
\text { 2008, StE 2009 DBG/ZH B } \\
\text { 21.1 Nr. 18); ansonsten } \\
\text { steuerlich nicht abzugsfä- } \\
\text { higer, realisierter Kapital- } \\
\text { verlust auf Privatvermö- } \\
\text { gen; allenfalls höherer } \\
\text { Vermögenssteuerwert }\end{array}$ \\
\hline
\end{tabular}




\begin{tabular}{|l|l|l|}
\hline Besserungsschein & $\begin{array}{l}\text { Steuerlich erfolgswirk- } \\
\text { same oder -unwirksame } \\
\text { (verdecktes Eigenkapital) } \\
\text { Abschreibung der Forde- } \\
\text { rung; keine Aktivierung } \\
\text { des Besserungsscheins. }\end{array}$ & $\begin{array}{l}\text { Steuerlich nicht abzugsfä- } \\
\text { higer Kapitalverlust; kein } \\
\text { Vermögenssteuerwert des } \\
\text { Besserungsscheins }\end{array}$ \\
\hline Sanierungsgenussschein & $\begin{array}{l}\text { Do. Besserungsschein; } \\
\text { Aktivierung und Bewer- } \\
\text { tung je nach Fortgang } \\
\text { Kapitalunternehmen }\end{array}$ & $\begin{array}{l}\text { Do. Besserungsschein; } \\
\text { Vermögenssteuerwert }\end{array}$ \\
\hline
\end{tabular}

Nachfolgend wird lediglich noch die Verlustverrechnung im Rahmen einer Sanierung dargestellt.

Grundlage dafür bilden Art. 67 Abs. 2 DBG, Art. 25 Abs. 3 StHG und den entsprechenden kantonalen Bestimmungen, bspw. § 74 Abs. 2 StG AG und $\S 70$ Abs. 2 StG ZH, wonach mit Leistungen zum Ausgleich einer Unterbilanz im Rahmen einer Sanierung, die nicht Kapitaleinlagen einschliesslich Aufgelder und Leistungen à fonds perdu von Mitgliedern von Kapitalunternehmen sind, auch Verluste verrechnet werden können, die in früheren Geschäftsjahren entstanden und noch nicht mit Gewinnen verrechnet werden konnten. S. dazu einleitend zur Sanierung oben Kapitel Sanierung.

Entscheidend für die Frage der Verlustverrechnung ist mithin, ob echte, unechte oder keine Sanierungsgewinne vorliegen. S. dazu oben in diesem Kapitel.

Darauf gestützt können mit echten Sanierungsgewinnen auch Verluste verrechnet werden, die vor der siebenjährigen Verlustverrechnungsperiode und vice versa mit unechten Sanierungsgewinnen nur Verluste, die innerhalb der siebenjährigen Verlustverrechnungsperiode entstanden sind.

$\mathrm{S}$ u.a. die folgende Rechtsprechung zur Sanierung aus Sicht der Beteiligten des zu sanierenden Kapitalunternehmens BGer vom 12. August 2008, StE 2008 DBG/SO A 12 Nr. 18 (Honorarverzicht gegenüber sanierungsbedürftiger AG; auch eine unabhängige Drittperson, welche gegenüber einer AG sowohl eine Darlehensforderung als auch eine Forderung aus Honorar hätte, würde im Fall der eingeschränkten Zahlungsfähigkeit der AG auf die Amortisation des Darlehens und nicht auf Zahlung der Honorarforderung bestehen, da auf der Honorarzahlung Einkommens- und allenfalls Mehrwertsteuern zu begleichen sind; der Beschwerdeführer hat sich deshalb im Drittvergleich durchaus rational verhalten; es besteht keine Rechtspflicht des Aktionärs, durch besondere Leistungen oder Verzichte zur Sanierung der AG beizutragen; eine Steuerumgehung liegt demnach nicht vor; zu prüfen ist aber, ob die Abschreibung 
der Honorarforderung im Geschäftsvermögen des Aktionärs geschäftsmässig begründet war; das ist zu bejahen, denn der Sanierungsbedarf der AG ist ausgewiesen und ein Forderungsverzicht ist auch gegen unabhängigen Dritten bei Insolvenz geschäftsmässig begründet; dass der Beschwerdeführer gleichzeitig eine Darlehensamortisation in gleicher Höhe vornahm, ändert daran nichts; auch kann keine Rede davon sein, dass die Umbuchung der Honorarzahlung auf Darlehensrückzahlung bei der AG unzulässig war), VGer ZH vom 14. Juli 1999, StE 1999 ZH B 23.45.2 Nr. 1 (Forderungsverzicht eines Architekten gegenüber einer selbstbeherrschten Gesellschaft; Zuordnung von Beteiligungen zum Privat- oder Geschäftsvermögen; ob der Forderungsverzicht gegenüber einem Dritten geschäftsmässig begründet ist, beurteilt sich danach, ob sich der selbständig Erwerbende aus dem Verzicht in guten Treuen einen seinem Geschäft zukommenden Erfolg versprechen durfte; Gleiches gilt, wenn der Forderungsverzicht gegenüber einer Gesellschaft erfolgt, an welcher der selbständig Erwerbende eine Beteiligung hält, die seinem Privatvermögen zuzuordnen ist; doch ist der Verzicht nicht geschäftsmässig begründet, wenn er seinen Grund im Beteiligungsverhältnis und nicht in der selbständigen Erwerbstätigkeit hat, was im vorliegenden Fall angenommen wurde; beherrschende Beteiligungen eines selbständig Erwerbenden an einem seinem Geschäftsbereich nahestehenden Unternehmen stellen jedenfalls dann Geschäftsvermögen dar, wenn sie wirtschaftlich betrachtet einer Erweiterung des Betriebs des selbständig Erwerbenden gleichkommen; die Mehrheitsbeteiligung an einem Bau- und Immobilienunternehmen wurde in casu nicht als Geschäftsvermögen eines Architekten betrachtet, weil die wirtschaftlichtechnische Verknüpfung der beiden Geschäftsbereiche nicht hinreichend erstellt war).

In Ergänzung die entsprechenden gesetzlichen Bestimmungen von Art. 67 DBG und Art. 25 Abs. 2 und 3 StHG Verluste:

${ }^{1}$ Vom Reingewinn der Steuerperiode können Verluste aus sieben der Steuerperiode (Art. 79 DBG) vorangegangenen Geschäftsjahren abgezogen werden, soweit sie bei der Berechnung des steuerbaren Reingewinnes dieser Jahre nicht berücksichtigt werden konnten.

2 Mit Leistungen zum Ausgleich einer Unterbilanz im Rahmen einer Sanierung, die nicht Kapitaleinlagen nach Art. 60 Bst. a DBG sind, können auch Verluste verrechnet werden, die in früheren Geschäftsjahren entstanden und noch nicht mit Gewinnen verrechnet werden konnten.

Gemäss Art. 18 Abs. 2 Bst. e MWSTG gelten mangels Leistung namentlich die folgenden Mittelflüsse nicht als Entgelt: Einlagen in Unternehmen, insbesondere zinslose Darlehen, Sanierungsleistungen und Forderungsverzichte. 
Beispiele:

Kapitaleinlagen (Einlagen in Einzelfirmen, einfache Gesellschaften, Personengesellschaften, Kapitalgesellschaften und Genossenschaften durch deren Eigentümer, Gesellschafter oder Genossenschafter); Aufgelder, Zuschüsse, Forderungsverzichte im Zusammenhang mit Darlehen; Forderungsverzichte auf Darlehen gegenüber einer überschuldeten Gesellschaft; Zinsverzichte und Zinsreduktionen auf gewährten Darlehen; spezielle Zinskonditionen im Zusammenhang mit Kundenbeziehungen; Darlehenserlass im Rahmen einer Neuausrichtung der Kundenbeziehungen; Einräumung von Baurechten ohne oder mit reduziertem Baurechtszins.

Keine Einlagen sind Beiträge Dritter, die nicht am Unternehmen beteiligt sind. Hierbei handelt es sich entweder um Spenden (bei Zuwendungen Privater), oder um Subventionen beziehungsweise andere öffentlich-rechtliche Beiträge (bei Zuwendungen von Gemeinwesen).

\section{Sanierungsmassnahmen bei Tochter- und Schwestergesellschaften}

Halten Sanierungsmassnahmen bei Tochter- und Schwestergesellschaften dem Drittvergleich stand, gelten die analogen Überlegungen wie bei unabhängigen Dritten.

Zuschüsse von Mutter- bzw. Grossmuttergesellschaften an Tochter- bzw. Enkelgesellschaften werden in der Bilanz der leistenden Gesellschaft auf dem Beteiligungskonto aktiviert und erhöhen damit auch deren Gestehungskosten, ausser sie würden der reinen Werterhaltung dienen. In diesem Fall stellen sie bei der Muttergesellschaft steuerlich abzugsfähigen Aufwand dar, auch wenn bei der Tochtergesellschaft der Zuschuss eine gewinnsteuerneutrale Kapitaleinlage bildet.

À fonds perdu-Zahlungen stellen bei der Tochtergesellschaft gewinnsteuerneutrale Kapitaleinlagen dar. Ein Forderungsverzicht ist indessen grundsätzlich ein echter Sanierungsgewinn, ausser das Darlehen sei vor dem Verzicht als verdecktes Eigenkapital behandelt worden oder sei erstmalig bzw. zusätzlich wegen schlechten Geschäftsganges gewährt und wäre unter gleichen Umständen von unabhängigen Dritten nicht zugestanden worden.

Bei Sanierungsmassnahmen zwischen Schwestergesellschaften basierend auf einem Darlehensverhältnis ist ebenfalls zu unterscheiden zwischen den beiden Situationen, da die Sanierungsmassnahme dem Drittvergleich standhält oder nicht. 
- Sanierungsmassnahme hält Drittvergleich stand

a) Die Schwestergesellschaft, welche auf die Forderung verzichtet, kann diese steuerlich wirksam abschreiben.

b) Die vom Forderungsverzicht profitierende Schwestergesellschaft verbucht einen entsprechenden Ertrag, der als echter Sanierungsgewinn gilt. Sie kann die Verlustverrechnung gestützt auf Art. 67 Abs. 2 DBG bzw. Art. 25 Abs. 3 StHG geltend machen.

c) Bei der gemeinsamen Muttergesellschaft zeigt die Sanierungsmassnahme keine Wirkung, weder in Bezug auf die Gewinnsteuerwerte der Beteiligungen, noch bezüglich deren Gestehungskosten.

- Sanierungsmassnahme hält Drittvergleich nicht stand

Basiert die Sanierungsmassnahme auf dem gemeinsamen Beteiligungsverhältnis der Muttergesellschaft, gelangt entweder die einfache oder die modifizierte Dreieckstheorie zur Anwendung.

a) Einfache Dreieckstheorie

Danach gilt der Forderungsverzicht bei der leistenden Schwestergesellschaft nicht als steuerlich abzugsfähiger Aufwand und bei der Muttergesellschaft als Beteiligungsertrag sowie bei der empfangenden Schwestergesellschaft als gewinnsteuerneutrale Kapitaleinlage.

b) Modifizierte Dreieckstheorie

In diesem Fall werden auf der Ebene der Muttergesellschaft kein Beteiligungsertrag und keine Kapitaleinlage angenommen. Folglich verändern sich weder die Gewinnsteuerwerte noch die Gestehungskosten der Beteiligungen der Schwestergesellschaften.

Forderungsverzichte von Tochter- oder Schwestergesellschaften, die dem Drittvergleich standhalten, gelten als steuerlich abzugsfähiger Aufwand. Forderungsverzichte von Tochter- oder Schwestergesellschaften, die auf den Beziehungen zum Gesellschafter beruhen, sind nicht geschäftsmässig begründet und werden zum ausgewiesenen Gewinn hinzugerechnet.

Leistungen an Tochter- oder Schwestergesellschaften, die auf Besserungsscheinen oder Sanierungsgenussscheinen beruhen und bei denen es sich nicht um die Rückzahlung der ursprünglichen Forderung handelt, unterliegen der Gewinnsteuer. Der Beteiligungsabzug kann nicht geltend gemacht werden. 


\section{Sanierungsfusion mit Tochter- und Schwestergesellschaften}

$\mathrm{Zu}$ unterscheiden sind bei der Sanierungsfusion jene mit einer Mutter- mit einer Tochtergesellschaft (Upstream-Merger) und jene zwischen Schwestergesellschaften.

\section{Upstream-Merger}

Entsteht beim Upstream-Merger ein Buchverlust als Differenz zwischen dem Aktivenüberschuss zu Buchwerten der übernommenen Tochtergesellschaft und dem höheren Buchwert der untergegangenen Beteiligungsrechte, kann dieser bei der Muttergesellschaft als unechter Fusionsverlust gemäss Art. 61 Abs. 5 DBG steuerlich nicht abgezogen werden. Dies gilt jedoch nur für unechte Fusionsverluste, wenn die stillen Reserven und der Goodwill der übernommenen Gesellschaft den Buchverlust kompensieren.

Echte Fusionsverluste dagegen haben ihre Ursache in einer Überbewertung der Beteiligung an der Tochtergesellschaft und können deshalb als steuerlich abzugsfähiger Aufwand verbucht werden.

Die übernehmende Muttergesellschaft kann bei der Berechnung des steuerbaren Reingewinns die noch nicht berücksichtigten Vorjahresverluste der übertragenen Tochtergesellschaft nach Art. 67 Abs. 1 DBG bzw. Art. 25 Abs. 2 StHG geltend machen. Die Übernahme der Verlustvorträge der Tochter- durch die Muttergesellschaft wird bei Vorliegen einer Steuerumgehung verweigert, namentlich dann, wenn:

a) sich die Tochtergesellschaft in einem liquidationsreifen Zustand befindet (BGE 2A.583/2003) bzw. im Zeitpunkt der Absorption bereits keine Geschäftstätigkeit mehr ausübte (StE 2004, B 72.15.2 Nr. 5);

b) der übertragene Betrieb der Tochtergesellschaft kurz nach der Fusion eingestellt wird (BGE 2A.583/2003).

Auf Seite der Gesellschafter ergeben sich keine Steuerfolgen, unabhängig davon, ob sich Beteiligungsrechte im Privatvermögen einer natürlichen oder im Geschäftsvermögen einer natürlichen oder juristischen Person befinden.

\section{Schwesternfusion}

Analog verhält es sich bei der Schwesternfusion. Die übernehmende Schwestergesellschaft kann die bei der Berechnung des steuerbaren Reingewinns noch nicht berücksichtigte Vorjahresverluste der übertragenden Schwestergesellschaft nach Art. 67 Abs. 1 DBG und Art. 25 Abs. 2 StHG grundsätzlich geltend machen. Eine Übernahme der Vorjahresverluste ist jedoch ausgeschlos- 
sen, wenn eine Steuerumgehung vorliegt, namentlich wenn die übertragende Schwestergesellschaft wirtschaftlich liquidiert oder in liquide Form gebracht worden ist oder wenn ein durch Fusion übertragener Betrieb kurz nach der Fusion eingestellt wird.

Auf Seite der Gesellschafter ist zu unterscheiden, ob sich die Beteiligungsrechte im Privatvermögen oder im Geschäftsvermögen befinden:

a) Beteiligungsrechte im Privatvermögen einer natürlichen Person

Übernimmt eine Schwestergesellschaft mit echter Unterbilanz durch Annexion die Aktiven und Passiven einer von einer Privatpersonen beherrschten Schwestergesellschaft mit Reserven und Gewinnvortrag, erlangt die Privatperson durch diese Sanierung dann einen geldwerten Vorteil nach Art. 20 Abs. 1 Bst. c DBG bzw. Art. 20 Abs. 1bis DBG, wenn und soweit durch die Fusion übrige Reserven, die nicht als Reserven aus Kapitaleinlagen qualifizieren, vernichtet werden. Gleiches gilt auch im umgekehrten Fall, soweit übrige Reserven untergehen. Eine echte Unterbilanz ist in dem Umfang gegeben, als die ausgewiesenen Verluste die offenen und stillen Reserven übersteigen. Die modifizierte Dreieckstheorie findet dabei keine Anwendung.

b) Beteiligungsrechte im Geschäftsvermögen einer natürlichen Person

Bilden die Beteiligungsrechte an den Schwestergesellschaften beim Beteiligten Geschäftsvermögen, realisiert er bei Anwendung des Buchwertprinzips keinen steuerbaren Beteiligungsertrag. Die Einkommenssteuerwerte der Beteiligungen werden addiert und bleiben gesamthaft betrachtet unverändert.

c) Beteiligungsrechte einer juristischen Person

Do. wenn die Beteiligungsrechte an den Schwestergesellschaften Bestandteil einer juristischen Person sind. Die Gestehungskosten und Gewinnsteuerwerte der Beteiligungen werden addiert und bleiben gesamthaft unverändert. Allfällige wieder eingebrachte Abschreibungen sind steuerbar.

\section{Literaturverzeichnis}

Die aufgeführten Werke bilden zum vorliegenden Werk eine Auswahl an weiterführender Literatur und dienen dessen Ergänzung.

Basler Kommentar zum Schweizerischen Privatrecht, Obligationenrecht I, Art. 1-529 OR, 7. A., Basel 2019. 
Basler Kommentar zum Schweizerischen Privatrecht, Obligationenrecht II, Art. 530-964 OR, 5. A., Basel 2016.

BENZ ROLF, Handelsrechtliche und steuerrechtliche Grundsätze ordnungsmässiger Bilanzierung, Diss. Zürich 2000.

BlumER KARL, Die kaufmännische Bilanz, 10. erweiterte und überarbeitete A., Zürich 1989.

BÖCKLI PETER, Der Rangrücktritt im Spannungsfeld von Schuld- und Aktienrecht, in: Festgabe Walter R. Schluep, Bern 1988, 339 ff.

BÖCKLI PeTER, Schweizer Aktienrecht, 4. A., Zürich 2009.

Boemle MaX/LuTz RALF, Der Jahresabschluss. Bilanz, Erfolgsrechnung, Geldflussrechnung, Anhang, 5. A., Zürich 2008.

Boemle MaX/Stolz CARSTEn, Unternehmungsfinanzierung, Band 1. Grundlagen und Kapitalbeschaffung, 14. A., Zürich 2010.

Boemle Max/Stolz Carsten, Unternehmungsfinanzierung, Band 2. Besondere Finanzierungsanlässe und Finanzanalyse, 14. A., Zürich 2012.

CARlen Franz/Gianini Franz/Riniker Anton, Finanzbuchhaltung 1, Praxis der Finanzbuchhaltung, 16. A., Zürich 2020.

FER Swiss GAAP FER, Fachempfehlungen zur Rechnungslegung, FER Stiftung für Fachempfehlungen zur Rechnungslegung, Zürich 2020.

GURTNER PETER, Die handelsrechtlich ausschüttbare nichtbetriebsnotwendige Substanz nach dem neuen Recht der indirekten Teilliquidation, ASA 76 (2008), $553 \mathrm{ff}$.

GuRTNER PETER, Inflation Accounting und Steuern, ST 1982, 2 ff. und 38 ff.

Gurtner PETER, Neue Rechnungslegung - Prinzipielle Massgeblichkeit oder eigenständige Steuerbilanz?, ASA 69 (2000/01), 63 ff.

HELBLING CARL, Bilanz- und Erfolgsanalyse, 10., nachgeführte Auflage, Bern und Stuttgart 1997.

HELbling CARL, Unternehmensbewertung und Steuern, 9. A., Düsseldorf 1998.

HONSELL HEINRICH, Schweizerisches Obligationenrecht, Besonderer Teil, 10. A., Bern 2017.

HÜTTCHE TOBIAS/MEIER-MAZZUCATO GIORGIO, Unternehmensbewertung von Schweizer KMU, Kommentierung der Fachmitteilung „Unternehmensbewertung von kleinen und mittleren Unternehmen (KMU)“ von EXPERTsuisse, Hrsg. EXPERTsuisse, Zürich 2018.

JunGo AlEXANDRa ET AL., Das schweizerische Zivilgesetzbuch, 14. A., Zürich 2015.

KÄFER KARL, Die Bilanz als Zukunftsrechnung, 3. A., Zürich 1976.

KÄFER KARL, Die Erfolgsrechnung, Zürich 1977.

KÄFER KARL, Kommentar zum Obligationenrecht, Band VIII, 2. Abteilung, Die kaufmännische Buchführung, Bern 1981.

KäLIN OLIVER, Die Sanierung der Aktiengesellschaft, Ein Rechtshandbuch für Verwaltungsräte, Zürich, 2016.

KlÖti-Weber Marianne/Siegrist Dave/Weber Dieter, Kommentar zum Aargauer Steuergesetz, 4. A., Muri-Bern 2015.

Kren KostKiewicz Jolanta et AL., Handkommentar zum Schweizerischen Obligationenrecht, 3., aktualisierte A., Zürich 2016. 
LANZ ROLF, Controlling in kleinen und mittleren Unternehmen, 3. A., Bern und Stuttgart 1992. Locher Peter, Kommentar zum DBG, I. Teil, Art. 1-48 DBG, 2. A., Therwil/Basel 2019. LOCHER Peter, Kommentar zum DBG, II. Teil, Art. 49-101 DBG, Therwil/Basel 2004. LOCHER Peter, Kommentar zum DBG, III. Teil, Art. 102-222 DBG, Therwil/Basel 2015. Luginbühl Tanja/Affolter Marino Anja, „Exit“ aus der Nachlassstundung nach erfolgreicher Sanierung - Erste Erkenntnisse zum neuen Art. 296a SchKG, SZW/RSDA 3/2019.

Meier-Hayoz Arthur/Forstmoser Peter/Sethe Rolf, Schweizerisches Gesellschaftsrecht, 12. A., Bern 2018.

Meier-Mazzucato Giorgio, Entgeltliche Unternehmensnachfolge von KMU mit Schwerpunkt steuerliche Aspekte, 1. A., Bern 2009.

Meier-Mazzucato Giorgio, Steuerliche Aspekte der Unternehmensnachfolge - einige wesentliche Themenbereiche, TREX 6/2009.

Meier-Mazzucato Giorgio, Aspekte der Unternehmensnachfolge und Unternehmensbewertung - Teil 1, TREX 3/2013.

Meier-Mazzucato Giorgio, Bestimmung der steuerlich abzugsfähigen Vermögensverwaltungskosten für Wertschriften, TREX 1/2015.

Meier-Mazzucato Giorgio, Erweiterte modifizierte Dreieckstheorie zur Verbesserung von Unternehmensnachfolgeprozessen mittels Spaltung, TREX 3/2015.

Meier-Mazzucato Giorgio/Montandon Marc, Finanzierung der entgeltlichen Unternehmensnachfolge - Optimierung der Steuern und der Finanzierung, TREX 3/2011.

Meier-Mazzucato Giorgio, Montandon Marc, Wert- bzw. Preisperspektiven und Paketzuund -abschläge bei der Unternehmensbewertung und bei Unternehmenstransaktionen, TREX 5/2011.

Meier-Mazzucato Giorgio/Montandon Marc, Vorbereitende Strukturierung von Unternehmen im Unternehmensnachfolgeprozess, TREX 3/2012.

Meier-Mazzucato Giorgio/Montandon Marc, Aspekte der Unternehmensnachfolge und Unternehmensbewertung - Teil 2, TREX 4/2013.

Meier-Mazzucato Giorgio/Montandon Marc, Aspekte der Unternehmensnachfolge und Unternehmensbewertung - Teil 3, TREX 6/2013.

Meier-Mazzucato Giorgio/Ricklin Daniela, Earn-Out mit Bewertungs- und Finanzierungsfunktion als probate Methode bei der Unternehmensnachfolge, TREX 5/2014.

Meier-Mazzucato Giorgio, Erweiterte modifizierte Dreieckstheorie zur Verbesserung von Unternehmensnachfolgeprozessen mittels Spaltung, TREX 3/2015.

Meier-Mazzucato Giorgio, Upstream-Merger - steuerrechtliche Aspekte zu echtem bzw. unechtem Fusionsgewinn und Fusionsverlust, TREX 5/2017.

Meier-Mazzucato Giorgio, Strässle Verena, Stille Reserven nach Rechnungslegungsrecht und Bilanzsteuerrecht im Vergleich, TREX 5/2018.

Meier-Mazzucato Giorgio/Tasini Giulia, Kapitalkosten bzw. Kapitalkostensätze unter Berücksichtigung steuerlicher Aspekte mit Accounting bzw. Fundamental Beta, TREX 2/ 2019.

Reich Markus, Die Realisation stiller Reserven im Bilanzsteuerrecht, Zürich 1983. 
Reich Markus, Steuerrechtliche Aspekte des Fusionsgesetzes, FStR 2001, 4 ff.

Reich Markus, Steuerrecht, 3. A., Zürich 2020.

Richner Felix/Frei Walter/Kaufmann Stefan/Meuter Hans Ulrich, Handkommentar zum DBG, 3., überarbeitete A., Bern 2016.

Richner Felix/Frei Walter/Kaufmann Stefan/Meuter Hans Ulrich, Kommentar zum harmonisierten Zürcher Steuergesetz, 3. A., Zürich 2013.

Rihm Thomas W., Nachrangige Schuldverpflichtungen, Diss. Zürich 1992.

Schweizer Handbuch der Wirtschaftsprüfung, 2 Bände, Buchführung und Rechnungslegung und Eingeschränkte Revision, Zürich 2014.

Sprecher Thomas/Sommer Christa, Sanierung nach Aktienrecht, Bestandesaufnahme und Handlungsbedarf, Der Schweizer Treuhänder 6-7/2014.

Sprecher Thomas/Inderkum Matthias, Vernehmlassungsvorlage: Aktienrechtliche Sanierung, Vorentwurf des Bundesrats zur Änderung des Aktienrechts, Der Schweizer Treuhänder 3/2015.

Thommen Jean-Paul, Betriebswirtschaft und Management, 10., überarbeitete und erweiterte A., Zürich 2016.

Vogel Alexander et al.Fusionsgesetz, 3. A., Zürich 2017.

Volkart Rudolf/Wagner Alexander F., Corporate Finance, Grundlagen von Finanzierung und Investition, 7., aktualisierte A., Zürich 2018.

Volkart Rudolf., Finanzmanagement, Beiträge zu Theorie und Praxis, 7. A., Band I und Band II, Zürich 1998.

Volkart Rudolf, Wertorientierte Steuerpolitik, 2. A., Zürich 2006.

Watter Rolf et al. (Hrsg.), Basler Kommentar, Fusionsgesetz, 2. A., Basel 2015.

Weilenmann Paul, Planungsrechnung in der Unternehmung, 8. A., Zürich 1994.

Zweifel Martin/Beusch Michael (Hrsg.), Kommentar zum Schweizerischen Steuerrecht, Bundesgesetz über die Harmonisierung der direkten Steuern der Kantone und Gemeinden (StHG), 3. Auflage, Basel 2017.

Zweifel Martin/Beusch Michael (Hrsg.), Kommentar zum Schweizerischen Steuerrecht, Bundesgesetz über die direkte Bundessteuer, 3. A., Basel 2017.

Zweifel Martin/Beusch Michael/Bauer-Balmelli Maja (Hrsg.), Kommentar zum Bundesgesetz über die Verrechnungssteuer, Zürich 2012.

\section{Materialien}

Die nachfolgend aufgeführten Materialien dienen als Grundlagen zu den Ausführungen.

- Aktienrecht (Obligationenrecht SR 220), Änderung vom 19. Juni 2020.

- Botschaft zur Änderung des Obligationenrechts (Aktienrecht) vom 23. November 2016, 16.077, BBl 2017399.

- EXPERTsuisse, Fachmitteilung „Unternehmensbewertung von kleinen und mittleren Unternehmen (KMU)“, Datum der ersten Veröffentlichung: 6. September 2018. 
- EXPERTsuisse, PH 10, Schweizer Prüfungshinweis 10: Berichterstattung zur Prüfung von besonderen Vorgängen (gilt für Prüfungsberichte zu besonderen Vorgängen, die ab 15. Dezember 2013 datiert werden).

- EXPERTsuisse, Schweizer Prüfungsstandard 290, Pflichten der gesetzlichen Revisionsstelle bei Kapitalverlust und Überschuldung (gilt für Mandate als gesetzliche Revisionsstelle für Geschäftsjahre, die am 1. Januar 2010 oder danach beginnen).

- EXPERTsuisse, Schweizer Standard zur Eingeschränkten Revision (SER), Ausgabe 2015.

- Schweizer Kontenrahmen KMU. 


\title{
Spurensuche - SchKG-Relevantes in der Buchhaltung
}

\author{
Sikander von Bhicknapahari
}

\section{Inhalt}

I. Die Einjahresfrist dauert im Schnitt drei Jahre $\quad 52$

1. Die Rechtsprechung in Sachen Rechtsverzögerung 52

2. Statistische Hinweise auf die andauernde Rechtsverzögerung 53

II. Verjährungsrisiko 54

1. Keine Pflicht des Konkursamtes $\quad 54$

2. Als Gläubiger aktiv werden 55

III. Ausgangspunkt Ordnungsmässigkeit 55

1. Ordnungsgemässe Buchführung $\quad 56$

2. Ordnungsgemässe Rechnungslegung 56

IV. Mögliche Kontrollen 57

1. Vorjahresvergleich 57

2. Verkauf von Anlagevermögen $\quad 58$

3. Korrekte Anzahl von Vollzeitstellen $\quad 58$

4. Immaterielle Aktiven 58

5. Leasingverträge 59

6. Interne Verrechnungen 59

7. Bewertungen 60

8. Der Anhang 60

9. Insichgeschäfte 61

V. Schlussfolgerung 61

Kommentare $\quad 62$

$\begin{array}{ll}\text { Anhang } & 62\end{array}$

Die Frist zur Anfechtung verjährt innert zwei Jahren nach der Konkurseröffnung. Die Abwicklung eines Konkurses dauert im Durchschnitt drei Jahre. Ein rechtzeitiger Blick in die Buchhaltung könnte zeigen, ob eine Verjährungsunterbrechung eingeleitet werden soll. 


\section{Die Einjahresfrist dauert im Schnitt drei Jahre}

\section{Die Rechtsprechung in Sachen Rechtsverzögerung}

Gemäss Art. 270 Abs. 2 SchKG sollte ein Konkursverfahren innert eines Jahres abgeschlossen sein. Die Realität zeigt jedoch ein anderes Bild. Die Nichteinhaltung einer Frist durch ein Konkursamt war bereits 1993 ein Thema, welches zu einem Bundesgerichtsurteil führte. Zu jenem Zeitpunkt sah der Art. 270 SchKG vor, dass ein Konkursverfahren innert sechs Monaten durchgeführt sein sollte. Die Beschwerde, die zum BGE 119 III 1 führte, wurde fast drei Jahre nach der Konkurseröffnung eingereicht. Das Bundesgericht kam zu Schluss, dass eine solche Rechtsverzögerung dazu führen könne, dass ein Kanton sich unter Umständen haftpflichtig mache. Das Bundesgericht erinnerte den Kanton Bern daran, „dass der Kanton seinen Bürgern gegenüber zur Gewährung einer ordnungsgemässen Rechtspflege - wozu auch das Betreibungs- und Konkurswesen gehört - verpflichtet ist. Es bleibt für den Kanton nicht ohne finanzielle Folgen, wenn er sich dieser Verpflichtung entzieht und für seine Versäumnisse haftbar gemacht wird “1. Grund für die Verzögerung waren die Sparmassnahmen des Kantons Bern, welche dazu führten, dass das Konkursamt nicht über das notwendige Personal verfügte.

17 Jahre später behandelte das Kantonsgericht Basel-Land einen Fall von Rechtsverzögerung, bei dem eine Beschwerde rund zwei Jahre nach der Konkurseröffnung eingereicht wurde. Der Art. 270 SchKG wurde inzwischen mit Wirkung ab dem 1.1.1997 auf eine Einjahresfrist geändert. Der Grund für die Erhöhung der Frist auf ein Jahr: „Nach geltendem Recht soll ein Konkursverfahren innert sechs Monaten durchgeführt sein. Die Praxis hat gezeigt, dass diese Frist in aller Regel nicht ausreicht. Deshalb wird sie auf ein Jahr verlängert.“2 Das Kantonsgericht führte dabei u.a. aus, dass dieses im Recht liegende Verfahren nicht so bevorzugt behandelt werden dürfe, dass noch ältere Verfahren länger liegen blieben. Dies wäre unvereinbar mit dem Gebot der rechtsgleichen Behandlung. Schlussendlich führte das Kantonsgericht mit Verweis auf den BGE 119 III 1 aus, dass die personelle Vernachlässigung des Betreibungs- und Konkurswesens durch den Kanton diesen unter Umständen haftpflichtig machen könnte. ${ }^{3}$

BGE 119 III 1 E 3.

2 Botschaft des Bundesrates vom 8. Mai 1991 über die Änderung des Bundesgesetzes über Schuldbetreibung und Konkurs (SchKG), BBl 1991 III 1 ff., 161.

3 Entscheid 20010 735/LIA der AB SchKG vom 13. Juli 2010. 


\section{Statistische Hinweise auf die andauernde Rechtsverzögerung}

Beim Bundesamt für Statistik ist eine Tabelle mit aktuellen Daten über Konkursverfahren abrufbar. ${ }^{4}$ Gemäss dieser wurden in der Zeit von 2010 bis und mit 2019 insgesamt 145'852 Konkursverfahren eröffnet und 131'625 Verfahren abgeschlossen. Der Pendenzenberg hat somit um rund 10\% der Verfahren zugenommen. Betrachtet man zudem, dass 20'222 Verfahren basierend auf Art. 731b OR eröffnet wurden, so sind es wohl mehr als 10\% der normalen Konkursverfahren, die liegengeblieben sind. Bei den Fällen, die auf Art. 731b OR basieren, kann von Amtes wegen bei einem Organisationsmangel die Gesellschaft via Konkursverfahren liquidiert werden. ${ }^{5}$ In der Gerichtspraxis werde eine Frist zwischen 30 bis zu 60 Tagen für die Einberufung einer Generalversammlung gewährt. ${ }^{6}$ Es ist deshalb anzunehmen, dass die bei Nichtbehebung des Organisationsmangels notwendige Abwicklung des Konkurses auch innert einer Frist von wenigen Monaten erfolgt. Diese aus der Statistik ablesbare Realität von länger als einem Jahr dauernden Konkursverfahren, zeigt sich auch in der Resolving Insolvency-Statistik der Weltbank. In der Schweiz dauert gemäss deren Einschätzung ein Konkursverfahren im Durchschnitt drei Jahre. ${ }^{7}$ Die Statistik der Weltbank basiert auf einem angenommenen Fall, der nicht genau ab dem Moment der Konkurseröffnung startet. ${ }^{8}$ Die Auswertung im Vergleich mit anderen Ländern zeigt jedoch, dass die Schweiz bei der Abwicklung von Insolvenzen viel Zeit verstreichen lässt. Auf einer Liste mit 170 Ländern, weisen 109 Länder weniger als drei Jahre Dauer aus. 21 Länder benötigen drei Jahre für die Abwicklung.

Die Weltbank erstellt unter Berücksichtigung der Dauer, der Kosten und der \% Konkursdividende ein Ranking. Die Schweiz belegt dort vor Kenya, Indien und China und hinter der Slowakischen Republik, Azerbaijan und Kosovo den Rang 49. Lediglich 17 Länder wickeln ein Konkursverfahren innert eines Jahres ab. Spitzenreiter ist Irland mit 0.4 Jahren bei Kosten von $9 \%$ im Vergleich zu den 4.5\% Kosten die in der Schweiz anfallen. Die OECD High Income Länder weisen einen Durchschnitt von 1.7 Jahren bei 9.3\% Kosten aus.

\footnotetext{
4 <https://www.bfs.admin.ch/bfs/de/home/statistiken/industrie-dienstleistungen/ unternehmen-beschaeftigte/unternehmensdemografie/konkurse.assetdetail.12207036.html>.

Art. 731b Abs. 1 Ziff. 3 OR.

OFK OR-MÜLLER, Art. 731b, N 6.

$<$ https://www.doingbusiness.org/en/data/exploretopics/resolving-insolvency>.

8 Die Annahmen sind unter $<$ https://www.doingbusiness.org/en/methodology/resolvinginsolvency> aufgelistet.
} 
Es scheint, als habe seit dem BGE 119 III 1 bisher noch keine Schadenersatzklage bei einem Kanton dazu geführt, dass in Sachen Rechtsverzögerung bei der Abwicklung eines Konkurses ein generelles Umdenken stattgefunden hat. Grund dafür könnte sein, dass man je nach Fall einen hypothetischen Schaden einklagen müsste. Das Risiko, neben dem bereits erlittenen Verlust aus dem Konkurs auch noch die möglichen Rechtskosten für das Verfahren tragen zu müssen, schreckt ab.

\section{Verjährungsrisiko}

\section{Keine Pflicht des Konkursamtes}

Mit Blick auf die über der gesetzlichen Vorgabe liegenden Dauer von Konkursverfahren ist ein Gläubiger deshalb gut beraten, selbst rechtzeitig dafür besorgt zu sein, dass er keinen Nachteil wegen einer möglichen Verjährung erleidet. Um festzustellen, ob ggf. eine Anfechtung im Sinne von Art. 285 ff. SchKG möglich wäre, ist ein Blick in die Buchhaltung der konkursiten Gesellschaft notwendig. Gemäss Art. 292 Abs. 1 Ziff. 2 SchKG verjährt das Anfechtungsrecht zwei Jahre nach der Konkurseröffnung. Bis zum Inkrafttreten des revidierten Art. 292 SchKG per 1. Januar 2014 stand im Gesetz, dass das Anfechtungsrecht innert zwei Jahren verwirke. Gemäss Botschaft habe das wohl auf einem gesetzgeberischen Versehen beruht. ${ }^{9}$ Wer also erst nach der Mitteilung, dass der Konkurs abgeschlossen sei und die Konkursdividende $\mathrm{XY}$ \% betrage, im Detail prüfen möchte, was noch zusätzlich zu holen sei (z.B. durch Abtretung von Rechtsansprüchen im Sinne von Art. 260 SchKG), wird beim aktuell durchschnittlich drei Jahre dauernden Konkursverfahren mit einer Anfechtung zu spät sein.

Die Gläubiger müssten sich somit darauf verlassen können, dass im Rahmen eines Konkursverfahrens geprüft wird, ob Anfechtungen im Sinne von Art. 286 bis 288 SchKG möglich sind und das Konkursamt rechtzeitig handelt. Dies setzt voraus, dass im Konkursamt das notwendige Fachwissen vorhanden ist, um bei der Durchsicht der Buchhaltung zu erkennen, ob ein solcher Fall vorliegt. Das Bundesgericht hat jedoch entschieden, die Art. 286 bis 288 SchKG seien nicht von Amtes wegen zu prüfen. ${ }^{10}$ Es liegt somit im Interesse der Gläubiger, selbst rechtzeitig dafür zu sorgen, dass keine Verjährung eintritt.

\footnotetext{
9 Botschaft des Bundesrates vom 8. September 2010 zur Änderung des Bundesgesetzes über Schuldbetreibung und Konkurs (Sanierungsrecht), BBl 2010, 6455 ff., 6478. 


\section{Als Gläubiger aktiv werden}

In einem ersten Schritt ist zu prüfen, wie das Konkursamt reagiert, wenn es vor Ablauf der Zweijahresfrist konkret dazu aufgefordert würde, eine mögliche Verjährung zu unterbrechen. Es ist anzunehmen, dass ein solches Begehren abgelehnt wird, weil dem Konkursamt das Personal fehlt, um eine Buchhaltung im Detail auf mögliche Anfechtungsgründe zu durchleuchten. Zudem setzt es sich einem Haftungsrisiko aus, wenn es einen möglichen Anfechtungsgrund übersieht und keine verjährungsunterbrechende Handlung vornimmt.

Um eine mögliche Verjährung zu unterbrechen, wäre also ein Blick in die Geschäftsbücher der konkursiten Unternehmung durch den Gläubiger notwendig. Das heisst, das Konkursamt müsste ihm nicht nur die Unterlagen zur Verfügung stellen, sondern ggf. auch Einsicht in die Buchhaltung in digitaler Form ermöglichen. Mit Blick auf mögliche datenschutzrelevante Unterlagen (z.B. Lohnbuchhaltung mit Arztzeugnissen), müsste von Seiten des Konkursamtes vorher eine Triage der Belege vorgenommen werden.

\section{Ausgangspunkt Ordnungsmässigkeit}

Bei einer Durchsicht der Geschäftsbücher müsste ein detailliertes Inventar mit den dem Konkursamt übergebenen Buchhaltungsunterlagen vorliegen. In der Praxis liegt jedoch zumeist ein einfacher Lieferschein vor, in dem summarisch die Buchhaltung für das Jahr XY und einige Ordner aufgeführt werden. Ob unter dem Begriff Buchhaltung alle Konti, Journale, Saldolisten oder Hilfsbuchhaltungen im Sinne von Art. $1 \mathrm{GeBüV}$ gemeint sind, wird nicht weiter präzisiert. Ebenso ist nicht klar, ob die Buchhaltung auch in digitaler Form, z.B. in einem PDF-Format, vorliegt. Falls die Buchhaltung online geführt wurde, müssten Passwörter und Zugriffslinks vorhanden sein. Zudem muss sichergestellt sein, dass die Daten nicht vor Ablauf der Aufbewahrungsfrist gelöscht werden, d.h. es müssten die Abo-Gebühren bezahlt werden oder ein Backup in einem lesbaren Format vorliegen. ${ }^{11}$

Ohne detaillierte Aufstellung über die dem Konkursamt übergebenen Unterlagen, werden sich das Konkursamt und die verantwortliche Person bei der konkursiten Unternehmung gegenseitig die Verantwortung für einen fehlenden Beleg zuschieben.

1110 Jahre ab Ablauf des Geschäftsjahres gem. Art. 958 f Abs. 1 OR, zusätzliche Bestimmungen in Art. 747 OR bei Liquidation, siehe auch BGE 131 IV 56. 


\section{Ordnungsgemässe Buchführung}

Je professioneller eine Buchhaltung geführt wurde, desto leichter lässt sich feststellen, ob alles mit rechten Dingen zu und her ging. Das Gesetz verweist verschiedentlich darauf hin, dass die Buchführung nach Art und Umfang einer Unternehmung organisiert sein muss. ${ }^{12}$ Bei vielen - vor allem kleineren Unternehmen, wird der Dokumentation darüber, wie die Buchhaltung organisiert ist, wenig Beachtung geschenkt. Ohne Arbeitsanweisungen und Aufzeichnungen über die Organisation lässt sich für einen Aussenstehenden jedoch nicht immer nachvollziehen, basierend worauf ein Geschäftsvorfall erfasst wurde. ${ }^{13}$ Mit zu den Arbeitsanweisungen können auch Abläufe gehören, die im Sinne eines IKS eine korrekte Verbuchung sicherstellen sollten. ${ }^{14}$ In einer professionellen Buchhaltungssoftware führt das System ein Journal, welches den gesetzlichen Anforderungen entspricht. ${ }^{15}$ Nachträgliche, unter Umständen rückdatierte, oder geänderte Buchungen, würden bereits bei einer ersten Durchsicht des Journals auffallen. Aufgrund des Journals wäre auch ersichtlich, ob die Buchhaltung innerhalb der gesetzlichen Frist abgeschlossen wurde. ${ }^{16}$ Zeigt es sich, dass weder ein sauberer Arbeitsablauf gewährleistet war, noch ein Journal vorliegt aus dem die Chronologie inklusive aller Änderungen oder Löschungen ersichtlich ist, so dürfte dies ein erster Hinweis sein, die Buchhaltung genauer anzuschauen.

\section{Ordnungsgemässe Rechnungslegung}

Bei Unternehmen, die der Grösse wegen auf eine Revision verzichten (sog. Opting out, Art. 727a Abs. 2 OR), zeigt ein Blick in die Jahresrechnung, ob trotzdem die Minimalanforderungen gemäss Art. 959 ff. OR eingehalten wurden. Weisen die Bilanz und die Erfolgsrechnung nicht die notwendigen Positionen in der richtigen Reihenfolge aus, wäre dies ein erster Hinweis auf eine mangelnde Kompetenz in Sachen Rechnungslegung. ${ }^{17}$ Ein sehr kurz gehaltener Anhang ohne Erläuterungen, kann als Hinweis auf eine mangelnde Kompetenz oder ein bewusstes Nichtoffenlegen von Informationen sein.

Art. 957a Abs. 2 Ziff. 4 OR „Art und Grösse“, Art. 1 und Art. 4 GeBüV ,je nach Art und Umfang“.

13 Art. 4 GeBüV.

14 veb.ch Praxiskommentar OR-vON BHICKNAPAHARI, Art. 957a, N 58-62.

Art. 957a Abs. 2 Ziff. 1 OR, Art. 1 Abs. 2 lit. b GeBüV und Art. 3 GeBüV, siehe auch veb.ch Praxiskommentar OR-vON BHICKNAPAHARI, Art. 957a, N 1 ff. und Art. 958f, N 42 ff.

Gemäss Art. 958 Abs. 3 OR innert sechs Monaten.

17

Bilanz Mindestreihenfolge gemäss Art. 959a Abs. 1 OR, Erfolgsrechnung Mindestreihenfolge gemäss Art. 959b Abs. 2 OR.
} 
Die Jahresrechnung muss vom obersten Leitungs- oder Verwaltungsorgan unterzeichnet werden, zusätzlich auch von der für die Rechnungslegung zuständigen Person. ${ }^{18}$ Unterzeichnet die gleiche Person bei beiden Funktionen, so ist dies ein Hinweis auf fehlende checks and balances. Es ist Aufgabe des Verwaltungsrates, für eine Finanzkontrolle besorgt zu sein. ${ }^{19}$ In der Botschaft zum Art. 716a OR wurde ausgeführt, dass die Finanzkontrolle auch die interne Kontrolle umfasst. ${ }^{20}$

\section{Mögliche Kontrollen}

Da der Fokus auf den zwei Jahren Verjährungsfrist gemäss Art. 292 SchKG liegt, sind es Handlungen gemäss Art. 286 bis 288 SchKG, die von Interesse sind. Da z.B. bei Schenkungen die angefochten werden, lediglich die objektiven Umstände massgebend sind, reicht die Buchhaltung um festzustellen, ob hier ein anfechtbarer Fall vorliegt. ${ }^{21}$

Nachfolgend ein paar Beispiele, auf was geachtet werden könnte.

\section{Vorjahresvergleich}

Wesentliche Abweichungen im Vergleich zum Vorjahr bei Forderungen oder Verbindlichkeiten müssen im Zweifelsfall im Detail betrachtet werden. Eine Reduktion einer Forderung kurz vor einem Konkurs kann genauso eine Begünstigung darstellen, wie eine nicht gerechtfertigte Rückzahlung einer Verbindlichkeit. Sicherheitshalber muss nicht nur die in der Bilanz ausgewiesene Position betrachtet werden, sondern die Saldoliste mit den Anfangs- und Endbeständen der einzelnen Konti. Dies, weil eine nicht gerechtfertigte Rückzahlung einer Schuld z.B. mit der Aufnahme eines neuen Kredites erfolgt sein kann. Wurden Darlehen zurückgeführt, so ist zu prüfen, ob dies basierend auf einem Darlehensvertrag und unter Einhaltung der Kündigungsfrist erfolgte. Im Verkehr mit Nahestehenden finden sich z.B. Geschäftsfahrzeuge, die privat genutzt wurden, welche kurz vor einem Konkurs ohne entsprechende Vertragsgrundlage in Verrechnung mit einem Darlehen auf die Privatperson übertragen werden.

\footnotetext{
18 Art. 958 Abs. 3 OR.

19 Art. 716a Abs. 1 Ziff. 3.

20 Botschaft des Bundesrates vom 23. Februar 1983 über die Revision des Aktienrechts, BBl 1983 II, $922 \mathrm{ff}$.

21 OFK SchKG-KREN KostKiEWICZ, Art. 286, N 1.
} 


\section{Verkauf von Anlagevermögen}

Wurde vor dem Konkurs Anlagevermögen verkauft, so ist darauf zu achten, ob dieses zum Buchwert verkauft wurde. Im Normalfall löst ein Verkauf von Anlagevermögen eine zusätzliche Abschreibung oder einen Gewinn aus dem Verkauf von Anlagevermögen aus. Ein Verkauf zum Buchwert kann ein Hinweis auf einen nicht marktkonformen Preis sein.

BGE 117 IV 23 zeigt eine Variante, wie Anlagevermögen zur Rückzahlung von Verbindlichkeiten verwendet wurde. In jenem Fall wurde der Geldfluss wie bei einer normalen Abwicklung durchgeführt. Das Anlagevermögen wurde verkauft, mit dem Geld wurde eine Verbindlichkeit zurückbezahlt. Auf den ersten Blick ein normaler Geschäftsvorgang. Wirtschaftlich betrachtet handelte es sich jedoch um einen Tausch kurz vor dem Konkurs. Um einen solchen Fall zu erkennen, muss eine Gesamtübersicht über die verschiedenen juristischen und natürlichen Personen, die in einen Geschäftsfall involviert sind, vorliegen. Der Buchungsbeleg allein reicht nicht.

\section{Korrekte Anzahl von Vollzeitstellen}

Bei zehn oder weniger Vollzeitstellen kann auf eine Revision verzichtet werden. ${ }^{22}$ Stellt sich heraus, dass die Unternehmung in Realität mehr als zehn Vollzeitstellen hatte, so ist zu prüfen, ob die Unternehmung früher Gewinne ausgeschüttet hatte. Die Gewinnverteilungs-Beschlüsse der Generalversammlung wären in diesem Fall nichtig, da kein Revisionsbericht vorlag. ${ }^{23}$ Eine Rückforderung der zu Unrecht ausbezahlten Dividende wäre zu prüfen.

\section{Immaterielle Aktiven}

Originäre immaterielle Aktiven wie z.B. der Wert eines Kundenstamms, dürfen in einem Abschluss nach OR nicht aktiviert werden. ${ }^{24}$ Wird im Rahmen eines Konkurses ein solcher Wert ohne Entgelt auf eine Nachfolgegesellschaft übertragen, so findet keine Ausbuchung eines Aktivums statt. Es kann deshalb auf der Suche nach möglichen Anfechtungspunkten nicht nur auf die Bilanz abgestellt werden. Auch Software- und andere Entwicklungen, die nicht bilanziert werden (können), könnten ohne Entgelt in einer Auffanggesellschaft eines Nahestehenden weiterbenutzt werden. Dies findet ohne eine Ausbuchung eines Aktivums in den Büchern der konkursiten Unternehmung statt. Es muss 
deshalb bei der Durchsicht der Buchhaltung auch die Tätigkeit des Unternehmens im Detail betrachtet werden. Erst dann wird ersichtlich, ob nichtbilanzierte Werte vorhanden waren und unentgeltlich auf Dritte übertragen wurden.

\section{Leasingverträge}

Ein Leasinggut ist nicht immer als Aktivum in der Bilanz ausgewiesen, z.B. ein geleastes Fahrzeug. Wird der Leasingvertrag kurz vor einem Konkurs auf eine Privatperson übertragen, besteht die Möglichkeit, dass ein Wert ohne Buchung übertragen wird. Insbesondere in Fällen, bei denen eine erste Leasingrate sehr hoch angesetzt wurde. Wurde diese Leasingrate im Aufwand verbucht, würde ohne detaillierten Einblick in die Buchhaltung nicht erkannt, dass ohne Buchung ein Wert übertragen wurde.

\section{Interne Verrechnungen}

Ist das konkursite Unternehmen Teil eines Firmenkonglomerates gewesen oder sind Organe oder Aktionäre an anderen Firmen beteiligt die einen Leistungsaustausch mit der in Konkurs befindlichen Unternehmung hatten, so sind die Preise, die für den Leistungsaustausch angewendet wurden von Interesse. Gemäss Art. 24 Abs. 2 MWSTG muss der Preis für einen Leistungsaustausch unter eng verbundenen Personen dem Wert entsprechen, wie er unter unabhängigen Dritten vereinbart würde. ${ }^{25}$ Losgelöst von der Vorschrift im MWSTG kann ganz allgemein gesagt werden, dass ein falscher Preis eine falsche Aussage in der Jahresrechnung nach sich zieht. Ein z.B. zu hoher oder zu tiefer Aufwand zeigt nicht die wirtschaftliche Lage, wie sie vom Rechnungslegungsrecht gefordert wird. ${ }^{26}$

Die OECD Richtlinie für Verrechnungspreise führt verschiedene Berechnungsvarianten auf, die bei einer konzerninternen Leistung angewendet werden

25 Im Urteil des Bundesverwaltungsrechts A-2304/2019 vom 20. April 2020 wird aufgezeigt, welche steuerlichen Konsequenzen ein Nichtverrechnen einer Leistung haben kann.

26 Art. 957a Abs. 1 und Art. 958 Abs. 1 OR. 
könnten. ${ }^{27}$ Je nach Fall wäre, wenn kein Drittvergleich möglich ist, eine Begründung notwendig, weshalb die eine oder die andere Berechnungsvariante gewählt wurde.

Verrechnungspreise können nicht nur bei einem Leistungsaustausch zwischen zwei Unternehmen angewendet werden. Bereits die Frage, wie hoch der Lohn eines Nahestehenden sein darf oder wo gar kein Lohn gerechtfertigt ist, kann mit einem Drittvergleich überprüft werden. ${ }^{28}$

\section{Bewertungen}

Werden die Geschäftsbücher im Detail angeschaut, findet auch eine Überprüfung der Bewertungen von z.B. nicht verrechneten Leistungen statt. Dies um festzustellen, ob gegebenenfalls ein Übertrag von solchen Leistungen unter Wert stattgefunden hat. Eine solche Prüfung kann aber auch aufzeigen, dass in der Vergangenheit Aktiven zu hoch bilanziert wurden und so eine SanierungsGV (Art. 725 Abs. 1 OR) verspätet einberufen oder eine Überschuldung (Art. 725 Abs. 2 OR) zu spät ausgewiesen wurde. Ein Grund dafür kann sein, dass weiterhin zu Fortführungswerten statt zu Veräusserungswerten bilanziert wurde. ${ }^{29}$ Auch die Bilanzierung zu Einstandspreisen statt mittels verlustfreier Bewertung festzustellen, dass eine Position tiefer bewertet werden müsste, kann eine Überbewertung nach sich ziehen. ${ }^{30}$ Es würden so zwar keine Positionen zur Anfechtung aufgezeigt werden können, eine Konkursverschleppung kann jedoch zu Verantwortlichkeitsansprüchen gegenüber den Organen führen.

\section{Der Anhang}

Eine Durchsicht der in den letzten Jahren im Anhang aufgeführten Informationen kann auch zu möglichen Forderungen führen. Falls die nun konkursite

27 <http://www.keepeek.com/Digital-Asset-Management/oecd/taxation/oecd-transferpricing-guidelines-for-multinational-enterprises-and-tax-administrations-2017 tpg-2017-en\#.Wciuy8irTtQ> Die verschiedenen Berechnungsmethoden werden in Chapter II S. 97 ff. erklärt. Im Vordergrund stehen die Fremdvergleichspreismethode (Comparable Uncontrolled Price), die Wiederverkaufspreismethode (Resale Minus) und die Kostenaufschlagsmethode (Cost Plus). ). Wann welche Berechnungsart sinnvoll ist, hängt vom Einzelfall ab. Die ESTV erachtet z.B. gemäss Kreisschreiben 4 vom 19. März 2004 „die ,cost-plus'-Methode für Finanzdienstleistungen und Management-Funktionen nicht oder nur in seltenen Ausnahmefällen als adäquat." Der Entscheid 6B_755/2012 zeigt, dass eine nichtgerechtfertigte Lohnzahlung an die Ehefrau auch strafrechtliche Konsequenzen haben kann. Art. 958a Abs. 2 OR. Art. 960c Abs. 1 OR. 
Unternehmung zu einer Zeit, als sie noch über freie Reserven verfügte eigene Aktien gekauft hatte, so finden sich die Angaben dazu im Anhang. Wurde ein zu hoher Preis für diese Aktien vereinbart, so wäre hier mittels Absichtsanfechtung auf fünf Jahre zurück eine Forderung möglich. ${ }^{31}$

Bei einer Durchsicht des Anhangs besteht ganz generell die Möglichkeit, auf eine falsche Berichterstattung zu stossen. Im Zusammenhang mit einem Konkurs stellt sich die Frage, ob in den Jahren zuvor stille Reserven aufgelöst wurden und darüber im Anhang korrekt informiert wurde. ${ }^{32}$ Zudem kann es je nach dem Grund, der zum Konkurs geführt hat, notwendig sein, im Anhang über ein wesentliches Ereignis nach dem Bilanzstichtag zu orientieren. ${ }^{33}$ Die fehlerhafte Berichterstattung führt zwar nicht zu einer Anfechtungsposition, kann aber zu einer Verantwortlichkeit der Organe führen.

\section{Insichgeschäfte}

Art. 718b OR verlangt, dass einem Insichgeschäft ab Fr. 1'000.- ein schriftlicher Vertrag zu Grunde liegen muss. In Unternehmen, bei denen der Hauptaktionär zugleich auch Verwaltungsrat und Geschäftsführer in Personalunion ist, wird häufig ein Darlehenskonto oder Kontokorrent-Konto Aktionär geführt, weil keine klare Trennung zwischen Geschäft und Privat gelebt wird.

Neben dem Buchungsbeleg für eine Transaktion zwischen dem Verwaltungsrat und der Unternehmung muss jedoch zusätzlich auch der schriftliche Vertrag vorliegen. Fehlt dieser, könnte sonst eine Transaktion gegebenenfalls als nichtig betrachtet werden und eine Forderung gegenüber dem Verwaltungsrat auslösen. $^{34}$

\section{Schlussfolgerung}

Statistisch gesehen besteht die Gefahr, dass wegen Verzögerungen bei einer Konkursabwicklung die Gläubiger erst sehr spät über das Ergebnis orientiert werden. Um eine Anfechtung vorzunehmen, muss sichergestellt sein, dass eine verjährungsunterbrechende Massnahme eingeleitet wird. Da das Konkursamt hier nicht aktiv werden muss, ist es Sache der Gläubiger, mittels rechtzeitiger Einsicht in die Geschäftsbücher sicherzustellen, dass - wo notwendig entsprechend gehandelt wird. Die Themenvielfalt, die dabei beachtet werden

\footnotetext{
31 Art. 288 SchKG.

32 Nettoauflösung von stillen Reserven, Art. 959c Abs. 1 Ziff. 3 OR.

33 Art. 959c Abs. 2 Ziff. 13 OR.

34 OFK OR-GLANZMANN, Art. 718b, N 6.
} 
muss, ist aus der beiliegenden MindMap ersichtlich. Die Kosten für diese Aufgabe, die eigentlich Sache der Konkursverwaltung im Interesse aller Gläubiger sein müsste, trägt der Gläubiger, der sich hierfür engagiert.

\section{Literaturverzeichnis}

OR-Kommentar, Schweizerisches Obligationenrecht, in: Kren Kostkiewicz Jolanta/Wolf Stephan/Amstutz Marc/Fankhauser Roland (Hrsg.), 3. A., Zürich 2016 (zit. OFK OR-BEARBEITER, Art. XX, N YY).

SchKG-Kommentar, Schuldbetreibungs- und Konkursgesetz mit weiteren Erlassen, in: Kren Kostkiewicz Jolanta (Hrsg.), 20. A., Zürich 2020 (zit. OFK SchKG-BEARBEITER, Art. XX, N YY).

Rechnungslegung nach Obligationenrecht, veb.ch Praxiskommentar, in: Pfaff Dieter et al. (Hrsg.), ,2. A., Zürich 2019 (zit. veb.ch Praxiskommentar-BEARBEITER, Art. XX, N YY).

\section{Anhang}

MindMap mit Themenübersicht 


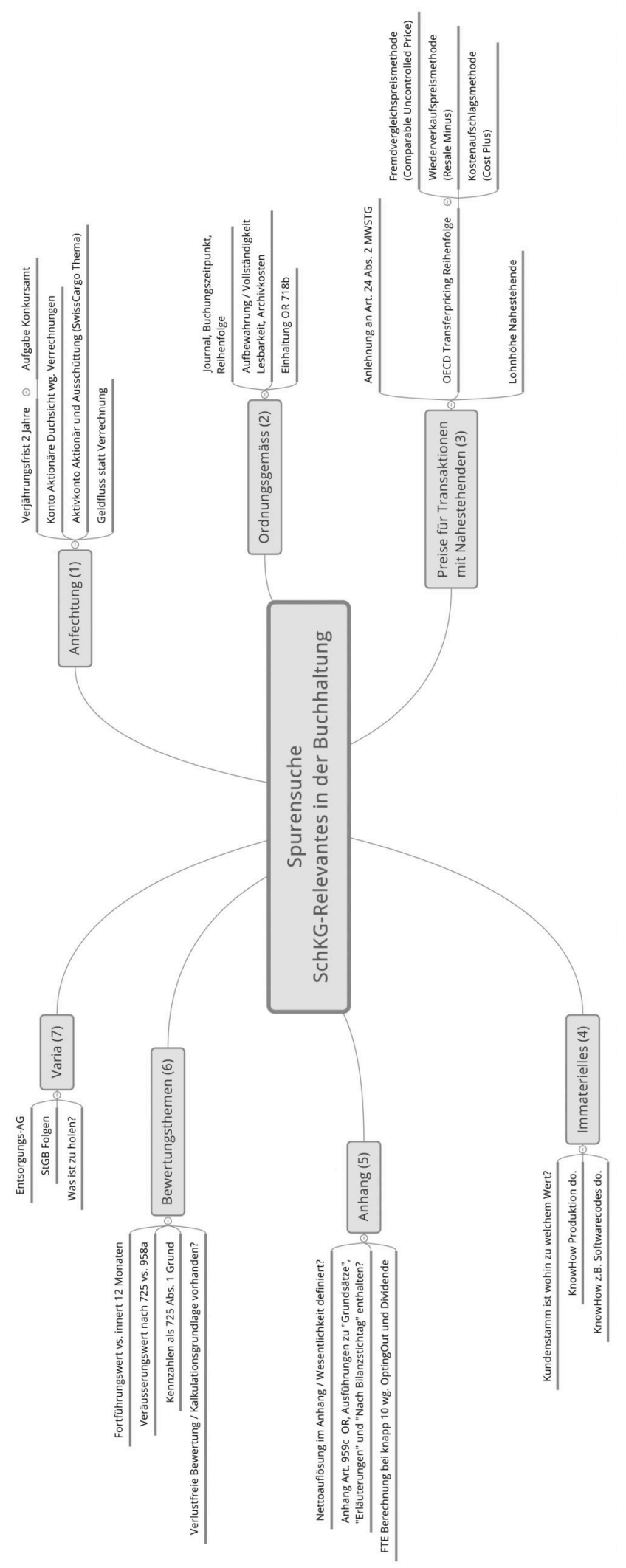





\section{Buchhalterische Darstellung von Sanierungsmassnahmen am praktischen Beispiel}

Oliver Kälin

\section{Inhalt}

I. Einleitung 66

\begin{tabular}{lr} 
II. Sachverhalt $\quad 67$ \\
\hline
\end{tabular}

III. COVID-19 69

IV. Standortbestimmung am 17. März 2020 70

1. Wann geht der Gesellschaft das Geld aus?

2. Hälftiger Kapitalverlust und Überschuldung $\quad 72$

3. Sofortmassnahmen: Löhne und Mietzins 76

a) Liquiditätssicherung $\quad 76$

b) Kurzarbeit $\quad 77$

V. Bilanz und Erfolgsrechnung am 31. März $2020 \quad 79$

VI. Ruhe vor dem Sturm im April $2020 \quad 81$

VII. Fortgang der Krise im Mai $2020 \quad 82$

1. Erneute Standortbestimmung und Einschätzung der Zukunft 82

2. Pro und contra des COVID-19-Kredits 85

3. Innenfinanzierung 85

a) Kapitalschnitt $\quad 85$

b) Ablösung der kreditgebenden Bank $\quad 86$

c) Kapitalzuschuss oder Sanierungsdarlehen $\quad 86$

d) Debt Equity Swap $\quad 87$

VIII. Einstweiliger Abschluss im Juni $2020 \quad 88$

1. Erfolgsrechnung, Bilanz und die Folgen $\quad 88$

2. Aussicht und Ergebnis $\quad 90$

\begin{tabular}{lr} 
Literatur- und Materialienverzeichnis & 90 \\
\hline
\end{tabular} 


\section{Einleitung}

Bereits der Römer Seneca soll es gesagt haben: Lang ist der Weg durch Lernen, kurz und wirkungsvoll durch Beispiele. ${ }^{1}$ Deshalb beschreibt dieser Aufsatz eine Fallstudie und im Mittelpunkt stehen Erfolgsrechnung und Bilanz. Sie zeigen, wie eine Aktiengesellschaft in eine finanzielle Krise rutscht und mit welchen Massnahmen der Verwaltungsrat dagegen ankämpft. Es geht um eine Gesellschaft, die Ende Januar 2020 in der Stadt Zürich ein Restaurant eröffnete und umgehend von Corona getroffen wurde.

Der vorliegende Aufsatz diente als Manuskript für den Vortrag an der Tagung des Europa Instituts vom 9. Juni 2020 zur Sanierung und Insolvenz von Unternehmen. Die Schweiz stand in der Coronakrise, Geschäfte und Restaurants waren aber wieder offen. Inzwischen - per Abgabedatum dieses Aufsatzes am 30. September 2020 - dauert die Coronakrise weiter an, eine zweite behördlich angeordnete Betriebsschliessung blieb bislang aus.

Wie fing alles an?

Im Februar 2020 erreichte das neue Coronavirus die Schweiz. Die Fallzahlen explodierten. Die Patienten standen Schlange in Spitälern und Arztpraxen. Der Bundesrat reagierte unverzüglich und verbot am 28. Februar 2020 öffentliche und private Veranstaltungen mit über 1000 Personen. ${ }^{2}$ Weil sich das Virus trotzdem weiter ausbreitete und immer mehr Menschen an der Lungenkrankheit COVID- $19^{3}$ litten, verstärkte der Bundesrat die Massnahmen und erliess zusätzliche Verordnungen, unter anderem die COVID-19-Verordnung 2 vom 13. März 2020. ${ }^{4}$ Gestützt auf diese Verordnung mussten öffentlich zugängliche Einrichtungen einstweilen bis am 19. April 2020 schliessen. ${ }^{5}$ Restaurants waren ebenfalls betroffen, ${ }^{6}$ auch unseres, welches im Januar 2020 erst eröffnet hatte.

1 Longum iter est praecepta, breve et efficax per exempla (Lucius Annaeus Seneca, 4 v.Chr. -65 n.Chr.).

2 Art. 2 der Verordnung über Massnahmen zur Bekämpfung des Coronavirus (COVID-19) vom 28. Februar 2020 (SR 818.101.24).

3 Coronavirus disease 2019, benannt durch die Weltgesundheitsorganisation (World Health Organization) WHO (siehe <https://www.who.int/emergencies/diseases/novel-coronavirus-2019>).

4 Verordnung 2 über Massnahmen zur Bekämpfung des Coronavirus (COVID-19) (COVID-19-Verordnung 2) vom 13. März 2020 (SR 818.101.24). Die vorausgehende Verordnung über Massnahmen zur Bekämpfung des Coronavirus (COVID-19) vom 28. Februar 2020 war befristet in Kraft bis am 15. März 2020 und wurde aufgehoben (Art. 11 COVID-19-Verordnung 2).

5 Art. 6 Abs. 2, Art. 12 Abs. 6 COVID-19-Verordnung 2.

$6 \quad$ Art. 6 Abs. 2 lit. b COVID-19-Verordnung 2. 
Bevor der Sachverhalt dargestellt wird und wir mehr erfahren über den Betrieb, die Gesellschaft und ihre Aktionäre, ist Folgendes vorwegzuschicken: Die Entwicklung der Gesellschaft wird von Februar bis Juni 2020 anhand von Erfolgsrechnungen und Bilanzen aufgezeigt. Dies widerspricht insofern der Realität, dass kaum ein Unternehmen monatliche Zwischenabschlüsse erstellt, sei es aus Zeit- oder aus Kostengründen, auch wenn dies dem Verwaltungsrat die Finanzkontrolle natürlich erleichtern würde (Art. 716a Abs. 1 Ziff. 3 OR).

Zur besseren Übersicht werden Bilanzen und Erfolgsrechnungen vereinfacht dargestellt:

1. Das Beispiel geht pauschal von Personalaufwand aus (Art. 959b Abs. 2 Ziff. 4 OR), ohne nach Lohnkosten und Beiträgen an Sozialversicherungen und die Pensionskasse zu unterscheiden.

2. In Mietverträgen über Geschäftsräume wird in aller Regel vereinbart, dass der Mietzins zu Monatsbeginn bezahlt werden muss. ${ }^{7}$ Vorliegender Mietvertrag geht indes mit Art. 257c OR von der Fälligkeit am Monatsende aus. (Damit kann auf eine monatliche Abgrenzung verzichtet werden und das damit verbundene Bilden transitorischer Aktiven für vorausbezahlte Mieten entfällt.) ${ }^{8}$

3. Unberücksichtigt bleiben: Bankzinsen, Versicherungsprämien (obligatorische Unfallversicherung, ${ }^{9}$ Krankentaggeldversicherung, ${ }^{10}$ Sachversicherungen) sowie die staatlichen Abgaben für die Bewilligung und den Betrieb des Lokals.

\section{Sachverhalt}

Am 16. Januar 2020 gründeten drei befreundete Gastronomen eine Aktiengesellschaft für den Betrieb eines Restaurants. Als Standort wählten sie die Stadt Zürich und die Küche sollte die römische sein. Die drei Aktionäre verwirklichten einen Jugendtraum. Sie schätzten ihre Erfolgsaussichten als gut ein und wollten gegen die zahlreichen italienischen Lokale in der Stadt bestehen.

Das Aktienkapital der Gesellschaft betrug Fr. 120'000, war voll liberiert und aufgeteilt in 120 Aktien mit einem Nennwert von je Fr. 1'000. Jeder der drei Aktionäre hielt 40 Aktien. Die Aktionäre waren alle zugleich Mitglieder des

\footnotetext{
In Abweichung von Art. 257c OR (ZK OR-Higl/BÜHLMANN, Art. 257c, N 14).

8 Zur zeitlichen Abgrenzung und der Bildung transitorischer Aktiven etwa MEYER, 77 ff.; zur zeitlichen Abgrenzung auch BÖCKLI, Rechnungslegung, Rz. 135; HWP, 35 f.

9 Art. 1a UVG; Art. 25 L-GAV.

10 Art. 23 L-GAV.
} 
Verwaltungsrats und führten den Betrieb selbst. Sie stellten vier Mitarbeiter an, zwei im Service und zwei in der Küche. Alle Arbeitnehmer waren vollzeitbeschäftigt.

Die Bilanz per 31. Januar 2020 sah folgendermassen aus:

Abb. 1

\begin{tabular}{|l|r|l|r|}
\hline Aktiven & CHF & Passiven & CHF \\
\hline Umlaufvermögen & & Fremdkapital & \\
\hline Flüssige Mittel & $80^{\prime} 000$ & Darlehen Bank & $70^{\prime} 000$ \\
\hline Vorräte & $600^{\prime} 000$ & & \\
\hline Anlagevermögen & & Eigenkapital & \\
\hline Mobiliar & 40 '000 & Aktienkapital & 120 '000 \\
\hline EDV & $10^{\prime} 000$ & & 190 '000 \\
\hline Total & $190^{\prime} 000$ & & \\
\hline
\end{tabular}

Das Restaurant feierte die Eröffnung am 1. Februar 2020 und der erste Monat verlief erfreulich. Die Erfolgsrechnung sah so aus:

Abb. 2

\begin{tabular}{|l|r|l|r|}
\hline Aufwand & CHF & Ertrag & CHF \\
\hline Warenaufwand & $15^{\prime} 000$ & Warenertrag & $65^{\prime} 000$ \\
\hline Personalaufwand & $35^{\prime} 000$ & & \\
\hline Miete & $10^{\prime} 000$ & & \\
\hline Gewinn & $5^{\prime} 000$ & & \\
\hline Total & $65^{\prime} 000$ & & $65^{\prime} 000$ \\
\hline
\end{tabular}

Die Erfolgsrechnung zeigt, dass die Gesellschaft im ersten Monat einen Gewinn von Fr. 5’000 erwirtschaftete. Dieser Gewinn (Art. 959a Abs. 2 Ziff. 3 lit. c OR) ${ }^{11}$ stellte zugleich den Bilanzgewinn ${ }^{12}$ am 29. Februar 2020 dar.

11 Art. 959a Abs. 2 Ziff. 3 lit. c OR spricht von der „gesetzlichen Gewinnreserve“ und bezeichnet damit erzielte, aber nicht ausgeschüttete Gewinne (MEYER, 38). Der Gewinn gehört zum Eigenkapital der Gesellschaft (Art. 959a Abs. 2 Ziff. 3 OR).

12 Zum Begriff BoemLe/LuTZ, 254; HANDSCHIN, Rz. 851. 
Die Vorräte (Lebensmittel) waren weiterhin Fr. 60'000 wert: Sie wurden im Umfang von Fr. 15'000 verbraucht, aber die Gesellschaft kaufte im selben Betrag neue Vorräte ein.

Die Bilanz per 29. Februar 2020 präsentierte sich wie folgt:

Abb. 3

\begin{tabular}{|l|r|l|r|}
\hline Aktiven & CHF & Passiven & CHF \\
\hline Umlaufvermögen & & Fremdkapital & \\
\hline Flüssige Mittel & $85^{\prime} 000$ & Darlehen Bank & $70^{\prime} 000$ \\
\hline Vorräte & 60 '000 & Eigenkapital & \\
\hline Anlagevermögen & & Aktienkapital & $120^{\prime} 000$ \\
\hline Mobiliar & 40 '000 & & \\
\hline EDV & $10^{\prime} 000$ & Bilanzgewinn & $5{ }^{\prime} 000$ \\
\hline Total & $195^{\prime} 000$ & & $195^{\prime} 000$ \\
\hline
\end{tabular}

\section{COVID-I9}

Unterdessen breitete sich die Krankheit COVID-19 in der Schweiz aus. Um die Übertragung zumindest zu verlangsamen, ordnete der Bundesrat am 16. März 2020 an, dass sämtliche Restaurationsbetriebe geschlossen werden. Die Schliessung sollte dauern vom 17. März 2020 bis einstweilen am 19. April 2020.

In aller Regel sind es mehrere Ursachen, die ein Unternehmen in eine Krise stürzen. ${ }^{13}$ Nicht so im vorliegenden Fall: Der Ausbruch des Coronavirus als Beispiel höherer Gewalt gilt als seltene Einzelursache, welche die Abwärtsspirale anstösst. In die Krise geschoben wurde die Gesellschaft durch die behördliche Betriebsschliessung - als Folge von Corona. Das plötzliche Schliessen des Restaurants war einschneidend und traf die Gesellschaft hart: Die Einnahmen brachen von heute (16. März 2020) auf morgen (17. März 2020) weg; die Fixkosten $^{14}$ hingegen, wie Miete und Löhne, waren weiterhin geschuldet.

13 ZK OR AG-HOMBURGER, Art. 725, N 1222; KÄLIN, Rz. 69 ff.

14 Fixkosten sind Kosten, die unabhängig vom Beschäftigungsgrad im Unternehmen anfallen und deshalb konstant sind (THOMMEN, 429; differenzierend SCHELLENBERG, 320). 


\section{Standortbestimmung am I7. März 2020}

Die fehlenden Einnahmen und die anhaltenden Kosten führten zu Verlusten in der Gesellschaft. Der Verwaltungsrat erkannte die Verluste als ein wesentliches Anzeichen einer Krise ${ }^{15}$ und kam am 17. März 2020 im leeren Lokal zusammen. Zwei Fragen galt es zu klären:

(1) Wann geht der Gesellschaft das Geld aus?

(2) Wann tritt der hälftige Kapitalverlust ein und wann die Überschuldung?

\section{Wann geht der Gesellschaft das Geld aus?}

Die Frage, wie lange eine Gesellschaft über genügend Geldreserven verfügt, ist anhand der Geldflussrechnung zu beantworten. Die Geldflussrechnung stellt die Veränderung der flüssigen Mittel dar und unterscheidet Ein- und Auszahlungen aus drei Quellen: Geschäftstätigkeit, Investitionstätigkeit und Finanzierungstätigkeit. ${ }^{16}$ Die Geldflussrechnung ist ein wichtiges Instrument zur Finanzplanung; die Finanzplanung wiederum gehört zu den unentziehbaren und unübertragbaren Aufgaben des Verwaltungsrats (Art. 716a Abs. 1 Ziff. 3 OR). ${ }^{17}$

Obligatorisch ist eine Geldflussrechnung indessen nur für grössere Unternehmen (Art. 961 Ziff. 2 OR). Grössere Unternehmen sind solche, die von Gesetzes wegen eine ordentliche Revision durchführen müssen. ${ }^{18}$ Welche das sind, sagt Art. $727 \mathrm{OR}^{19}$

\section{KÄLIN, Rz. 39 ff.}

16 Botschaft des Bundesrates vom 21. Dezember 2007 zur Änderung des Obligationenrechts (Aktienrecht und Rechnungslegungsrecht sowie Anpassungen im Recht der Kollektiv- und der Kommanditgesellschaft, im GmbH-Recht, Genossenschafts-, Handelsregister- sowie Firmenrecht) , BBl 2008, 1589 ff., 1717. Das Gesetz äussert sich nicht dazu, wie die Geldflussrechnung zu gliedern ist, die Gliederung sollte sich indessen an der Mindestgliederung der Bilanz (Art. 959a OR) orientieren (HWP, 277).

17 BöckLI, Aktienrecht, § 13 Rz. 353; ZK OR AG-HOMBURGER, Art. 716a, N 563.

18 Botschaft Aktienrecht/Rechnungslegungsrecht, BBl 20081716.

19 (a) Publikumsgesellschaften, (b) wirtschaftlich bedeutende Unternehmen (die zwei von drei Grössenkriterien in zwei aufeinander folgenden Geschäftsjahren überschreiten: Fr. 10 Millionen Bilanzsumme, Fr. 20 Millionen Umsatzerlös und 50 Vollzeitstellen im Jahresdurchschnitt [Art. 727 Ziff. 2 OR]) und (c) Unternehmen, die eine Konzernrechnung erstellen müssen. 
Die Gesellschaft in unserem Beispiel qualifiziert als KMU und muss von Gesetzes wegen keine Geldflussrechnung erstellen. Da die Verhältnisse übersichtlich sind, verzichtete der Verwaltungsrat auf eine freiwillige Mittelflussrechnung.

Regelmässig unverzichtbar zur Beurteilung der wirtschaftlichen Lage bleiben hingegen Bilanz und Erfolgsrechnung per Stichtag. Dies, obschon der Gesetzeswortlaut von Art. 725 Abs. 2 OR nur eine Zwischenbilanz verlangt (und nicht auch eine Zwischenerfolgsrechnung). ${ }^{20}$ Der Anhang kann weggelassen werden. ${ }^{21}$ Allerdings wird der Verwaltungsrat gewisse Punkte besprechen müssen, die in den Anhang gehören. Darunter fallen Eventualverbindlichkeiten (Art. 959c Abs. 2 Ziff. 10 OR), aussergewöhnliche Positionen in der Erfolgsrechnung (Art. 959c Abs. 2 Ziff. 12 OR) und wesentliche Ereignisse nach dem Stichtag (Art. 959c Abs. 2 Ziff. 13 OR).

Wird keine Geldflussrechnung erstellt, muss der Verwaltungsrat die Liquidität dennoch planen und sicherstellen, dass der Gesellschaft das Geld nicht ausgeht. Um zu berechnen, wie lange die verbleibenden flüssigen Mittel den Betrieb aufrecht erhalten, liefert die Cash Burn Rate einen wichtigen Anhaltspunkt.

Die Cash Burn Rate wird mit den Angaben aus Bilanz und Erfolgsrechnung anhand folgender Formel berechnet: ${ }^{22}$

$$
\frac{\text { Flüssige Mittel }+ \text { kurzfristige Forderungen }}{\text { liquiditätswirksame Aufwendungen }} \times 365 \text { Tage }
$$

Weil die Restaurant-Gesellschaft nur im Februar 2020 aktiv war und folglich nur die Finanzzahlen eines Monats vorliegen, sind die Aufwendungen zu verzwölffachen.

Vorräte für den März 2020 kaufte die Gesellschaft bereits im Februar ein (Fr. 15'000). Während der Betriebsschliessung entstand deshalb kein weiterer Warenaufwand. Das führt zu folgender Rechnung:

20 Botschaft des Bundesrates vom 23. Februar 1983 über die Revision des Aktienrechts, BBl 1983 II, 745 ff., 927; siehe BöCKLI, Aktienrecht, § 13 Rz. 770a, mit zutreffendem Hinweis auf die Notwendigkeit einer simplifizierten Zwischenerfolgsrechnung.

21 BöckLI, Aktienrecht, § 13 Rz. 770a, empfiehlt einen Anhang, wenn auch stark vereinfacht.

22 Nach MEYER, 147. 


$$
\frac{F r .85^{\prime} 000}{12 \times\left(F r .35^{\prime} 000+F r .10^{\prime} 000\right)} \times 365 \text { Tage }
$$

Das Resultat ergibt 57.45. Die Zahl besagt, dass die Gesellschaft das Restaurant für 57 Tage, also knapp zwei Monate, geschlossen halten kann, bevor das Geld ausgeht.

Dieses Ergebnis ist insofern zu korrigieren, als verschiedene Schulden unterschiedliche Fälligkeiten aufweisen: Warenlieferungen müssen in der Regel innert 30 Tagen bezahlt werden, die Löhne sind spätestens am Monatsende auszurichten (Art. 323 Abs. 1 OR). Zudem überlebt auch eine Gesellschaft ohne Liquidität eine gewisse Zeit, da die wenigsten Gläubiger umgehend betreiben und das Betreibungsverfahren ebenfalls Zeit in Anspruch nimmt. Die Formel zur Cash Burn Rate kann die verbleibende Anzahl Tage somit nicht exakt berechnen und bleibt ungenau. Ihr Erkenntniswert allerdings ist nicht zu unterschätzen: Der Verwaltungsrat erhält umgehend die Antwort darauf, ob das Geld bereits in einem Monat ausgeht oder ob der Betrieb noch wenige Wochen weiterbestehen kann.

Der Verwaltungsrat in unserem Beispiel überlegt Folgendes: Die Gesellschaft übersteht die behördliche Schliessung ohne liquiditätssichernde Massnahmen kaum. Die Zeitspanne vom 17. März bis am 19. April 2020 ist zu lang. Zudem muss die Gesellschaft für eine Wiedereröffnung des Restaurants sämtliche Vorräte neu einkaufen, wozu Liquidität nötig ist. Entsprechend verkürzt sich die Anzahl Tage.

Nach diesen Überlegungen kam der Verwaltungsrat zum Schluss, dass die Gesellschaft mit der vorhandenen Liquidität einen geschlossenen Betrieb einen Monat überlebt, zwei hingegen nicht.

\section{Hälftiger Kapitalverlust und Überschuldung}

Art. 725 OR sieht zwei Schwellen vor: Den hälftigen Kapitalverlust und die Überschuldung. Beides sind Bilanzgrössen.

Den hälftigen Kapitalverlust erleidet eine Gesellschaft, wenn das Eigenkapital kleiner ist als die Hälfte des Aktienkapitals und der gesetzlichen Reserven (Art. 725 Abs. 1 OR). ${ }^{23}$ Die Aktiven werden nach Art. 958a Abs. 1 OR zu Fortführungswerten eingesetzt. ${ }^{24}$ 
Nach dem hälftigen Kapitalverlust tritt die Überschuldung ein, wenn der Wert der Aktiven sowohl zu Fortführungs- wie auch zu Veräusserungswerten tiefer liegt als der Wert der Forderungen der Gesellschaftsgläubiger (Art. 725 Abs. 2 OR). ${ }^{25}$

In unserem Beispiel erreicht die Gesellschaft den hälftigen Kapitalverlust, sobald das Eigenkapital Fr. 60'000 unterschreitet. ${ }^{26}$ Die Bewertung der Aktiven ist stets schwierig und hat einen, wenn nicht den wesentlichen Einfluss auf das Finanzbild eines Unternehmens. ${ }^{27}$

Keine Probleme verursachen die flüssigen Mittel; sie werden zum Nennwert eingesetzt. ${ }^{28}$ Bereits aber die Bewertung der Vorräte wird anspruchsvoller: Die Gesellschaft hält Lebensmittel für Fr. 60'000 (vorne Abb. 1). Fr. 60'000 entsprechen dem Anschaffungswert (Art. 960a Abs. 1 OR). Zu den Vorräten (Art. 959a Abs. 1 Ziff. 1 lit. d und Art. 960c OR) gehören sowohl Rohmaterialien wie auch unfertige Erzeugnisse (Art. 960c Abs. 2 OR) und damit sämtliche Werkstoffe, die zur Herstellung fertiger Produkte verwendet werden. ${ }^{29}$ In einem Restaurant zählen die eingekauften Lebensmittel dazu sowie Zwischenprodukte, z.B. Teig oder die Basis für Saucen.

Die COVID-19-Verordnung 2 trat am 13. März 2020 in Kraft und der Bundesrat verordnete mit Ergänzung vom 16. März 2020 die Schliessung sämtlicher Restaurationsbetriebe bis am 19. April 2020. ${ }^{30}$ Am 17. März 2020 schloss das Restaurant also über Nacht.

Diejenigen Lebensmittel, die nicht haltbar gemacht werden konnten (z.B. durch Einfrieren oder Vakuumieren), waren entweder zu verbrauchen oder wegzuwerfen. Die Vorräte waren folglich nicht mehr Fr. 60'000 wert und der Wert musste berichtigt werden. Was den Zeitpunkt angeht, so ist eine Wertberichtigung bei Eintritt der Werteinbusse zu verbuchen. ${ }^{31}$ Entsprechend war die Bilanzposition Vorräte bei Stillegung des Restaurants nach Art. 960 Abs. 3

25 BBl 1983 II, 927 („Benachrichtigung des Richters bei Überschuldung zu Fortführungs- und Veräusserungswerten“).

26 Das AK beträgt Fr. 120'000. Reserven bildete die neu gegründete Gesellschaft noch keine.

27 „Die Frage der Bewertung gehört damit zu den zentralen Problemstellungen des Rechnungswesens." (MEYER, 86).

28 HWP, 127.

29 BÖCKLI, Rechnungslegung, Rz. 896; HÜTTCHE, veb.ch Praxiskommentar, Art. 960c, N 6.

30 Art. 12 Abs. 6 COVID-19-Verordnung 2.

31 „Bestehen konkrete Anzeichen für eine Überbewertung von Aktiven (...), so sind die Werte zu überprüfen und gegebenenfalls anzupassen." (Art. 960 Abs. 3 OR); siehe HWP, 203; BÖCKLI, Rechnungslegung, Rz. 986; HANDSCHIN, Rz. 718. 
OR im Wert zu berichtigen. Der Buchwert eines Aktivums wird herabgesetzt als Reaktion auf eine aussergewöhnliche, meist nicht vorausgesehene Wertbeeinträchtigung. ${ }^{32}$

Der Verwaltungsrat bewertete die Vorräte per 17. März 2020 neu und entschied, den Betrieb kurzfristig als Take-away weiterzuführen. Damit - so die Schätzung - können die Vorräte bis Ende März für Fr. 10'000 verkauft werden.

Die Bilanzposition wies daher am 17. März 2020 einen berichtigten Wert von Fr. 10'000 auf. Die Abschreibung von Fr. 50'000 stellte einen Verlust dar, der in der Erfolgsrechnung März zu verbuchen war.

Weiter waren in der Erfolgsrechnung die Löhne (Fr. 35'000) und die Miete (Fr. 10'000) zu erfassen. Zudem hatte die Gesellschaft anfangs März für Fr. 5'000 Waren eingekauft. Bis am 16. März 2020 (vor der Schliessung) erwirtschaftete der Betrieb einen Umsatz von Fr. 25’000. Somit präsentierte sich die Erfolgsrechnung für März 2020 folgendermassen:

Abb. 4

\begin{tabular}{|l|r|l|r|}
\hline Aufwand & CHF & Ertrag & CHF \\
\hline Warenaufwand $^{33}$ & $55^{\prime} 000$ & Warenertrag & $25^{\prime} 000$ \\
\hline Personalaufwand & $35^{\prime} 000$ & & \\
\hline Miete & $10^{\prime} 000$ & Verlust & $75^{\prime} 000$ \\
\hline Total & $100^{\prime} 000$ & & $100^{\prime} 000$ \\
\hline
\end{tabular}

Durch den gegenüber dem Februar kleinen Ertrag und der Sonderabschreibung auf den Vorräten erlitt die Gesellschaft im März einen Verlust von Fr. 75'000. Liquiditätswirksam flossen Fr. 25'000 ab; ${ }^{34}$ zudem reduzierte sich das Gesellschaftsvermögen aufgrund der Abschreibung um Fr. 50'000.

Die Planbilanz per 31. März 2020 sah nach diesen Bewegungen wie folgt aus:

32 BöCKLI, Rechnungslegung, Rz. 983; MÜLLER/HENRY/BARMETTLER, veb.ch Praxiskommentar, Art. 960, N 28.

33 Fr. 5'000 (Warenaufwand) + Fr. 50'000 (Wertberichtigung Vorräte).

34 Fr. 25'000 (Warenertrag) - Fr. 5'000 (Warenaufwand) - Fr. 35'000 (Personalaufwand) Fr. 10'000 (Mietzins). 
Abb. 5

\begin{tabular}{|l|r|l|r|}
\hline Aktiven & CHF & Passiven & CHF \\
\hline Umlaufvermögen & & Fremdkapital & \\
\hline Flüssige Mittel $^{35}$ & $60^{\prime} 000$ & Darlehen Bank & $70^{\prime} 000$ \\
\hline Vorräte & $10^{\prime} 000$ & & \\
\hline Anlagevermögen & & Eigenkapital & \\
\hline Mobiliar & 40 '000 & Aktienkapital & 120 '000 \\
\hline EDV & $10^{\prime} 000$ & Bilanzverlust & $-70^{\prime} 000$ \\
\hline Total & 120 '000 & & 120 '000 \\
\hline
\end{tabular}

Durch den Verlust im März schmolz der Februar-Gewinn (Fr. 5'000) weg. Die Gesellschaft wies einen Bilanzverlust (oder: Verlustvortrag) von Fr. 70'000 aus.

Die Bilanz per 31. März 2020 brachte drei Erkenntnisse:

(1) Sie bestätigte die Berechnung der Formel zur Cash Burn Rate: Erzielt die Gesellschaft im April und im Mai aufgrund der Betriebsschliessung keinen Umsatz, kann sie die Fixkosten im April zwar noch decken (Fr. 45’000), im Mai hingegen nicht mehr.

(2) Der hälftige Kapitalverlust trat ein: Das Eigenkapital beträgt neu Fr. $50^{\prime} 000^{36}$ und liegt damit unter dem Wert des hälftigen Aktienkapitals (Fr. 60'000). Anders formuliert überragt der Bilanzverlust von Fr. 70'000 die Hälfte des Aktienkapitals von Fr. 60'000.

Bildlich gesprochen frisst sich der Verlust im März ins Eigenkapital der Gesellschaft hinein. Zutreffend spricht BöckLI von der Pufferfunktion des Eigenkapitals. ${ }^{37}$ So auch in unserem Fall: Das Eigenkapital absorbierte den Verlust und fing die drohende Überschuldung auf.

(3) Ist das Eigenkapital aufgebraucht, tritt die Überschuldung ein. Diese drohte im Folgemonat April: Bleiben die Fixkosten unverändert, würden im April wieder Fr. 45'000 abfliessen. Das Eigenkapital beträgt dann nur noch Fr. 5'000 und zieht die Gesellschaft in die Überschuldung.

35 Fr. 85'000 (per 29. Februar 2020) + Fr. 25'000 (Ertrag) - Fr. 5'000 (Aufwand für neue Vorräte) - Fr. 35'000 (Personal) - Fr. 10'000 (Miete).

36 Aktiven (Fr. 120'000) minus Schulden (Fr. 70'000) = Aktienkapital (Fr. 120'000) minus Bilanzverlust (Fr. 70'000) $=$ Fr. 50'000.

37 BöCKLI, Rechnungslegung, Rz. 312; siehe HANDSCHIN, Rz. 388. 


\section{Sofortmassnahmen: Löhne und Mietzins}

\section{a) Liquiditätssicherung}

Art. 725 Abs. 1 OR verpflichtet den Verwaltungsrat, bei hälftigem Kapitalverlust eine ausserordentliche Generalversammlung einzuberufen, die sogenannte Sanierungsversammlung. ${ }^{38}$ Unsere drei Verwaltungsratsmitglieder waren sich dessen bewusst und kamen umgehend wieder zusammen. Da sie zugleich sämtliche Aktionäre waren, gilt jede Verwaltungsratssitzung als Universalversammlung nach Art. 701 OR.

Die drei Verwaltungsratsmitglieder/Aktionäre besprachen die Lage. Sie waren sich einig, dass der Untergang drohte und Sparmassnahmen nötig waren. ${ }^{39} \mathrm{Mit}$ Sparmassnahmen galt es, die Fixkosten zu senken und die Liquidität zu schonen. Fixkostenpositionen bestanden zwei: Die Löhne und der Mietzins.

Die Gesellschaft beschäftigte sieben Angestellte, die drei Aktionäre und vier Mitarbeiter. Alle sieben Arbeitsverträge lauteten gleich, waren knapp gefasst und verwiesen auf das OR: Sie galten unbefristet, Probezeit war keine vereinbart. ${ }^{40}$ Die Kündigungsfrist der jungen Arbeitsverhältnisse dauerte einen Monat (Art. 335c Abs. 1 OR). Die Personalkosten betrugen Fr. 5'000 pro Mitarbeiter pro Monat.

Der Verwaltungsrat überlegte, sich im März keinen Lohn auszuzahlen. Zudem baten die Mitglieder den Vermieter um Unterstützung. Dieser erklärte sich bereit, der Gesellschaft den Mietzins für März, April und Mail einstweilen zu stunden.

Die zurückbehaltenen Löhne und die gestundeten Mietzinse kamen der Gesellschaft als Liquidität zugute. Allerdings stellten sie eine Schuld dar und waren als Fremdkapital zu verbuchen. Die Erfolgsrechnung blieb entsprechend unverändert (Abb. 4).

Die Verwaltungsratsmitglieder erstellten die Planbilanz per 31. März 2020 neu:

BBl 1983 II, 927; BÖCKLI, Rechnungslegungsrecht, Rz. 454; KÄLIN, Rz. 443 ff.

Siehe KÄLIN, Rz. 137 ff., Rz. 154 ff.

40

Was zulässig ist (Art. 335b Abs. 2 OR; siehe Urteil des Bundesgerichts 4C.278/2003 vom

5. November 2003 E. 2.3). 
Abb. 6

\begin{tabular}{|l|r|l|r|}
\hline Aktiven & CHF & Passiven & CHF \\
\hline Umlaufvermögen & & Fremdkapital & \\
\hline Flüssige Mittel & $85^{\prime} 000$ & Darlehen Bank & $70^{\prime} 000$ \\
\hline Vorräte & $10^{\prime} 000$ & Darlehen Aktionäre & $15^{\prime} 000$ \\
\hline Anlagevermögen & & Darlehen Vermieter ${ }^{42}$ & $10^{\prime} 000$ \\
\hline Mobiliar & $40^{\prime} 000$ & Eigenkapital & \\
\hline EDV & $10^{\prime} 000$ & Aktienkapital & $120^{\prime} 000$ \\
\hline & & Bilanzverlust & $-70^{\prime} 000$ \\
\hline Total & $145^{\prime} 000$ & & $145^{\prime} 000$ \\
\hline
\end{tabular}

Die Stundungen der drei Löhne und des Mietzinses erhöhten zwar die Liquidität um Fr. 25'000 und die flüssigen Mittel stiegen von Fr. 60'000 auf Fr. 85’000. Der Bilanzverlust blieb indessen gleich. Die Stundungen bewirkten keine Abnahme der Schulden und der hälftige Kapitalverlust bestand nach wie vor. Das Gesellschaftsvermögen verringerte sich und die Überschuldung drohte. Die Gesellschaft glitt weiterhin dem Konkurs entgegen und weder Mietzinsstundung noch Lohnstundungen halfen dagegen.

\section{b) Kurzarbeit}

Ende März beschloss der Bundesrat ein Massnahmenpaket zur Abfederung der wirtschaftlichen Folgen. ${ }^{43}$ Der Zugang zur Kurzarbeit wurde ausgeweitet und vereinfacht. Die Karenzfrist fiel weg und Kurzarbeitsentschädigung konnte auch für arbeitgeberähnliche Angestellte ausgerichtet werden. ${ }^{44}$ Somit profitierten die drei Aktionäre in unserem Beispiel. Ebenfalls erleichtert wurden die Auszahlungsmodalitäten. ${ }^{45}$

41 Aufgeschobene Auszahlung der März-Löhne.

42 Stundung der März-Miete.

43 Medienmitteilung des Bundesrats vom 20. März 2020 („Coronavirus: Massnahmenpaket zur Abfederung der wirtschaftlichen Folgen"), abrufbar unter <https://www.admin.ch/gov/ de/start/dokumentation/medienmitteilungen>.

44 Art. 2 und Art. 3 der Verordnung über Massnahmen im Bereich der Arbeitslosenversicherung im Zusammenhang mit dem Coronavirus (COVID-19-Verordnung Arbeitslosenversicherung) vom 20. März 2020 (SR 837.033).

45 Art. 6 f. COVID-19-Verordnung Arbeitslosenversicherung. 
Da Stundungen der Lohnforderungen und der Mietzinse kein Mittel gegen die drohende Überschuldung waren, beriet der Verwaltungsrat darüber, Kurzarbeitsentschädigung zu beantragen oder Mitarbeiter zu entlassen. Für die Kurzarbeit sprach, dass die Voraussetzungen gelockert wurden und die Gesellschaft umgehend staatliche Hilfe erhält; dagegen sprach, dass die Gesellschaft als Arbeitgeberin das Lohnrisiko nach Ablauf der Kurzarbeitsentschädigung wieder trägt. Der Verwaltungsrat konnte sich nur für das eine oder das andere entscheiden: Kurzarbeitsentschädigung setzt ein ungekündigtes Arbeitsverhältnis voraus (Art. 31 Abs. 1 lit. c $\mathrm{AVIG}^{46}$ ).

Der Verwaltungsrat besprach zunächst, dass die bundesrätlich verordnete Schliessung der verschiedenen Betriebe ${ }^{47}$ einstweilen bis am 19. April 2020 gilt. $^{48} \mathrm{Ob}$ die Schliessung verlängert, gelockert oder aufgehoben wird, war Ende März unklar. Der Verwaltungsrat nahm aber an, dass die Schliessung kaum über Monate hin aufrecht erhalten bleibt und dass das Restaurant deswegen in der Zukunft wieder Personal benötigen wird. Zudem war der Verwaltungsrat zufrieden mit den Leistungen der vier Arbeitnehmer und wollte sie weiter beschäftigen.

Kurzarbeit bedeutet eine Änderung des Arbeitsvertrags. Der Arbeitnehmer wird im Ergebnis freigestellt. ${ }^{49}$ Da der Arbeitgeber die Änderung nicht einseitig anordnen kann, ${ }^{50}$ fragte der Verwaltungsrat sämtliche Mitarbeiter an. Alle Mitarbeiter sagten zu und der Verwaltungsrat beantragte Kurzarbeitsentschädigung ab dem 16. März 2020. Für sich selbst beantragten die Verwaltungsratsmitglieder ebenfalls Kurzarbeitsentschädigung, allerdings erst ab 1. April 2020. (Die Verwaltungsratsmitglieder wollten die noch vorhandenen Vorräte verkaufen.) Dann reichte der Verwaltungsrat die Gesuche dem Zürcher Amt für Wirtschaft und Arbeit (AWA) ein. Dieses bewilligte die Kurzarbeitsentschädigung von $100 \%$ des Arbeitsausfalls für die vier Mitarbeiter für den halben Monat März 2020 und für alle sieben Mitarbeiter ab April 2020 bis Ende Juni.

\footnotetext{
46 Bundesgesetz über die obligatorische Arbeitslosenversicherung und die Insolvenzentschädigung (Arbeitslosenversicherungsgesetz, AVIG) vom 25. Juni 1982 (SR 837.0).

47 Siehe die Liste der öffentlich zugänglichen Einrichtungen in Art. 6 Abs. 2 und die Ausnahmen in Abs. 3 der COVID-19-Verordnung 2.

48 Art. 12 COVID-19-Verordnung 2.

49 GEISER/MÜLLER/PÄRLI, Rz. 512.

50 Statt aller ZK OR AV-STAEHELIN, Art. 322, N 44; siehe Art. 33 Abs. 1 lit. d AVIG.
} 
Die Kurzarbeitsentschädigung beträgt $80 \%$ des anrechenbaren Verdienstausfalls (Art. 34 Abs. 1 AVIG) ${ }^{51}$ Die Regel lautet, dass der Arbeitgeber die Kurzarbeitsentschädigung vorschiesst und den Arbeitnehmern am Zahltagstermin ausrichtet (Art. 37 lit. a AVIG). Danach macht er den Entschädigungsanspruch seiner Arbeitnehmer bei der Kasse geltend (Art. 38 Abs. 1 AVIG). Dieser Ablauf wurde während der Coronavirus-Pandemie insofern umgestellt, als Arbeitgeber die Auszahlung der Kurzarbeitsentschädigung verlangen konnten, ohne sie vorschiessen zu müssen (Art. 6 COVID-19-Verordnung Arbeitslosenversicherung). Die Sozialversicherungsbeiträge (Arbeitnehmer- und Arbeitgeberbeiträge) berechnet auf $100 \%$ des vertraglich vereinbarten Lohns muss der Arbeitgeber dennoch einzahlen (Art. 37 lit. c AVIG), die Arbeitgeberanteile werden ihm von der Arbeitslosenkasse aber wieder vergütet (Art. 39 Abs. 2 AVIG).

Für unser Beispiel wird der Einfachheit halber angenommen, dass die Personalkosten auf $80 \%$ sinken und - aufgrund des Art. 6 COVID-19-Verordnung Arbeitslosenversicherung - von der Arbeitslosenkasse übernommen werden. Folglich übernimmt die Arbeitslosenkasse für den März 2020 die halben Lohnkosten der Mitarbeiter im Umfang von Fr. 8'000. ${ }^{2}$

In der zweiten Märzhälfte betrieben die drei Verwaltungsratsmitglieder einen Take-away. Sie erzielten mit den (auf Fr. 10'000 wertberichtigten) Vorräten einen Umsatz von erfreulichen Fr. 20'000.

\section{Bilanz und Erfolgsrechnung am 3I. März 2020}

Mit verbuchter Kurzarbeitsentschädigung sowie dem erreichten Umsatz präsentierte sich die März-Erfolgsrechnung der Gesellschaft neu wie folgt:

51 Das Geltendmachen der Kurzarbeitsentschädigung ist formularlastig und teilweise anspruchsvoll: Zur Anmeldung z.B. Formular 716.300 (Art. 59 Abs. 2 AVIV); für die Abrechnung Formular 716.303. Die Formulare stehen auf der Webseite $<$ https://www.arbeit.swiss $>$ zur Verfügung. Siehe die Wegleitung und die Berechnungsbeispiele in: Staatssekretariat für Wirtschaft SECO (Kurzarbeitsentschädigung. Information für Arbeitgeber und Arbeitgeberinnen, Ausgabe 2016).

$5280 \%$ von Fr. $201000 / 2$. 
Abb. 7

\begin{tabular}{|l|r|l|r|}
\hline Aufwand & CHF & Ertrag & CHF \\
\hline Warenaufwand $^{53}$ & $65^{\prime} 000$ & Warenertrag $^{54}$ & $45^{\prime} 000$ \\
\hline Personalaufwand $^{55}$ & $33^{\prime} 000$ & Kurzarbeitsentschädigung $^{56}$ & $8^{\prime} 000$ \\
\hline Miete & $10^{\prime} 000$ & Verlust & $55^{\prime} 000$ \\
\hline Total & $108^{\prime} 000$ & & $108^{\prime} 000$ \\
\hline
\end{tabular}

Ausgehend von der Bilanz per 29. Februar 2020 (Abb. 3) sah die Bilanz per 31. März 2020 folgendermassen aus:

Abb. 8

\begin{tabular}{|l|r|l|r|}
\hline Aktiven & CHF & Passiven & CHF \\
\hline Umlaufvermögen & & Fremdkapital & \\
\hline Flüssige Mittel & & & $70^{\prime} 000$ \\
\hline Vorräte & $100^{\prime} 000$ & Darlehen Bank & \\
\hline Anlagevermögen & 0 & & $10^{\prime} 000$ \\
\hline Mobiliar & & Darlehen Vermieter & \\
\hline EDV & $10^{\prime} 000$ & Eigenkapital & $120^{\prime} 000$ \\
\hline & & Bktienkapital & $-50^{\prime} 000$ \\
\hline Total & $1500^{\prime} 000$ & & $150^{\prime} 000$ \\
\hline
\end{tabular}

Die Bilanz zeigt, dass die Gesellschaft durch die Kurzarbeitsentschädigung den hälftigen Kapitalverlust abwenden konnte. Der Bilanzverlust beträgt Fr. 50'000

57 Fr. 85'000 (Stand am 29. Februar 2020) + Fr. 45'000 (Warenertrag im März) + Fr. 8'000 (Kurzarbeitsentschädigung im März) - Fr. 5'000 (Wareneinkauf) - Fr. 33'000 (Personalaufwand).

58 Stundung der März-Miete.

Fr. 5'000 (Einkauf) + Fr. 50'000 (Abschreibung) + Fr. 10'000 (Abnahme Restvorräte).

Umsatz der ersten März-Hälfte (Fr. 25'000) und Umsatz der zweiten März-Hälfte (Fr. 20'000).

Fr. 15'000 (Lohn Aktionäre) + Fr. 10'000 (halber Märzlohn der vier Mitarbeiter) + Fr. 8'000 (Kurzarbeitsentschädigung).

80\% von Fr. 10 '000. 
und der Wert der Aktiven ist Fr. 100'000. Der Wert der Aktiven übersteigt damit den Wert der Hälfte des Aktienkapitals, so dass der hälftige Kapitalverlust per 31. März 2020 nicht mehr bestand.

Der Verwaltungsrat war sich indessen bewusst, dass Ende März 2020 weder die Krise ausgestanden noch die Gesellschaft saniert ist. Da der Bundesrat ankündigte, am 8. April 2020 über weitere Massnahmen zu entscheiden, wurde die nächste Verwaltungsratssitzung auf dieses Datum angesetzt.

\section{Ruhe vor dem Sturm im April 2020}

Am 8. April 2020 informierte der Bundesrat die Bevölkerung, dass die Schliessung der Geschäfte und Restaurants um eine Woche verlängert wird, vom 19. April bis zum 26. April 2020. Wie es danach weitergeht, war am 8. April 2020 noch nicht abschätzbar.

Der Verwaltungsrat kam wieder zusammen. Die Mitglieder gingen für die Finanzplanung davon aus (Art. 716a Abs. 1 Ziff. 3 OR), dass das Restaurant den ganzen April geschlossen bleibt.

Die April-Erfolgsrechnung sieht folgendermassen aus:

Abb. 9

\begin{tabular}{|l|r|l|r|}
\hline Aufwand & CHF & Ertrag & CHF \\
\hline Warenaufwand & 0 & Warenertrag & 0 \\
\hline Personalaufwand $^{59}$ & $28^{\prime} 000$ & Kurzarbeitsentschädigung & $28^{\prime} 000$ \\
\hline Miete & $10^{\prime} 000$ & Verlust & $10^{\prime} 000$ \\
\hline Total & $38^{\prime} 000$ & & $38^{\prime} 000$ \\
\hline
\end{tabular}

Die Bilanz per 30. April 2020 veränderte sich nur um den Verlust von Fr. 10’000.

$5980 \%$ von Fr. 35'000 (der Einfachheit halber erhalten die Aktionäre ebenfalls $80 \%$ von Fr. 5'000 - anstatt lediglich 80\% des maximal versicherten Lohns). 
Abb. 10

\begin{tabular}{|l|r|l|r|}
\hline Aktiven & CHF & Passiven & CHF \\
\hline Umlaufvermögen & & Fremdkapital & \\
\hline Flüssige Mittel & 100 '000 & Darlehen Bank & $70^{\prime} 000$ \\
\hline Vorräte & 0 & Darlehen Vermieter & 20 \\
\hline Anlagevermögen & & Eigenkapital & \\
\hline Mobiliar & 40 '000 & Aktienkapital & 120 '000 \\
\hline EDV & $10^{\prime} 000$ & Bilanzverlust & -60 '000 \\
\hline Total & 150 '000 & & $150^{\prime} 000$ \\
\hline
\end{tabular}

Der Bilanzverlust stieg von Fr. 50'000 per 31. März 2020 (Abb. 8) auf Fr. 60'000. Ein hälftiger Kapitalverlust lag knapp nicht vor und auch über Liquidität verfügte die Gesellschaft genügend.

\section{Fortgang der Krise im Mai 2020}

\section{Erneute Standortbestimmung und Einschätzung der Zukunft}

Am 29. April 2020 gab der Bundesrat bekannt, dass ab dem 11. Mai 2020 Restaurants unter Auflagen ${ }^{61}$ wieder öffnen dürfen.

Erneut kam der Verwaltungsrat zusammen und freute sich über die planbare Wiedereröffnung. Zeitgleich traten zwei neue Probleme auf:

(1) Der Kundenberater der Bank rief an. Er erkundigte sich nach der wirtschaftlichen Lage, bat um das Reporting und informierte die Gesellschaft, dass sie auch als Start-up einen COVID-19-Kredit beantragen könne. ${ }^{62}$

60 Gestundete Mietzinse für März und April 2020.

61 Die einzelnen Gästegruppen bestehen aus maximal 4 Personen oder Familien mit Kindern, die Konsumation erfolgt ausschliesslich sitzend, ein Schutzkonzept liegt vor und kann eingehalten werden (Art. 6 Abs. 3bis und Art. 6a Verordnung 2 über Massnahmen zur Bekämpfung des Coronavirus [COVID-19] [COVID-19-Verordnung 2] [Transitionsschritt 2: Schulen und Einkaufsläden sowie Sportbereich]; Änderung vom 29. April 2020; AS 2020 1499).

62 Art. 3 Abs. 1 lit. a Verordnung zur Gewährung von Krediten und Solidarbürgschaften in Folge des Coronavirus (Verordnung zur Gewährung von Krediten und Solidarbürgschaften infolge des Coronavirus [COVID-19-Solidarbürgschaftsverordnung, Covid-19-SBüV] vom 25. März 2020, SR 951.261). 
(2) Die Gesellschaft musste neue Waren einkaufen und ging - wie zu Beginn - von Kosten von Fr. 60'000 aus (vorne Abb. 1). Dieser Einkauf belastete die Liquidität. ${ }^{63}$

Von der Bank hatte sich die Gesellschaft nach Gründung Fr. 70'000 geliehen, in Form eines festen Vorschusses ${ }^{64}$ (Abb. 1). Zur Sicherung des Kredits bürgten die drei Aktionäre solidarisch mit der Gesellschaft. ${ }^{65}$

Die Gesellschaft verpflichtete sich der Bank gegenüber, während der Vertragslaufzeit stets ein wirtschaftliches Eigenkapital ${ }^{66}$ von Fr. 80'000 zu halten. Zudem enthielt der Vertrag Regeln zum sogenannten Reporting. Damit gemeint war die Pflicht der Gesellschaft, die Bank kontinuierlich über ihre wirtschaftliche Lage zu unterrichten. Vereinbart wurden ein quartalsweises sowie halbjährliches Reporting, erstmals Ende Juni 2020. Die Gesellschaft hatte zugesagt, der Bank regelmässig Finanzkennzahlen zu liefern. ${ }^{67}$

Der Verwaltungsrat ging davon aus, dass die Bank demnächst weitere Sicherheiten verlangt oder die Kündigung des Darlehensvertrags androht, in jedem Fall aber der Gesellschaft die Inanspruchnahme eines COVID-19-Kredits empfiehlt. Die Gesellschaft könnte einen zinsfreien Bankkredit von Fr. 50'000 beantragen und sich so Liquidität beschaffen. ${ }^{68}$

Für die Beurteilung des künftigen Liquiditätsbedarfs traf der Verwaltungsrat Annahmen: Er schätzte, dass bei Wiedereröffnung des Restaurants die Menschen im ersten Moment öfters auswärts essen, wobei aufgrund der Abstandsregeln aber weniger Gäste bedient werden können. Trotz dieser Unsicherheit

Während der Einkauf zugleich bilanzneutral ist: Ein Aktivum (flüssige Mittel) wird eingetauscht gegen ein anderes (Vorräte) vom selben Wert, so dass ein Aktiventausch vorliegt. Auch „Festkredit“, bei dem es sich rechtlich um ein Darlehen handelt (ABEGG ET AL., 131).

65 Solidarbürgschaft nach Art. 496 OR (ABEGG ET AL., 278 f.).

66 Als „wirtschaftliches Eigenkapital“ definieren die Kreditverträge meist den Summenwert von Aktienkapital, Reserven, Gewinnvortrag und nachrangigen Aktionärsdarlehen.

67 Das Reporting pro Monat oder pro Quartal enthält in der Regel den Warenlagerbestand, Übersichten über Debitoren, Kreditoren und den geplanten Einkauf (Betrag und Fälligkeit), sowie eine Meldung des Monatsumsatzes mit Soll/Ist-Vergleich. Das halbjährliche Reporting umfasst oft einen Halbjahresabschluss bestehend aus Soll/Ist-Vergleich von Umsatz und EBITA. Das Jahresreporting beinhaltet regelmässig den Jahresabschluss und sofern die Gesellschaft revidiert wird - den Revisionsbericht.

Art. 7 Abs. 2 COVID-19-Solidarbürgschaftsverordnung; siehe Erläuterungen zur COVID-19-Solidarbürgschaftsverordnung, 11 f.: Anstatt dem Umsatzerlös für ein Jahr geht Art. 7 Abs. 2 für neu gegründete Gesellschaften von 10\% des Dreifachen der Jahresnettolohnsumme aus (10\% von 12 x Fr. 35'000 x 3), wobei der Betrag das Total von Fr. 500'000 nicht übersteigen kann. 
entschied sich der Verwaltungsrat, für Fr. 60'000 Vorräte einzukaufen, also gleich viel wie für die Eröffnung, und alle sieben Arbeitnehmer zu beschäftigen. Der Verwaltungsrat ging davon aus, dass die Gesellschaft an den erfolgreichen Eröffnungsmonat Februar 2020 anknüpfen kann. Den Umsatz schätzte der Verwaltungsrat im (verkürzten) Mai 2020 auf Fr. 40'000 und plante gestützt auf diese Annahme die Mai-Erfolgsrechnung und die Bilanz per 31. Mai 2020. Diese Hochrechnungen bildeten dann die Grundlage für die weitere Planung:

Abb. 11

\begin{tabular}{|l|r|l|r|}
\hline Aufwand & CHF & Ertrag & CHF \\
\hline Warenaufwand & $15^{\prime} 000$ & Warenertrag & $40^{\prime} 000$ \\
\hline Personalaufwand & $35^{\prime} 000$ & & \\
\hline Miete & $10^{\prime} 000$ & Verlust & $20^{\prime} 000$ \\
\hline Total & $600^{\prime} 000$ & & 60 '000 \\
\hline
\end{tabular}

Abb. 12

\begin{tabular}{|l|r|l|r|}
\hline Aktiven & CHF & Passiven & CHF \\
\hline Umlaufvermögen & & Fremdkapital & \\
\hline Flüssige Mittel $^{69}$ & $45^{\prime} 000$ & Darlehen Bank & $70^{\prime} 000$ \\
\hline Vorräte $^{70}$ & $45^{\prime} 000$ & Darlehen Vermieter & $30^{71} 000$ \\
\hline Anlagevermögen & & Eigenkapital & \\
\hline Mobiliar & $40^{\prime} 000$ & Aktienkapital & $120^{\prime} 000$ \\
\hline EDV & $10^{\prime} 000$ & Bilanzverlust & $-80^{\prime} 000$ \\
\hline Total & $140^{\prime} 000$ & & $140^{\prime} 000$ \\
\hline
\end{tabular}

Aufgrund des Periodenverlusts erlitt die Gesellschaft wiederum einen hälftigen Kapitalverlust: Das Eigenkapital betrug Fr. 40'000 und lag damit unter dem Wert der Hälfte des Aktienkapitals.

69 Fr. 100'000 (flüssige Mittel am 30. April 2020) - Fr. 60'000 (Wareneinkauf) - Fr. 35'000 (Personalaufwand) + Fr. 40'000 (Warenertrag).

70 Von den eingekauften Vorräten wurden Waren für Fr. 15'000 verbraucht.

71 Gestundete Mietzinse für März, April und Mai 2020. 


\section{Pro und contra des COVID-I9-Kredits}

Bevor sich der Verwaltungsrat mit dem festen Vorschuss der Bank befasste, diskutierte er die Möglichkeit eines COVID-19-Kredits. Die Gesellschaft schätzte ihre Liquidität per Ende Mai auf Fr. 45'000. Im Juni allerdings würden wiederum Löhne fällig werden (Fr. 35’000) und Miete (Fr. 10'000); ferner sind neue Vorräte einzukaufen (Fr. 15'000) - und das Vermieterdarlehen (Fr. 30'000) wird zur Rückzahlung fällig. Im Juni entsteht somit ein Liquiditätsbedarf von Fr. 90'000. Aus den vorhandenen Mitteln kann dieser Bedarf nicht gedeckt werden; somit muss der Umsatz die benötigte Liquidität in die Kasse bringen.

Der Verwaltungsrat diskutierte, ob im Juni weniger Leute auswärts essen und die Gefahr, dass die Restaurants aufgrund einer neuen Krankheitswelle erneut schliessen müssen. Die Gesellschaft sass auf einem dünnen Liquiditätspolster und dieses sprach für die Inanspruchnahme eines COVID-19-Kredits. Der geliehene Betrag würde zudem für die Berechnung der Schwellenwerte in Art. 725 OR nicht als Fremdkapital berücksichtigt (Art. 24 COVID-19-Solidarbürgschaftsverordnung). Aber die Verschuldung der Gesellschaft nähme zu. Auch dürfte der COVID-19-Kredit nicht zur ausserordentlichen Rückzahlung des bestehenden Bankkredits (Refinanzierung) verwendet werden (Art. 6 Abs. 3 lit. b COVID-19-Solidarbürgschaftsverordnung). Und letztlich wäre der Kredit bilanzneutral; d.h., dass durch die Auszahlung die Aktiven (flüssige Mittel) um Fr. 50'000 zunehmen, die Passiven (Bankschulden) aber um denselben Betrag ebenfalls. Somit würde der Kredit nicht helfen, das im Bankvertrag vereinbarte wirtschaftliche Eigenkapital zu erreichen: Der Bilanzverlust bliebe gleich aufgrund der Erhöhung der Aktiven und der Passiven.

Der Verwaltungsrat beschloss, auf einen COVID-19-Kredit zu verzichten und andere Finanzierungslösungen zu suchen.

\section{Innenfinanzierung}

\section{a) Kapitalschnitt}

Die Verwaltungsratsmitglieder und Aktionäre fragten sich, ob die Sanierung mit eigenem Geld möglich ist. Der erste Aktionär könnte der Gesellschaft Liquidität von Fr. 30'000 zur Verfügung stellen, der zweite Aktionär Fr. 15’000. Der dritte Aktionär besass zu wenig eigenes Vermögen und konnte sich nicht beteiligen.

Damit entfiel bereits eine Sanierungsmassnahme: der Kapitalschnitt. Beim Kapitalschnitt wird das Aktienkapital auf null herabgesetzt und anschliessend 
neu einbezahlt (Art. 732a OR). ${ }^{72}$ Das Aktienkapital betrug Fr. 120'000, was verteilt auf drei Aktionäre je Fr. 40'000 ausmacht. Aufgrund der finanziellen Verhältnisse der drei Aktionäre wurde der Kapitalschnitt nicht weiter besprochen.

\section{b) Ablösung der kreditgebenden Bank}

Da die Aktionäre gemeinsam Fr. 45’000 für die Sanierung aufbringen konnten, entfiel auch die Möglichkeit, den Bankkredit zu kündigen und der Gesellschaft einen Betriebskredit selbst zur Verfügung zu stellen. Rechnerisch wäre zwar denkbar gewesen, die Hälfte des Kredits zurückzuzahlen und durch Aktionärsdarlehen zu ersetzen, doch auch dadurch hätte sich die Liquidität nicht verbessert.

Die Ablösung eines alten Kreditgebers kommt grundsätzlich nur infrage, wenn die Überschuldung droht und der neue Gläubiger, z.B. ein Aktionär, zu einem Rangrücktritt bereit ist und der alte Gläubiger dies ablehnt. Da im vorliegenden Beispiel die Überschuldung noch nicht unmittelbar bevorsteht, bringt die Ablösung des halben Bankkredits kaum einen Vorteil. ${ }^{73}$

\section{c) Kapitalzuschuss oder Sanierungsdarlehen}

Die Aktionäre diskutierten folglich Zuschüsse und Sanierungsdarlehen.

Unter einem Kapitalzuschuss versteht man einen Betrag, der meist von einem Gläubiger à fonds perdu an die Gesellschaft gezahlt wird. ${ }^{74}$ Ein Sanierungsdarlehen wird einem Schuldner zum besonderen Zweck der Sanierung gewährt und kann privilegiert zurückbezahlt werden. ${ }^{75}$

Die Verwaltungsratsmitglieder verwarfen die Idee der Kapitalzuschüsse, da diese zu unterschiedlichen Sanierungsbeiträgen ohne Gegenleistung führen und dies als ungerecht empfunden wurde. Der Verwaltungsrat erstellte daher eine Bilanz unter Berücksichtigung von Sanierungsdarlehen über Fr. 20'000 und Fr. 15’000. Die Bilanz würde sich per 31. Mai 2020 wie folgt präsentieren:

72 BGE 138 III 204.

73 Allenfalls denkbar ist eine Zinsersparnis, wenn die kreditgebenden Aktionäre der Gesellschaft das Kapital zinsfrei zur Verfügung stellen.

74 KÄLIN, Rz. 220 ff., mit Hinweisen; zur Verbuchung HANDSCHIN, Rz. 850; zur steuerrechtlichen Erfassung BSK DBG-HELBING/FELBER, Art. 67, N 52 ff., N 54.

75 BGE 134 III 452 E. 5.2 f.; BGE 99 III 27 E. 5; MÜLLER, Rz. 114 ff.; EMMENEGGER, 157 ff. 
Abb. 13

\begin{tabular}{|l|r|l|r|}
\hline Aktiven & CHF & Passiven & CHF \\
\hline Umlaufvermögen & & Fremdkapital & \\
\hline Flüssige Mittel & $90^{\prime} 000$ & Darlehen Bank & $70^{\prime} 000$ \\
\hline Vorräte & $45^{\prime} 000$ & Darlehen Vermieter & $30^{\prime} 000$ \\
\hline & & Darlehen Aktionäre & $45^{\prime} 000$ \\
\hline Anlagevermögen & & Eigenkapital & \\
\hline Mobiliar & $40^{\prime} 000$ & Aktienkapital & 120 '000 \\
\hline EDV & $10^{\prime} 000$ & Bilanzverlust & $-80^{\prime} 000$ \\
\hline Total & $185^{\prime} 000$ & & $185^{\prime} 000$ \\
\hline
\end{tabular}

Der Bilanzverlust von Fr. 80'000 würde trotz Sanierungsdarlehen bestehen bleiben. Die Gesellschaft benötigte folglich Eigenkapital. Da die beiden Aktionäre, die Geld zur Verfügung stellen könnten, zu Zuschüssen nicht bereit waren, besprachen die Verwaltungsratsmitglieder die Möglichkeit eines Debt Equity Swaps.

\section{d) Debt Equity Swap}

Der Debt Equity Swap bezeichnet die Umwandlung von Fremd- in Eigenkapital: Die Gesellschaft erhöht ihr Gesellschaftskapital und der Geldgeber erhält Gesellschaftsanteile als Gegenleistung für das Geld, das er in die Gesellschaft einzahlt. $^{76}$

Die Verwaltungsratsmitglieder/Aktionäre in unserem Beispiel einigten sich auf einen Debt Equity Swap. Diejenigen Aktionäre, die der Gesellschaft Geld zur Verfügung stellten, sollten mehr Aktien erhalten. Sie partizipierten damit unmittelbar am künftigen Erfolg oder Misserfolg der Gesellschaft.

Die Gesellschaft führte eine Kapitalerhöhung durch (Art. 650 OR) und erhöhte das Aktienkapital von Fr. 120'000 auf Fr. 165'000. Die Bezugsrechte wurden ausgeschlossen (Art. 704 Abs. 1 Ziff. 6 OR) und die Liberierung der Aktien geschah durch Verrechnung. ${ }^{77}$

76 Im Einzelnen KÄLIN, Rz. 187 ff.

77 Zur Werthaltigkeit der Verrechnungsforderung siehe Botschaft des Bundesrates vom 23. November 2016 zur Änderung des Obligationenrechts (Aktienrecht), BBl 2017, 399 ff., 463. 
Nach dem Debt Equity Swap sah die Bilanz per 31. Mai 2020 folgendermassen aus:

Abb. 14

\begin{tabular}{|l|r|l|r|}
\hline Aktiven & CHF & Passiven & CHF \\
\hline Umlaufvermögen & & Fremdkapital & \\
\hline Flüssige Mittel & $90^{\prime} 000$ & Darlehen Bank & $70^{\prime} 000$ \\
\hline Vorräte & $45^{\prime} 000$ & Darlehen Vermieter & $30^{\prime} 000$ \\
\hline Anlagevermögen & & Eigenkapital & \\
\hline Mobiliar & $40^{\prime} 000$ & Aktienkapital & $165^{\prime} 000$ \\
\hline EDV & $10^{\prime} 000$ & Bilanzverlust & $-80^{\prime} 000$ \\
\hline Total & $185^{\prime} 000$ & & $185^{\prime} 000$ \\
\hline
\end{tabular}

Das Aktienkapital betrug neu Fr. 165'000. Das Eigenkapital stieg um Fr. 45’000 auf Fr. 85'000. Ein hälftiger Kapitalverlust bestand nicht mehr und die Gesellschaft erfüllte die Bedingung der Bank bezüglich Mindesthöhe des wirtschaftlichen Eigenkapitals.

\section{Einstweiliger Abschluss im Juni 2020}

\section{Erfolgsrechnung, Bilanz und die Folgen}

Auch im Juni konnte die Gesellschaft noch nicht an den Erfolg im Februar 2020 anknüpfen. Das Restaurant erzielte einen Umsatz von Fr. 55'000. Vorräte wurden im Wert von Fr. 15'000 verbraucht und im gleichen Umfang wieder eingekauft.

Der Vermieter offerierte, den Betrag der gestundeten Mietzinse für die Monate März, April und Mai (Fr. 30'000) um insgesamt Fr. 5'000 zu reduzieren (unter der Bedingung, dass die Gesellschaft die Schuld umgehend begleicht). Der Verwaltungsrat nahm das Angebot an. Die Erfolgsrechnung für den Juni sah so aus: 
Abb. 15

\begin{tabular}{|l|r|l|r|}
\hline Aufwand & CHF & Ertrag & CHF \\
\hline Warenaufwand & $15^{\prime} 000$ & Warenertrag & $55^{\prime} 000$ \\
\hline Personalaufwand & $35^{\prime} 000$ & Ausserordentlicher Ertrag & $5{ }^{\prime} 000$ \\
\hline Miete & $10^{\prime} 000$ & Gewinn/Verlust & 0 \\
\hline Total & $60^{\prime} 000$ & & $60^{\prime} 000$ \\
\hline
\end{tabular}

Als Folge präsentiert sich die Bilanz per 30. Juni 2020 folgendermassen:

Abb. 16

\begin{tabular}{|l|r|l|r|}
\hline Aktiven & CHF & Passiven & CHF \\
\hline Umlaufvermögen & & Fremdkapital & \\
\hline Flüssige Mittel $^{78}$ & $60^{\prime} 000$ & Darlehen Bank & $70^{\prime} 000$ \\
\hline Vorräte & $45^{\prime} 000$ & Darlehen Vermieter & 0 \\
\hline Anlagevermögen & & Eigenkapital & \\
\hline Mobiliar & $40^{\prime} 000$ & Aktienkapital & $165^{\prime} 000$ \\
\hline EDV & $10^{\prime} 000$ & Bilanzverlust & $-80^{\prime} 000$ \\
\hline Total & $155^{\prime} 000$ & & $155^{\prime} 000$ \\
\hline
\end{tabular}

Durch den Umsatz im Juni 2020 schrumpfte die Bilanz auf Fr. 155’000. Der Bilanzverlust blieb gleich und das Eigenkapital betrug weiterhin Fr. 85’000. Eine Überschuldung drohte nicht mehr und auch ein hälftiger Kapitalverlust lag ausser Reichweite.

Der Verwaltungsrat war sich indessen bewusst, dass lediglich durch den Forderungsverzicht des Vermieters ein neutrales Ergebnis erzielt wurde. Sollte es nicht gelingen, in der nächsten Zeit Gewinne zu erwirtschaften, würde die Gesellschaft umgehend wieder Richtung Kapitalverlust und Überschuldung rutschen. Schweren Herzens beschloss der Verwaltungsrat daher, zwei Mitarbeiter zu entlassen und beim Vermieter auf dem Verhandlungsweg zu versuchen, den Mietzins dauerhaft zu senken.

78 Fr. 90'000 (Stand am 31. Mai 2020) - Fr. 25'000 (um Fr. 5'000 reduzierte Mietzinsschuld März, April und Mai) - Fr. 15’000 (Wareneinkauf) - Fr. 35'000 (Personalaufwand) - Fr. 10'000 (Miete Juni) + Fr. 55'000 (Warenertrag). 


\section{Aussicht und Ergebnis}

Das Restaurant hielt der Coronakrise stand, eine sanierte Gesellschaft lag aber noch nicht vor. Zum einen blieb unklar, ob die Behörden erneut Schliessungen anordnen, zum anderen war der Umsatz in den kommenden Wochen und Monaten schwer zu prognostizieren. Zum Zeitpunkt des Fertigstellens dieses Aufsatzes am 30. September 2020 ist die Zukunft unsicher.

Die Gesellschaft war zudem nicht mehr die gleiche: Das Aktionariat veränderte sich und die drei Gründer waren neu unterschiedlich beteiligt. Der erste Aktionär besass 40 Aktien, der zweite 55 und der dritte 60. Aufgrund dieser weiterhin recht gleichmässigen Aktienstreuung blieben die Kräfteverhältnisse weiterhin ähnlich, haben sich aber verschoben.

Erkenntnisse sind die folgenden: (1) Liquidität und Vermögen sind zweierlei. Auch im diskutierten Beispiel zeigt sich, wie stark die Liquidität in einer Sanierungssituation zu beachten ist. (2) Die Sanierung verändert eine Gesellschaft praktisch immer, entweder auf der Eigenkapital- oder der Fremdkapitalseite. (3) Verluste sind und bleiben das wichtigste Krisenmerkmal. (4) Der COVID-19-Kredit kann sich als Bumerang erweisen, wenn zwar der Betrieb aufrechterhalten wird, die Fixkosten das Geld aber verbrennen. Diesfalls resultiert kein Mehrwert für die Schuldnerin und die Verschuldung steigt an.

\section{Literaturverzeichnis}

ABEGG PHILIPP ET AL., Schweizerisches Bankenrecht, 4. A., Zürich/Basel/Genf 2019.

Basler Kommentar, Bundesgesetz über die direkte Bundessteuer (DBG), in: Zweifel Martin/ Beusch Michael (Hrsg), 3. A., Basel 2017 (zit. BSK DBG-BEARBEITER, Art. XX, N YY).

Basler Kommentar, Obligationenrecht II, in: Honsell Heinrich/Vogt Nedim Peter/Watter Rolf (Hrsg.), 5. A., Basel 2016 (zit. BSK OR II-BEARBEITER/in, Art. XX, N YY).

BoEmle MaX/LutZ Ralf, Der Jahresabschluss, 5. A., Zürich 2008.

BÖCKLI PEter, Schweizer Aktienrecht, 4. A., Zürich/Basel/Genf 2009 (zit. BÖcKLI, Aktienrecht).

BÖCKLI PETER, OR-Rechnungslegung, 2. A., Zürich/Basel/Genf 2019 (zit. BÖcKLI, Rechnungslegung).

EMmEnegger SusAn, Das Sanierungsdarlehen, in: Emmenegger Susan (Hrsg.), Kreditrecht, Schweizerische Bankrechtstagung 2010, Basel 2010, 153-189.

Geiser Thomas/Müller Roland/Pärli Kurt, Arbeitsrecht in der Schweiz, 4. A., Bern 2019.

HANDSCHIN LUKAS, Rechnungslegung im Gesellschaftsrecht, 2. A., Basel 2016.

KÄLIN OLIVER, Die Sanierung der Aktiengesellschaft, Zürich/Basel/Genf 2016.

MEYER CONRAD, Finanzielles Rechnungswesen, 4. A., Zürich 2020. 
MÜLLER KARIN, Eigenkapitalersetzende Darlehen, Dogmatische Grundlagen und praktische Konsequenzen, Habil., Bern 2014.

Rechnungslegung nach Obligationenrecht, veb.ch Praxiskommentar, in: Pfaff Dieter et al. (Hrsg.), 2. A., Zürich 2019 (zit. BEARBEITER, veb.ch Praxiskommentar, Art. XX, N YY).

SCHELLENBERG ALDO C., Rechnungswesen, 4. A., Zürich 2010.

Schweizer Handbuch der Wirtschaftsprüfung, Band „Buchführung und Rechnungslegung“, Zürich 2014 (zit. HWP).

THOMmen JeAn-PaUL, Betriebswirtschaft und Management, 10. Aufl., Zürich 2016.

Zürcher Kommentar, Obligationenrecht, Die Aktiengesellschaft, Der Verwaltungsrat, Art. 707-726 OR, in: Tanner Brigitte et al. (Hrsg.), Zürich 1997 (zit. ZK OR AG-BEARBEITER, Art. XX, N YY).

Zürcher Kommentar, Obligationenrecht, Die Miete, Vorbemerkungen zum 8. Titel (Art. 253-273c OR), Art. 252-265 OR, in: Higi Peter/Bühlmann Anton/Wildisen Christoph (Hrsg.), 5. A., Zürich/Basel/Genf 2019 (zit. ZK OR-BEARBEITER, Art. XX, N YY). 



\title{
Das Stillhalteabkommen - insolvenzrechtliche Aspekte
}

\author{
Marc Bernheim/Gaudenz Geiger
}

\section{Inhalt}

I. Einleitung 93

II. Das Stillhalteabkommen $\quad 94$

1. Das Stillhalteabkommen als Sanierungsinstrument 94

2. Inhalt eines Stillhalteabkommens $\quad 96$

III. Insolvenzrechtliche Problemfelder 97

1. Paulianische Anfechtung 98

a) Überschuldungsanfechtung (Art. 287 SchKG)

a) Informationsrechte 100

bb) Handlungen i.S.v. Art. 287 SchKG 101

b) Absichtsanfechtung (Art. 288 SchKG) 104

aa) Gläubigerschädigung 105

bb) Schädigungsabsicht und Erkennbarkeit der Schädigungsabsicht $\quad 107$

cc) Sanierungsdarlehen 109

2. Aktienrechtliche Verantwortlichkeit 111

a) Faktische Organschaft 112

a) Allgemeines 112

bb) Relevanz bei Stillhalteabkommen 113

b) Tatbestandsmerkmale und Haftung $\quad 115$

aa) Allgemeines 115

bb) Relevanz für Stillhalteabkommen $\quad 116$

IV. Fazit 118

\begin{tabular}{ll} 
Literaturverzeichnis & 119 \\
\hline
\end{tabular}

\section{Einleitung}

Befindet sich ein Unternehmen in finanzieller Schieflage, so sind Sanierungsmassnahmen zu ergreifen. Ziel der Sanierung ist die Überwindung der finanziellen Krise des Unternehmens, wobei eine Sanierung in der Regel (nur) dann an die Hand genommen wird, wenn das Unternehmen sanierungswürdig ist. Mit anderen Worten dann, wenn der Wert des sanierten Unternehmens abzüglich der Kosten der Sanierung höher liegt, als der ohne Sanierung erzielbare Liquidationserlös. 
Private Sanierungsmassnahmen, also Massnahmen ausserhalb eines Konkursoder Nachlassverfahrens, stellen dabei die schonendste Art der Sanierung dar, da solche Massnahmen die operative Unternehmenstätigkeit weder unterbrechen noch negativ beeinflussen und damit der Unternehmenswert bestmöglich erhalten bleibt.

Der vorliegende Beitrag befasst sich mit den insolvenzrechtlichen Aspekten von Stillhalteabkommen als private Sanierungsmassnahme. Namentlich mit dem Fall, dass die Sanierung einer Unternehmung trotz Abschluss eines Stillhalteabkommens scheitert und die Insolvenz über das Unternehmen eröffnet wird. In dieser Konstellation stellen sich für die am Stillhalteabkommen teilnehmenden Gläubiger verschiedene Fragen. Insbesondere stellt sich die Frage, ob unter dem Stillhalteabkommen getätigte Rechtshandlungen der insolvenzrechtlichen Anfechtung unterliegen, und ob sich die am Stillhalteabkommen teilnehmenden Gläubiger dem Risiko einer aktienrechtlichen Verantwortlichkeit aussetzen.

\section{Das Stillhalteabkommen}

\section{Das Stillhalteabkommen als Sanierungsinstrument}

Ein Stillhalteabkommen stellt ein privatrechtliches Sanierungsinstrument dar, welches insbesondere im Verhältnis zwischen dem Schuldner und seinen Finanzgläubigern relevant ist, regelmässig eine oder mehrere Banken. ${ }^{1}$ Mit dem Stillhalteabkommen versuchen die Banken ihre Kreditpositionen zu verbessern; gleichzeitig stellen Stillhalteabkommen der am häufigsten angewendete Sanierungsbeitrag dar. ${ }^{2}$

Der Kern eines Stillhalteabkommens besteht in der Abrede zwischen den Finanzgläubigern bzw. Kreditgebern und dem Kreditnehmer, die gewährten

1 Wobei sich vielfach nur ungesicherte (oder ungenügend gesicherte) Gläubiger an einer Sanierung beteiligen werden. Gesicherte Gläubiger haben in einem allfälligen Konkursoder Nachlassverfahren nur geringe oder keine Verluste zu befürchten, weshalb sie kaum bereit sind, im Rahmen eines Sanierungsverfahrens Leistungen zu erbringen. Andere Gläubiger - etwa Lieferanten und Dienstleister - werden regelmässig nicht in Sanierungen (auch nicht in Stillhalteabkommen) involviert, obwohl auch ihre Forderungen ungesichert und in einem Insolvenzverfahren ungeschützt sind. Einerseits sind die Forderungen solcher Gläubiger regelmässig klein, was einen grossen Aufwand bei kleinem Nutzen bei der Sanierung zur Folge hat. Andererseits ist die vollständige Befriedigung von Lieferanten, Dienstleistern etc. regelmässig Voraussetzung dafür, dass eine fortgesetzte Belieferung während und nach erfolgter Sanierung erfolgt, was für das Überleben des Schuldners zentral ist.

2 Urteil des Bundesgerichts 5A_671/2018 vom 8. September 2020 E. 3.1.1. 
Kreditlimiten für eine bestimmte Zeitdauer und zu den vereinbarten Bedingungen offen zu halten. ${ }^{3}$ Da zum Zeitpunkt des Abschlusses eines Stillhalteabkommens regelmässig bereits Verstösse gegen den Kreditvertrag bzw. die Kreditbedingungen vorliegen, verzichten die Finanzgläubiger im Stillhalteabkommen vorübergehend auf die Ausübung ihres Kündigungsrechts. Das Stillhalteabkommen beinhaltet mit anderen Worten im Wesentlichen zwei Elemente: Einerseits verzichtet der Finanzgläubiger auf die Kündigung seiner Kreditforderung, andererseits stundet er bereits fällig gewordene oder in Zukunft fällig werdende Forderungen ${ }^{4}$ für die Dauer des Stillhalteabkommens. ${ }^{5}$ Sperren die Finanzgläubiger ihre Kreditlinien, so fehlt es an der für die Fortführung des Geschäfts notwendigen Liquidität. Dies führt im Ergebnis zur Umstellung der Bilanzierung von Fortführungs- zu Liquidationswerten, was regelmässig zu einer Überschuldungssituation führt. ${ }^{6}$ Das Offenhalten von bestehenden Kreditlinien ist deshalb für das Gelingen einer Sanierung zentral. Entsprechend stark ist denn auch regelmässig die Position der Finanzgläubiger bei der Aushandlung eines Stillhalteabkommens.

Dem Kreditnehmer wird durch das Stillhalteabkommen ein zeitlicher Handlungsspielraum eingeräumt, um weitere Massnahmen zur Sanierung des Unternehmens voranzutreiben. ${ }^{7}$ Genügt die Stundung der Finanzgläubiger nicht, um die Existenz des Schuldners zu erhalten, sondern benötigt dieser zusätzliche Liquidität (die den bereits bestehenden Kreditrahmen übersteigt), so kann das Stillhalteabkommen auch mit einem Überbrückungskredit kombiniert werden. ${ }^{8}$

Ein Stillhalteabkommen, bei dem die Finanzgläubiger bis zu einem bestimmten Zeitpunkt auf die Zahlung fälliger Forderungen verzichten, kann normalerweise nur abgeschlossen werden, wenn sämtliche Finanzgläubiger einbezogen werden. Werden nicht alle Finanzgläubiger mit einbezogen, und werden daher

3 Ein Stillhalteabkommen hat also keinen Einfluss auf die Bilanzierung der betroffenen Forderung und hat deshalb auch keine Auswirkung auf das Bilanzbild und die Pflichten gemäss Art. 725 OR. Vgl. SPRECHER/SOMMER, $58 \mathrm{f}$.

4 Z.B. vertraglich vereinbarte Zins-, Gebühren- und Amortisationszahlungen. Möglich ist, gewisse Forderungen von der Stundungsabrede auszunehmen (z.B. Forderungen, die im Zusammenhang mit einem Überbrückungskredit stehen).

5 RUBLI, 179.

6 Vgl. FisCHER R., 44

7 SCHMID, 176. Ein Stillhalteabkommen dient regelmässig (nur) zur Überbrückung der Zeitspanne, bis eine umfassende Sanierung und Umschuldung vorgenommen werden kann, stellt mit anderen Worten also regelmässig nur den ersten Schritt eines Sanierungsvorgangs dar.

8 SCHENKER, Restrukturierung, 67. 
einzelne Finanzgläubiger von der Gesellschaft noch befriedigt, so werden in der Folge auch alle anderen Finanzgläubiger eine entsprechende Zahlung verlangen und damit eine Sanierung ausserhalb des Insolvenzrechts verunmöglichen. $^{9}$

\section{Inhalt eines Stillhalteabkommens}

Eine Stillhaltevereinbarung ist eine vertragliche Änderung bzw. Ergänzung eines Grundvertrages, meistens eines Kreditvertrages. Im Stillhalteabkommen verpflichten sich die Finanzgläubiger, während der Dauer des Abkommens ihre bereits gewährten Kreditlinien offenzuhalten und nicht zu kürzen. Die Finanzgläubiger verzichten damit nicht nur darauf, fällige Forderungen einzutreiben, sondern ermöglichen es der Gesellschaft, noch unbenützte Kreditlinien zu nutzen..$^{10}$ Regelmässig wird sodann vereinbart, dass die teilnehmenden Finanzgläubiger allfällige Verluste (die in einem Konkurs- oder Nachlassverfahren während der Dauer des Abkommens entstehen) anteilig tragen werden, dass die Kreditbedingungen (insb. die Zinsen) der verschiedenen Finanzgläubiger vereinheitlicht werden. Zudem wird häufig ein Upside-Potential für die teilnehmenden Finanzgläubiger für den Fall des Gelingens der Sanierung vereinbart.

Ebenfalls enthalten Stillhalteabkommen regelmässig diverse Verpflichtungen der Kreditnehmerin (sog. Covenants), wobei der Inhalt dieser Verpflichtungen vom jeweiligen Einzelfall abhängig ist. ${ }^{11}$ Üblich ist etwa die Verpflichtung zur Einleitung gewisser Sanierungs- und Restrukturierungsmassnahmen ${ }^{12}$, Vorga-

9 SCHENKER, Restrukturierung, 66. Auch kann der Abschluss eines Stillhalteabkommens (und teilweise sogar bereits die Einleitung von Verhandlungen um ein Stillhalteabkommen mit Gläubigerbanken) einen Verzugsfall („event of default“) auslösen, der unter Umständen zu gravierenden vertraglichen Rechtsfolgen führt. Auch zur Vermeidung solcher Spillover bzw. Cross Default Effekte ist es regelmässig notwendig, sämtliche Finanzgläubiger in einem Stillhalteabkommen zu berücksichtigen. Zu dieser Problematik: RUBLI, 179.

Allgemein zum Inhalt von Stillhalteabkommen: SCHENKER, Restrukturierung, 67 ff., AFFENTRANGER, 118 ff., RuBli, 178 ff., WOlF, N 252 ff., FisCHER R., 45 ff., Urteil des Bundesgerichts 5A_671/2018 vom 8. September 2020 E. 3.1.1, Urteil des Bundesgerichts 4C.47/2003 vom 2. Juli 2003.

11 Ausführlich: WOLF, N 274 ff.; SCHENKER, privatrechtlichen Sanierung, $491 \mathrm{f}$.

12 Z.B. die Umsetzung eines vorab kommunizierten Turnaround-Konzepts oder die Erreichung gewisser, vordefinierter Zielgrössen. 
ben zur Verwendung der vorhandenen finanziellen Mittel ${ }^{13}$, die Verpflichtung zu Devestitionen und Kreditrückführungen ${ }^{14}$, die Pflicht zur zusätzlichen Besicherung von Krediten ${ }^{15}$, ein Genehmigungsvorbehalt der Finanzgläubiger für gewisse Geschäfte ${ }^{16}$, die Verpflichtung zur Information der Finanzgläubiger, die Pflicht einen Sanierungsberater beizuziehen oder die Pflicht zur Schaffung eines Steuerungsausschusses.

\section{Insolvenzrechtliche Problemfelder}

Stillhalteabkommen können - je nach konkretem Inhalt und Zeitpunkt des Abschlusses - insbesondere unter dem Gesichtspunkt der paulianischen Anfechtung (Art. 285 ff. SchKG) und der aktienrechtlichen Verantwortlichkeit (Art. 752 ff. OR) relevant sein. Dies in aller Regel aber nur dann, wenn die beabsichtigte (und mittels dem Stillhalteabkommen zumindest unterstützte) Sanierung des Unternehmens scheitert. Fragen um die paulianische Anfechtung und die aktienrechtliche Verantwortlichkeit im Zusammenhang mit Stillhalteabkommen stellen sich mit anderen Worten dann, wenn das zu sanierende Unternehmen in Konkurs fällt, oder wenn es zu einem Nachlassvertrag mit Vermögensabtretung kommt. In aller Regel kein Thema sind paulianische Anfechtungsklagen und die Verantwortlichkeit demgegenüber wenn ein ordentlicher Nachlassvertrag (Dividenden- oder Stundungsvergleich i.S.v. Art. 314 ff. SchKG) abgeschlossen wird. ${ }^{17}$ Dies macht den Abschluss eines ordentlichen Nachlassvertrags für den Schuldner bzw. die verantwortlichen Organe des Schuldners zu einem besonders erstrebenswerten Ergebnis eines Insolvenzverfahrens.

13 Es kann vereinbart werden, für was die vorhandenen Mittel verwendet werden dürfen (z.B. zur Befriedigung von Lieferanten und Dienstleistern) oder für was nicht (z.B. zur Ausschüttung an die Aktionäre oder zur Befriedigung von Finanzgläubigern, die sich am Stillhalteabkommen nicht beteiligen).

14 Z.B. die Verpflichtung, den Verwertungserlös gewisser Aktiven zur Rückführung der vom Stillhalteabkommen erfassten Kredite zu verwenden.

15 Relevant ist insbesondere die Pflicht, für die vom Stillhalteabkommen erfassten Kredite zusätzliche Sicherheiten zu stellen.

16 D.h. die Pflicht, für die Durchführung gewisser Geschäfte die vorgängige Genehmigung der am Stillhalteabkommen teilnehmenden Kreditgeber einzuholen (etwa, dass ohne die Zustimmung der Finanzgläubiger keine grösseren Investitionen und Akquisitionen getätigt und keine neuen Sicherheiten eingeräumt werden).

17 Dazu, auch zu den Ausnahmefällen: SK SchKG-HunKELER/WoHL, Art. 314 SchKG, N 6 f. 


\section{Paulianische Anfechtung}

Mittels der paulianischen Anfechtungsklagen sollen Vermögenswerte der Zwangsvollstreckung zugeführt werden, die ihr durch eine Rechtshandlung gemäss Art. 286 bis 288 SchKG entzogen worden sind (Art. 285 Abs. 1 SchKG). Bezweckt wird die Wiederherstellung des Vermögens des Schuldners, wie es ohne die anfechtbare Handlung vorhanden gewesen wäre. Vermögenswerte, die der Schuldner zuvor durch zivilrechtlich gültige, aber paulianisch anfechtbare Rechtsakte veräusserte, sollen der Zwangsvollstreckung zugeführt werden. Die anfechtbaren Handlungen behalten dabei zwar ihre zivilrechtliche Gültigkeit, werden aber betreibungsrechtlich unbeachtlich. ${ }^{18}$

Objektive Voraussetzung jeder Anfechtungsklage ist, dass die anfechtbare Rechtshandlung einen oder mehrere Gläubiger schädigt, indem das Vollstreckungssubstrat vermindert, oder die Stellung eines oder mehrerer Gläubiger im Vollstreckungsverfahren in einer anderen Weise verschlechtert wird. Die Schädigung kann in einer Verminderung der verwertbaren Aktiven oder in einer Vermehrung der Passiven bestehen. ${ }^{19}$ Die tatsächliche Schädigung wird nach der Praxis des Bundesgerichts zu Gunsten der Konkursmasse vermutet und muss vom Kläger nicht nachgewiesen werden. Diese Vermutung kann der Anfechtungsbeklagte durch den Gegenbeweis, dass die anderen Gläubiger auch bei richtigem Verhalten des Schuldners geschädigt worden wären, widerlegen.$^{20}$ Keine Gläubigerschädigung liegt in aller Regel dann vor, wenn gleichwertige Leistungen ausgetauscht werden. ${ }^{21}$

Die Gefahr eines Konkurses oder eines Nachlassverfahrens führt dazu, dass die am Stillhalteabkommen Beteiligten die Restriktionen beachten müssen, die sich aus den Anfechtungsklagen (Art. 285 ff. SchKG) ergeben. ${ }^{22}$ Fällt der Kreditnehmer trotz der Sanierungsbemühungen in Konkurs oder wird ein Nachlassvertrag mit Vermögensabtretung abgeschlossen, so können die am Stillhalteabkommen teilnehmenden Finanzgläubiger unter gewissen Umständen mittels Anfechtungsklagen verpflichtet werden, die vor der Insolvenz des Kreditschuldners erhaltenen Leistungen zurückzuerstatten. Als anfechtbare

Statt aller: KUKO SchKG-UmBaCh-SPAHn/BossaRT, Art. 285 SchKG, N 1.

KUKO SchKG-UmBaCh-SPAHN/BOSSART, Art. 285 SchKG, N 4. Vgl. auch KRIESI, 49, die zutreffend konkretisiert, dass eine Verschlechterung voraussetzt, dass die Gläubiger in der Zwangsvollstreckung einen Ausfall erlitten haben, der nicht oder in geringerer Höhe vorläge, wenn man die konkrete Handlung wegdenken würde.

GLANZMANN, 264.

Es sei denn, der Schuldner habe mit dem Geschäft bezweckt, zum Schaden der Gläubiger über seine letzten Vermögenswerte zu verfügen.

SCHENKER, Oerlikon, 543. 
Leistungen (bzw. Rechtshandlungen) stehen dabei insbesondere Rückzahlungen von Krediten (durch Geld oder auf andere Weise) im Zentrum, sowie die Gewährung von neuen oder Erhöhung von bestehenden Sicherheiten. Solche Handlungen sind potentiell mittels der Absichtspauliana gemäss Art. 288 SchKG und/oder der Überschuldungspauliana gemäss Art. 287 SchKG anfechtbar. Eine Schenkungspauliana gemäss Art. 286 SchKG dürfte in der Regel nicht relevant sein. ${ }^{23}$

\section{a) Überschuldungsanfechtung (Art. 287 SchKG)}

Die Überschuldungsanfechtung trifft bestimmte Rechtshandlungen, mit denen ein überschuldeter Schuldner (Art. 725 OR) einzelne Gläubiger bevorzugt hat. Die Begünstigung besteht regelmässig darin, dass ein Gläubiger vom Schuldner eine Sicherheit oder sogar Befriedigung erhält, auf die er überhaupt nicht oder nicht in der gewählten Art oder doch nicht zu der betreffenden Zeit Anspruch hatte. ${ }^{24}$ Das Gesetz formuliert in Art. 287 Abs. 1 Ziff. 1 bis 3 SchKG drei konkrete Rechtshandlungen, die mittels der Überschuldungsanfechtung anfechtbar sind: i) Nachträgliche Bestellung einer Sicherheit für eine bestehende Verbindlichkeit, ii) Tilgung einer Geldschuld auf ungewohnte Weise, iii) Bezahlung einer noch nicht fälligen Schuld.

Die Überschuldungspauliana setzt voraus, dass der Schuldner eine anfechtbare Handlung innerhalb der einjährigen „période suspecte“ vorgenommen hat. Zudem muss der Schuldner im Zeitpunkt der anfechtbaren Handlung effektiv überschuldet gewesen sein; d.h. die Passiven müssen zu diesem Zeitpunkt die Aktiven überstiegen haben. Eine Anfechtung ist ferner nicht möglich, wenn der Begünstigte beweist, dass er von der Überschuldung des Schuldners keine Kenntnis hatte und davon auch keine Kenntnis hätte haben müssen (Art. 287 Abs. 2 SchKG).

Diese objektiven Tatbestandselemente - Vornahme einer in Art. 287 Abs. 1 Ziff. 1 bis 3 SchKG genannten Rechtshandlung während der einjährigen Verdachtsfrist, und zwar in einem Zeitpunkt, zu dem der Schuldner bereits überschuldet war - sind vom Kläger nachzuweisen. ${ }^{25}$ Subjektive Tatbestandselemente - etwa eine Begünstigungs- oder eine Schädigungsabsicht - sind vom Kläger nicht nachzuweisen und für die Erfüllung des Tatbestandes von Art. 287 SchKG auch nicht erforderlich.

23 Die Schenkungsanfechtung wäre z.B. bei der Insolvenz eines Drittsicherheitsbestellers zu prüfen. Die Thematik der Drittsicherheit wird im Folgenden indes nicht weiter thematisiert (dazu z.B. WOLF, N 220 ff.).

24 Statt aller: AMONN/WALTHER, § 52, N 16.

25 KUKO SchKG-UMBACH-SPAHN/BOSSART, Art. 287, N 1. 
Stillhalteabkommen enthalten regelmässig Bestimmungen, die mit Blick auf eine Überschuldungsanfechtung bei gescheiterter Sanierung problematisch sein könnten. Einerseits beinhalten Stillhalteabkommen weitgehende Informationsrechte des Gläubigers bzw. Informationspflichten des Schuldners. Andererseits werden in Stillhalteabkommen nicht selten anfechtungsgefährdete Handlungen vereinbart, namentlich solche i.S.v. Art. 287 Abs. 1 Ziff. 1 bis 3 SchKG.

\section{aa) Informationsrechte}

Gemäss Art. 287 Abs. 1 SchKG sind gewisse Rechtshandlungen anfechtbar, wenn diese zu einem Zeitpunkt vorgenommen werden, zu dem der Schuldner überschuldet war. Dies aber nur dann, wenn der Begünstigte von der Überschuldung wusste oder von dieser hätte wissen müssen (Art.287 Abs. 2 SchKG).

In Stillhalteabkommen werden häufig weitgehende Informationsrechte zu Gunsten der am Abkommen beteiligten Finanzgläubiger vereinbart. Die Kreditnehmerin wird typischerweise verpflichtet, den Finanzgläubigern regelmässig und detailliert Bericht zu erstatten. In aller Regel umfasst die Berichterstattungspflicht zumindest die periodische Offenlegung der finanziellen Situation des Schuldners, indem er eine aktuelle Bilanz, eine aktuelle Erfolgsrechnung, sowie eine Prognose für die künftige finanzielle Situation (inkl. einer Liquiditätsplanung) vorzulegen hat. Vielfach wird der Schuldner auch zur Offenlegung von weiteren Informationen verpflichtet, beispielsweise Informationen über die Umsetzung des Sanierungskonzepts und die Einhaltung des Budgets, Informationen zu allfälligen Verkaufsbemühungen und Devestitionsplänen, Informationen zum Geschäftsgang und der Auftragslage etc. Der Transfer dieser Informationen vom Schuldner zu den Finanzgläubiger erfolgt vielfach direkt durch den Schuldner. Nicht selten wird von den Finanzgläubigern aber auch verlangt, dass für die Sanierung und Restrukturierung des Schuldners ein (externer) Sanierungsberater eingesetzt wird. Ist dies der Fall, erfolgt der Informationstransfer zum Teil auch durch diesen Sanierungsberater, der als Bestandteil seiner Mandatierung eine Reportingpflicht gegenüber den Finanzgläubigern übernimmt. Schliesslich wird in Stillhalteabkommen teilweise auch vereinbart, dass der Schuldner einen Steuerungsausschuss zur Koordination der Sanierungs- und Restrukturierungsbemühungen schaffen muss, in den die Finanzgläubiger Einsitz nehmen, oder es wird gar vereinbart, dass die Finanzgläubiger an Sitzungen des Verwaltungsrats oder der Geschäftsleitung des Schuldners teilnehmen können. Auch in dieser Konstellation erfolgt ein Informationstransfer vom Schuldner zu den Finanzgläubigern. 
Als Folge dieser Informationsrechte ist u.E. in aller Regel davon auszugehen, dass die Finanzgläubiger über die finanzielle Situation des Schuldners informiert sind, oder informiert sein sollten. Die Kenntnis der Finanzgläubiger um die finanzielle Situation des Schuldners wiederum dürfte dazu führen, dass an einem Stillhalteabkommen teilnehmende Finanzgläubiger auch von einer allfälligen Überschuldung des Schuldners Kenntnis haben oder haben müssten. Als Konsequenz davon ist für die am Stillhalteabkommen teilnehmenden Gläubigern eine Exkulpation über Art. 287 Abs. 2 SchKG in aller Regel nicht möglich.

\section{bb) Handlungen i.S.v. Art. 287 SchKG}

Die in Art. 287 Abs. 1 SchKG genannten Handlungen sind paulianisch anfechtbar, wenn sie zu einem Zeitpunkt vorgenommen werden, zu dem der Schuldner überschuldet war. In Stillhalteabkommen werden vielfach Handlungen vereinbart, die unter Art. 287 Abs. 1 SchKG subsumiert werden können. Da den Finanzgläubigern eine Exkulpation über Art. 287 Abs. 2 SchKG regelmässig nicht gelingen dürfte ${ }^{26}$, ist bei solchen Handlungen besondere Vorsicht geboten.

\section{- (i) Sicherung von Krediten}

Stillhalteabkommen können verschiedene Bestimmungen enthalten, wonach die Kreditnehmerin zur Besicherung von Krediten verpflichtet wird. Einerseits kann vereinbart werden, dass im Gegenzug für das eigentliche Stillhalten bestehende Kredite nachträglich zu besichern, oder dass bestehende Sicherheiten nachträglich zu erhöhen sind. Andererseits wird häufig eine ursprüngliche Besicherung von Überbrückungskrediten vorgesehen. ${ }^{27}$

Unproblematisch ist i.d.R. die ursprüngliche Besicherung von Verbindlichkeiten. Die ursprüngliche Bestellung einer Sicherheit stellt nach ständiger Praxis des Bundesgerichts eine gleichwertige Gegenleistung für die Kreditgewährung $\mathrm{dar}^{28}$, was eine paulianische Anfechtung ausschliesst. ${ }^{29}$ Der Kreditnehmer

26 Dazu Ziff. III.1.a)aa).

27 WOLF, N 330.

28 BGE 126 III 247 E. 3 unter Hinweis auf BGE 53 III 79.

29 In diesem Fall fehlt es i.d.R. an einer Gläubigerschädigung, vgl. oben Ziff. III.1. Unter Umständen kann aber auch die ursprüngliche Besicherung eines Kredits angefochten werden (gemäss Art. 288 SchKG). Dies ist etwa dann der Fall, wenn der Kreditgeber im Zeitpunkt der Kreditgewährung nach den Umständen davon ausgehen musste, dass eine Insolvenz wahrscheinlich nicht vermieden werden kann und der Kredit dazu verwendet wird, ausgewählte ungesicherte Forderungen voll zu decken, was zu einer Schädigung der übrigen Gläubiger führt (BGE 101 III 82 E. 4). 
erhält den Kredit im Gegenzug für die Leistung der Sicherheit. Ebenfalls keine Gläubigerschädigung liegt vor, wenn eine bestehende Sicherheit durch eine andere, gleichwertige Sicherheit ersetzt wird. ${ }^{30}$ Wird im Stillhalteabkommen somit vorgesehen, dass ein neu gewährter Kredit (z.B. ein Überbrückungskredit) zu besichern ist, so ist dieses Vorgehen aus Sicht der Überschuldungsanfechtung nicht zu beanstanden. Nicht zu beanstanden ist sodann, wenn im Stillhalteabkommen vorgesehen wird, eine bestehende Sicherheit gegen eine neue zu ersetzen, vorausgesetzt die neue Sicherheit übersteigt wertmässig die frühere Sicherheit nicht. Ebenfalls unproblematisch ist in aller Regel, wenn im Stillhalteabkommen die Bestellung von Drittsicherheiten vereinbart wird. Ein solcher Vorgang müsste indes im Falle der Insolvenz des Dritten insb. unter dem Titel der Schenkungsanfechtung gemäss Art. 286 SchKG kritisch hinterfragt werden.

Demgegenüber ist gemäss Art. 287 Abs. 1 Ziff. 1 SchKG die Bestellung von Sicherheiten für bereits bestehende Verbindlichkeiten anfechtbar (sofern die Pflicht zur Sicherstellung nicht bereits vorher bestanden hat). ${ }^{31}$ Die nachträgliche Besicherung einer Verbindlichkeit führt regelmässig zu einer Verminderung des Vollstreckungssubstrats und zur Besserstellung des Besicherten gegenüber den nicht besicherten Gläubigern. Das Vorliegen einer Gläubigerschädigung ist deshalb zu bejahen. ${ }^{32}$ In der Lehre wird teilweise postuliert, dass die nachträgliche Besicherung von Verbindlichkeiten nicht zwingend zu einer Gläubigerschädigung führt, sondern als Austausch von gleichwertigen Leistungen angesehen werden kann, was eine paulianische Anfechtung ausschliesst. ${ }^{33}$ Diesem Ansatz ist u.E. zuzustimmen. Wird durch die nachträgliche Bestellung von Sicherheiten die Situation für sämtliche Gläubiger verbessert, so kann darin keine Gläubigerschädigung erkannt werden, was die paulianische Anfechtung ausschliesst. Soweit ersichtlich besteht indes keine diese Auffassung stützende Judikatur. Zudem dürfte es zum Zeitpunkt der nach-

30

31 Gemäss GLANZMANN, 284, gilt als nachträgliche Besicherung eines bestehenden Kredits auch die Besicherung eines neuen Kredites, der dazu verwendet wird, eine bereits bestehende Verbindlichkeit des Schuldners gegenüber dem gleichen Gläubiger zu tilgen.

gl. Urteil des Bundesgerichts 5A_862/2010 vom 16. Februar 2012 E. 2: „Eine Schädigung anderer Gläubiger tritt auch ein, wenn nachträglich Sicherheiten bestellt werden, und zwar selbst dann, wenn sich der Schuldner zu deren Bestellung bereits bei der Kreditaufnahme verpflichtet hat." Anzumerken ist, dass sich die genannte Erwägung auf Art. 288 SchKG bezieht, also kein Widerspruch zu Art. 287 Abs. 1 Ziff. 1 SchKG, zweiter Satzteil darstellt. nachträgliche Besicherung auf die Fälligstellung des Kredits verzichtet oder noch nicht benützte Kreditlinien offenhält und damit das Eintreten eines Event of Default verhindert. Vgl. auch GLANZMANN, 271 und 267. 
träglichen Bestellung von Sicherheiten kaum je mit Gewissheit absehbar sein, dass durch die nachträgliche Sicherungsbestellung tatsächlich eine Besserstellung sämtlicher Gläubiger erreicht wird. Aus Gründen der Diligenz ist deshalb davon auszugehen, dass die in einem Stillhalteabkommen vorgesehene nachträgliche Besicherung von bestehenden Verbindlichkeiten, oder der Ersatz einer bestehenden Sicherheit durch eine wertmässig höhere Sicherheit paulianisch anfechtbar ist und dem am Stillhalteabkommen teilnehmenden Finanzgläubiger keinen genügenden Schutz bietet.

- (ii) Unübliche Tilgung

Stillhalteabkommen sehen regelmässig die Tilgung von finanziellen Verbindlichkeiten des Schuldners vor. Sofern es sich um die Tilgung von fälligen Verbindlichkeiten auf „übliche“ Weise handelt, ist diese aus Sicht der Überschuldungsanfechtung grundsätzlich nicht zu beanstanden. ${ }^{34}$

Demgegenüber ist die Tilgung einer Geldschuld durch „unübliche“ Mittel mittels der Überschuldungsanfechtung anfechtbar, sofern der Schuldner im Zeitpunkt der Tilgung überschuldet war und der Finanzgläubiger davon Kenntnis hatte. ${ }^{35}$ Dies selbstverständlich nur dann, wenn dieser Vorgang innert der einjährigen „période suspecte" erfolgte und ein Insolvenzfall (d.h. Konkurs oder Nachlassvertrag mit Vermögensabtretung) vorliegt.

Die Tilgung einer Geldschuld gilt als ungewöhnlich, wenn sie auf andere Weise als durch Barschaft oder durch anderweitige übliche Zahlungsmittel erfolgt (Art. 287 Abs. 1 Ziff. 2 SchKG). Die Ungewöhnlichkeit der Tilgungshandlung bestimmt sich anhand der Orts- und Branchenüblichkeit sowie den vorbestehenden Gepflogenheiten der Parteien. ${ }^{36}$ Als unüblich gilt regelmässig die Tilgung von Verbindlichkeiten durch Übertragung von Aktiven (Debt/AssetSwap) und die Anweisung an einen Schuldner des Kreditschuldners zur direkten Zahlung an den (Kredit-) Gläubiger. ${ }^{37}$ Die Abtretung von Forderungen zu Tilgungszwecken gilt i.d.R. nur dann als übliches Zahlungsmittel, wenn eine entsprechende Branchenüblichkeit nachgewiesen werden kann. Eine Abtretung zahlungshalber hat üblicherweise nicht den Charakter einer ordentlichen Tilgung. ${ }^{38}$ Demgegenüber gilt die Tilgung einer Verbindlichkeit durch Verrechnung regelmässig als gewöhnliche Tilgung. ${ }^{39}$

\footnotetext{
34 Bei gegebenen Voraussetzungen sind diese Rückzahlungen jedoch unter dem Titel der Absichtspauliana (Art. 288 SchKG) problematisch.

35 Dazu oben Ziff. III.1.a)aa).

36 KUKO SchKG-Umbach-SpaHn/Bossart, Art. 287, N 5.

37 WOLF, N 167.

38 KUKO SchKG-UMBACH-SPAHN/BOSSART, Art. 287, N 5.

39 Wolf, N 167 unter Hinweis auf BSK SchKG-StaEHELIN, Art. 287, N 9.
} 
Insbesondere infolge der regelmässigen Unmöglichkeit für an Stillhalteabkommen beteiligte Finanzgläubiger, sich gemäss Art. 287 Abs. 2 SchKG auf die Unkenntnis einer Überschuldung des Schuldners berufen zu können, sollte falls möglich - auf die Vereinbarung von Tilgungen durch unübliche Mittel ganz verzichtet werden. Dies jedenfalls dann, wenn die Überschuldung des Schuldners nicht vollends ausgeschlossen werden kann.

Analog den Ausführungen zur Sicherung von Krediten ${ }^{40}$ wird in der Lehre teilweise postuliert, dass auch eine unübliche Tilgung nicht unbedingt zu einer Gläubigerschädigung führt, sondern diese als gleichwertige Gegenleistung für das Stillhalten angesehen werden kann. ${ }^{41}$ Dieser Lehrmeinung ist zuzustimmen. Mangels einschlägiger (und bestätigender) Rechtsprechung ist aber aus Gründen der Diligenz davon auszugehen, dass die unübliche Tilgung von Schulden zu einer Schädigung der Gläubiger führt und entsprechende Rechtshandlungen somit gemäss Art. 287 SchKG anfechtbar sind.

- (iii) Vorfällige Tilgung

Gemäss Art. 287 Abs. 1 Ziff. 3 SchKG ist die Zahlung einer nicht verfallenen Schuld unter dem Titel der Überschuldungsanfechtung anfechtbar.

U.E. kommt dieser Bestimmung im Kontext von Stillhalteabkommen keine grosse Bedeutung zu. Regelmässig liegt zum Zeitpunkt des Abschlusses des Stillhalteabkommens bereits ein Event of Default (eventuell in Verbindung mit einer Cross Default Situation) vor, und sämtliche Verpflichtungen gegenüber den Finanzgläubigern sind infolge der vertraglichen Abreden mit dem Gläubiger ohnehin bereits fällig geworden. ${ }^{42}$

\section{b) Absichtsanfechtung (Art. 288 SchKG)}

Gemäss Art. 288 SchKG sind sämtliche Rechtshandlungen anfechtbar, die der Schuldner innerhalb einer "période suspecte“ von 5 Jahren in der erkennbaren Absicht vorgenommen hat, seine Gläubiger zu benachteiligen oder einzelne Gläubiger zum Nachteil anderer zu begünstigen.

Objektive Voraussetzung der Anfechtungsklage ist, dass die angefochtene Handlung die Gläubiger schädigt, indem sie das Vollstreckungsergebnis oder ihren Anteil daran vermindert, oder ihre Stellung im Vollstreckungsverfahren sonst wie verschlechtert. Dabei genügt bereits die Schädigung eines einzelnen

40 Vorstehend, Ziff. III.1.a)bb).

41 WOLF, N 160 und 315.

42 Vgl. Auch Fischer R., 54, wonach kein Fall von Art. 287 Abs. 1 Ziff. 3 SchKG vorliegt, wenn vom Kreditgeber im Einklang mit den vertraglichen Bestimmungen eine vorzeitige Fälligkeit herbeigeführt wird. 
Gläubigers. Keine Gläubigerschädigung hat eine Rechtshandlung zur Folge, wenn eine gleichwertige Gegenleistung erhalten wird, es sei denn der Schuldner habe mit dem Geschäft den Zweck verfolgt, über seine letzten Aktiven zum Schaden der Gläubiger zu verfügen. ${ }^{43}$

Als subjektives Tatbestandsmerkmal ist erforderlich, dass der Schuldner mit der Absicht der Gläubigerschädigung handelte. Dabei genügt es, wenn die Schädigung eine mögliche Folge der Handlung ist und der Schuldner diese eventualvorsätzlich in Kauf nimmt; blosse Fahrlässigkeit ist hingegen nicht ausreichend. ${ }^{44}$ Als weiteres, kumulativ erforderliches subjektives Tatbestandselement ist vorausgesetzt, dass die Schädigungsabsicht des Schuldners für den begünstigten Dritten im Zeitpunkt des anfechtbaren Rechtsgeschäfts erkennbar war. Dieser musste sie (die Schädigungsabsicht) bei Einhaltung der gebotenen Sorgfalt und Aufmerksamkeit erkennen können, wobei Fahrlässigkeit hier ausreichend ist. ${ }^{45}$

Diese objektiven und subjektiven Tatbestandsmerkmale - Gläubigerschädigung, Schädigungsabsicht, Erkennbarkeit der Schädigungsabsicht - sind vom Kläger nachzuweisen.

In Stillhalteabkommen finden sich oft Vereinbarungen, die potentiell als gläubigerschädigend i.S.v. Art. 288 SchKG qualifiziert werden könnten. Sowohl bei der Formulierung des Stillhalteabkommens, wie auch bei der geschäftlichen Handhabung des Abkommens ist deshalb Vorsicht geboten.

\section{aa) Gläubigerschädigung}

In Stillhalteabkommen wird in aller Regel festgehalten, dass bzw. wann und wie der Schuldner seinen Verpflichtungen gegenüber den am Stillhalteabkommen teilnehmenden Finanzgläubigern nachzukommen hat, namentlich wie und wann die gewährten Kredite zurückzuführen sind. Dabei sind die Grundsätze zur Gläubigerschädigung gemäss Art. 288 SchKG zu beachten.

Bei Darlehen gilt die Rückzahlung des Darlehensbetrags nicht als eine (gleichwertige) Gegenleistung für die Hingabe des Darlehensbetrags, sondern als Erfüllung der mit der Darlehensaufnahme eingegangenen Verpflichtung zur

\footnotetext{
43 KUKO SchKG-UMBACH-SPAHN/BOSSART, Art. 288, N 3.

44 KUKO SchKG-UMBACH-SPAHN/BOSSART, Art. 288, N 9.
}

45 KUKO SchKG-UMBACH-SPAHN/BOSSART, Art. 288, N 15. 
späteren Darlehensrückzahlung. Die Rückzahlung gilt mit anderen Worten als Gläubigerschädigung i.S.v. Art. 288 SchKG und ist deshalb - sofern die übrigen Voraussetzungen erfüllt sind - anfechtbar. ${ }^{46}$

Keine Gläubigerschädigung liegt vor, wenn ein (ursprünglich und vollumfänglich) gesichertes Darlehen zurückbezahlt wird. Dadurch erfolgt keine (ungerechtfertigte) Ungleichbehandlung der Gläubiger. Die Rückzahlung eines gesicherten Darlehens ist deshalb paulianisch nicht anfechtbar. Wird die Sicherheit für das Darlehen demgegenüber erst nachträglich bestellt, so ist in der Bestellung der Sicherheit (und auch in deren späterer Honorierung) eine gläubigerschädigende Handlung zu sehen. Sind die übrigen Voraussetzungen erfüllt, kann die nachträgliche Besicherung eines Kredits bzw. die Honorierung einer nachträglich bestellten Sicherheit paulianisch angefochten werden.

Anders als bei der fristgerechten Rückzahlung des Darlehens gilt die fristgerechte Zahlung fälliger Zinsen als gleichwertige Gegenleistung für die fortdauernde Überlassung des früher bereits bezahlten Darlehensbetrages. ${ }^{47}$ Die Zahlung von Darlehenszinsen stellt deshalb i.d.R. keine Gläubigerschädigung dar, sondern gilt als Entgelt für die Aufrechterhaltung des Darlehens. Eine paulianische Anfechtung solcher Zinszahlungen ist deswegen ausgeschlossen. Die Argumentation, der Zins sei als Entgelt für die Aufrechterhaltung des Darlehens zu verstehen, verfängt aber dann nicht mehr, wenn die Zinsen nicht periodisch über die Laufzeit des Darlehens bezahlt werden, sondern - beispielsweise - zusammen mit dem Kapital bei dessen Fälligkeit. ${ }^{48}$ Die Gewährung einer Stundung für die Darlehenszinsen dürfte deshalb regelmässig dazu führen, dass die Zinsen nicht mehr als gleichwertige Gegenleistung für die fortdauernde Überlassung des Darlehensbetrags qualifiziert werden. Schliesslich müssen sie in diesem Fall nicht mehr bezahlt werden, um die fortlaufende Überlassung des Darlehensbetrags zu gewähren. Analog zur Rückzahlung des Darlehens hat dies zur Folge, dass auch die Bezahlung der Zinsen als gläubigerschädigende Handlung anzusehen ist. Dies ist im Zusammenhang mit Stillhalteabkommen von besonderer Relevanz, wird vom Stillhalten (bzw. von der gewährten Stundung) doch regelmässig auch der Darlehenszins mitumfasst. Als Folge ist die Bezahlung der Darlehenszinsen nicht mehr als gleichwertige Gegenleistung für die Aufrechterhaltung des Darlehens anzusehen, und Zinszahlungen werden potentiell paulianisch anfechtbar.

BSK SchKG-BAUER, Art. 288, N 13 mit Hinweis auf die reiche Praxis des Bundesgerichts. Urteil des Bundesgerichts 5A_750/2008 vom 24. Februar 2010 E. 4.4.; WOLF, N 318 ff; BSK SchKG-BAUER, Art. 288, N 13a mit (berechtigter) Kritik an dieser Rechtsprechung. BSK SchKG-BAUER, Art. 288, N 13b. Gleiches gilt, wenn ein Einmalzins - zahlbar am Ende der Laufzeit des Darlehens - vereinbart wurde. 


\section{bb) Schädigungsabsicht und Erkennbarkeit der Schädigungsabsicht}

Subjektive Voraussetzung einer Absichtsanfechtung i.S.v. Art. 288 SchKG ist das Vorliegen einer Schädigungsabsicht beim Schuldner und deren Erkennbarkeit für den begünstigten Gläubiger.

Die Schädigungsabsicht i.S.v. Art. 288 SchKG liegt vor, wenn der Schuldner voraussehen konnte und musste, dass die angefochtene Handlung Gläubiger benachteiligt oder einzelne Gläubiger gegenüber anderen bevorzugt. Dabei genügt es, wenn die Schädigung eine mögliche Folge der Handlung ist und der Schuldner sie im Sinne eines Eventualvorsatzes in Kauf nimmt. ${ }^{49}$ Als innere Tatsache kann die Schädigungsabsicht in aller Regel nicht direkt bewiesen werden. Es darf deshalb auf Indizien abgestellt werden, welche sich aus dem äusseren Verhalten des Schuldners und den äusseren Gegebenheiten, die auf ihn eingewirkt haben, ergeben. War sich der Schuldner seinen finanziellen Schwierigkeiten bewusst, so besteht gemäss Bundesgericht ein gewichtiges Indiz für eine Schädigungsabsicht. ${ }^{50}$ Je gravierender und offensichtlicher die Finanzprobleme sind, desto wahrscheinlicher ist, dass der Schuldner diese auch erkannte. ${ }^{51}$ Wird ein Stillhalteabkommen abgeschlossen, so ist davon auszugehen, dass sich der Schuldner seinen finanziellen Schwierigkeiten bewusst ist; ansonsten hätte kein Stillhalteabkommen abgeschlossen werden müssen. Angesichts der bundesgerichtlichen Rechtsprechung besteht deshalb ein erhebliches Risiko, dass bei Rückzahlungen, die im Zusammenhang mit Stillhalteabkommen vereinbart und geleistet werden, davon ausgegangen wird, dass die Zahlungen zumindest unter Inkaufnahme einer Gläubigerschädigung erfolgten. Diese Schlussfolgerung wird in der Lehre teilweise kritisiert. ${ }^{52}$ Der Kritik ist insofern zuzustimmen, als eine Zahlung in Kenntnis um die eigenen finanziellen Schwierigkeiten nicht den Nachweis für eine Schädigungsabsicht darstellt. ${ }^{53}$ Es muss dem Schuldner möglich sein, aufzuzeigen, dass eine Zahlung ohne Schädigungsabsicht erfolgt ist, obwohl ein Indiz für die Schädi-

49 KUKO SchKG-UMBACH-SPAHN/BOSSART, Art. 288, N 9.

$50 \quad$ BGE 134 III 452 E. 7.4: „In Anbetracht des Wissens um die schlechte finanzielle Lage muss davon ausgegangen werden, dass die SAirGroup zumindest in Kauf genommen hat, durch ihre drei Zahlungen an die Beschwerdegegnerin könnten andere Gläubiger geschädigt werden." Bestätigt im Urteil des Bundesgerichts 5A_116/2009 vom 28. September 2009 E. 6.3; Urteil des Bundesgerichts 5A_358/2005 vom 3. August 2010 und Urteil des Bundesgerichts 5A_473/2009 vom 3. August 2010 E. 2.2.3.

$51 \quad$ KRIESI, 222.

52 Dazu Wolf, N 173; vgl. auch KUKO SchKG-UMBACH-SPAHN/BOSSART, Art. 288, N 13. Zum Sanierungsdarlehen: Nachfolgend, Ziff. III.1.b)cc).

53 Kenntnis um die finanziellen Schwierigkeiten ist ein Indiz für die Schädigungsabsicht (und deren Erkennbarkeit), kein Nachweis. 
gungsabsicht vorliegt. Relevant ist insbesondere (auch), ob im Zeitpunkt der anfechtbaren Handlung berechtigterweise mit der Sanierung des Schuldners gerechnet werden durfte. ${ }^{54}$

Die Schädigungsabsicht des Schuldners muss für den Begünstigten im Zeitpunkt des anfechtbaren Rechtsgeschäfts erkennbar sein. Dieser muss die Schädigungsabsicht bei Einhaltung der gebotenen Sorgfalt und Aufmerksamkeit - mithin ohne Fahrlässigkeit - erkennen können. ${ }^{55}$ Es genügt also, wenn der Begünstigte bei Anwendung der nach den Umständen gebotenen und zumutbaren Sorgfalt und Aufmerksamkeit hätte erkennen können und müssen, dass als Folge der angefochtenen Handlung möglicherweise eine Gläubigerschädigung eintritt. Das Bundesgericht behilft sich beim Kriterium der Erkennbarkeit der Schädigungsabsicht mit der Rechtsfigur der Erkundigungsobliegenheit des durch die fragliche Rechtshandlung Begünstigten. Wenn deutliche Anzeichen dafür sprechen, dass eine Schädigung beabsichtigt ist, darf vom Begünstigten eine sorgfältige Prüfung verlangt werden, ob die (Schädigungs-) Absicht wirklich besteht oder nicht. ${ }^{56}$ Bei Stillhalteabkommen ist zu berücksichtigen, dass die von den Finanzgläubigern jeweils ausbedungenen Informationsrechte - sofern diese nicht ohnehin bereits infolge der Regelungen in den zu Grunde liegenden Kreditverträgen bestehen - in aller Regel dazu führen, dass die Finanzgläubiger Kenntnis um die finanziellen Schwierigkeiten des Schuldners haben. Den Kreditgebern erwachsen dadurch weitergehende Informationspflichten und die Erkennbarkeit der Schädigungsabsicht dürfte vielfach bejaht werden.

Wird eine nahestehende Person begünstigt, so trägt der Nahestehende die Beweislast dafür, dass er die Schädigungsabsicht des Schuldners nicht erkennen konnte (Art. 288 Abs. 2 SchKG). Eine Definition der nahestehenden Person findet sich im Gesetz nicht. Es wurde bewusst eine offene, generalklauselartige Umschreibung verwendet, um der Rechtsprechung die Möglichkeit für eine entsprechende Differenzierung und einzelfallgerechte Beurteilung zu ermöglichen. Nach der hier vertretenen Auffassung gilt z.B. die Hausbank nicht als nahestehende Person. ${ }^{57}$ Dasselbe hat u.E. auch für die Teilnehmer an einem Stillhalteabkommen zu gelten. Auch diese sind nicht als nahestehende Person i.S.v. Art. 288 Abs. 2 SchKG zu qualifizieren, nur weil sie sich an einem Stillhalteabkommen beteiligen.

\footnotetext{
54 Dazu Wolf, N 173 m.w.H.

55 KUKO SchKG-UMBACH-SPAHN/BOSSART, Art. 288, N 15.

56 WOLF, N 178.

57 Dazu: BernheIM/GeIGER, $13 \mathrm{f}$.
} 


\section{cc) Sanierungsdarlehen}

Beim Darlehen gilt die Rückzahlung des Darlehensbetrags bekanntlich nicht als eine (gleichwertige) Gegenleistung für die Hingabe des Darlehensbetrags. Die Rückzahlung des Darlehens ist deshalb grundsätzlich paulianisch anfechtbar. ${ }^{58}$ Eine Ausnahme bildet das sogenannte Sanierungsdarlehen, welches privilegiert behandelt wird. ${ }^{59}$ Gewährung wie Rückzahlung eines Darlehens, welches dem Schuldner in bedrängter Lage zum Zweck der Sanierung gewährt wurde, sind als Einheit anzusehen, aus der sich unter dem Gesichtspunkt der Anfechtung einerseits die Gleichwertigkeit von Leistung (Darlehensauszahlung) und Gegenleistung (Darlehensrückzahlung) ergibt, bei der andererseits auch die Schädigungsabsicht entfällt. ${ }^{60}$ Entsprechend ist die Rückzahlung eines Sanierungsdarlehens paulianisch nicht anfechtbar. ${ }^{61}$ Die Rechtsfigur des Sanierungsdarlehens ist in der bundesgerichtlichen Rechtsprechung anerkannt. ${ }^{62}$

Die Qualifikation eines Darlehens als Sanierungsdarlehen knüpft an drei Voraussetzungen. Der sich in wirtschaftlichen Schwierigkeiten befindliche Schuldner muss sich um die Sanierung ${ }^{63}$ bemühen. $^{64}$ Sodann müssen die Sanierungsbemühungen zum Zeitpunkt der Darlehensgewährung als erfolgs-

58 Dazu oben, Ziff. III.1.b)aa).

59 Allgemein zum Sanierungsdarlehen und dessen Behandlung im Konkurs: RотH, Krisendarlehen, $41 \mathrm{ff}$.

60 BSK SchKG-BAUER, Art. 288, N 13. Zur Gleichwertigkeit von Leistung und Gegenleistung: GLANZMAnN, $267 \mathrm{f}$.

61 Entweder weil die Schädigungsabsicht bzw. deren Erkennbarkeit nicht gegeben ist (Urteil des Bundesgerichts 5A_671/2018 vom 8. September 2020; BGE 134 III 452) oder weil die Darlehensrückzahlung nicht als Gläubigerschädigung qualifiziert (BGE 137 III 268).

62 Urteil des Bundesgerichts 5A_671/2018 vom 8. September 2020, BGE 137 III 268, BGE 134 III 452, Urteil des Bundesgerichts 5A_116/2009 vom 28. September 2009, Urteil des Bundesgerichts 5A_386/2008 vom 6. April 2009, BGE 99 III 27. Demgegenüber scheint die Exekutive davon auszugehen, dass die Rechtsfigur des Sanierungsdarlehens unnötig ist. Sowohl im Zuge der Revision des Sanierungsrechts, wie auch bei der aktuellen Aktienrechtsrevision wurde der Bedarf einer gesetzlichen Regelung zum Sanierungsdarlehen verneint. Vgl. dazu: VANDEBROEK, N 3.

63 Zum relevanten Begriff der Sanierung, vgl. Urteil des Bundesgerichts 5A_671/2018 vom 8. September 2020 E.4.1: Der anfechtungsrelevante Sanierungsbegriff stimmt nicht überein mit dem bilanzrechtlichen Sanierungsbegriff (Art. 725 OR). Bei der Frage ob ein Darlehen der „Sanierung“ dient und nicht anfechtbar ist, ist auf die Optik der Gläubiger abzustellen.

64 Urteil des Bundesgerichts 5A_671/2018 vom 8. September 2020 E. 3.5.2; BGE 134 III 452 E. 5.5. 
versprechend erscheinen. ${ }^{65}$ Schliesslich muss das Darlehen zum Zwecke der Sanierung gewährt worden sein. ${ }^{66}$ Es bedarf mit anderen Worten eines „Entgegenkommens" bzw. einer „Sonderleistung" des Kreditgebers; ein Verhalten wie ein „gewöhnlicher" Kreditgeber ist nicht ausreichend. ${ }^{67}$

Sofern die genannten Voraussetzungen zur Qualifikation eines Darlehens als Sanierungsdarlehen erfüllt sind, können u.E. Überbrückungskredite, die im Zuge des Abschlusses eines Stillhalteabkommens vereinbart worden sind als Sanierungsdarlehen betrachtet und zurückbezahlt werden. Die paulianische Anfechtung der Rückzahlung ist dann ausgeschlossen. In der Praxis problematisch ist allerdings, dass die Kriterien zur Qualifikation als Sanierungsdarlehen zu wenig exakt und nicht griffig definiert sind. Es besteht deshalb stets die latente Gefahr, dass ein Darlehen von einem Gericht nicht als Sanierungsdarlehen qualifiziert wird und die Rückzahlung des Darlehens entsprechend problematisch wird, was sich negativ auf die Bereitschaft zur Kreditgewährung in Krisensituationen auswirkt. ${ }^{68}$ Die Problematik könnte durch die Gewährung von (ursprünglich) gesicherten Überbrückungsdarlehen adressiert werden. In der finanziellen Krisensituation dürfte es dem Kreditschuldner aber regelmässig nicht möglich sein, die geforderten Sicherheiten beizubringen.

Ebenfalls denkbar ist, dass ein ursprünglich „normales“ Darlehen infolge des Abschlusses eines Stillhalteabkommens in ein Sanierungsdarlehen umqualifi-

65 Urteil des Bundesgerichts 5A_671/2018 vom 8. September 2020 E. 3.5.2; BGE 134 III 452 E. 5.3. Die bundesgerichtliche Rechtsprechung zur günstigen Sanierungsprognose ist indes nicht einheitlich. Ein Überblick findet sich in: KRIESI, 229 f. Ausdrücklich verworfen wurde in BGE 134 III 452 E. 5.1 die Auffassung des Handelsgerichts des Kantons Zürich, wonach die Absichtsanfechtung in einer Sanierungsphase die Erkenntnis oder beim Schuldner das Erkennen müssen und beim Gläubiger das Erkennen sollen voraussetzt, dass die Sanierung mit hoher Wahrscheinlichkeit nicht mehr möglich ist.

66 Urteil des Bundesgerichts 5A_671/2018 vom 8. September 2020 E. 3.5.2; BGE 134 III 452 E. 6; Urteil des Bundesgerichts 5A_116/2009 vom 28. September 2009 E. 6.1; Urteil des Bundesgerichts 5A_386/2008 vom 6. April 2009 E. 4.1.

67 Das Geld darf also nicht in der blossen Absicht der kurzfristigen Anlage zu einem hohen Zins hingegeben werden. Gemäss BGE 134 III 452 E. 6.1 kann der Beitrag zur Sanierung auch in der blossen Verlängerung eines früher gewährten Darlehens bestehen, wenn der Darlehensgeber aufgrund der Kreditvereinbarung berechtigt ist, das Darlehen jederzeit nach freiem Ermessen sofort zur Rückzahlung fällig zu stellen. Vgl. auch Urteil des Bundesgerichts 5A_671/2018 vom 8. September 2020 E. 4.3, Urteil des Bundesgerichts 5A_116/2009 vom 28. September 2009 E. 6.1 und Urteil des Bundesgerichts 5A_386/2008 vom 6. April 2009 E. 4.1. 
ziert wird mit den entsprechenden Privilegien bei der Darlehensrückzahlung. In seinem Entscheid vom 6. Juni 2018 äusserte sich das Appellationsgericht Basel-Stadt wie folgt ${ }^{69}$ :

„Es ist somit nicht ausgeschlossen, dass ein vorbestehendes Darlehen in ein Sanierungsdarlehen umgewandelt werden kann, namentlich durch Verlängerung eines auslaufenden Kredits. Dies setzt einen subjektiven Sanierungswillen voraus. Ein solcher kann sich aus entsprechenden Parteiaussagen oder äusseren Umständen ergeben, die objektiv auf einen entsprechenden Willen schliessen lassen, wie etwa eine Zweckvereinbarung zwischen Darleiher und Borger (vgl. Urteil des Bundesgerichts 5A_116/2009 vom 28. September 2009 E. 6.1, Urteil des Bundesgerichts 5A_386/2008 vom 6. April 2009 E. 4.1). Wenn die Verlängerung des Darlehens lediglich der bisherigen Praxis oder der Kreditpolitik des Darlehensgebers entspricht, ist dieses nicht als Sanierungsdarlehen zu qualifizieren (vgl. BGE 134 III 452 E. 6.1 und E. 6.2)."

Dieser Entscheid des Appellationsgericht Basel-Stadt wurde vom Bundesgericht mit Urteil 5A_671/2018 vom 8. September 2020 bestätigt. Das Bundesgericht hat festgehalten, dass ein bestehendes Darlehen in ein Sanierungsdarlehen gewandelt werden kann und bestätigt, dass auch die Verlängerung eines früher gewährten Darlehens zur Sanierung beitragen und als Sanierungsdarlehen gelten kann, sofern der Sanierungswille (z.B. durch eine Sanierungsvereinbarung) zum Ausdruck kommt und sich das Verhalten (wie durch Sonderleistungen, eigentliches Entgegenkommen, direkte Unterstützung) von gewöhnlichen Kreditgebern unterscheidet. ${ }^{70}$

\section{Aktienrechtliche Verantwortlichkeit}

Das Aktienrecht enthält in den Art. 752 bis 760 OR Bestimmungen über die persönliche Haftbarkeit für Fehlverhalten eines Personenkreises, in dessen Zentrum die Mitglieder des Verwaltungsrats und der Geschäftsleitung sowie die Revisionsstelle stehen, der aber auch die mit der Gründung und der Emission von Prospekten befassten Personen umfasst. Die Voraussetzungen der aktienrechtlichen Verantwortlichkeit entsprechen denjenigen des allgemeinen Haftpflichtrechts: Nebst der Aktiv- und Passivlegitimation muss ein Schaden vorliegen, welcher natürlich und adäquat kausal durch eine schuldhaft begangene Pflichtverletzung verursacht wurde. ${ }^{71}$

69 Urteil des Appellationsgericht des Kantons Basel-Stadt, ZB.2017.34 vom 6. Juni 2018 E. 4.2.1.

70 Urteil des Bundesgerichts 5A_671/2018 vom 8. September 2020 E. 3.5.2.

71 Statt aller: MEIER-HAYOZ/ForSTMOSER/SETHE, § 16, N $784 \mathrm{f}$. 
Im Kontext von Stillhalteabkommen ist die Frage relevant, inwiefern sich die an einem Stillhalteabkommen teilnehmenden Finanzgläubiger dem Risiko einer aktienrechtlichen Verantwortlichkeit aussetzen, sofern die Sanierung des Schuldners misslingt und dieser insolvent wird. ${ }^{72}$

a) Faktische Organschaft

\section{aa) Allgemeines}

Gemäss Art. 754 OR sind alle mit der Geschäftsführung befassten Personen für den Schaden verantwortlich, den sie durch absichtliche oder fahrlässige Verletzung ihrer Pflichten verursachen. Mit dieser Formulierung von Art. 754 OR wird zum Ausdruck gebracht, dass nicht nur Verwaltungsratsmitglieder und weitere Organe im formellen Sinne - wie Mitglieder der Direktion, Geschäftsoder Konzernleitung - erfasst werden sollen, sondern alle Personen, die tatsächlich Organfunktionen erfüllen und so die Willensbildung der Gesellschaft massgebend und in organtypischer Weise mitbestimmen, sei es, dass ihnen solche zugewiesen worden sind, sei es, dass sie diese auch einfach tatsächlich ausüben. Im ersten Fall spricht man von einem materiellen Organ, im zweiten von einem faktischen Organ. ${ }^{73}$ Faktische Organe sind mit anderen Worten Personen, die tatsächlich Organen vorbehaltene Entscheide treffen oder die eigentliche Geschäftsführung besorgen und so die Willensbildung der Gesellschaft massgebend mitbestimmen. ${ }^{74}$

In Judikatur und Literatur wurden verschiedene Kriterien entwickelt, die für die Qualifikation als faktisches Organ relevant sein können. Erforderlich ist, dass jemand derart auf Gesellschaftsentscheide einwirkt, wie dies üblicherweise und typischerweise durch Organe im formellen Sinn geschieht. Es bedarf der Einflussnahme auf Bereiche, die zur Verwaltung bzw. Geschäftsführung gehören und normalerweise dem Verwaltungsrat bzw. der Geschäftsleitung vorbehalten sind. Zudem muss die Art der Einwirkung organtypisch sein, d.h. die betroffene Person muss so tun, wie wenn sie Organ wäre. In einem jüngeren Entscheid ${ }^{75}$ hielt das Bundesgericht fest, dass für die Qualifikation als faktisches Organ erforderlich ist, dass eine andauernde Befugnis besteht, über die Erledigung des Tagesgeschäfts hinausgehende Entscheide zu fassen. Die

72

Wobei - analog zur paulianischen Anfechtung - die aktienrechtliche Verantwortlichkeit nur im Falle eines Konkurses und eines Nachlassvertrags mit Vermögensabtretung einschlägig sein dürfte. Dazu oben, Ziff. III.

73

74

75 
Entscheidkompetenz des faktischen Organs muss selbständig und unabhängig erscheinen. Blosse Hilfe bei der Entscheidung - selbst bei starkem Einfluss genügt nicht, um den Status des faktischen Organs zu erlangen ${ }^{76}$. In der Literatur wurden die folgenden Kriterien zur Qualifikation als faktisches Organ entwickelt: ${ }^{77}$

- (1) Person: Weite Definition, als faktische Organe können insbesondere auch juristische Personen in Frage kommen; und

- (2a) Organtypische Funktion: Bloss generelle Einflussnahme auf die Entscheidfindung genügt nicht; erforderlich sind einerseits organtypische Kompetenzen und andererseits das tatsächliche Wahrnehmen organtypischer Funktionen (,handeln wie wenn er Organ wäre“ $)^{78}$; oder

- (2b) Besorgung der Geschäftsführung: D.h. Vertreten der bzw. Auftreten als die Gesellschaft gegen aussen; und

- (3) Massgebliche Mitbestimmung bei der Willensbildung: Massgebliche Mitbestimmung bei unternehmenspolitischen und -leitenden Entscheiden (insb. Willensbildung im Verwaltungsrat und in der Geschäftsleitung).

Verneint wird die Organstellung desjenigen, der nur insoweit auf die Geschicke einer Gesellschaft Einfluss nimmt, als dies nach objektiver Betrachtung zur Wahrung seiner eigenen Interessen nötig ist. ${ }^{79}$

\section{bb) Relevanz bei Stillhalteabkommen}

In Stillhalteabkommen finden sich teilweise Bestimmungen, die dazu führen können, dass der teilnehmende Finanzgläubiger als faktisches Organ des Kreditnehmers erscheint. ${ }^{80}$ Finanzgläubiger werden in der Regel aber nicht zum faktischen Organ des Kreditnehmers bzw. Schuldners, sofern sie sich auf die eigentliche Rolle als Kreditgläubiger beschränken.

76 BGE 136 III 14 E. 2.4.; GLANZMANN/WOLF, 336.

77 KunZ, 179.

78 Beispielsweise regelmässige Teilnahme an Verwaltungsratssitzungen mit gleichwertigem Stimmrecht; Einladung zu sämtlichen Verwaltungsratssitzungen; regelmässiges Unterbreiten von Verwaltungsratsbeschlüssen (mit Vetorecht); gemeinsames Auftreten gegenüber Dritten (als Organ).

79 FORSTMOSER, N 667. Nicht Organ, sondern schuldvertraglicher Partner ist daher, wer in Verhandlungen über die Gewährung oder Fortführung von Krediten einer Gesellschaft Auflagen macht. Vgl. auch BGE 136 III 14 E. 2.4 und Urteil des Bundesgerichts 4A_147/2014 vom 19. November 2014 E. 6.3.

80 Dazu auch: WoLF, N 385 ff. 
Hinsichtlich einer Qualifikation als faktisches Organ unproblematisch erscheinen uns das eigentliche Stillhalten ${ }^{81}$, die Verpflichtung zur Leistung von Sanierungsbeiträgen, die Vereinbarung von Auflagen für die Kreditgewährung und die Vereinbarung von Informationspflichten. Damit nimmt der Finanzgläubiger keinen organtypischen Einfluss auf die Gesellschaft und besorgt auch nicht deren Geschäftsführung. Die Vereinbarung von Auflagen für die Kreditgewährung und von Informationspflichten erfolgt sodann zur Wahrung der eigenen Interessen des Finanzgläubigers.

In der Regel unproblematisch im Hinblick auf die Qualifikation als faktisches Organ ist auch die Verpflichtung des Schuldners zur Einleitung von Sanierungs- oder Restrukturierungsmassnahmen, die Verpflichtung zum Beizug eines Sanierungsberaters sowie die Vereinbarung des Verwendungszwecks der vom Stillhalteabkommen umfassten Mittel. Problematisch können diese Abreden aber werden, wenn die am Stillhalteabkommen beteiligten Finanzgläubiger dem Schuldner allzu konkrete Vorgaben machen und sich damit organtypisch verhalten bzw. einzelne, konkrete Geschäftsführungshandlungen vorgeben. So wird es in aller Regel unproblematisch sein, wenn sich der Schuldner in einem Stillhalteabkommen verpflichtet, allgemeine Sanierungsmassnahmen zu treffen oder einen Sanierungsberater beizuziehen. Demgegenüber ist kritisch, wenn die Finanzgläubiger dem Schuldner im Stillhalteabkommen konkret vorgeben, welche Sanierungsmassnahmen zu ergreifen sind $^{82}$ oder welcher konkrete Sanierungsberater zu engagieren ist. ${ }^{83}$ Ebenfalls ist unproblematisch, wenn im Stillhalteabkommen festgehalten wird, dass mit den vom Abkommen erfassten Mitteln keine Zahlungen an Aktionäre (Dividendenzahlungen, Rückzahlungen von Aktionärsdarlehen etc.) getätigt werden dürfen, dass die Mittel nicht für Neuanschaffungen verwendet werden können, oder dass die Mittel nur im Rahmen des ordentlichen Geschäftsbetriebs verwendet werden dürfen. Kritisch werden solche Vorgaben dann, wenn dem Schuldner die konkrete Verwendung der Mittel diktiert wird. ${ }^{84}$ Je konkreter die am Stillhalteabkommen beteiligten Finanzgläubiger dem Schuldner vorgeben, wie er sich zu verhalten hat, desto wahrscheinlicher wird, dass sich die

81 Also der Verzicht auf die Kündigung von Krediten, das Offenhalten von Kreditlinien sowie die Stundung von Forderungen.

82 Beispielsweise wenn dem Schuldner im Stillhalteabkommen vorgegeben wird, auf welchen Märkten er aktiv sein soll, zu welchen Preisen er seine Produkte zu verkaufen hat, mit welchen Zulieferern gearbeitet wird etc.

83 FISCHER R., 40, wonach der blosse Vorschlag eines Sanierungsberaters für den Finanzgläubiger unter dem Gesichtspunkt der faktischen Organschaft unproblematisch ist.

84 Beispielsweise wenn dem Schuldner im Stillhalteabkommen vorgegeben wird, welche Gläubiger zu befriedigen sind (und welche nicht). 
Finanzgläubiger in organtypischer Weise verhalten bzw. Geschäftsführungshandlungen vornehmen bzw. zumindest in erheblichem Umfang beeinflussen und damit als faktische Organe des Schuldners qualifizieren.

Regelmässig als problematisch anzusehen ist die Vereinbarung von Genehmigungsvorbehalten, die Einsitznahme der Finanzgläubiger in Steuerungsausschüssen sowie die Teilnahme der Finanzgläubiger an Sitzungen des Verwaltungsrats ${ }^{85}$ oder der Geschäftsleitung des Schuldners. Auf die Vereinbarung von Genehmigungsvorbehalten sollte in Stillhalteabkommen nach Möglichkeit verzichtet werden. Es besteht ansonsten eine erhebliche Gefahr, dass durch diese Einflussnahme auf den Schuldner die Schranke zur Qualifikation als faktisches Organ überschritten wird. Die Teilnahme an einem Steuerungsausschuss ist je nach dessen Kompetenzen und seiner Funktion problematisch; die Finanzgläubiger sollten nicht die Entscheidträger für den Schuldner sein. Es ist sicherzustellen (und zu protokollieren), dass sich die Finanzgläubiger bei organtypischen Entscheiden in den Ausstand begeben. Wird die Teilnahme an Verwaltungsratssitzungen oder Sitzungen der Geschäftsleitung vereinbart, so ist sicherzustellen, dass sich aus den entsprechenden Protokollen ergibt, dass die Teilnahme der Finanzgläubiger nur zu Konsultationszwecken erfolgte, dass an Abstimmungen nicht teilgenommen wurde, und dass auch sonst keine Beeinflussung der Willensbildung der Organe stattgefunden hat.

\section{b) Tatbestandsmerkmale und Haftung}

\section{aa) Allgemeines}

Entsprechend den allgemeinen haftpflichtrechtlichen Grundsätzen ist Voraussetzung der Haftung stets, dass ein Schaden, also eine finanzielle Einbusse eingetreten ist. Nach der Differenztheorie besteht der Schaden in der Differenz zwischen dem gegenwärtigen Vermögensstand und dem Stand, den das Vermögen ohne das schädigende Ereignis hätte. ${ }^{86}$ Weitere Voraussetzung der aktienrechtlichen Verantwortlichkeit ist die Pflichtverletzung, welche eine besondere Form der Widerrechtlichkeit darstellt. Allgemeine Grundlage ist die Sorgfalts- und Treuepflicht nach Art. 717 Abs. 1 OR sowie die Gleichbehandlungspflicht gemäss Art. 717 Abs. 2 OR. ${ }^{87}$ Haftbar wird nur, wer schuldhaft gehandelt hat, wobei das Verschulden nach einem objektiven Massstab beurteilt wird. Subjektive Entschuldbarkeit ist daher nicht zu beachten. ${ }^{88}$ Schliess-

\footnotetext{
85 Zur Teilnahme eines Kreditgebers an Verwaltungsratssitzungen: BGE 107 II 349.

86 MEIER-HAYOZ/FORSTMOSER/SETHE, § 16, N 806.

87 MEIER-HAYOZ/FORSTMOSER/SETHE, $\$ 16, \mathrm{~N} 808$.

88 MEIER-HAYOZ/ForStMoser/SETHE, § 16, N 820.
} 
lich muss zwischen dem schuldhaft pflichtwidrigen Verhalten und dem Schaden ein Kausalzusammenhang bestehen, wobei die allgemeinen Regeln des Haftpflichtrechts Anwendung finden. ${ }^{89}$

\section{bb) Relevanz für Stillhalteabkommen}

Die allgemeinen Haftungsvoraussetzungen des Verantwortlichkeitsrechts gelten auch für ein faktisches Organ. Für die aktienrechtliche Verantwortlichkeit eines faktischen Organs bedarf es - nebst der Qualifikation als faktisches Organ - mit anderen Worten eines Schadens, einer Pflichtverletzung, eines adäquaten Kausalzusammenhangs zwischen Schaden und Pflichtverletzung sowie eines Verschuldens.

Mit Blick auf das Tatbestandsmerkmal des Schadens bestehen u.E. keine Besonderheiten im Zusammenhang mit Stillhalteabkommen bzw. den am Stillhalteabkommen beteiligten Finanzgläubigern. Erwähnenswert ist immerhin, dass im interessierenden Kontext nur der Schaden relevant sein kann, dessen Ursprung zu einem Zeitpunkt eintrat, als sich der Finanzgläubiger als faktisches Organ des Schuldners qualifizierte.

Ob einem Finanzgläubiger eine verantwortlichkeitsrechtlich relevante Pflichtverletzung vorgeworfen werden kann, kann nur im konkreten Einzelfall beantwortet werden. Praktisch relevant im Kontext von Stillhalteabkommen sind u.E. vor allem zwei potentielle Pflichtverletzungen. Das verspätete Deponieren der Bilanz bzw. die Konkursverschleppung einerseits, und die Ungleichbehandlung von Gläubigern andererseits.

Nach der hier vertretenen Auffassung, kann der am Stillhalteabkommen teilnehmende Finanzgläubiger für eine unterbliebene bzw. verspätete Deponierung der Bilanz in der Regel nicht verantwortlich gemacht werden. Die Pflicht zur Benachrichtigung des Richters gemäss Art. 725 OR obliegt alleine dem (formellen) Verwaltungsrat, der diese Aufgabe nicht delegieren kann.${ }^{90}$ Ein faktisches Organ - bei gegebenen Voraussetzungen also auch der am Stillhalteabkommen teilnehmende Finanzgläubiger - kann deshalb für eine verspätete Benachrichtigung des Richters in aller Regel nicht zur Verantwortung gezogen werden. ${ }^{91}$ Dies ergibt sich direkt aus der Natur der Benachrichtigung des Richters als unentziehbare und unübertragbare Aufgabe des Verwaltungs-

\footnotetext{
89 MEIER-HAYOZ/ForSTMOSER/SETHE, § 16, N 822.

90 Art. 716a Abs. 1 Ziff. 7 OR; Forstmoser/MEIER-HAYOZ/NOBEL, § 50, N 209.

91 Ebenso: AFFENTRANGER, 110.
} 
rats. ${ }^{92}$ Eine Haftung faktischer Organe im Zusammenhang mit einer verspäteten Überschuldungsanzeige kommt nur in Betracht, wenn sie den formellen Verwaltungsrat von der Benachrichtigung abhielten oder es unterliessen, diesen über das Bestehen der Überschuldung zu informieren. ${ }^{93}$ Eine solche Situation dürfte jedoch nur in den wenigsten Ausnahmefällen vorliegen. ${ }^{94}$

Während bei einer aufrechtstehenden Gesellschaft fällige Schulden ohne weiteres bezahlt werden dürfen, verhält es sich dann anders, wenn sich eine Gesellschaft in einer wirtschaftlichen und finanziellen Situation befindet, in welcher zu Liquidationswerten bilanziert werden muss und daraus eine Überschuldung der Gesellschaft resultiert; diesfalls haben die Gläubiger nach Massgabe der gesetzlichen Rangordnung gemäss Art. 219 SchKG Anspruch auf Gleichbehandlung. Jede bevorzugte Befriedigung eines Gläubigers stellt eine Pflichtverletzung dar. ${ }^{95}$ Handelt es sich beim am Stillhalteabkommen teilnehmenden Finanzgläubiger um ein faktisches Organ des Schuldners, und wird im Stillhalteabkommen vereinbart, dass die (ungesicherten) Forderungen der Finanzgläubiger vorab, also bevorzugt zu den andern Gläubigern befriedigt werden, die in der Folge zu Schaden kommen, so kann dies zur Verantwortlichkeit des faktischen Organs führen. Diese Gefahr für den Finanzgläubiger verschärft sich dadurch, dass er sich in einem offenkundigen Interessenkonflikt befindet. Als faktisches Organ hat der Finanzgläubiger die Interessen des Schuldners zu wahren; als Finanzgläubiger geht es ihm allerdings um die Wahrung eigener Interessen. ${ }^{96}$ Wirken die Finanzgläubiger trotz dieses Interessenkonflikts an der Beschlussfassung über Geschäftsentscheide mit, so ist

92 Vgl. auch GlanZmann/Wolf, 37, welche u.E. zu Recht darauf hinweisen, dass das faktische Organ auch keine Pflicht hat, den formellen Verwaltungsrat aktiv zur Deponierung der Bilanz zu bewegen.

93 Urteil des Bundesgerichts 4A_474/2011 vom 4. Januar 2012 E. 3.4.

94 Beispielsweise dann, wenn im Stillhalteabkommen vereinbart wurde, dass eine Benachrichtigung des Richters nur nach vorgängiger Genehmigung durch die Finanzgläubiger zulässig ist, oder wenn ein Steuerungsausschuss, in dem der Finanzgläubiger Einsitz nimmt, über die Deponierung der Bilanz zu entscheiden hat.

95 Urteil des Bundesgerichts 5C.29/2000 vom 19. September 2000 E. 4.b) aa); zur Frage der Aktivlegitimation und der von der Pflichtverletzung betroffenen Vermögensmasse: BGE 142 III 23 E. 4.2. Zur Gleichbehandlungspflicht und Verantwortlichkeit auch: SCHENKER, Oerlikon, $543 \mathrm{f}$.

96 Vgl. WoLF, N 400: „Werden die Banken lediglich als schuldvertragliche Partner zur Wahrung eigener legitimierter Interessen tätig, so kann ihnen dies unter dem Aspekt der aktienrechtlichen Verantwortlichkeit nicht zum Nachteil gereichen. Kommt ihnen hingegen eine faktische Organstellung zu, so wirkt sich häufig gerade die Verfolgung eigener Interessen haftungsbegründend aus." 
die Pflichtverletzung zu vermuten. ${ }^{97}$ Auch ein erfolgreiches Berufen auf die „Business Judgment Rule“ ist unter diesen Vorzeichen nicht möglich. Gemäss der Business Judgment Rule haben Gerichte bei der nachträglichen Beurteilung von Geschäftsentscheiden Zurückhaltung zu üben, wenn sie in einem einwandfreien, auf einer angemessenen Informationsbasis beruhenden und von Interessenkonflikten freien Entscheidungsprozess zustande gekommen sind. ${ }^{98}$ Gerade ein solcher von Interessenkonflikten freier Entscheidungsprozess dürfte mit Blick auf die Finanzgläubiger in diesen Fragen nicht gegeben sein.

Keine Besonderheiten ergeben sich in Bezug auf die Tatbestandselemente des (adäquaten) Kausalzusammenhangs und des Verschuldens. Erwähnenswert ist immerhin, dass sich aus dem Umstand, dass die Pflichtverletzung adäquat kausal für den entstandenen Schaden sein muss ergibt, dass das faktische Organ überhaupt in der Lage gewesen sein muss, den Eintritt des Schadens zu verhindern. ${ }^{99}$

\section{Fazit}

Stillhalteabkommen als private Sanierungsmassnahme bzw. als Bestandteil eines umfassenden Sanierungskonzepts sind in der Praxis weit verbreitet. Im Falle des Scheiterns der Sanierung bergen Stillhalteabkommen indes gewisse Risiken für die teilnehmenden Finanzgläubiger. Diese Risiken können durch eine entsprechende Vertragsgestaltung und das faktische Verhalten der Vertragsparteien minimiert werden.

Kreditrückzahlungen, die im Rahmen von Stillhalteabkommen getätigt werden, können in der Insolvenz des Kreditnehmers unter Umständen paulianisch angefochten werden. Das Risiko wird minimiert bei der Gewährung (und Rückzahlung) von gesicherten Krediten oder wenn Vereinbarungen getroffen werden, die eine Qualifikation als Sanierungsdarlehen erlauben. Die Zahlung von Darlehenszinsen ist unter dem Blickwinkel der paulianischen Anfechtung regelmässig unproblematisch. Die Bezahlung von Darlehenszinsen kann jedoch dann problematisch werden, wenn sie vom Stillhalten mitumfasst und gestundet werden. Die ursprüngliche Besicherung von Krediten ist unproblematisch. Bei der nachträglichen Besicherung von Krediten ist besonderes

\footnotetext{
97 WOLF, N 399.

98 Statt aller: FISCHER D., 279 m.w.H.

99 BGE 136 III 14 E. 2.4 (Pra 2010 Nr. 72); vgl. WOLF, N 813 m.w.H.
} 
Augenmerk auf die finanzielle Situation des Schuldners zu legen. Ganz allgemein sollte im Rahmen von Stillhalteabkommen nach Möglichkeit darauf verzichtet werden, Handlungen gemäss Art. 287 Abs. 1 SchKG zu vereinbaren.

Stillhalteabkommen sind so zu formulieren und zu leben, dass der am Abkommen teilnehmende Finanzgläubiger nicht als faktisches Organ des Kreditschuldners erscheint. Besondere Vorsicht ist angebracht, wenn im Stillhalteabkommen Genehmigungsvorbehalte $\mathrm{zu}$ Gunsten der Finanzgläubiger vereinbart werden, und wenn den Finanzgläubigern das Recht zum Einsitz in ein Steuerungsausschuss oder zur Teilnahme an Sitzungen des Verwaltungsrats und/oder der Geschäftsleitung eingeräumt wird. Insbesondere ist sicherzustellen, dass sich aus den Sitzungsprotokollen ergibt, dass sich die Finanzgläubiger nicht organtypisch verhalten haben, und dass sie nicht die Geschäftsführung für den Schuldner vorgenommen haben.

\section{Literaturverzeichnis}

AfFentranger Markus, Die Stellung der Banken in der Krisensituation - Rechtliche Aspekte, in: Vito Roberto (Hrsg.) Sanierung der AG, Ausgewählte Rechtsfragen für die Unternehmenspraxis, 2. A., Zürich/Basel/ Genf 2003, 103 ff.

AmONn KurT/WALther Fridolin, Grundriss des Schuldbetreibungs- und Konkursrechts, 9. A., Bern 2013.

Basler Kommentar zum SchKG, Bundesgesetz über Schuldbetreibung und Konkurs II (Art. 159-352 SchKG), Staehelin Daniel/Bauer Thomas (Hrsg.), 2. A., Basel 2016 (zit. BSK SchKG-BEARBEITER, Art. XX, N YY).

Bernheim Marc/Geiger Gaudenz, Paulianische Anfechtung - Auswirkungen der Beweislastverteilung aus beratender und forensischer Sicht, in: Sprecher Thomas (Hrsg.), Sanierung und Insolvenz von Unternehmen VI, Zürich/Basel/Genf 2014, 7 ff.

Fischer DAMIAN, Organisation und Haftung im Aktienrecht, AJP 2020, 271 ff. (zit. FISCHER D.).

Fischer Roland, Die kreditgebende Bank im Debt-Work-out Prozess ihres Schuldners Handlungsoptionen und Risiken, in: Sprecher Thomas (Hrsg.), Sanierung und Insolvenz von Unternehmen III, Zürich 2013, 27 ff. (zit. FISCHER R.).

Forstmoser Peter/Meier-Hayoz Arthur/Nobel Peter, Schweizerisches Aktienrecht, Bern 1996.

FORSTMOSER PETER, Die aktienrechtliche Verantwortlichkeit, 2. A., Zürich 1987.

GLANZMANN LUKAS/WOLF MARKUS, Haftung faktischer Organe für Schäden aus Konkursverschleppung, in: Sprecher Thomas (Hrsg.), Sanierung und Insolvenz von Unternehmen IX, Zürich/Basel/Genf 2019, 21 ff.

GLANZMANN LUKAS, Sanierungsdarlehen, ZBJV 146/2010, $261 \mathrm{ff}$.

KRIESI KatHrin, Actio Pauliana, Anfechtbare Handlungen nach Art. 285 ff. SchKG, Zürich 2020. 
Kunz Peter V., Materielle Organschaft („faktische VR“): Voraussetzung sowie Folgen im Aktienrecht, in: Kunz Peter V./Jörg Florian S./Arter Oliver(Hrsg.), Entwicklungen im Gesellschaftsrecht IX, Bern 2014, $174 \mathrm{ff}$.

Kurzkommentar SchKG, Schuldbetreibungs- und Konkursgesetz, in: Hunkeler Daniel (Hrsg.), 2. A., Basel 2014 (zit. KUKO SchKG-BEARBEITER, Art. XX, N YY).

Meier-Hayoz Arthur/Forstmoser Peter/Sethe Rolf, Schweizerisches Gesellschaftsrecht, 12. A., Zürich 2018.

ROTH JÜRG, Die Behandlung von Krisendarlehen im Konkurs, in: Sprecher Thomas (Hrsg.), Sanierung und Insolvenz von Unternehmen, Zürich/Basel/Genf 2011, $41 \mathrm{ff}$.

Rubli ARMAND P., Sanierungsmassnahmen im Konzern aus gesellschaftsrechtlicher Sicht, Zürich/Basel/Genf 2002.

SCHENKER URS, OC Oerlikon, Eine komplexe, aber erfolgreiche Restrukturierung, GesKR 4/ 2010, 542 ff. (zit. SCHENKER, Oerlikon).

SCHENKER URS, Möglichkeiten zur privatrechtlichen Sanierung von Aktiengesellschaften, SJZ 105/2009, 485 ff. (zit. SCHENKER, Sanierung).

SCHENKER URS, Sanierung und Restrukturierung von Unternehmen - Rechtliche und steuerliche Rahmenbedingungen, Zürich, 2015, unter <https://www.walderwyss.com/ user assets/publications/1698.pdf> (zit. SCHENKER, Restrukturierung).

SCHMID CHRISTOPH, Sanierungen aus Bankensicht - aus der Praxis der Recovery Abteilung einer Grossbank, in: Sprecher Thomas (Hrsg.), Sanierung und Insolvenz von Unternehmen III, Zürich 2013, 155 ff.

Schulthess Kommentar, Kommentar zum Bundesgesetz über Schuldbetreibung und Konkurs SchKG, Kostkiewicz Jolanta Kren/Vock Dominik (Hrsg.), 4. A., Bern/Zürich 2017 (zit. SK SchKG-BEARBEITER, Art. XX, N YY).

SPRECHER THOMAS/SOMMER CHRISTA, Aktienrechtliche Sanierungsmassnahmen, in: Sprecher Thomas (Hrsg.), Sanierung und Insolvenz von Unternehmen VI, Zürich/ Basel/ Genf $2014,58 \mathrm{f}$

VANDEBROEK Jos, Ein Weg zum Sanierungsdarlehen im OR, Jusletter, 25. Mai 2020

WOLF MARKUS, Stillhalteabkommen kreditgebender Banken, Ein Beitrag zur Unternehmenssanierung, Zürich/St. Gallen 2012. 


\title{
Die Sanierung im neuen Aktienrecht
}

\author{
Brigitte Knecht/Livia Keller*
}

\section{Inhalt}

I. Einleitung 122

II. Revision des Aktienrechts, inklusive Revision des Sanierungsrechts 123

1. Stand der Revision des Sanierungsrechts 123

2. Hauptziel der Revision des Sanierungsrechts 124

III. Sanierung im geltenden Aktienrecht 125

1. Übersicht 125

2. Begrifflichkeiten: „Kapitalverlust“ / „Überschuldung“ 126

3. Handlungspflichten des Verwaltungsrats bei Kapitalverlust 126

4. Handlungspflichten des Verwaltungsrats bei Überschuldung 127

a) Bei begründeter Besorgnis einer Überschuldung 127

b) Bei Überschuldung 128

5. Exkurs: Spezialregelungen aufgrund von COVID-19 130

a) Neuerungen aufgrund der COVID-19-Verordnung Insolvenzrecht $\quad 130$

b) Neuerungen aufgrund der COVID-19-Solidarbürgschaftsverordnung 131

IV. Sanierung im neuen Aktienrecht: Was ändert sich? 131

1. Übersicht über die Tatbestände 131

2. Drohende Zahlungsunfähigkeit 132

a) Übersicht 132

b) Begriff „Zahlungsunfähigkeit“ 133

c) Handlungspflichten des Verwaltungsrats 134

aa) Im Allgemeinen 134

bb) Bei drohender Zahlungsunfähigkeit 135

d) Was ändert sich? 136

3. Kapitalverlust 137

a) Übersicht 137

b) Begriff „Kapitalverlust“ 138

c) Handlungspflichten des Verwaltungsrats 138

d) Was ändert sich? 139

4. Bei Überschuldung 140

a) Übersicht 140

* Der vorliegende Text ist die schriftliche Fassung des Referats, das die Autorinnen anlässlich der 11. Tagung zur Sanierung und Insolvenz von Unternehmen vom 9. Juni 2020 gehalten haben. Der Stand der verwendeten Literatur und Materialien sowie der Gesetzgebung entspricht ebenfalls diesem Datum. 
b) Begriff „Überschuldung“ 141

c) Handlungspflichten des Verwaltungsrats 141

a) Bei begründeter Besorgnis einer Überschuldung 141

bb) Bei Überschuldung 142

cc) Vorgaben in zeitlicher Hinsicht 144

dd) Rangrücktritt 145

ee) Stille Sanierung 146

d) Was ändert sich? 147

V. Sanierungsmassnahmen 148

1. Bilanzierungsmassnahmen 149

2. Änderung der Kapitalstruktur / Liquiditätsverbesserung 149

3. Operationelle Massnahmen 149

4. Aufwertung von Grundstücken und Beteiligungen im Besonderen 150

VI. Würdigung 151

Literaturverzeichnis 152

\section{Einleitung}

Am 19. Juni 2020 verabschiedete das Parlament nach jahrzehntelangen Vorarbeiten die Aktienrechtsrevision und damit die Revision des Sanierungsrechts. ${ }^{1}$ Der vorliegende Beitrag wird die vom Parlament verabschiedeten Änderungen des aktienrechtlichen Sanierungsrechts näher beleuchten und dabei zeigen, inwieweit sich zum geltenden Recht etwas ändern wird.

Damit dieser Vergleich gelingen kann, wird nach einer kurzen Einführung zum Stand und den Hauptzielen der Revision des aktienrechtlichen Sanierungsrechts (vgl. unten, II.) zunächst ein Überblick über das geltende Recht gegeben (vgl. unten, III.). Dabei wird in der gebotenen Kürze auch auf die aktuellste Entwicklung im Zusammenhang mit dem COVID-19-Notrecht des Bundesrats hingewiesen, soweit dieses Notrecht zu einer gewissen - vorübergehenden - Modifikation von Art. 725 des Bundesgesetzes betreffend die Ergänzung des Schweizerischen Zivilgesetzbuches (Fünfter Teil: Obligationenrecht) vom 30. März 1911 (OR, SR 220) geführt hat.

Den Kern der sanierungsrechtlichen Neuerungen im Aktienrecht bilden die vom Parlament mit Beschluss vom 19. Juni 2020 verabschiedeten nArt. 725 bis 725c des Obligationenrechts (OR, BBl 2020, 5573 ff.). Diesen privatrechtlichen Sanierungsbestimmungen widmet sich der vorliegende Beitrag schwer-

1 Beschluss der Bundesversammlung der Schweizerischen Eidgenossenschaft vom 19. Juni 2020 über die Änderung des Obligationenrechts, BBl 2020, 5573 ff. 
gewichtig (vgl. unten, IV. und V.). Die öffentlich-rechtliche Sanierung unter Mitwirkung des Gerichts nach den Bestimmungen des Bundesgesetzes über Schuldbetreibung und Konkurs vom 11. April 1889 (SchKG, SR 281.1) bildet hingegen nicht Gegenstand der vorliegenden Publikation.

Abschliessend wird im Rahmen einer Würdigung der Frage nachgegangen, ob und inwieweit die Hauptziele der Revision des Sanierungsrechts mit den nunmehr verabschiedeten neuen Bestimmungen, die wesentlich vom Entwurf des Bundesrats abweichen, erreicht werden können (vgl. unten, VI.).

\section{Revision des Aktienrechts, inklusive Revision des Sanierungsrechts}

\section{Stand der Revision des Sanierungsrechts}

Die Aktienrechtsreform und damit die Revision des Sanierungsrechts hat bereits einen langen Weg in den politischen Gremien hinter sich. Die sanierungsrechtlichen Bestimmungen des Aktienrechts bilden lediglich einen kleinen Bestandteil dieses umfassenden Reformpakets. ${ }^{2}$

Als Auslöser der Revision des aktienrechtlichen Sanierungsrechts kann das Swissair-Grounding im Herbst 2001 angesehen werden. Der Zusammenbruch der Swissair, bei welchem unter anderem akute Liquiditätsprobleme eine entscheidende Rolle spielten ${ }^{3}$, bewog den Gesetzgeber zunächst zu einer Teilrevision des SchKG. ${ }^{4}$ Das revidierte Sanierungsrecht des SchKG ist bekanntlich am 1. Januar 2014 in Kraft getreten. ${ }^{5}$ Im Rahmen der parlamentarischen Beratung dieser Vorlage wurde der Bundesrat im Jahr 2012 mit zwei gleichlautenden Motionen der national- und ständerätlichen Rechtskommissionen beauftragt,

2 Für einen Überblick über alle Neuerungen vgl. z.B. FORSTMOSER/KÜCHLER, $3 \mathrm{ff}$.

3 Glanzmann, Sanierung, 470 Fn. 45 m.V.a. Bericht der Geschäftsprüfungskommission des Ständerates vom 19. September 2002 über die Rolle von Bundesrat und Bundesverwaltung im Zusammenhang mit der Swissair-Krise, BBl 2003, 5403 ff., 5482 f.: „Die Rechnungen der Lieferanten mussten ab sofort bar beglichen werden. Die Swissair verfügte jedoch nicht über genügend Geld, um dies zu tun, umso mehr als auch die noch ausstehenden Rechnungen bar bezahlt werden mussten.“; vgl. dazu auch SF Dokumentarfilm „Als die Schweiz den Atem anhielt: Das Drama der Swissair“, 2011 (abrufbar unter <https://www.srf.ch/ play/tv/katastrophen/video/als-die-schweiz-den-atem-anhielt-das-drama-der-swissair?id=85f6d89e-5e26-4735-9e5e-927a027cdae3 $>$ ).

4 SPRECHER/SOMMER, Sanierungsmassnahmen, 37.

5 Für eine Übersicht über die Änderungen im SchKG vgl. Beschluss der Bundesversammlung der Schweizerischen Eidgenossenschaft vom 21. Juni 2013, AS 2013, 4111 ff. 
ein umfassendes Sanierungsverfahren im Obligationenrecht vorzulegen. ${ }^{6}$ Dieses soll - als Gegenstück zum Sanierungsrecht des SchKG - die Sanierung von Unternehmen vor der Einleitung eines formellen öffentlichen Nachlassverfahrens ermöglichen bzw. erleichtern.

Am 19. Juni 2020 verabschiedete das Parlament die Aktienrechtsreform und damit die Revision des Sanierungsrechts. Momentan läuft die Referendumsfrist, die am 8. Oktober 2020 ablaufen wird. ${ }^{8}$ Was das Inkrafttreten der neuen Bestimmungen angeht, ist es naturgemäss schwierig, eine Prognose abzugeben. Unserer Einschätzung nach ist aber kaum mit einem Inkrafttreten vor dem Jahr 2022 zu rechnen. ${ }^{9}$

\section{Hauptziel der Revision des Sanierungsrechts}

Ziel der aktienrechtlichen Sanierung war bereits bisher und ist nach wie vor, die finanzielle Schieflage eines Unternehmens unter dessen optimaler Werterhaltung zu beseitigen. ${ }^{10}$ Unter geltendem Recht ist man aber oft damit konfrontiert, dass die Sanierungsmassnahmen zu spät einsetzen, um eine erfolgreiche aussergerichtliche Sanierung ermöglichen zu können. ${ }^{11}$

Das geltende Obligationenrecht legt bei Kapitalgesellschaften ein starkes Augenmerk auf die Aufbringung und Erhaltung des Eigenkapitals. Mit dem Verlust des freien Eigenkapitals büsst eine Gesellschaft ihre Dividendenfähigkeit ein. Entsprechend muss der Verwaltungsrat nach der geltenden gesetzlichen Konzeption sowohl bei Eintritt eines Kapitalverlusts als auch bei begründeter Besorgnis bzw. bei Vorliegen einer Überschuldung bestimmte

6 Motionen der national- und ständerätlichen Kommissionen für Rechtsfragen vom 21. Mai und 28. Juni 2012 betreffend „Sanierungsverfahren vor Nachlassstundung und Konkurseröffnung“ (12.3403 und 12.3654).

7 Botschaft des Bundesrates vom 23. November 2016 zur Änderung des Obligationenrechts (Aktienrecht), BBl 2017, 399 ff., 462; ForSTMOSER/KÜCHLER, 7; SPRECHER/SOMMER, Sanierung, 551 .

8 BBl 2020, $5573 \mathrm{ff}$.

9 Vgl. auch ForSTMOSER/KÜCHLER, 26 f., die ein Inkrafttreten erst im Verlauf des Jahres 2021 oder dem 1. Januar 2022 als sinnvoll betrachten, um den Gesellschaften, Anwälten, Unternehmensjuristen und Treuhändern ausreichend Zeit zu geben, sich mit dem neuen Recht vertraut zu machen und die nötigen Anpassungen vorzunehmen.

10 SPRECHER/SOMmER, Sanierung, 551; SCHENKER, 487.

11 GlanZMAnN, Sanierung, 470; BöCKLI, Aktienrecht, § 13 N 817k; vgl. ferner BAUER, 456 f. 
Massnahmen ergreifen. ${ }^{12}$ Als Grundsatz gilt: „Je tiefer das Eigenkapital, desto enger wird der Handlungsspielraum der Gesellschaft und desto mehr Pflichten werden der Gesellschaft und ihren Organen auferlegt ${ }^{\text {“13 }}$.

In der Unternehmenspraxis ist aber nicht primär das Eigenkapital, sondern die Zahlungsfähigkeit für den Bestand einer Gesellschaft zentral ${ }^{14}$ - dies konnte man etwa beim Grounding der Swissair sehen. ${ }^{15}$ Deshalb ist es erstaunlich, dass das geltende aktienrechtliche Sanierungsrecht die Liquidität nicht explizit erwähnt. ${ }^{16}$

Den Motionen der national- und ständerätlichen Rechtskommissionen folgend sollte das Hauptziel der Revision des Sanierungsrechts des Obligationenrechts darin liegen, „präzisere und z.T. neue Handlungspflichten für die Unternehmen bzw. deren Verwaltungsrat einzuführen, damit möglichst frühzeitig Sanierungsschritte erfolgen ${ }^{\text {“17 }}$. Zur Erreichung dieses Ziels wurde in erster Linie eine Neugestaltung der „Frühwarnsysteme ${ }^{\text {“18 }}$ ins Auge gefasst: „Das Bewusstsein des Verwaltungsrates bezüglich Liquidität und Kapitaldeckung sollte geschärft werden, indem sowohl seine Handlungsspielräume erweitert und seine Pflichten konkretisiert werden ${ }^{\text {“19. }}$.

Wie bereits erwähnt, widmet sich der vorliegende Beitrag unter anderem der Frage, ob und inwieweit diese Hauptziele der Revision des Sanierungsrechts mit den nunmehr verabschiedeten neuen Bestimmungen erreicht werden können. Dazu werden die neuen Bestimmungen nach einem Überblick über das geltende Recht vorgestellt und mit Letzterem verglichen.

\section{Sanierung im geltenden Aktienrecht}

\section{I. Übersicht}

Die sanierungsrechtlichen Bestimmungen im geltenden Aktienrecht finden sich in Art. 725 f. OR. Durch diese Bestimmungen wird der Verwaltungsrat für

\footnotetext{
12 Glanzmann, Sanierung, 465 f.; Glanzmann, Dividendenausschüttungen, 100 f.; GlanZmann, Pflichten, $20 \mathrm{f}$.

13 Glanzmann, Sanierung, $465 \mathrm{f}$.

14 SPREChER/SOMmer, Sanierung, 555; BÜHLER, Sanierung, 19 f.; BSK OR II-WÜSTINER, Art. 725, N 9a; Glanzmann, Sanierung, 465.

15 Vgl. dazu oben, Fn. 3.

16 Glanzmann, Sanierung, 466.

17 BBl 2017, 462.

18 BBl 2017, $462 \mathrm{f}$.

19 BBl 2017, 463.
} 
den Fall, dass das Eigenkapital unter eine bestimmte Marke sinkt, anhand eines kaskadenartigen Systems ausdrücklich verpflichtet, bestimmte Massnahmen zu ergreifen: Das Gesetz knüpft dabei an den sog. Kapitalverlust nach Art. 725 Abs. 1 OR und die sog. Überschuldung nach Art. 725 Abs. 2 OR an.

\section{Begrifflichkeiten: „Kapitalverlust“ / „Überschuldung“}

Für die Beurteilung, ob ein Kapitalverlust oder eine Überschuldung vorliegt, ist einzig der Einzelabschluss nach Obligationenrecht massgebend. ${ }^{20}$

Ein Kapitalverlust im Sinne von Art. 725 Abs. 1 OR ist gegeben, wenn die Aktiven neben dem Fremdkapital nicht mehr die Hälfte des Aktienkapitals (inkl. Partizipationskapital ${ }^{21}$ ) und der gesetzlichen Reserven decken. Es handelt sich um eine qualifizierte Form eines Bilanzverlusts. ${ }^{22}$

Von einer Überschuldung im Sinne von Art. 725 Abs. 2 OR spricht man, wenn die Forderungen der Gesellschaftsgläubiger weder zu Fortführungswerten (also unter der Annahme der Weiterführung der Geschäftstätigkeit ${ }^{23}$ ) noch zu Veräusserungswerten (also unter der Annahme der Liquidation der Gesellschaft ${ }^{24}$ ) gedeckt sind. Im Gegensatz zum Kapitalverlust ist bei der Überschuldung das Eigenkapital der Gesellschaft vollständig aufgezehrt, mithin ist das Fremdkapital nicht mehr voll durch die Aktiven gedeckt. ${ }^{25}$

\section{Handlungspflichten des Verwaltungsrats bei Kapitalverlust}

Liegt ein Kapitalverlust im Sinne von Art. 725 Abs. 1 OR vor, muss der Verwaltungsrat unverzüglich eine Generalversammlung einberufen und ihr Sanierungsmassnahmen beantragen. Entgegen dem Wortlaut der Norm ist die primäre Pflicht des Verwaltungsrats die Erarbeitung von Sanierungsmassnahmen

GlanZmann, Sanierung, 467 und 470; Druey/Druey Just/Glanzmann, § 8 N 57; Böckli, Aktienrecht, § $13 \mathrm{~N} 770 \mathrm{~b}$.

21 Art. 656a Abs. 2 OR; BSK OR II-WÜSTINER, Art. 725, N 20; BÖCKLI, Aktienrecht, § 13 N 717.

22 Vgl. BSK OR II-WÜSTINER, Art. 725, N 33 f. und 37 f.; BSK OR II-NEUHAUS/SUTER, Art. 958a, N 1.

23 MEIER-HAYOZ/ForSTMOSER/SETHE, § 16 N 125.

24 MEIER-HAYOZ/FORSTMOSER/SETHE, § $16 \mathrm{~N} 125$.

25 ForSTMOSER/MEIER-HAYOZ/NOBEL, § 50 N 205 und ferner N 193; BSK OR II-WÜSTINER, Art. 725, N 29. 
und nicht die blosse Einberufung einer Generalversammlung. ${ }^{26}$ Bei dieser Handlungspflicht handelt es sich um eine unübertragbare und unentziehbare Aufgabe des Verwaltungsrats. ${ }^{27}$

Die sog. Sanierungsversammlung dient einerseits der Information der Aktionäre über die Sanierungsbedürftigkeit der Gesellschaft sowie die beabsichtigten Sanierungsmassnahmen. Andererseits soll dadurch der Generalversammlung - soweit dies in ihren Kompetenzbereich fällt - die Möglichkeit eröffnet werden, über Sanierungsmassnahmen zu beschliessen. ${ }^{28}$

In der Praxis und der Rechtsprechung spielt der Kapitalverlust eine eher untergeordnete Rolle; die sehr viel wichtigere Bestimmung ist Art. 725 Abs. 2 OR, also die Überschuldung. ${ }^{29}$

\section{Handlungspflichten des Verwaltungsrats bei Überschuldung \\ a) Bei begründeter Besorgnis einer Überschuldung}

Nach dem Wortlaut von Art. 725 Abs. 2 OR muss der Verwaltungsrat bei begründeter Besorgnis einer Überschuldung eine Zwischenbilanz zu Fortführungs- und Veräusserungswerten erstellen. Dabei ist gemäss Praxis und Rechtsprechung in einem ersten Schritt ein Abschluss zu Fortführungswerten (also unter der Annahme der Weiterführung der Geschäftstätigkeit ${ }^{30}$ ) zu machen. ${ }^{31}$ Gemeinhin wird davon ausgegangen, dass eine Umstellung auf Veräusserungswerte (also unter der Annahme der Liquidation der Gesellschaft ${ }^{32}$ ) erforderlich ist, wenn klare Anzeichen erkennbar sind, dass die Liquidität für die Fortführung der Geschäftstätigkeit voraussichtlich nicht ausreicht. Dies ist aber noch nicht bei jedem Liquiditätsengpass der Fall, sondern erst, wenn die Gesellschaft von einer eigentlichen Zahlungsunfähigkeit bedroht ist. Der

26 GlanZmann, Sanierung, 468; KrnetA, 2186; BSK OR II-WÜSTINER, Art. 725, N 24; ZK OR-HOMBURGER, Art 725, N 1225.

27 GlanZmann, Sanierung, 468; GlanZmann, Haftungsrisiken, 274; SCHENKER, 485 f.; HANDSCHIN, 439.

28 Glanzmann, Sanierung, 468; Glanzmann, Pflichten, 51; Druey/Druey Just/GlanzmanN, $§ 8$ N 58; HANDSCHIN, 443.

29 GlanZMANN, Sanierung, 470.

30 MEIER-Hayoz/ForSTMOSER/SETHE, § 16 N 125.

31 GlanZmanN, Sanierung, 471; Urteil des Bundesgerichts 4A_373/2015 vom 26. Januar 2016 E. 3.1.2.

32 MEIER-HAYOZ/FORSTMOSER/SETHE, $§ 16$ N 125. 
Abschluss zu Veräusserungswerten ist somit nur dann erforderlich, wenn die Fortführungsprognose nicht mehr positiv ist oder die Zwischenbilanz zu Fortführungswerten eine Überschuldung aufweist. ${ }^{33}$

Gemäss Art. 725 Abs. 2 OR müssen die Zwischenbilanzen von einem zugelassenen Revisor geprüft werden. Obwohl nach einem Teil der Lehre diese Aufgabe nicht zwingend der Revisionsstelle obliegt, wird diese Prüfung in den allermeisten Fällen durch die Revisionsstelle vorgenommen. ${ }^{34}$ Im Falle eines Opting-out muss der Verwaltungsrat einen zugelassenen Revisor zur Prüfung der Zwischenbilanzen bezeichnen. ${ }^{35}$

\section{b) Bei Überschuldung}

Ist die Gesellschaft im Sinne des Gesetzes überschuldet, hat der Verwaltungsrat gemäss Art. 725 Abs. 2 OR grundsätzlich den Richter zu benachrichtigen und diesem die Eröffnung des Konkurses ${ }^{36}$, die Gewährung eines Konkursaufschubs $^{37}$ oder die Gewährung einer Nachlassstundung ${ }^{38}$ zu beantragen. Unterlässt der Verwaltungsrat die Benachrichtigung des Gerichts, wird die Revisionsstelle gemäss Art. 725 Abs. 3 OR diesbezüglich in die Pflicht genommen und zwar insoweit, als sie bei offensichtlicher Überschuldung der Gesellschaft selber das Gericht benachrichtigen muss. ${ }^{39}$

Auf die Benachrichtigung des Gerichts kann verzichtet werden, wenn Rangrücktritte von Gesellschaftsgläubigern vorliegen, welche die Überschuldung abdecken. ${ }^{40}$ Sofern seit dem Bilanzstichtag weitere Verluste angefallen sind, müssen auch diese vom Rangrücktritt abgedeckt werden. ${ }^{41}$ Die Revisionsstellen verlangen zudem oft, dass die bis zum nächsten Bilanzstichtag zu

\footnotetext{
33 BSK OR II-WÜSTINER, Art. 725, N 19.

34 BÖCKLI, Aktienrecht, § 13 N 773; a.M. BSK OR II-WÜSTINER, Art. 725, N 39c; vgl. zum Ganzen auch GLANZMANN, Sanierung, 471.

35 Druey/Druey Just/GLANZMANN, § 8 N 64a; BSK OR II-WÜSTINER, Art. 725, N 39c.

36 Art. 725a Abs. 1 Satz 1 OR.

37 Art. 725a Abs. 1 Satz 2 OR.

38 Art. 293 lit. a SchKG.

39 Art. 728c Abs. 3 und Art. 729c OR.

40 Art. 725 Abs. 2 Satz 2 OR.

41 Glanzmann, Sanierung, 472.
} 
erwartenden Verluste ebenfalls abgedeckt werden. ${ }^{42}$ Nach Rechtsprechung und Praxis ist aber nicht notwendig, dass Zinsen und andere Kosten gestundet werden. ${ }^{43}$

Beim Rangrücktritt handelt es sich um eine nachträgliche Erklärung eines Gläubigers, Befriedigung seiner Forderung für den Fall des Konkurses oder der Liquidation erst und nur dann zu verlangen, wenn sämtliche übrigen Gläubiger voll befriedigt worden sind; ${ }^{44}$ er beinhaltet somit einen Vertrag zu Gunsten Dritter gegenüber allen übrigen Gläubigern, welcher weder der Zustimmung der Aktionäre noch der übrigen Gläubiger bedarf. ${ }^{45}$ Der Rangrücktritt kann eine Sanierung begünstigen, da er einen Kapitalabfluss verhindert; er hat aber keine direkte Eigenkapital- oder Liquiditätsvergrösserung zur Folge. ${ }^{46}$

Neben dem Vorliegen eines Rangrücktritts, welcher explizit im Gesetz vorgesehen ist, kann von der Benachrichtigung des Gerichts nach der Rechtsprechung des Bundesgerichts ferner abgesehen werden, wenn der Verwaltungsrat die Gesellschaft unverzüglich im Sinne einer sog. stillen Sanierung saniert, also ,in einer schwierigen Lage tut, was vernünftigerweise von einem Unternehmer erwartet werden darf ${ }^{447}$. $^{48}$ Ein Verzicht auf die Benachrichtigung des Gerichts ist nach Ansicht des Bundesgerichts indes nur pflichtgemäss, wenn „Aussichten für eine kurzfristig realisierbare Sanierung“49 bestehen. Erforderlich ist, dass die Forderungen der Gesellschaftsgläubiger nicht durch eine neuerliche Verschlechterung der finanziellen Lage gefährdet werden und eine dauerhafte finanzielle Gesundung der Gesellschaft sowie die Wiederherstellung der Ertragskraft erwartet werden können. ${ }^{50}$ Bei vernünftiger Aussicht auf

42 GlanZmanN, Sanierung, 472; Schweizer Handbuch, 257.

43 Urteil des Bundesgerichts 4C.47/2003 vom 2. Juli 2003 E. 4; BSK OR II-WÜSTINER, Art. 725, N 46; BöCKLI, Aktienrecht, § 13 N 794 und 808; FORSTMOSER/MEIER-HAYOz/NOBEL, § 50 N 236 ff. und 673.

44 Meier-Hayoz/Forstmoser/Sethe, § 16 N 125; BSK OR II-WÜStiner, Art. 725, N 45 f.

45 Vgl. zum Ganzen BÖCKLI, Aktienrecht, § 13 N 798; BSK OR II-WÜSTINER, Art. 725, N 45 f.

46 BSK OR II-WÜSTINER, Art. 725, N 47; BÖCKLI, Aktienrecht, § 13 N 793 und 805.

47 BGE 116533 E. 5a S. 541 m.V.a. ForSTMOSER, Verantwortung, 843.

48 BGE 116533 E. 5a S. 541; ForSTMOSER, Verantwortung, 843; vgl. zum Ganzen auch GLANZMANN, Sanierung, 472.

49 BGE 127 IV 110 E. 5a S. 113; Urteil des Bundesgerichts 4C.436/2006 vom 18. April 2007 E. 4.1; vgl. zum Ganzen auch GLanZMAnN, Sanierung, 472.

50 GlanZmanN, Sanierung, 472; Urteil des Bundesgerichts 4C.366/2000 vom 19. Juni 2001 E. 4b; Urteil des Bundesgerichts 6B.492/2009 vom 18. Januar 2010 E. 2.2; BGE 132 III 564 E. 5.1 S. 573 . 
eine Sanierung halten es das Bundesgericht ${ }^{51}$ und die wohl h.L. ${ }^{52}$ für zulässig, dass der Verwaltungsrat die Benachrichtigung des Gerichts gemäss Art. 725 Abs. 2 OR für eine verhältnismässig kurze Zeitspanne von vier bis sechs Wochen aufschiebt und Sanierungsmassnahmen versucht. ${ }^{53}$ Ob diese Frist bei guten Sanierungsaussichten allenfalls auch länger aufgeschoben werden kann, ist umstritten. ${ }^{54}$

\section{Exkurs: Spezialregelungen aufgrund von COVID-I9}

Aufgrund der Aktualität des Themas wollen wir ganz kurz auf die Entwicklung im Zusammenhang mit dem COVID-19-Notrecht des Bundesrats hinweisen, soweit dieses Notrecht zu einer gewissen Modifikation von Art. 725 OR geführt hat. Da es sich dabei jedoch lediglich um vorübergehende Anpassungen handelt, wird für die Zwecke des vorliegenden Beitrags, der sich schwergewichtig mit den Sanierungsbestimmungen im neuen Aktienrecht beschäftigt, auf eine Beschreibung der notrechtlichen Bestimmungen verzichtet und für die Details auf die entsprechenden Verordnungen des Bundesrats verwiesen.

\section{a) Neuerungen aufgrund der COVID-19-Verordnung Insolvenzrecht}

Am 20. April 2020 ist die bundesrätliche Verordnung über insolvenzrechtliche Massnahmen zur Bewältigung der Coronakrise vom 16. April 2020 (COVID-19-Verordnung Insolvenzrecht, SR 281.242) in Kraft getreten. ${ }^{55}$ Sie gilt grundsätzlich für die Dauer von sechs Monaten ab Inkrafttreten, d.h. bis 20. Oktober $2020 .^{56}$

Die COVID-19-Verordnung Insolvenzrecht führt diverse vorübergehende Neuerungen in das bestehende Schweizer Insolvenzrecht ein und enthält insbesondere Anpassungen der Anzeigepflicht im Falle einer Überschuldung gemäss Art. 725 Abs. 2 OR. ${ }^{57}$

51 BGE 76 I 162 E. 3 S. 167; BGE 108 V 183 E. 2 S. 188; BGE 116 II E. 5a S. 541; BGE 132 III 564 E. 5.1 S. 573; Urteil des Bundesgerichts 4C.436/2006 vom 18. April 2007 E. 4 ff.

52 Vgl. statt vieler: BÖCKLI, Aktienrecht, § $13 \mathrm{~N} 816$.

53 SPRECHER/SOMMER, Sanierung, 555; vgl. dazu auch die umfassende Zusammenstellung von Rechtsprechung und Doktrin in FORSTMOSER, Krisenmanager, 369 ff. und 372 ff.; vgl. ferner MEIER-HAYOZ/FORSTMOSER/SETHE, § 16 N 131.

54 Bejahend ForSTMOSER, Krisenmanager, 280 f.; verneinend BöckLI, Aktienrecht, §13 N 777 und 817h ff., vgl. zum Ganzen SPRECHER/SOMMER, Sanierung, 555.

55 Art. 23 Abs. 1 COVID-19-Verordnung Insolvenzrecht.

56 Art. 23 Abs. 2 COVID-19-Verordnung Insolvenzrecht.

57 COVID-19-Verordnung Insolvenzrecht; vgl. auch Erläuterungen des EJPD vom 18. Mai 2020 zu den einzelnen Bestimmungen der COVID-19-Verordnung Insolvenzrecht, $1 \mathrm{ff}$. 


\section{b) Neuerungen aufgrund der COVID-19-Solidarbürgschaftsverordnung}

Art. 725 Abs. 1 und 2 OR werden ferner durch Art. 24 der Verordnung zur Gewährung von Krediten und Solidarbürgschaften in Folge des Coronavirus vom 25. März 2020 (COVID-19-Solidarbürgschaftsverordnung, SR 951.261) modifiziert. Gemäss Art. 24 der COVID-19-Solidarbürgschaftsverordnung werden für die Berechnung der Deckung von Kapital und Reserven nach Art. 725 Abs. 1 OR und für die Berechnung einer Überschuldung nach Art. 725 Abs. 2 OR Kredite, welche gestützt auf Art. 3 der COVID-19-Solidarbürgschaftsverordnung verbürgt werden, bis zum 31. März 2022 nicht als Fremdkapital berücksichtigt. Mit anderen Worten zählen also die COVID-19 Kredite bis CHF 500'000 bei den Tests, ob ein Kapitalverlust oder eine Überschuldung vorliegt, nicht als Fremdkapital.

\section{Sanierung im neuen Aktienrecht: Was ändert sich?}

\section{I. Übersicht über die Tatbestände}

Mit der mittlerweile vom Parlament beschlossenen Aktienrechtsrevision wird, wie erwähnt, auch das aktienrechtliche Sanierungsrecht revidiert. Dieses sollte namentlich besser auf das Sanierungsrecht des SchKG abgestimmt werden. $^{58}$

Das revidierte Sanierungsrecht enthält einzelne Neuerungen im Bereich der Kapitalschutzvorschriften. Als deklariertes Ziel des aktienrechtlichen Sanierungsrechts sollte insbesondere das Bewusstsein des Verwaltungsrats für die zentrale Bedeutung der Liquidität eines Unternehmens geschärft werden. ${ }^{59}$ Entsprechend wird mit nArt. 725 OR ein neuer Tatbestand der drohenden Zahlungsunfähigkeit geschaffen und den bestehenden Tatbeständen des Kapitalverlusts (nArt. 725a OR) und der Überschuldung (nArt. 725b OR), welche modifiziert werden, vorangestellt. Schliesslich wird die Bestimmung von Art. 670 OR betreffend Aufwertung von Grundstücken und Beteiligungen neu in das Sanierungsrecht, konkret in nArt. 725c OR, eingeordnet. ${ }^{60}$

\footnotetext{
58 Forstmoser/Küchler, 7; BBl 2017, 462 ff.; Bundesgesetz über Schuldbetreibung und Konkurs (SchKG), Änderung vom 21. Juni 2013, AS 2013, $4111 \mathrm{ff}$.

$59 \quad \mathrm{BBl} 2017,463$.

60 Vgl. dazu auch GLANZMANN, Sanierung, 476 f.; BBl 2017, 580.
} 

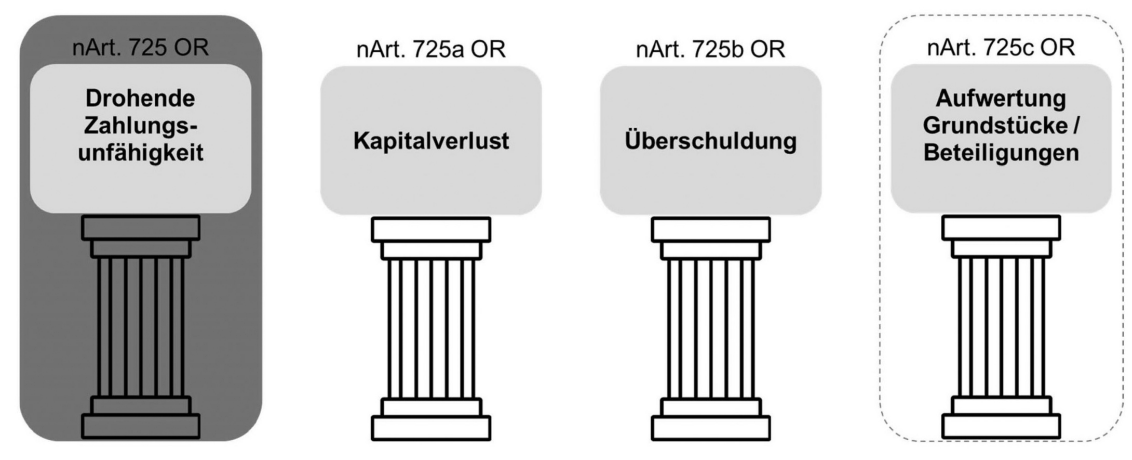

\section{Drohende Zahlungsunfähigkeit}

\section{a) Übersicht}

Nach der geltenden gesetzlichen Konzeption ist der Verwaltungsrat erst und nur bei einem hälftigen Kapitalverlust ausdrücklich zur Ergreifung von Sanierungsmassnahmen verpflichtet. ${ }^{61}$ Zur Stärkung des Kapitalschutzes sollten Sanierungsschritte im Idealfall aber bereits viel früher veranlasst werden. ${ }^{62}$ Denn ist der hälftige Kapitalverlust einmal erreicht, ist der Eigenkapitalschwund oft kaum mehr aufzuhalten. ${ }^{63}$ Beim neuen Tatbestand der drohenden Zahlungsunfähigkeit gemäss nArt. 725 OR sollte daher die Erhaltung der jederzeitigen Fortführungsfähigkeit der Gesellschaft in den Vordergrund gerückt werden. ${ }^{64}$ Neu werden nicht nur bei einer Unterdeckung des Kapitals explizit Handlungspflichten des Verwaltungsrats ausgelöst, sondern bereits bei drohender Zahlungsunfähigkeit. ${ }^{65}$ Dies ist grundsätzlich sinnvoll, denn erfahrungsgemäss sind Unternehmenszusammenbrüche primär auf die mangelnde Liquidität und nicht auf eine (vorübergehende) Überschuldung zurückzuführen. ${ }^{66}$

\footnotetext{
61 Art. 725 Abs. 1 OR.

62 FORSTMOSER/KÜCHLER, 7; BBl 2017, 462 ff.

63 Sprecher/SOMmer, Sanierung, 555; BÖckli, Aktienrecht, § 13 N 749d; BüHLER, Sanierung, 23 f.; von der Crone/Pfister/Sibbern, 9 f. und 12 f.; vOn Der Crone/Kopta-Stutz/Pfister, 1029.

64 HACHMANN, 104.

65 HACHMANN, 119.

66 Sprecher/SOMmer, Sanierung, 555; BÜHLER, Sanierung, 19 f.; MÜLlER, 194; BSK OR II-WÜSTINER, Art. 725, N 91.
} 
Der neue Tatbestand der drohenden Zahlungsunfähigkeit gemäss nArt. 725 OR wird wie folgt lauten:

${ }^{1}$ Der Verwaltungsrat überwacht die Zahlungsfähigkeit der Gesellschaft.

${ }^{2}$ Droht die Gesellschaft zahlungsunfähig zu werden, so ergreift der Verwaltungsrat Massnahmen zur Sicherstellung der Zahlungsfähigkeit. Er trifft, soweit erforderlich, weitere Massnahmen zur Sanierung der Gesellschaft oder beantragt der Generalversammlung solche, soweit sie in deren Zuständigkeit fallen. Er reicht nötigenfalls ein Gesuch um Nachlassstundung ein.

${ }^{3}$ Der Verwaltungsrat handelt mit der gebotenen Eile.

\section{b) Begriff „Zahlungsunfähigkeit“}

Der neue nArt. 725 OR spricht von der drohenden Zahlungsunfähigkeit, enthält aber keine Legaldefinition dieses Begriffs. Gemäss Botschaft liegt Zahlungsunfähigkeit vor, „wenn die Gesellschaft ihre fälligen Verbindlichkeiten nicht mehr erfüllen kann und somit weder über die Mittel verfügt, fällige Verbindlichkeiten zu erfüllen, noch über den erforderlichen Kredit, sich diese Mittel nötigenfalls zu beschaffen ${ }^{467}$.

Beim Test, ob eine Zahlungsunfähigkeit der Gesellschaft droht, sind somit ihre liquiden Mittel, aber auch ihre Kreditwürdigkeit zu berücksichtigen. Die Botschaft präzisiert nicht näher, welche Vermögensgegenstände als liquide Mittel zu qualifizieren sind. ${ }^{68} \mathrm{Um}$ dem Ziel des neuen Tatbestands gerecht zu werden, dass eine Unternehmenskrise für eine erfolgreiche Durchführung einer Sanierung möglichst früh festgestellt wird, schlägt HACHMANN vor, dass vorerst nur die flüssigen Mittel, also jener Teil der liquiden Mittel, die äusserst geldnah sind, berücksichtigt werden sollten. ${ }^{69}$ Die in der Gesellschaft weiter vorhandenen, aber weniger geldnahen liquiden Mittel sollen erst berücksichtigt werden, sofern sie erwartungsgemäss zeitnah in flüssige Mittel umgewandelt werden sollen. ${ }^{70}$

Für eine Zahlungsunfähigkeit muss sowohl in zeitlicher als auch in quantitativer Hinsicht ein gewisses Mindestmass der mangelnden Liquidität vorliegen. ${ }^{71}$ Die einmalige Unmöglichkeit, fristgerecht zu zahlen, begründet noch keine

BBl 2017, 57 m.V.a. BGE 111 II 206 E. 1 S. 206.

HACHMANN, 101.

HACHMANN, 102.

HACHMANN, 102.

HACHMANN, 102.
} 
Zahlungsunfähigkeit. ${ }^{72}$ Für das Mindestausmass der drohenden Liquiditätslücke in quantitativer Hinsicht wird von HACHMANN propagiert, gleich wie bei Art. 190 Abs. 1 Ziff. 2 SchKG an die Gesamtsumme der Zahlungspflichten anzuknüpfen. In diesem Sinne wäre erst dann von einer Zahlungseinstellung auszugehen, wenn ein wesentlicher Anteil der gesamten fälligen Zahlungspflichten betroffen ist. ${ }^{73}$

Von einer drohenden Zahlungsunfähigkeit dürfte somit auszugehen sein, „wenn sich aufgrund qualifizierter innerer und äusserer Anzeichen die Hinweise verdichten, dass zukünftig die Zahlungsverpflichtungen nicht mehr erfüllt werden können “74 . Diese Definition überlässt dem Verwaltungsrat aber nach wie vor einen erheblichen Ermessensspielraum. Ein strengerer Massstab dürfte aber wohl bei besonderen, die Gesellschaft schwächenden Umständen (z.B. einer dünnen Liquiditätsausstattung) gelten. ${ }^{75}$

c) Handlungspflichten des Verwaltungsrats

aa) Im Allgemeinen

Der Verwaltungsrat muss sicherstellen, dass er eine drohende Zahlungsunfähigkeit jederzeit feststellen kann. ${ }^{76}$ Der neue nArt. 725 OR auferlegt dem Verwaltungsrat entsprechend zunächst die Pflicht, die Zahlungsfähigkeit der Gesellschaft zu überwachen. Die Überwachung der Liquiditätssituation ist bereits unter geltendem Recht eine dauernde Aufgabe des Verwaltungsrats, nachdem er im Rahmen der Finanzplanung die Liquiditätsplanung erstellt hat. ${ }^{77}$ In Nachachtung seiner Pflicht zur Ausgestaltung der Finanzplanung und Finanzkontrolle gemäss Art. 716a Abs. 1 Ziff. 3 OR hat er entsprechende prospektive Instrumente zur Überwachung der Liquidität zu implementieren ${ }^{78}$, welche Angaben zum Anfangsbestand an flüssigen Mitteln, zu den Planeinzahlungen und -auszahlungen sowie zum Planendbestand an flüssigen Mitteln

72

BBl 2017, 573 f. m.V.a. BGE 109 III 77 E. 2 S. 78 f.

HACHMANN, 103; BSK SCHKG II-BRUNNER/BOLLER, Art. 190, N 11.

HACHMANN, 104 f.; Erläuternder Bericht des EJPD vom 28. November 2014 zur Änderung des Obligationenrechts (Aktienrecht), Ziff. 2.1.33, S. 138.

HaChmann, 105; BöCKLI, Aktienrecht, § 13 N 767; BSK OR II-WÜstiner, Art. 725, N 32; SenN, 71; vgl. ferner MÜLLER/LIPP/PLÜSS, 2.

HaChManN, 105 f.; Simoniello, 542, wonach Finanzplanung über das reine Budgetieren hinaus insbesondere auch das Sicherstellen von genügend Liquidität zum richtigen Zeitpunkt bedeute; zu den Pflichten des Verwaltungsrats gemäss Art. 716a Abs. 1 Ziff. 3 OR ausführlich KäLIN, 325 ff.; vgl. ferner auch BBl 2017, 569.

GLANZMANN, Sanierung, 477; BöckLI, Aktienrecht, § 13 N 353.

HACHMANN, 106; SiMONIELLO, 543 ff. 
enthalten.$^{79}$ Unterlässt er dies, ist ihm dies gleichermassen vorzuwerfen, als würde er bei drohender Zahlungsunfähigkeit keine entsprechenden Massnahmen ergreifen. ${ }^{80}$ Durch die explizite Erwähnung dieser Aufgabe bei den sanierungsrechtlichen Bestimmungen könnte neu der falsche Eindruck erweckt werden, dass diese Überwachung nur in der Krise der Gesellschaft vorzunehmen ist. Dies ist jedoch nicht der Fall. ${ }^{81}$

Die vom Nationalrat ursprünglich vorgeschlagene Verpflichtung des Verwaltungsrats, die Zahlungsfähigkeit der Gesellschaft nicht nur zu überwachen, sondern auch zu gewährleisten, wurde vom Ständerat - u.E. zu Recht - nicht mitgetragen, hätte dies doch zu einer unsachlichen Erhöhung des Haftungsrisikos geführt. ${ }^{82}$

\section{bb) Bei drohender Zahlungsunfähigkeit}

Die weiteren Handlungspflichten des Verwaltungsrats knüpfen an die drohende Zahlungsunfähigkeit an und nicht erst an den Eintritt einer Zahlungsunfähigkeit. Wann eine drohende Zahlungsunfähigkeit vorliegt, wird vom Gesetz allerdings, wie dargelegt (vgl. oben, IV.2.b), nicht definiert. ${ }^{83}$

Droht die Zahlungsunfähigkeit einer Gesellschaft, muss der Verwaltungsrat die erforderlichen Massnahmen treffen, um die Zahlungsfähigkeit sicherzustellen. ${ }^{84}$ Er muss sodann, soweit erforderlich, weitere Massnahmen zur Sanierung der Gesellschaft ergreifen oder der Generalversammlung solche beantragen, soweit sie in deren Zuständigkeit fallen. ${ }^{85}$ Nötigenfalls muss er auch ein Gesuch um Nachlassstundung einreichen. ${ }^{86}$ Auf die ausdrückliche Nennung der Pflicht zur Erstellung eines Liquiditätsplans haben National- und Ständerat entgegen dem Vorschlag des Bundesrats verzichtet. Diese Korrektur ist zu begrüssen, da dies ohnehin selbstverständlich ist. ${ }^{87}$

Sanierungsmassnahmen sind zeitkritisch und schnelles Handeln ist geboten. Gemäss Botschaft wurde aber „[a]ufgrund unterschiedlichster Sachverhalte (Grösse des Unternehmens, Branche, Zugehörigkeit zu einem Konzern, Kom-

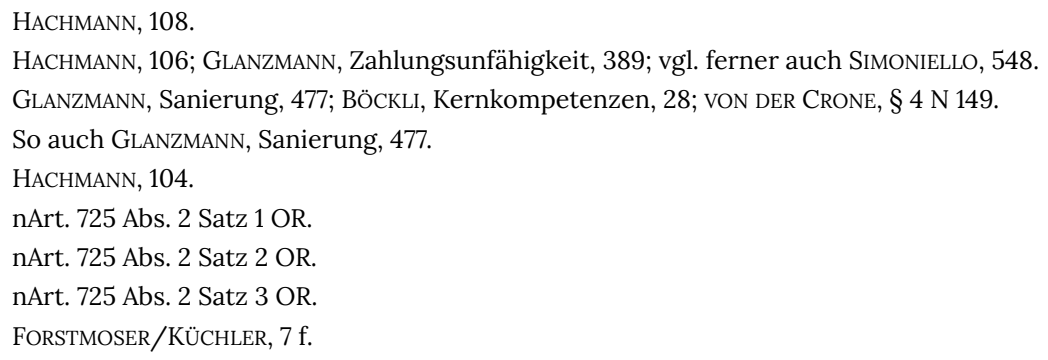


plexität der zu ergreifenden Massnahmen etc.) [...] darauf verzichtet, fixe Fristen für die Einhaltung der Handlungspflichten des Verwaltungsrats vorzuschreiben ${ }^{\text {“88 }}$. Stattdessen wird der Verwaltungsrat nach nArt. 725 Abs. 3 OR verpflichtet, „mit der gebotenen Eile“ zu handeln. Laut Botschaft kann dabei „an die vom Bundesgericht zum Konkursaufschub entwickelte Praxis angeknüpft werden, wonach dem [Verwaltungsrat] die nötige Zeit für die Erarbeitung von Sanierungsmassnahmen und gegebenenfalls deren Vorlage zuhanden der [Generalversammlung] zu gewähren ist, sofern begründete Aussicht auf wirksame und ausreichende Massnahmen besteht. Ansonsten aber besteht kein Raum für Verzögerungen und der [Verwaltungsrat] hat wie bisher unverzüglich zu handeln ${ }^{\text {“89 }}$. Gemäss Botschaft soll die in nArt. 725 Abs. 3 OR statuierte Pflicht im Falle einer späteren Überschuldung (vgl. nArt. 725b OR) insofern eine besondere Bedeutung haben, als deren Missachtung gegebenenfalls eine Verantwortlichkeit des Verwaltungsrats begründen könne. ${ }^{90}$

In Tat und Wahrheit scheint die explizite zeitliche Vorgabe des Handels mit der gebotenen Eile u.E. wenig griffig und führt kaum zu einer Verschärfung in zeitlicher Hinsicht. ${ }^{91}$

d) Was ändert sich?

Mit dem neuen Tatbestand der drohenden Zahlungsunfähigkeit sollte der Liquidität grössere Bedeutung beigemessen und ein eigentliches Frühwarnsystem eingeführt werden. ${ }^{92}$ Aus unserer Sicht ist allerdings fraglich, ob dieses Ziel erreicht wurde und dadurch eine effektive Erweiterung des Gläubigerschutzes und entsprechend mehr erfolgreiche Sanierungen ermöglicht werden.

Wie erwähnt, hat der Verwaltungsrat bereits gemäss geltendem Recht für eine Liquiditätsplanung und -überwachung zu sorgen. Gemäss Art. 716a Abs. 1 Ziff. 3 OR gehört die Finanzplanung zu den unübertragbaren und unentziehbaren Aufgaben des Verwaltungsrats. Eine Liquiditätsplanung ist dabei notwendiger Bestandteil jeder Finanzplanung. ${ }^{93}$

\footnotetext{
$88 \quad$ BBl 2017, 576.

89 BBl 2017, 576.

$90 \quad$ BBl 2017, 576.

91 GlanZMAnN, Sanierung, 478.

92 BBl 2017, 463.

93 Glanzmann, Sanierung, 467; GlanZmann, Zahlungsunfähigkeit, 391; BoEmLe/Stolz, 151; vgl. auch WALZ/GRAMLICH, 210, welche die Liquiditätsplanung als „Finanzplanung i.e.S.“ bezeichnen.
} 
Die Pflicht, die wirtschaftliche und finanzielle Lage der Gesellschaft kontinuierlich zu überwachen, ergibt sich auch aus der Aufgabe des Verwaltungsrats zur Oberleitung der Gesellschaft gemäss Art. 716a Abs. 1 Ziff. 1 OR. Ferner ist der Verwaltungsrat bereits heute aufgrund seiner Sorgfalts- und Treuepflicht nach Art. 717 OR verpflichtet, im Falle von finanziellen Problemen Sanierungsmassnahmen zu prüfen, vorzubereiten und durchzuführen. ${ }^{94}$

Zusammenfassend lässt sich somit zum neuen Tatbestand der drohenden Zahlungsunfähigkeit festhalten, dass dieser im Resultat nicht zu einer tiefgreifenden Änderung des Sanierungsrechts führen dürfte. Es handelt sich vielmehr um eine Konkretisierung resp. Kodifizierung des bereits geltenden Rechts. ${ }^{95}$ Auch in zeitlicher Hinsicht wird keine maximale Toleranzfrist für das Vorgehen des Verwaltungsrats vorgesehen, sondern mit der Vorgabe des Handelns mit der gebotenen Eile an einer flexiblen Handhabung basierend auf den besonderen Verhältnissen des Einzelfalls festgehalten.

\section{Kapitalverlust}

\section{a) Übersicht}

Der Kapitalverlust wird neu in einem eigenständigen Artikel geregelt, nämlich in nArt. 725a OR. Das heutige Konzept wird aber mit ein paar Modifikationen beibehalten.

Der neue Tatbestand des Kapitalverlusts gemäss nArt. 725a OR wird wie folgt lauten:

${ }^{1}$ Zeigt die letzte Jahresrechnung, dass die Aktiven abzüglich der Verbindlichkeiten die Hälfte der Summe aus Aktienkapital, nicht an die Aktionäre zurückzahlbarer gesetzlicher Kapitalreserve und gesetzlicher Gewinnreserve nicht mehr decken, so ergreift der Verwaltungsrat Massnahmen zur Beseitigung des Kapitalverlusts. Er trifft, soweit erforderlich, weitere Massnahmen zur Sanierung der Gesellschaft oder beantragt der Generalversammlung solche, soweit sie in deren Zuständigkeit fallen.

${ }^{2}$ Hat die Gesellschaft keine Revisionsstelle, so muss die letzte Jahresrechnung vor ihrer Genehmigung durch die Generalversammlung überdies einer eingeschränkten Revision durch einen zugelassenen Revisor unterzogen werden. Der Verwaltungsrat ernennt den zugelassenen Revisor.

94 SPRECHER/SOMmER, Sanierung, 552; BSK OR II-WÜSTINER, Art. 725, N 32.

95 So auch HaCHMAnN, 117; SimONiELlO, 548. 
${ }^{3}$ Die Revisionspflicht nach Absatz 2 entfällt, wenn der Verwaltungsrat ein Gesuch um Nachlassstundung einreicht.

${ }^{4}$ Der Verwaltungsrat und die Revisionsstelle oder der zugelassene Revisor handeln mit der gebotenen Eile.

\section{b) Begriff „Kapitalverlust“}

In Abweichung zum hälftigen Kapitalverlust nach geltendem Recht hätte nach dem ursprünglichen Vorschlag des Bundesrats ein Kapitalverlust bereits dann vorliegen sollen, wenn die Aktiven abzüglich der Verbindlichkeiten zwei Drittel der Summe aus Aktienkapital und gewisser Reserven nicht mehr decken. ${ }^{96}$ Dies hätte zur Folge haben können, dass sich gewisse neu gegründete Gesellschaften schon kurz nach ihrer Entstehung mit Sanierungsmassnahmen hätten auseinandersetzen müssen. Denn praxisgemäss fällt zu Beginn einer Geschäftstätigkeit zunächst vor allem Aufwand an. National- und Ständerat haben sich daher entschieden, wie unter geltendem Recht beim hälftigen Kapitalverlust zu bleiben (vgl. oben, III.2.). ${ }^{97}$

Neu wird klargestellt, dass nur der geschützte Teil der gesetzlichen Reserven als Bemessungsbasis dient. In dieser Frage erfolgt somit ein Anschluss an die heutige Minderheitsmeinung. ${ }^{98}$

\section{c) Handlungspflichten des Verwaltungsrats}

Die Handlungspflichten des Verwaltungsrats werden im Vergleich zum geltenden Gesetzestext leicht modifiziert und zwar dahingehend, dass der Verwaltungsrat bei Vorliegen eines Kapitalverlusts primär Massnahmen zu dessen Beseitigung ergreifen muss. ${ }^{99}$ Weitere Massnahmen zur Sanierung der Gesellschaft sind nur bei entsprechender Erforderlichkeit vorzunehmen. ${ }^{100}$ Diese neue Kaskade führt dazu, dass ein Kapitalverlust zunächst durch reine Bilanzkosmetik beseitigt werden kann, ohne dass die Gesellschaft Sanierungsmassnahmen zur effektiven Eigenkapitalvergrösserung ergreift. ${ }^{101}$

Auf die unter dem geltenden Recht vorgesehene zwingende Einberufung einer sog. Sanierungsversammlung wird künftig verzichtet. Eine Sanierungsver-

\footnotetext{
$96 \quad$ BBl 2017, 576.

97 BBl 2020, 5609 f.; FORSTMOSER/KÜCHLER, 8.

98 GlanZmanN, Sanierung, 478; BBI 2017, 577 m.V.a. ZK OR-HOMBURGER, Art. 725, N 1204 ff.; BSK OR II-WÜSTINER, Art. 725, N 18.

99 nArt. 725a Abs. 1 Satz 1 OR; GLANZMANN, Sanierung, 478; vgl. dazu auch oben, III.3.

100 nArt. 725a Abs. 1 Satz 2 OR.

101 GLANZMANN, Sanierung, 469.
} 
sammlung ist nur einzuberufen, wenn Sanierungsmassnahmen vorgenommen werden sollen, die einen Beschluss der Generalversammlung voraussetzen. ${ }^{102}$ Diese Neuerung ist u.E. zu begrüssen. Die unter geltendem Recht gesetzlich vorgesehene unverzügliche Einberufung einer Generalversammlung dürfte oft eher kontrapoduktiv als zielführend sein. Insbesondere in grösseren Verhältnissen hat die Einberufung einer Generalversammlung die Publikmachung der finanziellen Probleme zur Folge. ${ }^{103}$ Dies kann die Lage für die Gesellschaft noch verschlimmern, wenn etwa aufgrund der bekannt gewordenen finanziellen Krise die Lieferanten der Gesellschaft dazu übergehen, Lieferungen nur noch gegen Vorauskasse zu leisten. Ein solches Szenario führt häufig umgehend zur Illiquidität der Gesellschaft. ${ }^{104}$

Nach der neuen gesetzlichen Konzeption entfällt bei Eintritt eines Kapitalverlusts ferner die Berechtigung zum Opting-out. Hat die Gesellschaft keine Revisionsstelle, so muss gemäss nArt. 725a Abs. 2 OR die letzte Jahresrechnung vor ihrer Genehmigung durch die Generalversammlung einer eingeschränkten Revision durch einen zugelassenen Revisor unterzogen werden. Der Verwaltungsrat hat den zugelassenen Revisor zu ernennen. Falls der Verwaltungsrat diese Prüfung unterlässt, sind die Generalversammlungsbeschlüsse zur Genehmigung der Jahres- und Konzernrechnung sowie zur Verwendung des Bilanzgewinns grundsätzlich nichtig (Art. 731 Abs. 3 OR). ${ }^{105}$ Wie sich aus nArt. 725a Abs. 3 OR ergibt, ist die Gesellschaft von der Revisionspflicht jedoch befreit, wenn der Verwaltungsrat ein Gesuch um Nachlassstundung einreicht.

Auch beim Tatbestand des Kapitalverlusts wird explizit festgehalten, dass der Verwaltungsrat und die Revisionsstelle bzw. unter Umständen der zugelassene Revisor mit der gebotenen Eile handeln müssen. ${ }^{106}$ Wie bereits erwähnt, ist diese Vorgabe allerdings wenig griffig. ${ }^{107}$

\section{d) Was ändert sich?}

Wie eben ausgeführt, wird der Kapitalverlust neu in einem eigenständigen Gesetzesartikel geregelt. Eine grundlegende Änderung, welche die Praxisrelevanz des Kapitalverlusts erhöhen würde, erfährt die revidierte Regelung des Kapitalverlusts u.E. aber nicht. Neu werden Massnahmen zur Beseitigung des

\footnotetext{
102 nArt. 725a Abs. 1 Satz 2 OR; GlanZMANN, Sanierung, 478.

103 Glanzmann, Sanierung, 469 f.; Buser, N 43 f., zur Frage, wann potentiell kursrelevante Tatsachen von der Adhoc-Publizität vorübergehend ausgenommen werden dürfen.

104 GlanzmanN, Sanierung, 469; vgl. auch BÖCKLI, Aktienrecht, § 13 N 754 und 754b.

105 Vgl. zum Ganzen GLanZMANN, Sanierung, 478.

106 nArt. 725a Abs. 4 OR.

107 Vgl. dazu oben, IV.2.c; vgl. auch GLANZMANN, Sanierung, 478.
} 
Kapitalverlusts gar als primäre Pflicht des Verwaltungsrats deklariert, weshalb das Risiko, dass ein Kapitalverlust durch reine Bilanzkosmetik beseitigt wird und Sanierungsmassnahmen gar nicht oder verspätet implementiert werden, noch erhöht werden dürfte. ${ }^{108}$

Ausserdem kann wohl nicht ausgeschlossen werden, dass bei Gesellschaften mit einem Opting-out in vielen Fällen ein Kapitalverlust und der entsprechende Wegfall der Berechtigung zum Opting-out unbemerkt bleibt. ${ }^{109}$ Positiv zu werten ist der Wegfall der Pflicht des Verwaltungsrats zur Einberufung einer Sanierungsversammlung. Die Sanierungsversammlung hat in der Praxis - wie erwähnt - in vielen Fällen, wenn überhaupt, nur einen geringen Nutzen und durch deren Weglassen kann im Rahmen einer effektiv an die Hand genommenen Sanierung unter Umständen wertvolle Zeit eingespart werden. ${ }^{110}$

\section{4. Überschuldung}

\section{a) Übersicht}

Die dritte Säule des aktienrechtlichen Sanierungsrechts bildet der Tatbestand der Überschuldung, welcher neu mit nArt. 725b OR in einem eigenständigen Artikel geregelt sein wird.

Die neue Gesetzesbestimmung zur Überschuldung gemäss nArt. 725b OR wird wie folgt lauten:

${ }^{1}$ Besteht begründete Besorgnis, dass die Verbindlichkeiten der Gesellschaft nicht mehr durch die Aktiven gedeckt sind, so erstellt der Verwaltungsrat unverzüglich je einen Zwischenabschluss zu Fortführungswerten und Veräusserungswerten. Auf den Zwischenabschluss zu Veräusserungswerten kann verzichtet werden, wenn die Annahme der Fortführung gegeben ist und der Zwischenabschluss zu Fortführungswerten keine Überschuldung ausweist. Ist die Annahme der Fortführung nicht gegeben, so genügt ein Zwischenabschluss zu Veräusserungswerten.

${ }^{2}$ Der Verwaltungsrat lässt die Zwischenabschlüsse durch die Revisionsstelle oder, wenn eine solche fehlt, durch einen zugelassenen Revisor prüfen; er ernennt den zugelassenen Revisor. Fehlkonzeption dieses Tatbestands beibehalten und teilweise sogar noch verstärkt wird“. 
${ }^{3}$ Ist die Gesellschaft gemäss den beiden Zwischenabschlüssen überschuldet, so benachrichtigt der Verwaltungsrat das Gericht. Dieses eröffnet den Konkurs oder verfährt nach Artikel 173a des Bundesgesetzes vom 11. April 1989 über Schuldbetreibung und Konkurs.

${ }^{4}$ Die Benachrichtigung des Gerichts kann unterbleiben:

1. wenn Gesellschaftsgläubiger im Ausmass der Überschuldung im Rang hinter alle anderen Gläubiger zurücktreten und ihre Forderungen stunden, sofern der Rangrücktritt den geschuldeten Betrag und die Zinsforderungen während der Dauer der Überschuldung umfasst; oder

2. solange begründete Aussicht besteht, dass die Überschuldung innert angemessener Frist, spätestens aber 90 Tage nach Vorliegen der geprüften Zwischenabschlüssen, behoben werden kann und dass die Forderungen der Gläubiger nicht zusätzlich gefährdet werden.

${ }^{5}$ Verfügt die Gesellschaft über keine Revisionsstelle, so obliegen dem zugelassenen Revisor die Anzeigepflichten der eingeschränkt prüfenden Revisionsstelle.

${ }^{6}$ Der Verwaltungsrat und die Revisionsstelle oder der zugelassene Revisor handeln mit der gebotenen Eile.

\section{b) Begriff „Überschuldung“}

Der Begriff der Überschuldung bleibt unter dem revidierten Sanierungsrecht unverändert, weshalb für die Begrifflichkeit auf oben III.2 verwiesen werden kann.

\section{c) Handlungspflichten des Verwaltungsrats}

aa) Bei begründeter Besorgnis einer Überschuldung

Wie bisher knüpfen die Handlungspflichten des Verwaltungsrats vorerst am Vorliegen einer begründeten Besorgnis einer Überschuldung an. Zentral bleibt damit, dass der Verwaltungsrat durch eine geeignete Ausgestaltung des Rechnungswesens und der Finanzkontrolle sicherzustellen hat, dass er eine begründete Besorgnis einer Überschuldung erlangen kann, um seinen entsprechenden Pflichten nachzukommen. ${ }^{11}$

\footnotetext{
111 Vgl. dazu oben, IV.2.c.
} 
Neu wird klargestellt, dass bei begründeter Besorgnis einer Überschuldung nicht nur wie nach dem bisherigen Wortlaut des Gesetzes eine Zwischenbilanz, sondern ein Zwischenabschluss zu erstellen ist, wozu eine Bilanz, eine Erfolgsrechnung und ein Anhang gehören. ${ }^{12}$ Dies entspricht wohl bereits der heutigen h.L. ${ }^{113}$ In zeitlicher Hinsicht wird ebenfalls präzisiert, dass der Verwaltungsrat hierbei unverzüglich handeln muss. ${ }^{114}$

Neu wird im Gesetz festgehalten, dass auf die Erstellung eines Zwischenabschlusses zu Veräusserungswerten verzichtet werden kann, wenn die Annahme der Fortführung gegeben ist und der Zwischenabschluss zu Fortführungswerten keine Überschuldung ausweist. ${ }^{115}$ Ist die Annahme der Fortführung nicht gegeben, so genügt ein Zwischenabschluss zu Veräusserungswerten. ${ }^{116}$ Damit wird die heute von der Rechtsprechung akzeptierte Praxis bezüglich der Notwendigkeit der Erstellung eines Zwischenabschlusses zu Veräusserungswerten in das Gesetz überführt. ${ }^{117}$

Klargestellt wird mit nArt. 725b OR schliesslich, dass der Verwaltungsrat die Zwischenabschlüsse durch die Revisionsstelle der Gesellschaft prüfen lassen muss. Dies entspricht weitgehend der heutigen Praxis. ${ }^{118}$ Bei Gesellschaften, die über keine Revisionsstelle verfügen, hat die Prüfung durch einen zugelassenen Revisor zu erfolgen, der vom Verwaltungsrat zu ernennen ist. ${ }^{119}$

\section{bb) Bei Überschuldung}

Ist die Gesellschaft überschuldet, hat der Verwaltungsrat wie bisher grundsätzlich das Gericht zu benachrichtigen und die Konkurseröffnung zu beantragen oder um Gewährung einer Nachlassstundung zu ersuchen..$^{120}$ Die Gewährung eines Konkursaufschubs ist nicht mehr möglich. Der Konkursaufschub ist primär dafür geeignet, eine stille Sanierung in einem geschützten Rahmen

\footnotetext{
112 nArt. 725b Abs. 1 i.V.m. nArt. 960f Abs. 1 OR.

113 GlanZmanN, Sanierung, 478 f.; BÖCKLI, Aktienrecht, § 13 N 770 f. und 819; BSK OR II-WÜSTINER, Art. 725, N 36. 
durchzuführen. Er ist in der Praxis wenig verbreitet. ${ }^{121}$ Vor dem Hintergrund, dass die provisorische (auch stille) Nachlassstundung neu auf bis acht Monate erstreckbar sein wird, wird der Konkursaufschub im Rahmen des Überschuldungstatbestands abgeschafft. ${ }^{122}$

Nach der gesetzlichen Konzeption erfolgt die Bilanzdeponierung beim Gericht somit - wie unter geltendem Recht - erst in einem Zeitpunkt, in welchem die Gläubiger meist bereits einen erheblichen Schaden erlitten haben. Dies gilt selbst bei pflichtgemässem Verhalten des Verwaltungsrats. ${ }^{123}$

Die Konkurseröffnung dürfte nach wie vor die wohl am häufigsten ergriffene Massnahme bei eingetretener Überschuldung sein. ${ }^{124}$ Das Konkursverfahren ist aber kein Sanierungsverfahren, vielmehr führt es in der Regel zum endgültigen Untergang des Unternehmens. Der Geschäftsbetrieb kann meistens nicht weitergeführt werden, weil der an die Stelle der bisherigen Organe tretende Konkursverwalter nur selten willens und in der Lage ist, das Geschäft fortzuführen. Zudem sind Veräusserungen aus der Konkursmasse in aller Regel sehr schwerfällig. Folge davon ist, dass die in einem Konkurs erzielbaren Werte besonders tief sind und die Gläubiger der dritten Klasse regelmässig lediglich mit einer Konkursdividende im einstelligen Prozentbereich rechnen können. ${ }^{125}$

Das Nachlassverfahren ist demgegenüber grundsätzlich ein gutes Sanierungsverfahren. Im Nachlassverfahren kann der Geschäftsbetrieb aufrechterhalten werden. Sodann wird im Nachlassverfahren ein Neuanfang ermöglicht, weil die Nachlassstundung zwischen Nachlassforderungen und privilegierten Masseverbindlichkeiten trennt. ${ }^{126}$ Für kleine Verhältnisse ist das Nachlassverfahren aber zumeist keine valable Alternative, da es relativ beratungsintensiv und damit oft mit hohen Kosten verbunden ist. ${ }^{127}$

Wie unter geltendem Recht kann im Überschuldungsfall von der Benachrichtigung des Gerichts abgesehen werden, wenn Rangrücktritte von Gesellschaftsgläubigern vorliegen, welche die Überschuldung abdecken. ${ }^{128}$ Darauf wird nachfolgend noch eingegangen (vgl. dazu im Detail unten, IV.4.c.dd). Weiter kann auf eine Überschuldungsanzeige verzichtet werden, wenn der

\footnotetext{
121 GlanZmanN, Sanierung, 475.

122 nArt. 293a Abs. 2 SchKG; vgl. zum Ganzen GlanZMANN, Sanierung, 476; BBl 2017, 465.

123 GLANZMANN, Sanierung, 474.

124 GlanZMANN, Sanierung, 474.

125 GlanZMANN, Sanierung, 474.

126 GlanZmann, Sanierung, 474; GlanZmanN, Sanierungsrecht, $248 \mathrm{ff}$.

127 GlanZMANN, Sanierung, 475.

128 nArt. 725b Abs. 4 Ziff. 1 OR.
} 
Verwaltungsrat die Gesellschaft sanieren kann. ${ }^{129}$ Nach bundesgerichtlicher Rechtsprechung ist dies auch unter geltendem Recht zulässig. ${ }^{130}$ Nun soll dieser Tatbestand der stillen Sanierung explizit kodifiziert werden (vgl. dazu im Detail unten, IV.4.c.ee).

\section{cc) Vorgaben in zeitlicher Hinsicht}

Neu wird in zeitlicher Hinsicht festgehalten, dass der Verwaltungsrat bei begründeter Besorgnis einer Überschuldung unverzüglich einen Zwischenabschluss erstellen muss und im Übrigen mit der gebotenen Eile zu handeln hat. ${ }^{131}$ Auf die gesetzliche Fixierung einer maximalen allgemeinen Toleranzfrist wurde somit verzichtet. Ab wann den Organen im Rahmen von aktienrechtlichen Verantwortlichkeitsklagen eine Konkursverschleppung vorgeworfen werden kann, bleibt damit - wie unter geltendem Recht - eine Frage, die in jedem Einzelfall anhand der konkreten Umstände zu prüfen sein wird. Nur in Bezug auf die stille Sanierung wurde eine Maximalfrist vorgesehen, welche das Haftungsrisiko für den Verwaltungsrat gegebenenfalls eindämmen könnte (vgl. dazu unten, IV.4.c.ee).

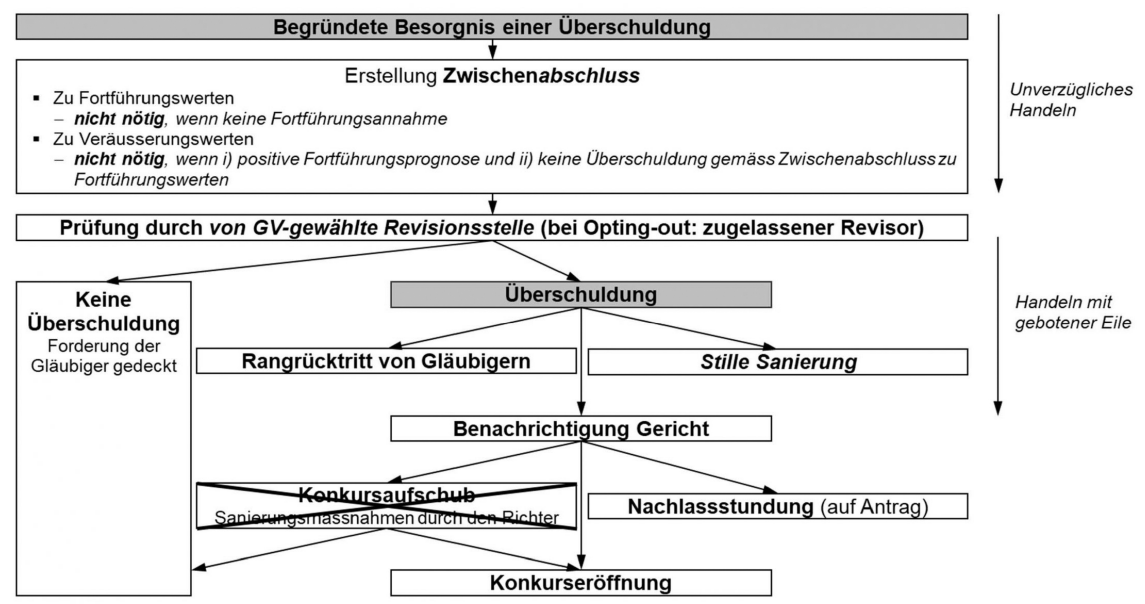

nArt. 725b Abs. 4 Ziff. 2 OR.

130 Vgl. dazu oben, III.4.b.

131 nArt. 725b Abs. 6 OR. Gleiches gilt auch für die Revisionsstelle oder den zugelassenen Revisor. 


\section{dd) Rangrücktritt}

Wie eben erwähnt, kann die Benachrichtigung des Gerichts unterbleiben, wenn Gesellschaftsgläubiger im Ausmass der Überschuldung im Rang hinter alle anderen Gläubiger zurücktreten und ihre Forderungen stunden. ${ }^{132}$

Unter geltendem Recht können die im Rang zurücktretenden Gläubiger bis zur Insolvenz weiterhin die fälligen Zinsen einfordern. Damit wird der Gesellschaft indessen keine umfassende Erleichterung verschafft. ${ }^{133}$ Neu gelten daher erhöhte Anforderungen an einen Rangrücktritt: Die Stundung muss künftig nicht nur wie bisher den geschuldeten Betrag, sondern auch die Zinsforderungen umfassen. Ob diese Verschärfung - die im Widerspruch zur aktuellen Rechtsprechung des Bundesgerichts steht ${ }^{134}$ - dazu führen wird, dass seitens der Gläubiger weniger oft Rangrückritte erklärt werden, wird die Praxis zeigen. Gleichsam wird sich zeigen, ob die Abgrenzung von Zinsen zu übrigen Entschädigungen zu Diskussionen führen wird; für Letztere verlangt das Gesetz keine Stundung. ${ }^{135}$

Die vom Nationalrat vorgeschlagene weitere massive Erschwerung des Rangrücktritts, wonach ein Rangrücktritt nur noch bei bestehender Aussicht, dass die Gesellschaft saniert werden kann, von der Pflicht zur Benachrichtigung des Richters befreien sollte ${ }^{136}$ wurde vom Ständerat zu Recht abgelehnt. Bislang war mit dem Rangrücktritt die positive Eigenschaft verbunden, dass eine Überschuldungssituation schnell und effektiv unter Kontrolle gebracht werden konnte. Durch die vom Nationalrat vorgeschlagene Erschwerung wäre der Rangrücktritt dieser positiven Eigenschaft beraubt worden. Zudem hätte diese Regelung eine gewisse Rechtsunsicherheit für den Verwaltungsrat und damit letztlich ein erhöhtes Haftungsrisiko für diesen mit sich gebracht. ${ }^{137}$

Im Zusammenhang mit Rangrücktrittsforderungen ist ferner zu erwähnen, dass das Parlament mit nArt. 757 Abs. 4 OR die Rechtsprechung des Bundes-

\footnotetext{
132 nArt. 725b Abs. 4 Ziff. 1 OR; zum Begriff des Rangrücktritts vgl. oben, IV.4.c.

133 SPReCher/SOMmer, Sanierung, 556; BÖCKLI, Aktienrecht, § 13 N 793; BÜHLER, Aktiengesellschaft, 447; ForstMOSER/MEIER-HAYOZ/NOBEL, § 50 N 215 ff.; vgl. oben, IV.4.c.

134 Urteil des Bundesgerichts 4C.47/2003 vom 2. Juli 2003 E. 4.

135 Vgl. zum Ganzen Glanzmann, Sanierung, 479; Glanzmann, Zahlungsunfähigkeit, 400.

136 Vom Nationalrat vorgeschlagene Fassung: „[...] wenn Gesellschaftsgläubiger im Ausmass der Überschuldung im Rang hinter alle anderen Gläubiger zurücktreten und ihre Forderungen stunden, sofern der Rangrücktritt den geschuldeten Betrag und die Zinsforderungen während der Dauer der Überschuldung umfasst und die Aussicht besteht, dass die Gesellschaft saniert werden kann; [...]."

137 GlanzmanN, Sanierung, 479.
} 
gerichts, wonach solche Forderungen Teil des Gesellschaftsschadens sind ${ }^{138}$, korrigiert hat. ${ }^{139}$ Gemäss nArt. 757 Abs. 4 OR sind Forderungen von Gesellschaftsgläubigern, die im Rang hinter alle anderen Gläubiger zurückgetreten sind, nicht in die Berechnung des Schadens der Gesellschaft einzubeziehen. Damit soll einerseits die Sanierungsmassnahme des Rangrücktritts erleichtert und andererseits das damit verbundene Haftungsrisiko des Verwaltungsrats reduziert werden. ${ }^{140}$ Der Nationalrat wollte zunächst auf die entsprechende Gesetzesänderung verzichten, stimmte ihr aber letztlich doch zu.

\section{ee) Stille Sanierung}

Wie bereits angesprochen, ist es unter geltender Praxis zulässig, die Benachrichtigung des Gerichts aufzuschieben, um Erfolg versprechende Sanierungshandlungen (im Sinne einer sog. stillen Sanierung) zu ermöglichen. Die Dauer des Aufschubs ist - wie der Aufschub selbst - nicht gesetzlich geregelt. ${ }^{141}$

Das künftige Recht spricht dies nun $\mathrm{an}^{142}$ : Die stille Sanierung wird im Wesentlichen der heutigen bundesgerichtlichen Praxis entsprechend ins Gesetz überführt. ${ }^{143}$ Zwei wichtige Punkte waren in der parlamentarischen Beratung aber lange umstritten:

Unter geltendem Recht halten es das Bundesgericht ${ }^{144}$ und die wohl h.L. ${ }^{145}$ für zulässig, dass der Verwaltungsrat die Benachrichtigung des Gerichts für eine verhältnismässig kurze Zeitspanne von vier bis sechs Wochen aufschiebt und Sanierungsmassnahmen versucht. ${ }^{146}$ Ob bei guten Sanierungsaussichten auch ein längerer Aufschub zulässig ist, ist umstritten. ${ }^{147}$ Während diesbezüglich der Ständerat (wie der Bundesrat) mittels einer maximalen Toleranzfrist Rechtssicherheit schaffen und einen Aufschub von maximal 90 Tagen zulassen

\footnotetext{
138 Urteil des Bundesgerichts 4A.277/2010 vom 2. September 2010 E. 2.3.

139 GlanZmann, Sanierung, 480; GLANZMAnN/Wolf, $27 \mathrm{f}$.

140 Glanzmann, Sanierung, 480.

141 Vgl. dazu oben, III.4.b.

142 nArt. 725b Abs. 4 Ziff. 2 OR.

143 GLANZMAnN, Sanierung, 480.

144 BGE 76 I 162 E. 3 S. 167; BGE 108 V 183 E. 2 S. 188; BGE 116 II E. 5a S. 541; BGE 132 III 564 E. 5.1

S. 573; Urteil des Bundesgerichts 4C.436/2006 vom 18. April 2007 E. 4 ff.

145 Vgl. statt vieler: BÖCKLI, Aktienrecht, § 13 N 816.

146 SpReCher/SOMmer, Sanierung, 555; vgl. die umfassende Zusammenstellung von Rechtsprechung und Doktrin in FORSTMOSER, Krisenmanager, 369 ff. und 372 ff.; MEIER-HaYOZ/FORSTMOSER/SETHE, § 16 N 131.

147 Bejahend FORSTMOSER, Krisenmanager, 280 f.; verneinend BÖCKLI, Aktienrecht, § 13 N 777 und 817h ff.; vgl. zum Ganzen auch SPRECHER/SOMMER, Sanierung, 555.
} 
wollte ${ }^{148}$, erachtete der Nationalrat eine „kurze, den Umständen angemessene Frist $^{\text {“149 }}$ als zielführend. Der Vorschlag des Nationalrats vermochte sich nicht durchzusetzen. Für die stille Sanierung gilt somit neu eine kodifizierte Maximalfrist von 90 Tagen.

In Bezug auf die Voraussetzungen des Aufschubs konnte sich der Nationalrat hingegen durchsetzen: Nach dem Vorschlag des Bundesrats (dem der Ständerat gefolgt ist) hätte sich in der Sanierungsphase die Überschuldung „nicht wesentlich [erhöhen] “"150 dürfen. Gemäss dem Vorschlag des Nationalrats, der sich durchsetzen konnte, dürfen hingegen während der Sanierungsbemühungen „die Forderungen der Gläubiger nicht zusätzlich gefährdet werden “"151.

\section{d) Was ändert sich?}

Der Tatbestand der Überschuldung wird wohl auch zukünftig in der Praxis zentral bleiben. Neu wird er in einem eigenständigen Artikel geregelt. Wie der Kapitalverlust erfährt auch der Tatbestand der Überschuldung konzeptionell keine tiefgreifenden Änderungen. ${ }^{152}$ Neu wird der Tatbestand der Überschuldung durch eine maximale Toleranzfrist von 90 Tagen zur Durchführung einer stillen Sanierung ergänzt. Damit wird gegenüber dem geltenden Recht der Handlungsspielraum des Verwaltungsrats in zeitlicher Hinsicht konkretisiert und entsprechend Rechtssicherheit geschaffen. Aus Sicht des Verwaltungsrats ist diese klare zeitliche Vorgabe sicherlich zu begrüssen. Aus Sicht der Gesellschaft ist aber fraglich, ob diese Maximalfrist in jedem Einzelfall die nötige flexible Handhabung ermöglicht oder teilweise auch zu kurz oder zu lang bemessen sein könnte.

\footnotetext{
148 Vom Ständerat vorgeschlagene Fassung: „[...] solange begründete Aussicht besteht, dass die Überschuldung innert angemessener Frist, spätestens aber 90 Tage nach Vorliegen der geprüften Zwischenbilanzen, behoben werden kann und dass sich die Überschuldung nicht wesentlich erhöht."

Vom Nationalrat vorgeschlagene Fassung: „[...] wenn die begründete Aussicht besteht, dass die Überschuldung innert kurzer, den Umständen angemessener Frist behoben und die Gesellschaft saniert werden kann und dass die Forderungen der Gläubiger nicht zusätzlich gefährdet werden."; vgl. zum Ganzen auch FORSTMOSER/KüCHLER, 8.

150 Vgl. oben, Fn. 149; vgl. ferner FoRSTMOSER/KÜCHLER, 8

151 Vgl. oben, Fn. 150; vgl. ferner ForSTMOSER/KüCHLER, 8.

152 GlanZMAnN, Sanierung, 478.
} 


\section{Sanierungsmassnahmen}

Es wurde gezeigt, dass der Verwaltungsrat unter dem neuen Aktienrecht bei drohender Zahlungsunfähigkeit, Kapitalverlust und Überschuldung handeln muss. Noch nicht näher beleuchtet wurde die Frage, wie der Verwaltungsrat gemäss der neuen gesetzlichen Konzeption bei der eigentlichen Sanierung vorgehen soll. Dieser Frage wollen wir uns der Vollständigkeit halber noch annehmen, wobei die einzelnen Sanierungsmassnahmen nur generisch erwähnt werden.

Die neuen Bestimmungen im OR zum Sanierungsrecht verwenden die Begrifflichkeiten i) „Massnahmen zur Sicherstellung der Zahlungsfähigkeit ${ }^{\text {“153 }}$, ii) „Massnahmen zur Beseitigung des Kapitalverlusts“"154 sowie iii) „weitere Massnahmen zur Sanierung der Gesellschaft ${ }^{\text {" }}{ }^{155}$. Weder im geltenden Recht noch in den neuen Bestimmungen wird definiert, was unter diesen Massnahmen zu verstehen ist. Sie werden nachfolgend der Einfachheit halber zusammengefasst als „Sanierungsmassnahmen“ bezeichnet.

Gemäss bundesgerichtlicher Rechtsprechung werden unter Sanierung „sämtliche Massnahmen verstanden, die auf die finanzielle Gesundung der Gesellschaft [...], d.h. auf den Fortbestand der Gesellschaft und die Verhinderung der Liquidation abzielen [...]. Im Falle einer Überschuldung ist das kurzfristige Ziel von Sanierungsmassnahmen, die Gesellschaft mindestens in einen Zustand zu versetzen, dass die Anrufung des Richters nach Art. 725 Abs. 2 OR unabhängig von allfälligen Rangrücktrittserklärungen vermieden werden kann “156. In der Lehre wird der Begriff "Sanierungsmassnahmen“ dahingehend verwendet, als dass darunter „alle finanziellen, rechtlichen und betrieblichen Massnahmen $\mathrm{zu}$ verstehen sind, die auf die Beseitigung einer Zahlungsunfähigkeit, eines Kapitalverlusts oder einer Überschuldung sowie auf die Wiederherstellung der Rentabilität eines Unternehmens zielen “157. Sanierungsmassnahmen sind damit primär auf eine Ertragssteigerung, Liquiditätssicherung und Bilanzverbesserung ausgerichtet. ${ }^{158}$

nArt. 725 Abs. 2 OR.

nArt. 725a Abs. 1 OR.

nArt. 725a Abs. 1 OR.

BGE 138 III 204 E. 3.3.1 S. 209 f.

DUBACH, $53 \mathrm{f}$.

SPRECHER/SOMmer, Sanierung, 552; SCHENKER, 488; KeSSELBACH, $30 \mathrm{ff}$. 


\section{Bilanzierungsmassnahmen}

Im Sinne einer bilanztechnischen Massnahme kann die Gesellschaft durch die Auflösung von stillen Reserven ihr Bilanzbild verbessern. Als weitere Bilanzierungsmassnahme gilt auch die Aufwertung von Grundstücken oder Beteiligungen (vgl. unten, V.4). Beide bilanzrechtlichen Möglichkeiten fallen in die Kompetenz des Verwaltungsrats. ${ }^{159}$

\section{2. $\quad$ Änderung der Kapitalstruktur / Liquiditätsverbesserung}

Neben Bilanzierungsmassnamen sind auch Massnahmen unter Einbezug der Aktionäre und Gläubiger denkbar, welche auf eine Kapitalstrukturänderung oder Liquiditätsverbesserung abzielen. ${ }^{160}$

Als Massnahmen unter Einbezug der Aktionäre zu nennen sind z.B. eine Kapitalerhöhung, eine Kapitalherabsetzung oder ein Kapitalschnitt (sog. „Harmonika“) ${ }^{161}$. Auch das neue Instrument des Kapitalbands gemäss nArt. 653s ff. OR ermöglicht insbesondere aufgrund der damit einhergehenden Möglichkeit zur Kapitalherabsetzung ein rascheres Vorgehen des Verwaltungsrats und verbessert die Sanierungsmöglichkeiten ausserhalb eines Nachlassverfahrens. ${ }^{162}$ In engem Zusammenhang mit der Sanierung im OR steht ferner die Möglichkeit zur Verrechnungsliberierung mit nicht werthaltigen Forderungen, welche im neuen Aktienrecht in nArt. 634a OR kodifiziert wird. ${ }^{163}$

Als Massnahmen unter Einbezug der Gläubiger kommen namentlich Stillhalteabkommen, in welchen Gläubiger für eine bestimmte Zeitspanne auf ihre Rückforderungen verzichten, Überbrückungskredite, Forderungsverzichte oder Rangrücktritte in Frage. ${ }^{164}$

\section{Operationelle Massnahmen}

Im Sinne einer operationellen Massnahme können zur Erhöhung der Liquidität nicht betriebsnotwendige Aktiven wie Liegenschaften oder Unternehmensbe-

Teitler-Feinberg/Lipp, 386 f. und 390; SPRecher/Sommer, Sanierung, 552; Schenker, 488; vgl. zur Bilanzbereinigung auch BöCKLI, Aktienrecht, § $13 \mathrm{~N} 716$ und 758.

Vgl. für eine Übersicht auch SPRECher/SOMmer, Sanierung, 553 f. und Teitler-FeinberG/ LIPP, 387.

Vgl. nArt. 653q f. OR; BBl 2017, 511 ff.

Zu einer Übersicht der neuen Regelung des Kapitalbands vgl. FORSTMOSER/KÜCHLER, 5 f.; BBl 2017, 513 ff.

BBl 2017, 463.

Vgl. SPRECHER/SOMMER, Sanierung, $554 \mathrm{f}$. 
teiligungen verkauft werden. ${ }^{165}$ Weiter kann auch das Umlaufvermögen abgebaut werden, indem beispielsweise Lagerbestände reduziert und Debitorenfristen verkürzt werden. ${ }^{166}$

Zur Wiederherstellung der Ertragskraft kommen Rationalisierungs- sowie Kostensenkungsprogramme in Frage, welche etwa die Entlassung von Mitarbeitenden oder die Senkung von Produktionskosten umfassen können. ${ }^{167}$ Zur mittelfristigen Wiederherstellung der Rentabilität ist zudem wichtig, dass das Geschäftsmodell überprüft und allenfalls den Marktverhältnissen und Ressourcen angepasst wird. ${ }^{168}$

\section{Aufwertung von Grundstücken und Beteiligungen im Besonderen}

Wie bereits angetönt, stellt die Aufwertung von Grundstücken oder Beteiligungen eine bilanzmässige Sanierungsmassnahme dar. Im geltenden Aktienrecht ist diese Massnahme in Art. 670 sowie 671b OR geregelt. Art. 670 OR erlaubt es Unternehmen, im Falle eines Kapitalverlusts, Grundstücke und Beteiligungen $\mathrm{zu}$ ihrem wirklichen Wert $\mathrm{zu}$ bewerten (anstatt wie sonst $\mathrm{zu}$ Anschaffungs- oder Herstellungskosten) ${ }_{i}^{169}$ m.a.W. werden stille Reserven aufgelöst. ${ }^{170}$ In der Bilanz zeigt sich die Aufwertung durch ein grösseres Anlagevermögen auf der Aktivseite und durch die Bildung einer Aufwertungsreserve als Teil der gesetzlichen Reserve auf der Passivseite. ${ }^{171}$

Aufgrund des Bezugs zur Sanierung im OR wird die Bestimmung zur Aufwertung von Grundstücken und Beteiligungen neu als nArt. 725c OR in die aktienrechtlichen Sanierungsbestimmungen eingegliedert. ${ }^{172}$ Der bestehende Regelungsinhalt wird im neuen Aktienrecht grundsätzlich beibehalten. Neu wird nur explizit festgehalten, dass diese Bilanzierungsmassnahme nicht nur im Falle eines Kapitalverlusts, sondern auch bei einer Überschuldung zum Tragen kommt. ${ }^{173}$






\section{Würdigung}

Abschliessend möchten wir im Sinne einer Würdigung auf unsere eingangs gestellte Frage zurückkommen, ob das Sanierungsrecht im neuen Aktienrecht sein Hauptziel zu erreichen vermag.

Wie erwähnt, sollte das neue Sanierungsrecht im OR in erster Linie der Neugestaltung der „Frühwarnsysteme“ dienen: „Das Bewusstsein des Verwaltungsrates bezüglich Liquidität und Kapitaldeckung sollte geschärft werden, indem sowohl seine Handlungsspielräume erweitert und seine Pflichten konkretisiert werden ${ }^{\text {“174 }}$.

In Anbetracht dieses Hauptziels wird man diese Aktienrechtsreform in Bezug auf das Sanierungsrecht nicht als Totalrevision bezeichnen können. Sie vollzieht aber in gewissen Punkten immerhin eine erwünschte Aktualisierung und Kodifizierung der bestehenden Praxis. ${ }^{175}$ Alle Rechtsunsicherheiten werden durch die Revision aber nicht ausgeräumt, womit das Haftungsrisiko der Organe auch unter dem neuen Sanierungsrecht bestehen bleiben dürfte. ${ }^{176}$

Die unter dem neuen Tatbestand der drohenden Zahlungsunfähigkeit explizit vorgesehene Pflicht des Verwaltungsrats, die Zahlungsfähigkeit der Gesellschaft zu überwachen, dürfte dem entsprechen, was der Verwaltungsrat schon unter geltendem Recht tun muss, nachdem er im Rahmen der Finanzplanung die Liquiditätsplanung zu erstellen und die Liquiditätssituation zu überwachen hat. Möglicherweise wird durch die explizite Verankerung dieser Pflicht das Bewusstsein des Verwaltungsrats zur Liquiditätssicherung gestärkt, was zu wünschen wäre. Durch die systematische Integration dieser Aufgabe im Sanierungsrecht könnte aber auch der falsche Eindruck entstehen, dass die Liquiditätsplanung und -sicherung nur in der Krise der Gesellschaft vorzunehmen ist. Dies ist jedoch nicht der Fall, da die Überwachung der Liquiditätssituation eine dauernde Aufgabe des Verwaltungsrats ist. ${ }^{177}$

Das bestehende Problem, dass die meisten Verfahren im Konkurs enden werden und damit eben gerade keine Sanierung erfolgt, dürfte u.E. auch unter den neuen Bestimmungen des Sanierungsrechts bestehen bleiben. Für eine diesbezügliche grundlegende Änderung dürften einerseits die im revidierten Sanierungsrecht verankerten Bestimmungen zu wenig griffig sein und andererseits dürfte die Pflicht des Verwaltungsrats zur Ergreifung von Massnah-

\footnotetext{
$174 \mathrm{BBl} 2017,462 \mathrm{f}$.

175 Ähnlich auch FORSTMOSER/KÜCHLER, 28.

176 Glanzmann, Sanierung, 480.

177 GlanzmanN, Sanierung, 477.
} 
men auch unter dem neuen Recht oft zu spät einsetzen, um eine effektive Sanierung zu ermöglichen. Ergreift der Verwaltungsrat wie gesetzlich gefordert erst Massnahmen zur Sicherstellung der Zahlungsfähigkeit der Gesellschaft, wenn diese zahlungsunfähig zu werden droht, besteht meist bereits ein Zustand, in dem die Einstellung der Geschäftstätigkeit der Gesellschaft wegen Illiquidität voraussichtlich nicht mehr abwendbar ist. Aus diesem Grund wäre es u.E. sinnvoll, wenn der Warnruf des Gesetzes an den Verwaltungsrat wesentlich früher ansetzen würde, z.B. beim Eintritt von Zahlungsschwierigkeiten. ${ }^{178}$ In diesem Zeitpunkt könnte eine effektive Sanierung der Gesellschaft vielleicht noch gelingen ${ }^{179}$ Solange die Pflicht des Verwaltungsrats zur Ergreifung von Massnahmen indessen explizit erst an das Vorliegen einer drohenden Zahlungsunfähigkeit oder eines Kapitalverlusts anknüpft, wird sich wohl nicht viel daran ändern, dass die meisten Verfahren im Konkurs enden werden. ${ }^{180}$

Ob die Revision des aktienrechtlichen Sanierungsrechts ihr Hauptziel erreicht, ist vor diesem Hintergrund u.E. fraglich. Wir sind gespannt, wie die Praxis diese Frage beantworten wird.

\section{Literaturverzeichnis}

Basler Kommentar, Obligationenrecht II (Art. 530-964 OR), Hrsg: Honsell Heinrich/Vogt Nedim Peter/Watter Rolf, 5. A., Basel 2016 (zit. BSK OR II-BEARBEITER, Art. XX, N YY).

Basler Kommentar, Bundesgesetz über Schuldbetreibung und Konkurs II, Hrsg: Staehelin Adrian/Bauer Thomas/Staehelin Daniel, 2. A., Basel 2010 (zit. BSK SchKG II-BEARBEITER, Art. XX, N YY).

BAUER THOMAS, Nachhaltige Unternehmenssanierung, Ein Diskussionsbeitrag zur Revision des Obligationenrechts, ST 2010, $455 \mathrm{ff}$.

BÖCKLI Peter, Schweizer Aktienrecht, 4. A., Zürich/Basel/Genf 2009 (zit. BöcKLI, Aktienrecht).

BÖCKLi Peter, Die unentziehbaren Kernkompetenzen des Verwaltungsrates, in: Druey Jean Nicolas/Forstmoser Peter (Hrsg.), Schriften zum neuen Aktienrecht, Zürich 1994, 28 ff. (zit. BÖCKLI, Kernkompetenzen).

Boemle MaX/Stolz CARSTEn, Unternehmensfinanzierung, Bd. 1, Grundlagen und Kapitalbeschaffung, 14. A., Zürich 2010.

BÜHLER CHRISTOPH B., Sanierung nach Aktienrecht de lege lata et ferenda - Lösungsansätze zur Verbesserung der aktienrechtlichen Rahmenbedingungen der aussergerichtlichen Sanierung von Aktiengesellschaften, Zürich/Basel/Genf 2010 (zit. BüHLER, Sanierung). 
BÜHLER CHRISTOPH B., Aussergerichtliche Sanierung der Aktiengesellschaft, Lösungsansätze zur Verbesserung der aktienrechtlichen Rahmenbedingungen der Sanierung de lege ferenda, ST 2010, 444 ff. (zit. BÜHLER, Aktiengesellschaft).

Buser DAVID, Kommentar zu Art. 53/54 SIX Listing Rules, in: Baker \& McKenzie (Hrsg.), The SIX Swiss Exchange Listing Rules, Bern 2014.

Druey Jean Nicolas/Druey Just Eva/Glanzmann LuKas, Gesellschafts- und Handelsrecht, 11. A., Zürich/Basel/Genf 2015.

DUBACH AlEXANDER, Handlungsalternativen des Verwaltungsrates bei der Überschuldung der AG - Aussergerichtliche und gerichtliche Alternativen zum Konkurs, ST 1997, 53 ff.

Forstmoser PETER, Der Richter als Krisenmanager?, Überlegungen zu Art. 725 f. OR, in: Forstmoser Peter/Honsell Heinrich/Wiegand Wolfgang (Hrsg.), Richterliche Rechtsfortbildung in Theorie und Praxis - Methodenlehre und Privatrecht, Zivilprozess- und Wettbewerbsrecht, Festschrift für Hans Peter Walter, Bern 2005, 263 ff. (zit. FoRSTMOSER, Krisenmanager).

Forstmoser PeTER, Die aktienrechtliche Verantwortung, 2. A., Zürich 1987 (zit. ForsTMOSER, Verantwortung).

ForSTMOSER PETER/KÜCHLER MARCEL, Schweizerische Aktienrechtsreform: Die Schlussrunde ist eingeläutet!, Jusletter, 10. Februar 2020.

Forstmoser Peter/Meier-Hayoz Arthur/Nobel Peter, Schweizerisches Aktienrecht, Bern 1996.

GLANZMANN LUKAS, Konzept und Praxis der aktienrechtlichen Sanierung, SZW 2019, 465 ff. (zit. GLANZMANN, Sanierung).

GLANZMANN LUKAS, Ausgewählte Fragen im Zusammenhang mit Dividendenausschüttungen, in: Kunz Peter V./Arter Oliver/Jörg Florian S. (Hrsg.), Entwicklungen im Gesellschaftsrecht XII, Bern 2017, 83 ff. (zit. GLANZMANN, Dividendenausschüttungen).

GLANZMANN LUKAS, Drohende Zahlungsunfähigkeit, Kapitalverlust und Überschuldung, Das neue aktienrechtliche Sanierungsrecht, GesKR 2017, 387 ff. (zit. GLANZMANN, Zahlungsfähigkeit).

GLANZMAnn LuKaS, Das neue Sanierungsrecht, in: Kunz Peter V./Arter Oliver/Jörg Florian S. (Hrsg.), Entwicklungen im Gesellschaftsrecht X, Bern 2015, 225 ff. (zit. GlanzmanN, Sanierungsrecht).

GLANZMANN LUKAS, Haftungsrisiken der Leitungsorgane in der finanziellen Krise des Unternehmens, in: Kunz Peter V./Jörg Florian S./Arter Oliver (Hrsg.), Entwicklungen im Gesellschaftsrecht IX, Bern 2014, 247 ff. (zit. GLANZMANN, Haftungsrisiken).

GLANZMANn LuKas, Die Pflichten des Verwaltungsrates und der Geschäftsführung in finanziellen Krisensituationen, in: Roberto Vito (Hrsg.), Sanierung der AG, Ausgewählte Rechtsfragen für die Unternehmenspraxis, 2. A., Zürich 2003, 19 ff. (zit. GlanZManN, Pflichten).

GLANZMANN LUKAS/WOLF MARKUS, Haftung faktischer Organe für Schäden aus Konkursverschleppung, in: Sprecher Thomas (Hrsg.), Sanierung und Insolvenz von Unternehmen IX, Neue Entwicklungen, Zürich/Basel/Genf 2019, $21 \mathrm{ff}$. 
HACHMANN CHRISTIAN, Zahlungsunfähigkeit in der aktienrechtlichen Sanierung, in: Sprecher Thomas (Hrsg.), Sanierung und Insolvenz von Unternehmen IX, Neue Entwicklungen, Zürich/Basel/Genf 2019, 99 ff.

HANDSCHIN LUKAS, Die Pflichten und Verantwortlichkeit des Verwaltungsrates im Sanierungsfall, ZBJV 136/2000, $433 \mathrm{ff}$

KÄLIN OLIVER, Die Sanierung der Aktiengesellschaft, Ein Rechtshandbuch für Verwaltungsräte, Zürich/Basel/Genf 2016.

KeSSELBACH StEPHAN, Krise und Sanierung bei Aktiengesellschaften - insbesondere aus strafrechtlicher Sicht: unter besonderer Berücksichtigung des Art. 152 StGB, Diss. Zürich 2001, SSHW 204

KRNETA GEORG, Praxiskommentar Verwaltungsrat: Art. 707-726, 754 OR und Spezialgesetze: ein Handbuch für Verwaltungsräte, 2. A., Bern 2005.

MeIer-Hayoz ARTHur/Forstmoser Peter/Sethe Rolf, Schweizerisches Gesellschaftsrecht, 12. A., Bern 2018.

MÜLLER LUKAS, Das neue Sanierungsrecht aus empirischer Perspektive: Was sind die kritischen Erfolgsfaktoren einer Sanierung?, AJP 2014, $187 \mathrm{ff}$.

MÜlLER ROLAND/LipP LORENZ/PlÜSS ADRIAN, Der Verwaltungsrat, Ein Handbuch für Theorie und Praxis, 4. A., Zürich/Basel/Genf 2014.

SCHENKER URS, Möglichkeiten zur privatrechtlichen Sanierung von Aktiengesellschaften, SJZ 2009, $485 \mathrm{ff}$.

Schweizer Handbuch der Wirtschaftsprüfer, Bd. „Buchführung und Rechnungslegung“, Treuhandkammer (Hrsg.), Zürich 2014 (zit. Schweizer Handbuch).

Senn DorotheA, Die Haftung des Verwaltungsrats bei der Sanierung der AG, Diss. Basel 2001.

Simoniello Daniele, Die Überwachung der Liquidität durch den Verwaltungsrat, Geldflussrechnung und Liquiditätsplan unter Berücksichtigung der Art. 725 E-OR 2016, SJZ 2017, $541 \mathrm{ff}$.

SPRECHER THOMAS/SOMMER CHRISTA, Aktienrechtliche Sanierungsmassnahmen, in: Sprecher Thomas (Hrsg.), Sanierung und Insolvenz von Unternehmen VI, Zürich/Basel/Genf 2014, 35 ff. (zit. SPRECHER/SOMMER, Sanierungsmassnahmen).

Sprecher Thomas/SOMmer Christa, Sanierung nach Aktienrecht, Bestandsaufnahme und Handlungsbedarf, ST 2014, 551 ff. (zit. SPRECHER/SOMMER, Sanierung).

TEITLER-FEInBERG Evelyn/LipP LOREnZ, Beseitigung eines Kapitalverlusts durch Aufwertung von Aktiven?, Expert Focus 2018, $384 \mathrm{ff}$.

vON DER CRONe HaNs CASPAR, Aktienrecht, Bern 2014.

von der CRone Hans CASPar/Pfister LoÏC/SibBern ERIC, Revision des Aktienrechts: Sanierung, Begleitbericht, Mai 2006 (Vernehmlassungseingabe im Rahmen der Aktienrechtsund Rechnungslegungsrevision).

von der Crone Hans Caspar/Kopta-Stutz Bettina/Pfister LoÏc, Some Theses Concerning Modern Swiss Reorganization Law, ST 2005, 1028 ff.

Walz Hartmut/Gramlich Dieter, Investitions- und Finanzplanung, Eine Einführung in finanzwirtschaftliche Entscheidungen unter Sicherheit, 4. A., Heidelberg 1993. 
Zürcher Kommentar zum Schweizerischen Zivilgesetzbuch, Obligationenrecht, 5.Teil: Die Aktiengesellschaft, Der Verwaltungsrat, Art. 707-726 OR, Hrsg.: Gauch Peter/Schmid Jörg, 2. A., Zürich 1997 (zit. ZK OR-BEARBEITER, Art. XX, N YY). 



\title{
Sanierung und Einlagensicherung nach neuem Bankenrecht
}

\author{
Reto Schiltknecht*
}

\section{Inhalt}

I. $\quad$ Einleitung 158

II. Grundlagen 159

III. Sanierungsarten und -verfahren 161

1. Arten von Sanierungen 161

2. Verfahren 162

a) Voraussetzungen der Durchführung einer Sanierung 162

b) Genehmigung des Sanierungsplans 163

IV. Kernanliegen der Revision des Bankengesetzes 164

1. Kapitalmassnahmen 165

a) Hauptziele 165

b) Übersicht über die gesetzlichen Bestimmungen des Art. 30c E-BankG 165

2. Wertausgleich bei Kapitalmassnahmen 169

a) Hauptziele 169

b) Übersicht über die Konzeption des Wertausgleichs nach Art. 31c E-BankG 169

V. Beschwerdeverfahren 171

VI. Änderung bei Konkurszuständigkeit der FINMA 172

VII. Einlagensicherung 172

1. Vorgeschichte und Anlass zur Revision $\quad 172$

2. Zweck und Funktionsweise der Einlagensicherung 173

3. Zu den Reformbestrebungen im Einzelnen 174

a) Auszahlung 174

b) Vorbereitungsarbeiten der Banken 175

c) Exkurs: Vorbereitungsarbeiten und systemrelevante Banken $\quad 175$

d) Finanzierung 176

* Ergänzte Fassung des im Rahmen der 11. Tagung zur Sanierung und Insolvenz von Unternehmen vom 9. Juni 2020 gehaltenen Referates. Seither hat der Bundesrat die Botschaft zur Änderung des Bankengesetzes (Insolvenz, Einlagensicherung, Segregierung) am 19. Juni 2020 verabschiedet (siehe Medienmitteilung des Bundesrates vom 19. Juni 2020) (im Folgenden BBl 2020, 6359 ff.). Dieser Beitrag nimmt die Ausführungen der Botschaft auf. Der Autor vertritt ausschliesslich seine persönliche Meinung und bindet die Eidgenössische Finanzmarktaufsicht FINMA dadurch nicht. 
4. Bewertung der aktuellen Revision

VIII. Angrenzende Gebiete $\quad 177$

1. Segregierung 177

a) Begriff 177

b) Zweck und Inhalt der Segregierungspflicht 178

c) Datenübermittlung 178

2. Revision Pfandbriefgesetz 179

\begin{tabular}{lr} 
IX. Schlussbetrachtungen & 179 \\
\hline
\end{tabular}

\section{Einleitung}

Das Bankeninsolvenzrecht ${ }^{1}$ ist ein Sonderrecht für Banken und Wertpapierhäuser (früher Effektenhändler). ${ }^{2}$ Art. 28-32 Bankengesetz (BankG) regeln das Sanierungsverfahren und Art. 33-37g den Bankenkonkurs inklusive die privilegierten Einlagen. Die Bestimmungen zur Einlagensicherung finden sich in den Art. 37h-1 BankG. Der Sonderrechtsstatus bedeutet jedoch nicht, dass das Bundesgesetz über Schuldbetreibung und Konkurs ${ }^{3}$ keine Anwendung findet. Dieses ist anzuwenden, soweit dessen Anwendungsbereich im Bankengesetz nicht eingeschränkt wird. Zu diesem Zweck sieht Art. 25 Abs. 3 BankG vor, dass die Bestimmungen des Nachlassverfahrens des SchKG, des aktienrechtlichen Moratoriums (Art. 725 und Art. 725a OR) sowie die Pflicht zur Benachrichtigung des Richters (Art. 729b Abs. 2 OR) auf Bankinsolvenzen nicht anwendbar sind. Wichtige Ausführungsbestimmungen zur Bankensanierung, aber auch zum Bankenkonkurs befinden sich in der Bankeninsolvenzverordnung der Eidgenössischen Finanzmarktaufsicht FINMA. ${ }^{4}$

Das Sonderrecht wurde aufgrund der Erfahrungen aus dem Konkurs der Sparund Leihkasse Thun in den 90-iger Jahren des vergangenen Jahrhunderts geschaffen und trat per 1. Juli 2004 in Kraft. Der damaligen Botschaft zur Änderung des Bundesgesetzes über die Banken und Sparkassen vom 20. November $2002^{5}$ ist u.a. folgendes zu entnehmen:

1 Der Begriff Bankeninsolvenz umfasst sowohl die Bestimmungen des 11. Abschnittes: Massnahmen bei Insolvenzgefahr als auch des 12. Abschnittes: Konkursliquidation insolventer Banken (Bankenkonkurs) des Bankengesetzes.

2 Zum Begriff Wertpapierhaus vgl. Art. 41 FINIG.

3 Bundesgesetz über Schuldbetreibung und Konkurs vom 11. April 1889 (SchKG, SR 281.1).

4 Verordnung der Eidgenössischen Finanzmarktaufsicht über die Insolvenz von Banken und Wertpapierhäusern vom 30. August 2012 (Bankeninsolvenzverordnung-FINMA, BIV-FINMA, SR 952.05).

5 Botschaft des Bundesrates zur Änderung des Bundesgesetzes über die Banken und Sparkassen vom 20. November 2002, BBl 2002, 8060 ff. 
„Die hier vorgelegte Botschaft basiert auf einem Bericht von Experten aus Lehre, Praxis und Verwaltung. Sie sieht eine Änderung des Bankengesetzes vor, welche im Bereich der Sanierung und Liquidation von Banken die in zahlreichen Erlassen verstreuten Bestimmungen im Bankengesetz zusammenfasst und auf das Wesentliche konzentriert. Das Zusammenspiel von Aufsichts-, Sanierungs- und Liquidationsrecht wird optimiert. Die Eidgenössische Bankenkommission soll für die Leitung der Verfahren allein zuständig sein, also auch bei der Sanierung und im Bankenkonkurs."

In Bezug auf die Einlagensicherung ist auf die folgende Stelle in der Botschaft des Jahres 2002 hinzuweisen, woraus ersichtlich ist, dass eine obligatorische Einlagensicherung in der Schweiz erst 2004 eingeführt wurde:

„Schliesslich werden die privilegierten Einlagen durch eine nunmehr obligatorische, sich weitgehend auf eine Selbstregulierung der Banken stützende Einlagensicherung geschützt, welche von der Bankenkommission zu genehmigen ist."

Da die meisten Banken als Aktiengesellschaften organisiert sind, passt die Darstellung der in der Bankensanierung und Einlagensicherung vorgesehenen Neuerungen gut zum Tagungsthema „Die aktienrechtliche Sanierung“.

\section{Grundlagen}

Das Bankensanierungsrecht ${ }^{6}$ ist auf sämtliche Banken anwendbar. Im Rahmen dieses Beitrags werden wir uns aber überwiegend mit den systemrelevanten Instituten befassen. ${ }^{7}$ In der Schweiz sind folgende Banken bzw. Finanzgruppen von der Schweizerischen Nationalbank (SNB) als systemrelevant bezeichnet worden: UBS, Credit Suisse, die Raiffeisen-Gruppe, die Zürcher Kantonalbank sowie die PostFinance.

Ein ungeordneter Konkurs insbesondere einer systemrelevanten Bank könnte die Stabilität des schweizerischen Finanzsystems ernsthaft gefährden und im Falle der global tätigen Finanzgruppen UBS und Credit Suisse - sogar ein Risiko für die globale Finanzstabilität darstellen. Der Grund für die Exponiertheit dieser Institute liegt in ihrer besonderen volkswirtschaftlichen Rolle bei der Kredit- und Liquiditätsversorgung und der Erbringung von Dienstleistungen im Zahlungsverkehr begründet. Bei den vorgenannten global tätigen Finanzgruppen kommt ihre starke Vernetzung mit den grössten und wichtigs-

Art. 28-32 BankG.

7 Zum Begriff der systemrelevanten Bank vgl. Art. 7/8 BankG. 
ten Finanzinstituten weltweit hinzu. ${ }^{8}$ Das Bankengesetz sieht daher besondere Anforderungen für diese Bankenpopulation vor mit dem Ziel der Verhinderung eines (ungeordneten) Konkurses und der Schaffung von Voraussetzungen, damit eine Sanierung erfolgreich durchgeführt werden könnte. ${ }^{9}$

Zum besseren Verständnis der nachfolgenden Ausführungen wird eine beschränkte Auswahl der besonderen Anforderungen dargestellt, denen die systemrelevanten Banken und Finanzgruppen unterworfen sind.

- Damit die Sanierung eine realistische Option ist, müssen die betroffenen Institute in „guten Zeiten“ Vorbereitungen treffen. Allenfalls müssen (Organisations-)Strukturen ex-ante so angepasst werden, damit in der Kürze der Zeit (die Annahme ist, dass die Sanierung über ein einziges Wochenende erfolgen müsste) angesichts der Grössenverhältnisse und der Komplexität eine Sanierung überhaupt eine Chance hat, erfolgreich durchgeführt werden zu können. ${ }^{10}$ Damit systemrelevante Funktionen wie der ununterbrochene Zugang zur Liquidität oder die Aufrechterhaltung der Kreditversorgung in einer Krise gewährleistet werden könnten, haben die systemrelevanten Banken sog. Notfallpläne zu erstellen und der FINMA zur Genehmigung einzureichen.

- Zur Erhöhung der Krisenresistenz müssen sie besondere Eigenmittel- und Liquiditätsvorschriften erfüllen.

- Kommt es trotz der erhöhten Kapital- und Liquiditätspolster zu einer Situation, die eine Sanierung erforderlich macht, stehen in Form von „Krisenkapital" Mittel zur Rekapitalisierung der eingetretenen Verluste zur Verfügung. Die systemrelevanten Banken sind verpflichtet, diese Mittel in Form von sog. Bail-in-Anleihen (Bonds) auszugeben. Wenn Verluste eintreten, verfügt die FINMA über die gesetzliche Kompetenz (Art. 31 BankG), die Anleihen in Eigenkapital zu wandeln oder herabzuschreiben. Auf diese Weise tragen diejenigen Gläubiger die Verluste, die die Anleihen erworben haben, sich der oben dargestellten Konsequenzen bewusst waren und für die Übernahme des Verlustrisikos speziell entschädigt werden. Man spricht in diesem Zusammenhang von einem Bail-in im Gegensatz zu einem Bailout, in welchem der Staat die Verluste trägt und die Bank mit Steuergeldern vor dem Untergang bewahrt.

8 Vgl. die Medienmitteilung des Basler Ausschusses für Bankenaufsicht vom 22. November 2019, in welcher die aktuell systemrelevanten Banken und Finanzgruppen publiziert worden sind.

9 Vgl. zu den besonderen Anforderungen Art. 9 ff. BankG, Art. 60-66 BankV, Art. 124 ff. ERV sowie Art. 19 ff. LiqV.

10 Ein Beispiel für die Strukturänderungen sind die Gründung von eigenen Schweizer Banken bei UBS und Credit Suisse im Jahr 2015 (UBS) und 2016 (Credit Suisse). 
- Schliesslich ist nochmals darauf hinzuweisen, dass das Bankensanierungsrecht von seiner Konzeption her nicht nur auf systemrelevante, sondern auf sämtliche dem Bankgesetz unterstellten Institute anwendbar ist. Da aber nur die erstgenannte Gruppe von Banken den besonderen Anforderungen unterstellt ist, werden die Chancen, eine Sanierung erfolgreich durchführen zu können, bei den übrigen Banken erheblich vermindert sein. Dies liegt im Wesen der Bankensanierung begründet, die darauf abzielt, eine Bank rasch zu restrukturieren und zu rekapitalisieren, um sie dann, in den meisten Fällen in veränderter Struktur, mit neuen Organen und mit angepasstem Geschäftsmodell, fortzuführen. Gelingen kann eine solche Sanierung nach der hier vertretenen Auffassung nur dann, wenn, wie dies bei den systemrelevanten Banken der Fall ist, eine Pflicht zur umfassenden Vorbereitung mittels Notfallplänen usw. gesetzlich statuiert ist. Entsprechende Pflichten bestehen für weitere Banken derzeit nicht.

\section{Sanierungsarten und-verfahren}

\section{Arten von Sanierungen}

Gerät ein Unternehmen in Schieflage, gibt es eine Vielzahl von Möglichkeiten, um aus dieser misslichen Lage herauszufinden. Die Situation stellt sich bei Banken nicht anders dar als bei anderen Unternehmen mit dem Unterschied, dass die Aufsichts- bzw. die Resolution-Behörde ein gewichtiges Wort mitzureden hat. Die FINMA ist sowohl für die Aufsicht wie auch für die Insolvenz (Sanierung und Abwicklung) der von ihr beaufsichtigten Finanzmarktteilnehmer zuständig und vereinigt sämtliche Zuständigkeiten und Kompetenzen bei sich. Neben den Banken betrifft dies vor allem auch Versicherungsunternehmen und Asset Manager.

Im Folgenden sollen exemplarisch kurz einige mögliche Arten von Sanierungen aufgezählt werden (ohne Anspruch auf Vollständigkeit). Es gilt zunächst zwischen freiwilliger und unfreiwilliger Sanierung zu unterscheiden. Bei ersterer lässt sich eine Sanierung des Unternehmens mit seinen Gläubigern ohne die Involvierung der dafür zuständigen Behörde oder der Gerichte dadurch finden, dass die Gläubiger auf Forderungen ganz oder teilweise verzichten, frische Mittel (z.B. durch neue Investoren) in die Unternehmung fliessen oder diese veräussert werden kann, sei dies als Gesamtes oder den intergralen oder partiellen Verkauf von Aktiven und ev. auch Passiven. Bei letzter kommt eine freiwillige Sanierung nicht oder nicht rechtzeitig zustande. Die Gründe dafür können mannigfaltig sein und sind hier nicht darzustellen. Jedenfalls stellt sich 
die Situation so dar, dass die FINMA als Resolution-Behörde mittels staatlichen Zwangsmassnahmen eingreift. Sie hat die Möglichkeit, einen Finanzmarktteilnehmer zu sanieren oder über diesen den Konkurs zu verhängen. Im Rahmen dieses Beitrags befassen wir uns ausschliesslich mit den Banken und deren Sanierung, so dass nachfolgend nur davon die Rede sein soll.

Grundsätzlich bietet die Sanierung, wenn sie zulässig ist (siehe dazu Ziff. 2 hiernach) und mit Aussicht auf Erfolg durchgeführt werden kann, Vorteile gegenüber dem Konkurs. Bei den systemrelevanten Banken, die hier im Fokus stehen, kann durch die Sanierung mit grosser Wahrscheinlichkeit das Finanzsystem geschützt werden (Schutz der Systemstabilität). ${ }^{11}$ Sodann können die systemrelevanten Funktionen in der Schweiz aufrecht erhalten werden ${ }^{12}$ und das Risiko einer Ansteckungsgefahr, d.h. die Verbreitung der Krise auf weitere Banken aufgrund ihres hohen Grades an Vernetzung und gegenseitiger Abhängigkeit (gleichsam einem Domino-Effekt), kann reduziert (ev. sogar minimiert) werden. Schliesslich führt die auf Fortführung ausgerichtete Bankensanierung zu einem Werterhalt für die Gläubiger, weil beispielsweise keine Verkäufe unter hohem zeitlichem Druck (sog. fire sales) vorgenommen werden müssen. $^{13}$

\section{Verfahren}

\section{a) Voraussetzungen der Durchführung einer Sanierung}

Es muss begründete Aussicht auf die Sanierung der Bank oder einzelner Bankdienstleistungen bestehen (Art. 28 Abs. 1 BankG). Zum Zeitpunkt des Entscheides der FINMA muss hinreichend glaubhaft sein, dass die Gläubiger in der Sanierung voraussichtlich wirtschaftlich nicht schlechter gestellt sind als im Konkurs (Art. 30c Abs. 1 Bst. b E-BankG; siehe auch die Ausführungen unter b) hiernach zur Frage, ob das Verfahren zeitlich und sachlich durchführbar ist. ${ }^{14}$ Die Einhaltung des „No-Creditor-Worse-off-Test" dürfte in den aller-

11 Im Zentrum steht die Systemstabilität in der Schweiz. Bei den stark international tätigen Schweizer Grossbanken kann die Sanierung auch einen Beitrag zum Schutz der Finanzstabiliät in Drittstaaten leisten.

12 Zum Begriff der systemrelevanten Funktionen vgl. II.

13 Im Gegensatz zur Bankensanierung steht bei der nachlassvertraglichen Sanierung nach SchKG oft die Vermögensabtretung im Vordergrund und nicht die Fortführung des Betriebs.

14 Art. 31 Abs. 1 Bst. b BankG sieht noch die Besserstellung der Gläubiger gegenüber dem Konkurs vor. Die Neuerung der Nicht-Schlechterstellung entspricht den Key Attributes des FSB und ist zweckmässig. Vgl. BBl 2020, 6392. 
meisten Fällen zu überwinden sein, da die Sanierungswerte auf der Fortführung des Instituts berechnet werden und höher sind als die Erlöse, die im Konkurs erzielt werden können. ${ }^{15}$

\section{b) Genehmigung des Sanierungsplans}

Das Ziel der Sanierung besteht darin, dass die sanierte Bank die Bewilligungsvoraussetzungen und die übrigen gesetzlichen Vorschriften wieder dauerhaft einhält (Art. 29 BankG). Die getroffenen Massnahmen sind im Sanierungsplan enthalten, welcher von der FINMA genehmigt werden muss und in den Grundzügen öffentlich bekannt zu machen ist (Art. $31 \mathrm{E}-\mathrm{BankG}$ ). Während der Sanierungsplan immer öffentlich bekannt zu machen ist, sieht Art. 26 Abs. 2 EBankG neu vor, dass von der Publikation einer Schutzmassnahme Umgang genommen werden kann, wenn dadurch deren Zweck vereitelt würde. Dies könnte beispielsweise bei gesetzlichen Publikationspflichten aufgrund von Börsenvorschriften der Fall sein. Die Bankorgane wären diesfalls zur Offenlegung verpflichtet und werden davon aus haftungsrechtlichen Gründen nur Abstand nehmen, wenn die FINMA die Nicht-Publikation anordnet.

Die FINMA kann neu den Sanierungsplan einer systemrelevanten Bank auch genehmigen, wenn die Voraussetzungen der Nicht-Schlechterstellung der Gläubiger gegenüber dem Konkurs nicht erfüllt sind, sofern diese angemessen entschädigt werden (Art. 31 Abs. 3 E-BankG). Wird der sog. „No-CreditorWorse-off-Test" nicht beststanden, haben die betroffenen Gläubiger dies hinzunehmen, sofern sie eine angemessene Entschädigung erhalten. Diese kann z.B. in Beteiligungspapieren, Optionen oder Besserungsscheinen bestehen. ${ }^{16}$ Mit dieser Regelung hat der Gesetzgeber dem Systemschutz den Vorrang gegenüber den Gläubigerinteressen gegeben, allerdings unter der (gerichtlich überprüfbaren) Auflage der angemessenen Entschädigung für die Schlechterstellung gegenüber dem Konkurs.

Die Elemente, welche vorliegen müssen, damit die FINMA den Sanierungsplan genehmigen darf, sind in Art. 30c E-BankG festgehalten. Die ursprünglich in Art. 31 Abs. 1 BankG statuierten Genehmigungsvoraussetzungen befinden sich nunmehr in Art. 30c Abs. 1 E-BankG. Materiell neu ist einzig, dass die Passiven bei der Bewertung des Sanierungsbedarfs nun ausdrücklich qua Gesetz mit zu berücksichtigen sind. Im derzeit noch geltenden Art. 31 Abs. 1 BankG sind diese nicht ausdrücklich erwähnt, sondern nur die Aktiven. Klar war aber

$15 \quad$ BBl 2020, 6392.

16 Vgl. die Aufzählung in BBl 2020, 6394. Die Aufzählung ist nicht abschliessend. Es sind auch andere Formen der Entschädigung denkbar, wie z.B. eine Entschädigung in bar, sofern der Zweck der Sanierung dadurch nicht gefährdet wird. 
immer schon, dass die Passiven Bestandteil der Bewertung sein müssen. Das Vorsichtsprinzip ist grundsätzlich in Anschlag zu bringen, d.h. bei der Bewertung der Aktiven und Passiven sowie bei der Ermittlung des Sanierungsbedarfs.

Da die Planelemente, welche die FINMA im Rahmen der Genehmigung des Sanierungsplans zu berücksichtigen hat (Art. 30c Abs. 2 E-BankG), praktisch wortwörtlich von Art. 44 BIV-FINMA übernommen wurden, wird davon Abstand genommen, diese darzustellen und zu erläutern. Wichtig ist $\mathrm{zu}$ erwähnen, dass die Aufzählung in Absatz 2 nicht abschliessend ist und von der FINMA im konkreten Anwendungsfall weitere, für die Plangenehmigung wichtige Kriterien herangezogen werden können. Die Anhebung des BIV-Artikels auf Gesetzesstufe ist angesichts der Bedeutung des Sanierungsplans zu begrüssen. Selbstredend sind im Rahmen der Plangenehmigung die Einhaltung der Bewilligungsvoraussetzungen, die neue Kapitalstruktur und das Geschäftsmodell sowie die wirtschaftliche Lage der restrukturierten Bank umfassend darzulegen. Eine hervorragende Bedeutung kommt dem Vorsichtsprinzip im Zusammenhang mit den Ausführungen zur wirtschaftlichen Lage zu. Die Lage darf angesichts möglicher zukünftiger Verluste sowie genereller Geschäftsrisiken und weiterer Unwägbarkeiten (z.B. verbunden mit der geographischen Verteilung des Geschäftes) nicht (zu) rosig dargestellt werden.

\section{Kernanliegen der Revision des Bankengesetzes}

Folgende Anliegen stellen die Hauptpfeiler der Revision dar:

- Die Stärkung der Rechtsgrundlagen, um den Bail-in möglichst rechtssicher durchführen zu können. Zu diesem Zweck dient die Anhebung div. bisher in der BIV-FINMA vorgesehener Bestimmungen auf die Stufe formelles Gesetz;

- Eine noch verbesserte Abstimmung mit internationalen Standards, namentlich den Key Attributes of Effective Resolution Regimes for Financial Institutions (Key Attributes) des FSB;

- Die Beseitigung bestehender Unklarheiten;

- Verbesserungen im Bereich des Beschwerdeverfahrens;

- Weiterentwicklung des Einlagensicherungssystems; und

- Schliessung von Lücken im Bereich der Segregierung von Wertschriften.

Die folgenden Kapitel enthalten im Wesentlichen Ausführungen zu den auf der Ebene der Kernanliegen vorgesehenen gesetzlichen Änderungen. Daneben wird auf weitere Änderungen, auch in anderen Gesetzen als dem Bankengesetz, hingewiesen. Diese werden in der gebotenen Kürze erläutert. 


\section{Kapitalmassnahmen}

a) Hauptziele

Der neue Art. 30b E-BankG ist zentral für die Revision. Er regelt nunmehr den Bail-in umfassend auf Stufe des Bankengesetzes. Hauptziele der Anpassungen sind:

- Stärkung der Legitimation des Bail-in;

- Verbesserung der Rechtssicherheit/Durchsetzbarkeit; und

- Einhaltung der internationalen Standards, konkret der FSB Key Attributes.

\section{b) Übersicht über die gesetzlichen Bestimmungen des Art. 30c E-BankG}

Absatz 1 befasst sich mit den Grundvoraussetzung des Bail-in und ist dem bisherigen Art. 31 Abs. 3 BankG nachgebildet. Danach kann der Sanierungsplan die Reduktion des bisherigen Eigenkapitals und die Schaffung von neuem Eigenkapital, die Wandlung von Fremd- in Eigenkapital sowie die Reduktion von Forderungen vorsehen. Neu ist der Bail-in aber nicht mehr eine subsidiäre Massnahme, die nur dann zum Zug kommen darf, wenn die drohende Insolvenz der Bank nicht auf andere Weise beseitigt werden kann. Die FINMA muss im konkreten Fall eine Abwägung zwischen verschiedenen Interessen vornehmen. Die Botschaft spricht von Gläubigerschutz, Systemstabilität und Eignerinteressen. ${ }^{17}$ Dass der Bail-in nicht mehr die „ultima ratio“ darstellt ist zu begrüssen und gewährt der FINMA die nötige Flexibilität, der konkreten Situation angepasste Massnahmen anordnen zu können.

Absatz 2 stellt etwas klar, was im Rahmen des Bail-in ebenso zentral wie unumstritten sein sollte: nämlich, dass im Rahmen eines Bail-in keine Bezugsrechte an bisherige Eigner zugeteilt werden. Gegeben die Konzeption des schweizerischen Bail-in-Ansatzes, wonach ein Bail-in erst angeordnet werden kann, sofern sämtliche Eigner mit all ihren Rechten, wozu auch Bezugsrechte gehören, „weggeputzt” (wiped-out) worden sind, ist die Klarstellung zweckmässig und zu begrüssen.

Absatz 3 regelt, welche Forderungen von einem Bail-in ausgenommen sind. Er entspricht im Wesentlichen Art. 49 BIV-FINMA, so dass nur auf Neuerungen einzugehen ist. Gemäss Bst. d sind Forderungen vom Bail-in auszunehmen, die (i) während der Dauer von Schutzmassnahmen oder (ii) während des Sanierungsverfahrens mit Zustimmung der FINMA eingegangen wurden. Wer in

$17 \quad$ BBl 2020, 6386. 
einer kritischen Lage der Bank Leistungen erbringt, wird dies nur tun, wenn er vor einem Bail-in gefeit ist. Um die Funktionsfähigkeit des betroffenen Instituts zu gewährleisten, ist die Ausnahmeregelung wichtig. Absatz 4, wonach Forderungen aus Warenlieferungen und Dienstleistungen vom Bail-in ausgenommen werden können, wenn dies für die Weiterführung der Bank erforderlich ist, ist im selben Kontext zu sehen. Die Botschaft stellt zutreffenderweise klar, dass eine enge Auslegung geboten ist. ${ }^{18}$ Kriterien für die Gewährung der Ausnahme sind (i) die Erforderlichkeit der Leistung für die Weiteführung der Bank und (ii) das Fehlen der Substituierbarkeit. ${ }^{19}$

Absatz 5 sieht in Anlehnung an Art. 48 BIV-FINMA vor, dass die Wandlung bzw. Herabschreibung der verlusttragenden Instrumente gemäss Art. 11 BankG (sog. Contingent Convertibles oder CoCos) in einem ersten Schritt erfolgt sein muss, bevor - gleichsam als Vorbereitungsmassnahme für den Bail-in - das gesamte Gesellschaftskapital herabgesetzt wird. Die vorgängig in Eigenkapital gewandelten CoCos sind ebenfalls davon betroffen. Faktisch handelt es sich bei den „Wandlern“ um Instrumente mit der Wirkung der Herabschreibung. Da diese Art von Instrumenten ökonomisch gesehen wenig sinnvoll ist, hat der Markt sie „ausrangiert".

Gemäss Absatz 6 soll der Bundesrat Schuldinstrumente bezeichnen können, die bereits vor einer vollständigen Herabsetzung des Gesellschaftskapitals verlusttragend sind, d.h. in Wert reduziert werden (Wandlung ausgeschlossen). Voraussetzungen dafür sind, dass (i) die Ausgabe der Instrumente durch eine systemrelevante Bank in der Rechtsform der öffentlich-rechtlichen Anstalt mit ausdrücklicher Staatsgarantie erfolgt (derzeit trifft dies ausschliesslich auf die Zürcher Kantonalbank [ZKB] zu) und (ii) in den Anleihensbedingungen eine nachträgliche Kompensation der Gläubiger vorgesehen ist (mit der vielsagenden Einschränkung „sofern und soweit der Kanton im Rahmen der Staatsgarantie an die Sanierung der Bank beiträgt"). Laut Botschaft $^{20}$ soll es dem Kanton verwehrt sein, Bail-in-Bonds auszugeben, weil eine Wandlung zu Eigentum die Bondholder zu Eignern machen würde, was das kantonale Recht - die Beteiligung Dritter an der Bank - verbietet. Mindestens theoretisch gäbe es aber die Möglichkeit, dass der Kanton Bail-inBonds ausgeben bzw. erwerben würde, womit er bei einer Wandlung Eigen-

BBl 2020, 6388.

BBl 2020, 6388 erwähnt, dass insbesondere Forderungen aus Derivateverträgen und Interbankgeschäften vom Bail-in nicht ausgenommen werden dürfen. Dies ist zutreffend und ergibt sich bereits aufgrund einer grammatikalischen Auslegung des Gesetzestextes.

BBl 2020, 6389. 
tümer der Bank bliebe. ${ }^{21}$ Die Botschaft erwähnt weiter, dass auch die Ausgabe von Write-off-Instrumenten nicht möglich sei, da ansonsten der Test, wonach die Sanierung für die Gläubiger jedenfalls nicht schlechter ausfällt als der Konkurs, nicht mehr bestanden werde. Auch diese Argumentation soll vorliegend nicht weiter vertieft werden, obwohl gewisse Fragezeichen anzubringen sind. ${ }^{22}$. Was hingegen der Erörterung bedarf ist die vorgeschlagene Regelung, wonach die Gläubiger der erwähnten Instrumente nur im Sinne einer „Möglichkeit" zu entschädigen sind, nämlich nur dann, wenn der Kanton sich entschliesst, an die Sanierung beizutragen und nur in diesem Umfang. Begründet wird dies damit, dass ansonsten, d.h. bei einer Verpflichtung der Gläubigerentschädigung aus der Staatsgarantie, die Instrumente als nachrangig zu gelten hätten, was wiederum die Entschädigung gemäss Kantonalbankengesetz ausschliesse (die Entschädigungspflicht entfällt für nachrangige Forderungen). Dieser Argumentation kann nicht gefolgt werden. Zunächst ist darauf hinzuweisen, dass die Investoren in die beschriebenen Instrumente wie eine Art Schutzschild bereit sind, Verluste zu tragen, bevor das Dotationskapital herangezogen wird. Sie sind also bereit, anstelle des Staates einzuspringen. Dass sie dafür entschädigt sein wollen, scheint offensichtlich. Die ZKB wird kaum darum herumkommen, wenn sie die Instrumente erfolgreich platzieren will. Sodann ist das Argument der Nachrangigkeit nicht stichhaltig. Die Instrumente können und sollten - im Gegensatz zu Bail-in-Bonds - nicht nachrangig ausgestaltet werden, aber selbst wenn sie nachrangig wären, könnte sich der Kanton vermutlich vertraglich zu einer Entschädigung verpflichten. Der Bundesrat wird die Instrumente in der Eigenmittelverordnung (ERV) näher zu definieren haben. Die obigen Ausführungen verstehen sich als konstruktiven Beitrag zu diesem noch zu führenden Diskurs.

Absatz 7 beinhaltet den sog. Wasserfall, bezeichnet also die Reihenfolge des Bail-in und ist selbstredend absolut zentral. Grundsätzlich kann auf Art. 48 BIV-FINMA verwiesen werden. Soweit jedoch Neuerungen vorgeschlagen werden, ist darauf einzugehen. Im Gegensatz zur Regelung in der Bankeninsolvenzverordnung der FINMA enthalten Gesetz und Botschaft wertvolle Prä-

21 Es soll an dieser Stelle nicht weiter darauf eingegangen werden, ob es andere Möglichkeiten der Ausgabe von Schuldinstrumenten gibt, z.B. hartes Kernkapital, AT1- oder Tier 2-Instrumente, um die Anforderungen an die für den Fall der drohenden Insolvenz auszugebenden Mittel (sog. gone-concern Komponente) zu erfüllen.

22 So erfolgt beispielsweise die Gegenüberstellung der zu sanierenden Bank auf Basis einer Bewertung zu Fortführungswerten, wohingegen die konkursite Bank zu Liquidationswerten bewertet wird. Allein daraus kann sich ergeben, dass der „no creditor worse off“ zugunsten der Sanierung erfüllt wird. 
zisierungen. ${ }^{23}$ Zunächst wird präzisiert, was alles unter nachrangigen Forderungen zu verstehen ist. Insbesondere handelt es sich um Tier 2-Kapital, ob regulatorisch anrechenbar oder nicht. Andere Formen nachrangiger Forderungen sind auch eingeschlossen, sofern es sich nicht um Bail-in-Bonds handelt. Für diese ist ein eigener Rang geschaffen worden. ${ }^{24}$ Sie folgen unmittelbar auf die nachrangigen Forderungen (Art. 30b Abs. 7 Bst. b E-BankG). Die Investoren in diese Instrumente haben im Bewusstsein, dass sie weit vorne in der Rangfolge stehen und daher die Wahrscheinlichkeit, dass sie eingebailt werden, hoch ist, investiert. Die Bail-in-Bonds sind das eigentliche Rückgrat der Konzeption der Sanier- und Abwickelbarkeit („Resolvability“) systemrelevanter Banken, indem die Investoren in Bail-in-Bonds das hierfür erforderliche Kapital zur Verfügung stellen. ${ }^{25}$ Schliesslich folgen danach und können, soweit erforderlich, ebenfalls eingebailt werden, sämtliche Drittklassforderungen mit Ausnahme der Einlagen. Diese unterliegen einem Bail-in erst in letzter Linie.

Absatz 8 beschlägt die Regelung der sog. Clean Holding. Darunter ist zu verstehen, dass die auf Stufe Holding ausgegebenen Bail-in-Bonds in der Rangfolge gemäss Art. 30b Abs. 7 Bst. c (und nicht nach Bst. b) behandelt werden ${ }^{26}$ soweit die übrigen gleichrangigen Forderungen 5 Prozent der gesamthaft ausstehenden Bail-in-Bonds nicht übersteigen. Bezüglich der innerhalb dieser Limite bestehenden Forderungen wird von einem Bail-in abgesehen. Die Holding muss demnach „sauber" sein und "sauber" ist sie, wenn sie weniger als $5 \%$ der besagten Forderungen auf der Bilanz hat. Falls sie nicht sauber ist, hat dies zur Konsequenz, dass sämtliche Bail-in-Bonds nach Massgabe von Art. 30b Abs. 7 Bst. b E-BankG behandelt werden. Laut Botschaft ${ }^{27}$ ist für die Feststellung des Einhaltens der 5\%-Grenze der Zeitpunkt der Genehmigung des Sanierungsplans massgebend. Das ergibt Sinn, da einzig dieser einheitliche Ermittlungszeitpunkt sicherstellt, dass ein Auseinanderklaffen von Bailin-Bonds in der Rangfolge zum Zeitpunkt des Bail-in (Bst. b vs. Bst. c) verhindert werden kann. Mit der neuen Regelung sollen vor allem die operativ tätigen Tochtergesellschaften der Holding vor einem Bail-in geschützt, und es

23

BBl 2020, $6389 \mathrm{f}$.

Vgl. Art. 126a ERV, worin die gesetzlichen Anforderungen an Bail-in-Bonds umfassend geregelt sind. Deren Anrechnung durch die FINMA als gone-concern Mittel darf nur erfolgen, wenn die dort statuierten Bedingungen eingehalten sind.

Die Summen sind beträchtlich (40-50 Mrd. Schweizer Franken je G-SIB).

Man spricht in diesem Zusammenhang von struktureller Subordination, da sich der Bail-in nur auf Stufe Holding auswirkt und die unterliegenden Bankeinheiten davon nicht betroffen sind. Vor allem die beiden Schweizer Grossbanken UBS und Credit Suisse haben ihre Bail-in Bonds strukturell nachrangig emittiert.

BBl 2020, 6391. 
sollen die internationalen Standards eingehalten werden, gemäss welchen die Gläubiger der übrigen Forderungen im Vergleich zu den Bail-in Bonds fair zu behandeln sind.

Schliesslich sieht Absatz 9 eine neue Regelung vor. Die FINMA soll die Kompetenz erhalten, die Mitwirkungsrechte der durch Wandlung von Bail-in-Bonds neu geschaffenen Eigentümer vorübergehend, aber vollständig zu suspendieren. Es geht in erster Linie um die Ausübung der Stimmrechte in einer Phase nach der Sanierung, in welcher die Gesellschaft Stabilität benötig, um möglichst rasch zu einem normalen Geschäftsgang zurückzufinden. Falls es sich zeigen sollte, dass z.B. aktivistische Aktionärsgruppen die Stabilität des Instituts gefährden, ermöglicht die neue explizite Kompetenz ein Einschreiten im Interesse der Gewährleistung der Fortführung der eingeleiteten Sanierung. Die Suspension von Stimm- oder anderen Mitwirkungsrechten kann längstens bis zum Zeitpunkt der Durchführung der konstituierenden Generalversammlung angesetzt werden.

\section{Wertausgleich bei Kapitalmassnahmen}

\section{a) Hauptziele}

Mit dem Wertausgleich als Folge von Kapitalmassnahmen soll folgendes bezweckt werden:

- Die Gläubiger sollen durch ein Bail-in nicht auf Kosten der Eigner bereichert werden;

- An der vollständigen Herabsetzung des Gesellschaftskapitals vor der Durchführung des Bail-in soll festgehalten werden;

- Es sollen Lösungen gefunden werden können, die auf den konkreten Einzelfall zugeschnitten sind.

\section{b) Übersicht über die Konzeption des Wertausgleichs nach Art. 31c E-BankG}

Gemäss Art. 31c Abs. 1 E-BankG kann der Sanierungsplan einen angemessenen Wertausgleich für die Eigner vorsehen. Diese Bestimmung ist neu, und es fragt sich daher, weshalb sie eingeführt werden soll. Der Ursprung des Bedürfnisses nach einem Wertausgleich liegt darin begründet, dass das schweizerische Recht das vollständige "Wegputzen“ des Gesellschaftskapitals verlangt, bevor ein Bail-in durchgeführt werden darf. Es kann nun Situationen geben, in welchen dieser zu einem Zeitpunkt durchgeführt werden muss, in welchem die Bank noch über hohe Eigenmittel verfügt. Eine solche Situation 
kann insbesondere bei einer schweren Liquiditätskrise gegeben sein. ${ }^{28}$ Diesfalls müssen unter Umständen Sanierungsmassnahmen zu einem frühen Zeitpunkt eingeleitet werden, obwohl der Wert der Bank noch relativ hoch ist. Entsprechend werden durch den Bail-in und die vollständige Herabsetzung des Gesellschaftskapitals diese Werte von den Eignern auf die Gläubiger übertragen, erhalten diese doch mehr als ihre ursprüngliche Forderung. Durch deren Wandlung in Aktien sollen sie jedoch nicht mehr erhalten, als es dem Nominalwert ihrer Forderung entspricht.

Um die oben beschriebene Umverteilung auszugleichen, bedarf es eines Ausgleichs zugunsten der Eigner. Wie ist nun dieser Wertausgleich vorzunehmen?

Dieser soll ggf. im Sanierungsplan festgelegt werden. Grundlage bildet Art. 30c Abs. 1 Bst. a E-BankG. Danach beruht der Sanierungsplan auf einer vorsichtigen Bewertung der Aktiven und Passiven der Bank und einer vorsichtigen Schätzung des Sanierungsbedarfs. Angesichts der in diesem Zeitpunkt voraussichtlich bestehenden Unsicherheiten, wird für gewisse Bilanzpositionen kein zuverlässiger Marktwert bestehen, so dass man auf Schätzungen auf der Grundlage der dannzumal vorliegenden Informationen angewiesen sein wird. ${ }^{29}$ Trotz dieser Schwierigkeiten ist der Zeitpunkt der Festlegung des Wertausgleichs mit Genehmigung des Sanierungsplanes der richtige, um die notwendige Rechtssicherheit (klare Verhältnisse) für die sanierte Bank zu schaffen.

Gemäss Art. 31c Abs. 2 E-BankG erfolgt der Wertausgleich namentlich durch Zuteilung von Aktien, anderen Beteiligungsrechten, Optionen oder Besserungsscheinen. Die Aufzählung ist richtigerweise nicht abschliessend, so dass - je nach Bedürfnis des Einzelfalls - auch weitere Formen des Wertausgleichs zulässig sind. Die Botschaft ${ }^{30}$ äussert sich einlässlich zur Funktionsweise und möglichen Ausgestaltung von Genussscheinen und Call-Optionen. Genussscheine (Art. 657 OR) sind das klassische Instrument, um Personen zu entschädigen, die mit der Gesellschaft durch frühere Kapitalbeteiligung oder als Aktionär verbunden waren. Ohne hier auf die Funktionsweise der Optionen einzugehen, ist darauf hinzuweisen, dass bei einem physischen Bezug der Aktien die Bank als Gegenpartei über die entsprechende Anzahl Aktien verfügen muss, um den Lieferanspruch erfüllen zu können. Die Aktien kann sie auch nachträglich, d.h. nach Genehmigung des Sanierungsplanes, noch beschaffen. 
Damit ist allgemein gesagt, dass der Sanierungsplan zwar den Wertausgleich und dessen Höhe bestimmt, dass aber die Art und Weise der Anspruchserfüllung nicht im Detail im Plan bereits vorgezeichnet sein muss.

\section{Beschwerdeverfahren}

Die bisher unübersichtlichen Bestimmungen zum Beschwerdeverfahren sollen neu im Abschnitt 12a Beschwerden in Verfahren nach dem elften und zwölften Abschnitt zusammengefasst werden. Es handelt sich um die 4 Artikel Art. $37 \mathrm{~g}^{\text {bis }}$ bis Art. $37 \mathrm{~g}^{\text {quinquies }}$ E-BankG. ${ }^{31}$ Art. $37 \mathrm{~g}^{\text {bis }}$ befasst sich mit der Beschwerde gegen die Genehmigung des Sanierungsplans, Art. $37 \mathrm{~g}^{\text {ter }}$ mit Beschwerden von Gläubigern und Eignern, Art. $37 \mathrm{~g}^{\text {quater }}$ mit den Fristen und schliesslich Art. 37gquinquies mit der aufschiebenden Wirkung. Es soll im Folgenden nicht darum gehen, die Beschwerdeordnung umfassend dazustellen, sondern auf ein paar Besonderheiten bzw. Neuerungen hinzuweisen.

Zunächst ist darauf hinzuweisen, dass die Bestimmungen auf Massnahmen bei Insolvenzgefahr (elfter Abschnitt) und auf den Bankenkonkurs (zwölfter Abschnitt) gleichermassen Anwendung finden. Sodann soll, wie bisher, auch bei Gutheissung einer Beschwerde gegen die Genehmigung des Sanierungsplans ausschliesslich eine Entschädigung zugesprochen werden können. Die Sanierung würde verunmöglicht, wenn gegen die im Sanierungsplan getroffenen Massnahmen im einzelnen oder insgesamt Beschwerde erhoben werden könnte. Der Sanierungsplan muss unmittelbar in Kraft gesetzt werden können, damit er seine Wirksamkeit entfalten kann. Der Entschädigungsmechanismus (Art. 37g ${ }^{\text {bis }}$ Abs. 2 E-BankG) folgt den gleichen Grundsätzen wie die Entschädigung infolge Wertausgleichs. ${ }^{32} \mathrm{Neu}$ ist im Bereich des Bankenkonkurses, dass die betroffenen Gläubiger und Eigner gegen die Genehmigung der Verteilungsliste und der Schlussrechnung Beschwerde führen können. Die Botschaft $^{33}$ führt dazu zutreffend aus:

„[...] Die Schlussrechnung und die Verteilungsliste werden vom Konkursliquidator im Bankenkonkursverfahren erstellt und der FINMA zur Genehmigung unterbreitet (vgl. Art. 36 Abs. 2 BIV-FINMA). Diese Regelung ist im Lichte des verfassungsrechtlichen Vorbehaltes des Gesetzes und der Rechtsweggarantie jedoch problematisch, wird doch durch die Zulassung von Massakosten [...] die künftige Dividende [...] unmittelbar gekürzt.“

\footnotetext{
31 Siehe auch die Ausführungen in BBl 2020, $6398 \mathrm{ff}$.

32 Vgl. IV.2.b) hiervor.

33 Vgl. BBl 2020, 6400.
} 
Die Beschwerdefrist beträgt nunmehr für die Beschwerde gegen die Genehmigung des Sanierungsplans und gegen Verwertungshandlungen 10 Tage und beginnt im ersteren Fall am Tag nach der öffentlichen Bekanntgabe der Grundzüge des Sanierungsplans zu laufen (Art. $37 \mathrm{~g}^{\text {quater }}$ E-BankG). Wichtig ist, dass nach Art. 37g quinquies E-BankG gegen folgende Anordnungen der FINMA keine aufschiebende Wirkung einer Beschwerde besteht und vom Richter auch nicht angeordnet werden kann:

- Anordnung von Schutzmassnahmen;

- Anordnung eines Sanierungsverfahrens;

- Genehmigung des Sanierungsplans; und

- Anordnung der Konkursliquidation.

\section{VI. Änderung bei Konkurszuständigkeit der FINMA}

Art. 173b Abs. 2 SchKG sieht neu vor, dass nicht der Konkurszuständigkeit der FINMA Schuldner unterstehen, die nicht über die erforderliche Bewilligung der FINMA verfügen. Diese Neuerung ist von grosser praktischer Relevanz. Die Klarstellung der Kompetenzen zwischen FINMA und kantonalen Konkursbehörden im Bereich der Tätigkeit unbewilligter Institute ist im Interesse der Rechtssicherheit und eines raschen Verfahrensablaufs zu begrüssen. Die bisherige FINMA-Praxis bei der Festlegung ihrer Zuständigkeit knüpfte an der bewilligungspflichtigen Tätigkeit an. Dies führe in der Vergangenheit zu aufwändigen Verfahren zur Klärung der Frage, ob eine ausgeübte Tätigkeit bewilligungspflichtig war oder nicht. ${ }^{34}$ Da nunmehr nur noch Bewilligungsträger in die Zuständigkeit der FINMA fallen, entfällt die Unsicherheit über die Zuständigkeit und wird eine klare Kompetenzzuweisung an die kantonalen Konkursbehörden für illegal im Finanzbereich tätige Unternehmen vorgenommen.

\section{Einlagensicherung}

\section{Vorgeschichte und Anlass zur Revision}

Während der Finanzkrise 2007/2008 wurde der Einlegerschutz mittels dringlichem Bundesgesetz verstärkt. Folgende Neuerungen wurden ins ordentliche Recht überführt: 
- Erhöhung des Konkursprivilegs von CHF 30'000 auf CHF 100'000;

- Pflicht zur sofortigen Auszahlung der privilegierten Einlagen aus den liquidien Mitteln der Bank;

- Deckung der privilegierten Einlagen durch in der Schweiz gelegene Aktiven im Umfang von $125 \%$;

- Privilegierung von Vorsorgeguthaben; und

- Erhöhung der Systemgrenze von CHF 4 auf CHF 6 Milliarden. ${ }^{35}$

Nachdem 2009 eine Vorlage zur Stärkung des Einlagensicherungssystems, die die Umstellung auf eine vollständige Vorfinanzierung mit einem Einlagensicherungsfonds vorschlug, klar scheiterte, herrschte einige Jahre Funkstille. Erst die vom Bundesrat eingesetzte Expertengruppe Brunetti zur Weiterentwicklung der Finanzmarkstrategie nahm - nebst anderem - das Thema Stärkung des Einlagensicherungssystems wieder auf und schlug gewisse Korrekturen vor. Im Zentrum standen folgende Vorschläge:

- Übergang zu einer echten Auszahlungsfrist ${ }^{36}$ mit einer verkürzten Auszahlungsfrist;

- Erhöhung der Systemgrenze; und

- zumindest eine teilweise Vorfinanzierung der Einlagensicherung.

Gestützt auf die Empfehlung der Expertengruppe Brunetti beschloss der Bundesrat 2017 im Interesse der Finanzstabilität, das Einlagensicherungssystem weiter zu stärken und beauftragte das Eidg. Finanzdepartement mit der Ausarbeitung einer Vorlage. ${ }^{37}$

\section{Zweck und Funktionsweise der Einlagensicherung}

Das Einlagensicherungssystem erfüllt im Wesentlichen zwei Zwecke. Zum einem soll es im Sinne des Funktionsschutzes einen Beitrag dazu leisten, dass bei Auftreten von Finanzkrisen oder auch nur singulären Bankkonkursen die Einleger von der Forderung der sofortigen Ausbezahlung ihrer Einlagen abgehalten werden können. Wenn dieses Verhalten massiert auftritt, spricht man von einen Bankensturm („bank run“), der die Finanzstabilität erheblich beinträchtigen kann. Der Einlegerschutz kann einen Beitrag zur Verhinderung eines Bankensturms leisten, wenn er bei den Anlegern Vertrauen schafft, dass

35 Systemgrenze bedeutet, dass die Verpflichtungen sämtlicher Banken, Beiträge an das Einlagensicherungssystem zu leisten, den erwähnten Betrag nicht übersteigen dürfen.

36 Das bisherige System sieht keine eigentliche zeitliche Vorgabe zur Auszahlung der Einlagen, sondern lediglich eine Pflicht des Trägers der Einlagensicherung vor, seine Leistungen innerhalb einer bestimmten Frist an den Konkursliquidator auszubezahlen.

37 Siehe auch den sehr guten Überblick in BBl 2020, 6372 ff. über die international bestehenden Grundsätze sowie zum Rechtsvergleich mit der EU und den USA. 
auch im Konkursfall (mind. in begrenztem Umfang) Zugriff auf die Einlagen besteht. Zum anderen soll die Möglichkeit der Einleger, auch im Konkurs der Bank rasch über einen bestimmten (ausreichenden) Betrag zur Deckung der laufenden Kosten der Lebenshaltung zu verfügen, den Gläubigerschutz stärken.

Im Weiteren ist das Dreisäulenprinzip des Schweizer Einlegerschutzes kurz darzulegen. Am Anfang steht das Konkursprivileg, d.h. die Kollokation der Einlagen und der Guthaben bei Vorsorge- und Freizügigkeitsstiftungen in der 2. Konkursklasse (Art. 37a Abs. 1 und 5 BankG). Als weiteres Element kommt die Sicherung der Auszahlung des Privilegs durch das Bankensystem dazu, welches sich auf die Einlagen bei schweizerischen Geschäftsstellen erstreckt (nicht aber auf ausländische Zweigniederlassungen). Schliesslich sind - wie bereits erwähnt - vorab die liquiden Aktiven zur Deckung der privilegierten Einlagen beizuziehen.

Das schweizerische Einlagensicherungssystem fusst auf Selbstregulierung. Sämtliche Banken und Effektenhändler in der Schweiz müssen sich zum Zweck der Einlagensicherung dem privatrechtlich organisierten Verein esisuisse anschliessen (Zwangsmitgliedschaft, die bei der Bewilligungserteilung zur Auflage gemacht wird). esisuisse zieht im Anwendungsfall die Beiträge bei ihren Mitgliedern ein und leistet im erforderlichen Umfang Zahlung an den Konkursliquidator.

\section{Zu den Reformbestrebungen im Einzelnen}

\section{a) Auszahlung}

Nach Art. 37h Abs. 3 Bst. a E-BankG hat esisuisse seit Mitteilung der FINMA über die Anordnung der Konkursliquidation die gesicherten Einlagen innerhalb von 7 Tagen an den Konkursliquidator auszubezahlen. Die Frist wurde verkürzt; heute beträgt sie 20 Tage. Entscheidender ist indes, dass mit Art. 37j Abs. 3 E-BankG nunmehr erstmals eine echte Auszahlungsfrist im Gesetz statuiert werden soll. Nachdem der Konkursliquidator die Einleger gemäss Auszahlungsplan umgehend um Zahlungsinstruktionen zur Auszahlung der gesicherten Einlagen ersucht hat (Art. 37j Abs. 2 E-BankG), ist er gehalten, die gesicherten Einlagen spätestens nach 7 Tagen seit Erhalt der Zahlungsinstruktion auszubezahlen. Dies stellt einen Paradigmenwechsel dar, hatten doch bisher keine festen Auszahlungslimiten bestanden. Auch wenn nach wie vor Unsicherheiten in Bezug auf den Zeitraum bestehen, innerhalb dessen der Rücklauf der Zahlungsinstruktionen erwartet werden kann, besteht nun doch erstmals 
eine Auszahlungsfrist. Wenn man den Aspekt der Liquiditätssicherung der Einleger für die Ausgaben des täglichen Bedarfs ernst nimmt, muss es eine verbindliche Frist geben, bis zu welcher diese über das Geld verfügen können.

\section{b) Vorbereitungsarbeiten der Banken}

Die Verkürzung der Auszahlungsfrist ist nur realistisch, wenn die Banken entsprechende Vorarbeiten leisten. Aus diesem Grund sieht Art. 37h Abs. 3 Bst. d E-BankG als Grundprinzip vor, dass jede Bank verpflichtet ist, im Rahmen der ordentlichen Geschäftstätigkeit die notwendigen Vorkehrungen zu treffen, damit der Konkursliquidator den Auszahlungsplan erstellen, die Kontaktierung der Einleger vornehmen und die Auszahlung der Einlagen nach Art. 37j E-BankG gewährleisten kann. Im Vordergrund steht das Erstellen der sog. Single Customer View, d.h. die Fähigkeit der Bank, ihre sämtlichen Kundenbeziehungen quasi auf Knopfdruck abzurufen und sich einen Überblick verschaffen zu können. Absatz $3^{\text {bis }}$ der eingangs genannten Bestimmung konkretisiert die gemäss Absatz 3 Bst. d zu leistenden Vorarbeiten. Entscheidend ist das Erstellen einer Einlegerliste (sog. Masterfile), die sämtliche Einleger mit gesicherten Einlagen zum Gegenstand hat. Sie bildet die Grundlage für den Konkursliquidator zur effizienten und raschen Einholung der Zahlungsinstruktionen. ${ }^{38}$. Daneben werden im Gesetz die Pflicht zur Bereitstellung einer angemessenen Infrastruktur und standardisierter Prozesse sowie die Pflicht zur Erstellung einer summarischen Liste der privilegierten, aber nicht gesicherten Einlagen als notwendige Vorarbeiten genannt.

\section{c) Exkurs: Vorbereitungsarbeiten und systemrelevante Banken}

Die Botschaft ${ }^{39}$ spricht bei den systemrelevanten Banken und den von diesen $\mathrm{zu}$ verlangenden Vorbereitungshandlungen von einem Zielkonflikt. Nicht zu verkennen ist, dass diese einen Notfallplan zu erstellen haben und dort den Nachweis zu erbringen haben, dass die systemrelevanten Funktionen, wozu die Einlagen gehören, auch bei drohender oder eintretender Insolvenz ununterbrochen weitergeführt werden können. Der Konkurs einer systemrelevanten Bank, in welchem Fall die Einlagensicherung ausgelöst würde, ist zwar, so ist zu hoffen, unwahrscheinlich, aber nicht unmöglich. Er stellt ein TailRisiko dar, das nicht vernachlässigt werden darf. Selbst wenn der Nachweis der

38 Vgl. BBl 2020, 6406. Der Anspruch besteht darin, Zahlungsinstruktionen der Einleger, sobald sie eintreffen, automatisiert zu verarbeiten.

39 Vgl. BBl 2020, 6406. 
Weiterführung der systemrelevanten Funktionen erstellt ist, wird es im Insolvenzfall eine Schnittstelle zur Einlagensicherung geben. Der Zielkonflikt wird zumindest für den letztgenannten Fall aufgelöst werden können.

In der Botschaft ${ }^{40}$ wird weiter auf praktische Probleme im Zusammenhang mit der Kontaktierung der grossen Anzahl Kunden der systemrelevanten Banken und der Verarbeitung der Zahlungsinstruktionen (die Anzahl Kunden gehen in die Millionen) hingewiesen. Zuzugeben ist, dass diesbezüglich praktische Herausforderungen bestehen. Auch möglichen Überschneidungen mit den Notfallplänen ist angemessen Rechnung zu tragen. Zusammen mit den Betroffenen müssen Wege gesucht werden, um die Implementierung der Pflichten gemäss Art. 37h Abs. 3 Bst. d und Abs. ${ }^{\text {bis }}$ E-BankG bei den systemrelevanten Banken unter konsequenter Beachtung des Verhältnismässigkeitsprinzips anzugehen. Auf der anderen Seite gelten die erwähnen gesetzlichen Bestimmungen für sämtliche Banken, demnach auch für die systemrelevanten.

\section{d) Finanzierung}

Seit längerem wird die Forderung nach mind. einer teilweisen ex-ante Finanzierung der Beitragsverpflichtungen der Banken erhoben. Die Vorteile der exante Finanzierung liegen auf der Hand. Das Erheben von Beiträgen im Anwendungsfall, namentlich wenn in einer Systemkrise mehrere Banken betroffen sind, wirkt prozyklisch. Ferner leisten auch diejenigen Institute, die vom Konkurs betroffen sind, ihren Beitrag im Rahmen der vorab zu erfüllenden Leistung. Es ist daher zu begrüssen, dass Art. 37h Abs. 3 Bst. c vorsieht, dass im Umfang der Hälfte der Beitragspflicht und auf Dauer entweder leicht verwertbare Wertschriften von hoher Qualität oder Schweizer Franken in bar bei einer sicheren Drittverwahrungsstelle zu hinterlegen sind oder esisuisse ein Bardarlehen zu gewähren ist. Es entfällt in diesem Umfang die Pflicht zur Vorhaltung von Liquidität im Sinne von Art. 37h Abs. 3 Bst. c BankG. In Bezug auf die Anforderungen an die Hinterlegung (Qualität der Wertschriften, Geeignetheit der Drittverwahrungsstelle etc.). und den Mehrwert der Hinterlegung ist auf die Botschaft zu verweisen. ${ }^{41}$

Auch die Obergrenze der gesamten Beitragsverpflichtungen wird einer leichten Anpassung unterzogen. Da die gesicherten Einlagen seit der Einführung der 6-Milliarden-Obergrenze angewachsen sind, war eine Anhebung geboten, um das ursprünglich Schutzniveau wiederum gewährleisten zu können. Die Bezugsgrösse zur Berechnung der Obergrenze soll auf neu 1.6\% der gesamten

$40 \quad$ BBl 2020, 6406.

$41 \quad$ BBl 2020, 6380 f. und 6406. 
gesicherten Einlagen zu stehen kommen (Art. 37h Abs. 3 Bst. b E-BankG). Derzeit betragen die $1.6 \%$ ca. CHF 7.3 Milliarden. Um den Schwankungen der Gesamtsumme der gesicherten Einlagen Rechnung zu tragen, wurde eine Untergrenze bei der geltenden Obergrenze von CHF 6 Milliarden festgelegt.

\section{Bewertung der aktuellen Revision}

Die Revision des Einlagensicherungssystems ist ein Schritt in die richtige Richtung. Es sind Vorteile im Bereich der Auszahlungsfristen, der ex-ante Beitragsfinanzierung sowie der Pflicht der Banken, sich auf das Eintreten des Auszahlungsfalles im Rahmen des ordentlichen Geschäftsganges vorzubereiten, auszumachen. Dem stehen Schwächen des Systems gegenüber, die auch diesmal kein Gehör gefunden haben. Siehe auch in der Botschaft Seiten 23ff., wo dargestellt wird, welche Massnahmen geprüft, aber verworfen wurden. ${ }^{42}$ Nach wie vor ist nur ein Teil der Beitragsverpflichtungen vorfinanziert, was die Ansteckungsgefahr unter den Banken im Rahmen der Erfüllung der Leistungspflicht erhöht. Sodann ist es zwar sachgerecht, eine Obergrenze für die Beitragsverpflichtungen der Banken vorzusehen. Wenn diese aber nicht ausreicht, z.B. in einer Systemkrise, müsste das Gesetz eine Möglichkeit vorsehen, wie die Finanzierungslücke geschlossen werden kann. ${ }^{43}$ Für ein glaubwürdiges Einlagensicherungssystem wäre ein irgendwie geartetes Auffangnetz bei nicht ausreichenden Beitragsverpflichtungen sehr wichtig. Diesbezüglich ist im Rahmen der vorliegenden Revision eine Chance ausgelassen worden.

\section{Angrenzende Gebiete}

\section{Segregierung}

a) Begriff

Unter Segregierung versteht man die Pflicht zum getrennten Halten von Eigen- und Kundenbeständen kontoverbuchter Vermögenswerte (Bucheffekten). Neu erfasst von der Segregierungspflicht werden die gesamten Verwah-

42 Siehe dazu auch die Technical Note des IWF zum Krisenmanagement im Rahmen des 2019 in der Schweiz durchgeführten Financial Sector Assessment Programms (FSAP), publiziert auf der Internetseite des IWF.

43 Siehe zu den verschiedenen Möglichkeiten BBl 2020, 6382. Die Begründung, wonach der Zugriff beispielsweise auf staatliche Fazilitäten zu Fehlanreizen („moral hazard“) führt, ist nicht schlüssig und wird auch nicht begründet. 
rungsketten im Inland und die erste Verwahrungsstelle im Ausland. Die Erfassung dieser Tatbestände macht eine Revision des Bucheffektengesetzes (BEG) erforderlich.

\section{b) Zweck und Inhalt der Segregierungspflicht}

Zweck der Revision der BEG ist die Verbesserung des Anlegerschutzes der Inhaber von Wertschriftendepots vor Unterdeckung. Depotwerte stehen anders als Einlagen im Eigentum der Kunden und werden im Bankenkonkurs ausserhalb der Kollokation abgesondert und den Berechtigten herausgegeben (Art. 37d BankG). Um zu verhindern, dass Kundenbestände zur Befriedigung von Ansprüchen der Bank verwendet werden, müssen Kunden- und Eigenbestände getrennt gehalten werden. Da im Bereich der Bucheffekten die Segregierungspflicht derzeit nur für Zentralverwahrer vorgeschrieben ist (Art. 69 FinfraG), aber noch nicht für Drittverwahrer, gilt es die Lücke zu schliessen.

Art. 11a E-BEG Segregierung soll daher wie folgt angepasst werden (summarische Darstellung): ${ }^{44}$

- Absatz 1: Verankerung Segregierungspflicht für die Verwahrungsstelle;

- Absatz 2: Eigen- und Drittbestände werden von der Verwahrungsstelle bei einer Drittverwahrungsstelle im Inland gehalten. Pflicht zur Verwahrung auf verschiedenen Effektenkonten;

- Absatz 3: Verankerung Segregierungspflicht mit der ersten ausländischen Drittverwahrungsstelle;

- Absatz 4: Bei Unmöglichkeit der Vereinbarung der getrennten Haltung: Schweizer Verwahrungsstelle muss dem Kontoinhaber vergleichbaren Schutz bieten;

- Absatz 5: Ausnahmen zu Absatz 4; und

- Absatz 6: Informationspflichten der Schweizer Verwahrungsstelle: vorgängige standardisierte Kundeninformation. Spezifizierung der Informationspflichten in Bst. a - d (Risikoaufklärung, Kosten der Verwahrung).

\section{c) Datenübermittlung}

Der offen abgefasste Art. 11b Abs. 1 E-BEG bezweckt, den mit der Verwahrung im Ausland befassten Stellen die im Zusammenhang mit der ausländischen Verwahrung erforderlichen Daten möglichst weitreichend zur Verfügung zu stellen. Eine individuelle Entbindung vom Bankkundengeheimnis ist nicht mehr notwendig. Immerhin soll die Einschränkung gelten, dass nur Daten

\footnotetext{
44 Ausführlich BBl 2020, 6416.
} 
offengelegt werden, die der ausländische Verwahrer benötigt, um seinerseits seine (Informations-)Pflichten zu erfüllen. Laut Botschaft ${ }^{45}$ soll die Datenübermittlung die ausländischen Behörden bei der Bekämpfung der Geldwäscherei unterstützen. Absatz 2 des genannten Artikels sieht Informationspflichten der Verwahrungsstelle in ähnlicher Weise wie Art. 11 Abs. 6 E-BEW vor.

\section{Revision Pfandbriefgesetz}

Der Vollständigkeit halber sei auf die Revision des Pfandbriefgesetzes hingewiesen. Es wird Art. 40 PfG revidiert und ein neuer Art. 40a PfG vorgesehen.

Bei Art. 40 PfG soll das System proaktiv geschützt werden können, wenn eine Pfandbriefzentrale oder ein Mitglied, das der Zentrale Darlehen schuldet, gesetzliche Vorschriften, wie z.B. Eigenmittelvorschriften, verletzt oder das Vertrauen ernsthaft beeinträchtigt ist. Die FINMA kann diesfalls für Ordnung sorgen, indem sie einen Untersuchungsbeauftragten einsetzt oder die Aushändigung der Deckungswerte anordnet.

Art. 40a PfG hat ebenfalls den Systemschutz zum Zweck, richtet sich aber ausschliesslich gegen in Konkurs geratene Mitglieder. Dadurch kann das Pfandbriefsystem ernst gefährdet werden. Die Darlehen werden mit der Eröffnung des Konkurses über das Mitglied nicht fällig und die FINMA ordnet deren Separierung einschliesslich der Deckungen an (Abs. 1). Die FINMA setzt weiter einen Beauftragten mit der Aufgabe der Verwaltung der Darlehen ein, um die vollständige und fristgerechte Erfüllung der Pflichten der Darlehensnehmer zu gewährleisten (Abs 2). Falls erforderlich, können die Darlehen samt Deckung mit Genehmigung der FINMA übertragen werden (Abs. 3).

\section{Schlussbetrachtungen}

Die Vorlage kann insgesamt als gelungen bezeichnet werden. Die dringend erforderlichen Arrondierungen im Bereich des Bankeninsolvenzrechts werden weitgehend vorgenommen. Im Bereich der Einlagensicherung sind erste positive Schritte im Hinblick auf die Verbesserung der Wirksamkeit und Funktionsfähigkeit des Systems eingeleitet worden. Es bleibt aber abzuwarten, wie sich die Mitwirkungspflichten der Banken zur Gewährleistung der verkürzten Auszahlungsfrist in der Praxis bewähren/umgesetzt werden. In diesem Zusammenhang ist auf die fünfjährige Übergangsfrist zur Implementierung dieser Pflichten hinzuweisen, die nach der hier vertretenen Auffassung am oberen Ende liegen. Von einer angemessenen Verkürzung der Übergansfrist, 
z.B. auf 2 oder 3 Jahre, würde die Funktionsfähigkeit der Einlagensicherung profitieren. Schliesslich ist die Einführung der Segregierungspflicht positiv zu werten, kann doch die Schweiz als massgebender internationaler Vermögensverwaltungsplatz eine wichtige Lücke schliessen und die Einhaltung entsprechender internationaler Vorgaben unter Beweis stellen. 



\title{
Zuletzt erschienene Bände bei EIZ Publishing, Zürich
}

\author{
Band 201 Challenges, risks and threats for security in Europe \\ 11th Network Europe Conference, Warsaw, 19th-22nd May 2019 \\ ANDREAS KELLERHALS/TOBiAS BAUMGARTNER (Hrsg.), mit Beiträgen von Viorel \\ Cibotaru, Attila Vincze, Przemyslaw Saganek, Jelena Ceranic, Aleksei V. \\ Dolzhikov, Alena F. Douhan, Darina Dvornichenko, Vadym Barskyy, Itay \\ Fischhendler, Verena Murschetz, Jürgen Scheffran, Tobias Baumgartner, 2019 - \\ CHF 49.90/39.90. \\ Band $202 \quad$ Elftes Zürcher Präventionsforum \\ Neue Technologien im Dienste der Prävention: \\ Möglichkeiten - Risiken \\ Christian SChWARZENEGGER/Rolf NäGELI (Hrsg.), mit Beiträgen von Ulf Blanke, \\ Ladina Cavelti, Ulrich Schimpel, Jasmine Stössel, Thomas Wenk, Bettina Zahnd, \\ 2020 - CHF 49.90/39.90.
}

Band 203 Jahrbuch Wirtschaftsrecht Schweiz - EU

Überblick und Kommentar 2019/2020

ANDREAS KELLERHALS/TOBIAS BAUMGARTNER (Hrsg.), mit Beiträgen von Tobias Baumgartner, Mathis Berger, Alexander Brunner, Theodor Bühler, Balthasar Dengler, Jana Fischer, Alfred Früh, Thomas Geiser, Pascal Grolimund, Stefan Härtner, Ulrike I. Heinrich, Isabel Höhener, Samuel Jost, Brigitta Kratz, David Mamane, Laura Manz, Michael Mayer, Urs Meier, Peter Rechsteiner, Antoine Schnegg, René Schreiber, Kurt Sieht, Stefan Sulzer, Wesselina Uebe, 2020 CHF 49.90.

Band $204 \quad$ Kapitalmarkt - Recht und Transaktionen XV

THOMAS U. REUTTER/THOMAS WERLEN (Hrsg.), mit Beiträgen von Marion Bähler, Christina Del Vecchio, Olivier Favre, Jürg Frick, Arie Gerszt, Sonja Maire, Alex Nikitine, Thomas U. Reutter, Annette Weber, 2020 - CHF 39.90.

Band 205 Verantwortlichkeit im Unternehmensrecht X Verantwortlichkeitsprozesse - Tagungsband 2020 Rolf Sethe/Peter R. Isler (Hrsg.), mit Beiträgen von Lukas Fahrländer, Peter Forstmoser, Peter R. Isler, Marcel Küchler, Stephan Mazan, Peter Reichart, Ernst F. Schmid, Rolf Sethe, Martin Waldburger, 2021 - CHF 44.90.

Band 206 Gewalt gegen Frauen

Fachtagung Bedrohungsmanagement - Tagungsband 2019 Christian SchWARZENEGGER, ReinHARd BRunNer (Hrsg.), mit Beiträgen von Reinhard Brunner, Regina Carstensen, Rosa Maria Martinez, Rahel Ott, Christian Schwarzenegger, Luzia Siegrist, Claudia Wiederkehr, 2021 CHF 39.90 .

Band 207 Venture Capital Reinvented: Markt, Recht, Steuern

7. Tagung zu Private Equity - Tagungsband 2020

Dieter Gericke (Hrsg.), mit Beiträgen von Martin Frey, Dieter Gericke, Reto Heuberger, Margrit Marti, Lukas Morscher, Daniel Oehri, Julia Schieber, Lukas Staub, Oliver Triebold, Christian Wenger, 2021 - CHF 44.90. 


\section{Zuletzt erschienene Monografien bei EIZ Publishing, Zürich}

\section{Schweiz - Europäische Union}

Grundlagen, Bilaterale Abkommen, Autonomer Nachvollzug

Matthias OEsch, 2020 - CHF 44.90.

\section{Ein Plus für die Demokratie}

Minimalstandard für die Mitsprache von Parlament und Volk

beim Rahmenabkommen oder bei weiteren Verträgen mit der EU

THOMAS PFISTERER, 2021 - CHF 44.90/34.90.

Internet Governance at the Point of No Return

ROLF H. WEBER, 2021 - CHF 39.00.

Grundprobleme der Invaliditätsbemessung in der Invalidenversicherung

Philipp Egli, Martina FilipPo, Thomas GäChter, Michael E. Meier, 2021 - CHF 54.90/44.90. 
Dieser Band versammelt, teilweise in erweiterter Form, die Referate der 11. Tagung "Sanierung und Insolvenz von Unternehmen“, die das Europa Institut an der Universität Zürich im Juni 2020 online durchgeführt hat.

Im Zentrum steht die aktienrechtliche Sanierung. Ein Schwergewicht liegt bei der Buchhaltung. Behandelt wird sodann mit dem Stillhalteabkommen eine wichtige Form aktienrechtlicher Sanierungsmassnahmen. Beleuchtet werden auch die Sanierung nach neuem Aktienrecht sowie die Sanierung und Einlagensicherung nach neuem Bankenrecht.

Mit Beiträgen von:

Dr. Marc Bernheim

Sikander von Bhicknapahari

Gaudenz Geiger

Dr. Oliver Kälin

Livia Keller

Brigitte Knecht

Dr. Giorgio Meier-Mazzucato

Dr. Reto Schiltknecht 